Aus der Poliklinik für Kieferorthopädie

(Prof. Dr. med. dent. D. Kubein-Meesenburg)

im Zentrum Zahn-, Mund- und Kieferheilkunde

der Medizinischen Fakultät der Universität Göttingen

und

aus der Klinik für Unfallchirurgie und Orthopädie,

Plastische- und Wiederherstellungschirurgie

(Prof. Dr. med. K. M. Stürmer)

im Zentrum Chirurgie

der Medizinischen Fakultät der Universität Göttingen

\title{
Abhängigkeit der Segmentkinematik von der Position der Vorlast im Segment L3/L4
}

\author{
INAUGURAL-DISSERTATION \\ zur Erlangung des Doktorgrades \\ der Medizinischen Fakultät der \\ Georg-August-Universität zu Göttingen \\ vorgelegt von \\ Andrea Kim Charlotte Reitt, geb. Lehmann \\ aus \\ Achim
}

Göttingen 2014 
Dekan:

Prof. Dr. rer. nat. H. K. Kroemer

I. Berichterstatter/in: PD Dr. Martin Wachowski

II. Berichterstatter/in: Prof. Dr. Hans Nägerl

III. Berichterstatter/in: Prof. Dr. Margarete Schön

Tag der mündlichen Prüfung: 17.06.2015 


\section{Inhaltsverzeichnis}

Inhaltsverzeichnis................................................................................................... III

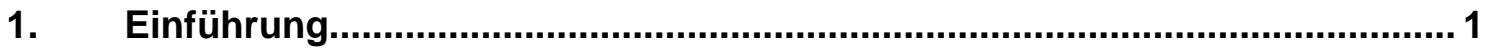

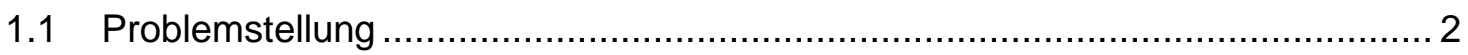

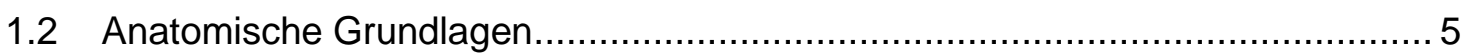

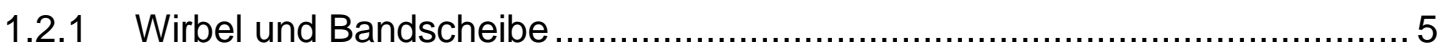

1.2.2 Bänder und Muskulatur....................................................................... 7

1.2.3 Merkmale der Lendenwirbelsäule ...................................................... 8

1.2.4 Synarthrose und Diarthrose ........................................................... 8

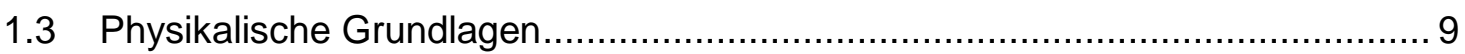

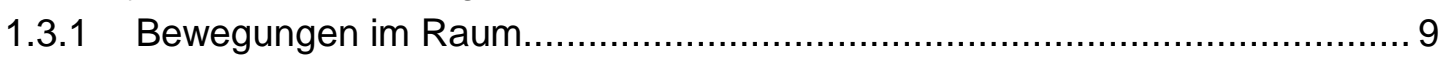

1.3.2 Kraft und Drehmoment................................................................ 10

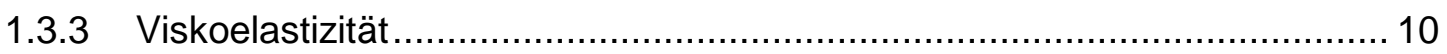

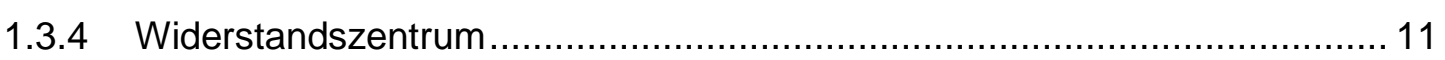

1.3.5 Biomechanische Prinzipen von Diarthrosen............................................ 12

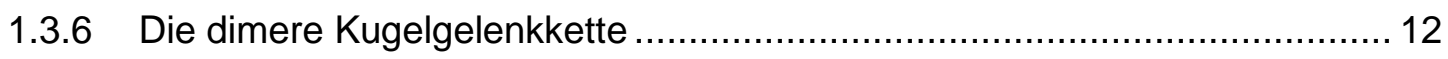

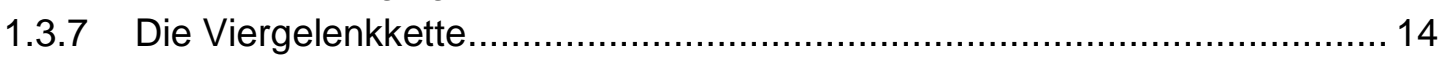

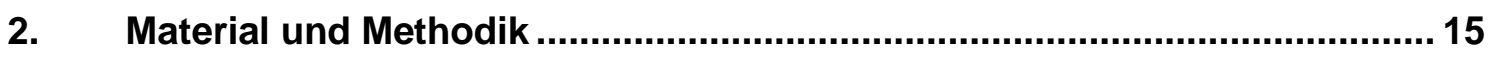

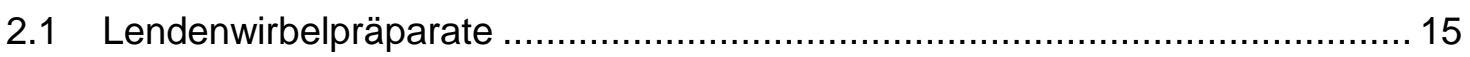

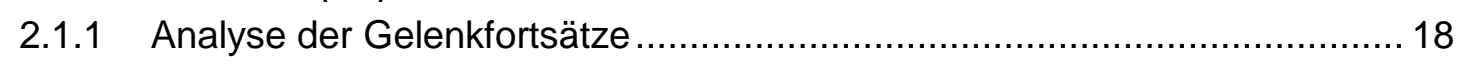

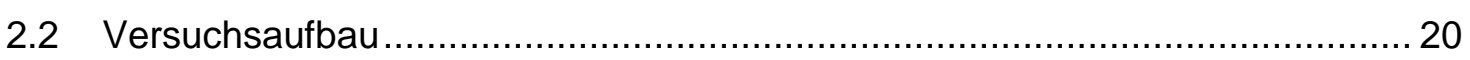

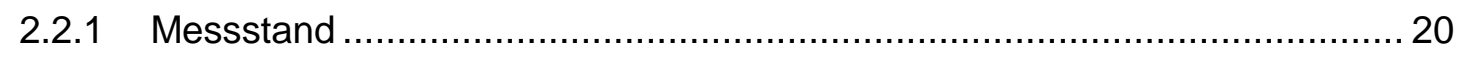

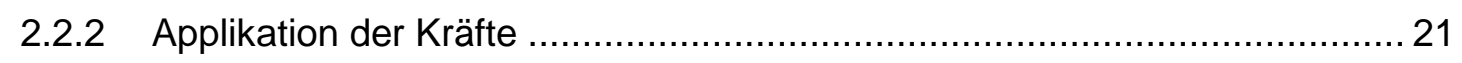

2.2.3 Messtaster, Messinterface und Software ............................................ 24

2.2.4 Präzisionsschraube und Bandscheibenmodelle ....................................... 25

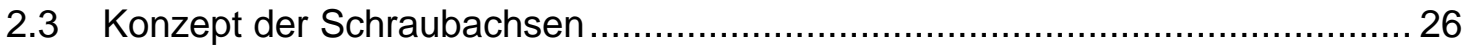

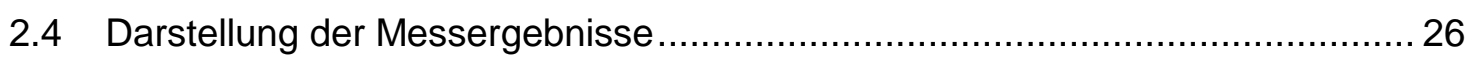

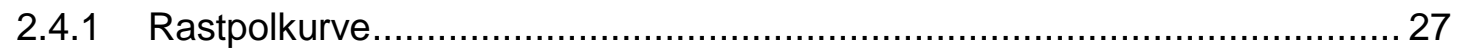

2.4.2 Ausrichtung der momentanen Schraubachsen ...................................... 28

2.4.3 Drehwinkel-Drehmoment-Kennlinie und Bewegungsausmaß ..................... 29

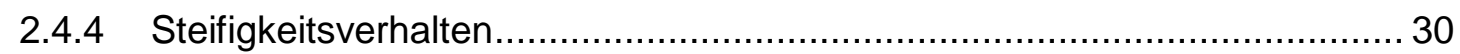

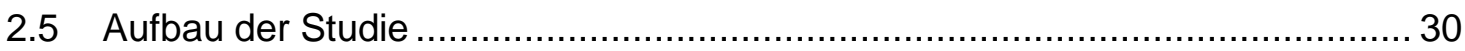

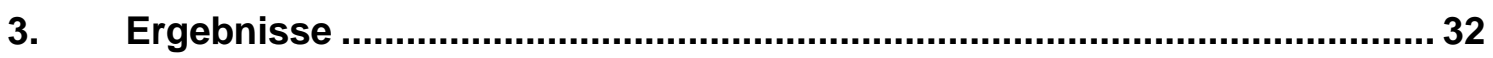

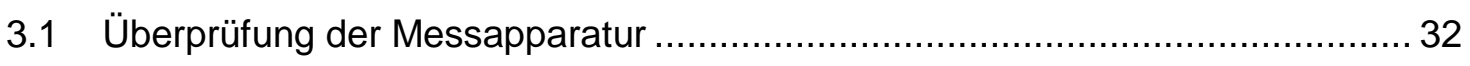

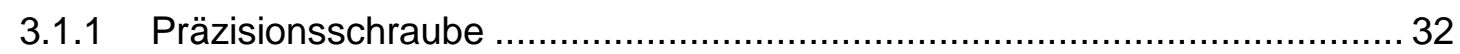

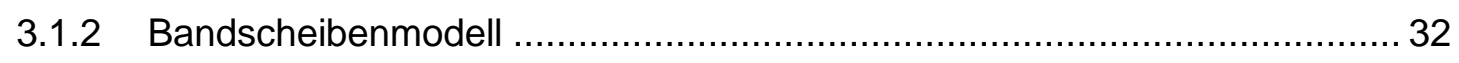

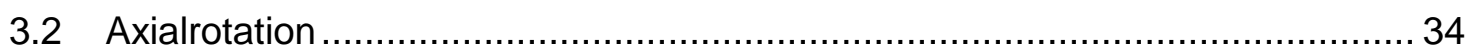

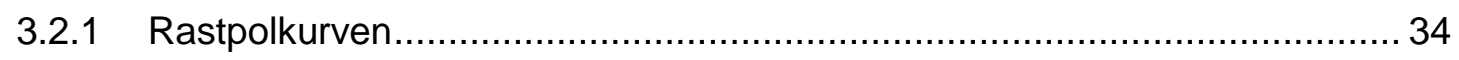




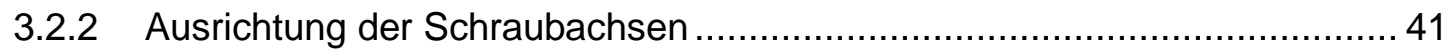

3.2.3 Drehwinkel-Drehmoment-Kennlinie und Bewegungsausmaß ................... 47

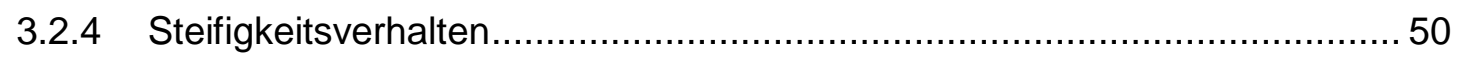

3.2.5 Zusammenfassung der Ergebnisse: „Axialrotation“................................. 55

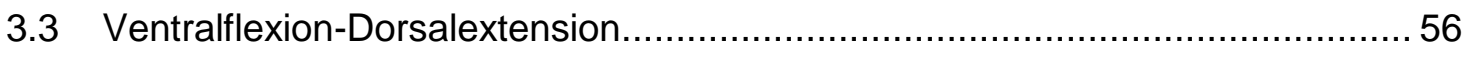

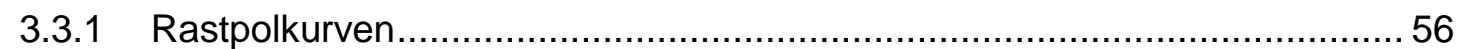

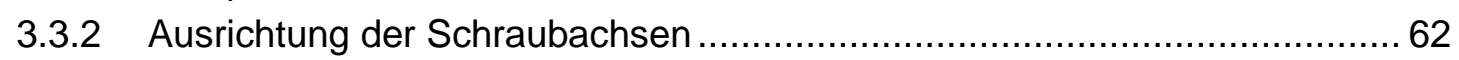

3.3.3 Drehwinkel-Drehmoment-Kennlinie und Bewegungsausmaß .................... 65

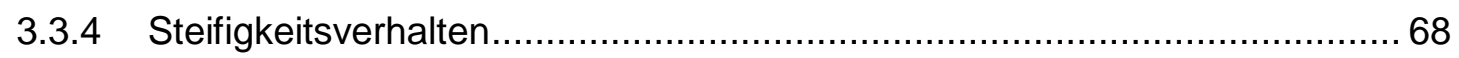

3.3.5 Zusammenfassung der Ergebnisse: „Flexion-Extension“ ........................... 72

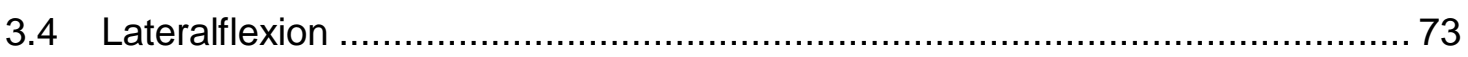

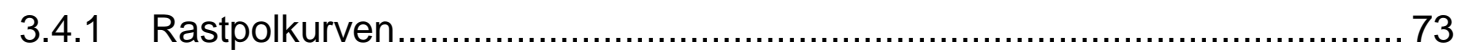

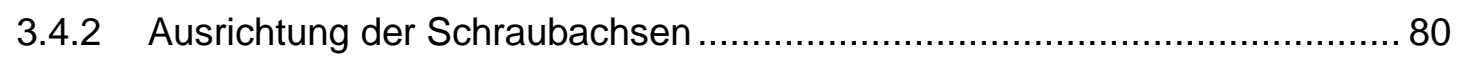

3.4.3 Drehwinkel-Drehmoment-Kennlinie und Bewegungsausmaß ........................ 83

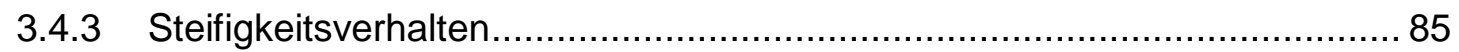

3.4.4 Zusammenfassung der Ergebnisse: „Lateralflexion“ ................................ 88

3.5 Zusammenfassung wichtiger Ergebnisse ........................................... 89

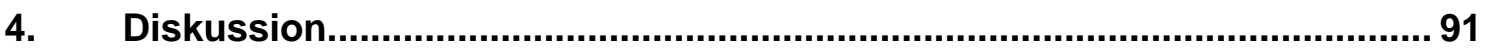

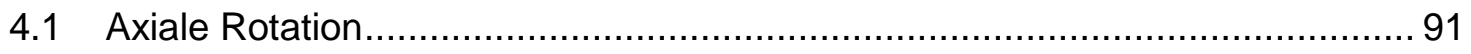

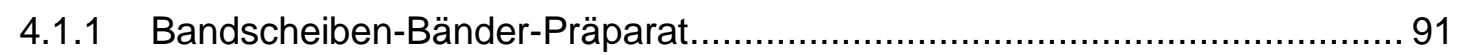

4.1.2 Segment nach Resektion des rechten Zwischenwirbelgelenks .................. 92

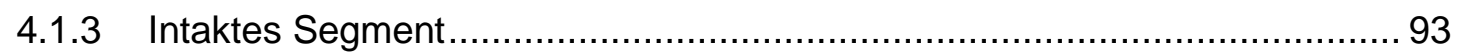

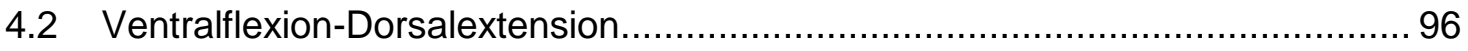

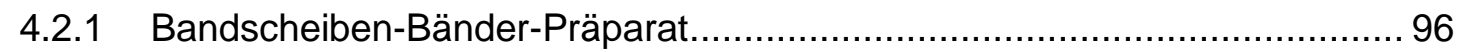

4.2.2 Segment nach Resektion des rechten Zwischenwirbelgelenks .................. 97

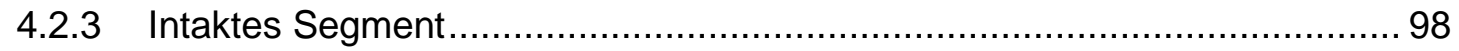

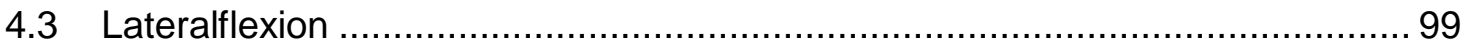

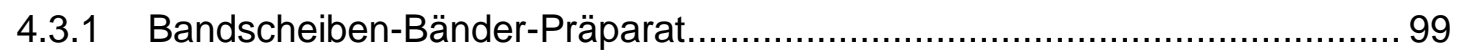

4.3.2 Segment nach Resektion des rechten Zwischenwirbelgelenks ................... 99

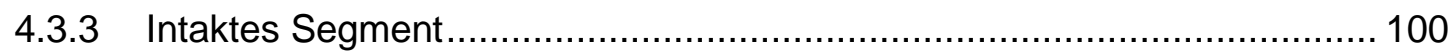

4.4 Singuläre Steifigkeitszunahme als Besonderheit des Segments L3/L4 ......... 102

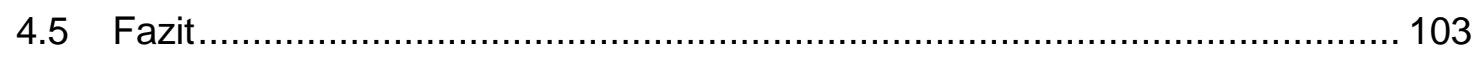

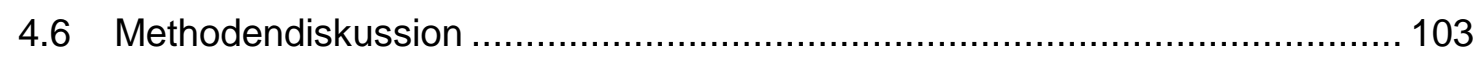

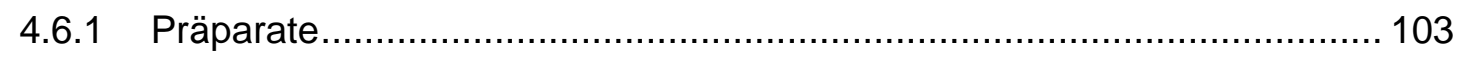

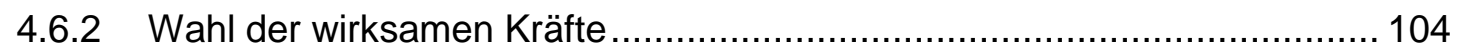

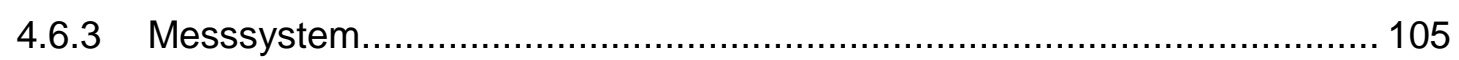

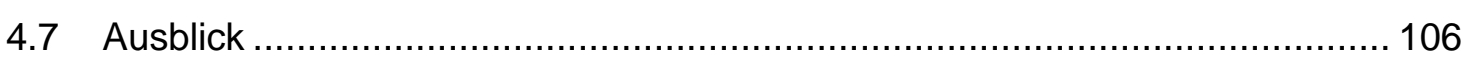

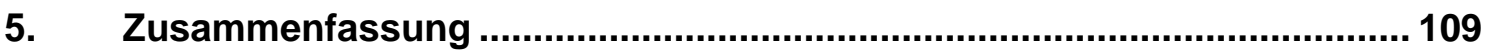

Anhang A: Fehlerabschätzung bei der Erzeugung des Drehmoments................111

Anhang B: Bestimmung der Positionsänderung ................................................113

Anhang C: Berechnung der Schraubparameter ................................................115 


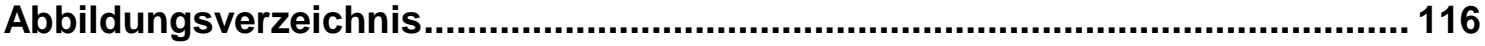

Tabellenverzeichnis.................................................................................................. 119

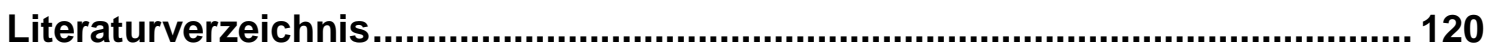




\section{Einführung}

Rückenschmerz ist ein Symptom, dem in der heutigen Gesellschaft eine große Bedeutung beigemessen wird. Der hohe Stellenwert ergibt sich dabei durch steigende Prävalenzen, z.T. starken Leidensdruck, Chronifizierungsrisiko und immense Kosten im Gesundheitswesen. Die zahlreichen Studien, die zu diesem Problem veröffentlicht wurden, zeigen die Wichtigkeit dieser Thematik auf. Im Jahr 2005 gaben 85,5 \% der von Kohlmann und Schmidt befragten Probanden an, in ihrem Leben mindestens einmal an Rückenschmerzen gelitten zu haben. Die Jahresprävalenz lag 2009 in Deutschland bei $60 \%$ (Wenig et al. 2009). Zwar geht man davon aus, dass etwa $90 \%$ der Betroffenen eine spontane Heilung ohne jegliche therapeutische Maßnahmen erfährt, die Rezidivraten liegen jedoch bei ca. $19 \%$ im ersten Jahr nach akutem Schmerzsyndrom und sogar bei 70 \% im weiteren Leben (Watson et al. 1998). Das Auftreten von Rückenschmerzen ist dabei keineswegs ausschließlich ein Symptom der älteren Bevölkerung. Bereits Kinder und Jugendliche im Alter von 10-14 Jahren bzw. 15-18 Jahren litten in einem Zeitraum von neun Monaten zu $21 \%$ bzw. 38 \% unter unspezifischen Schmerzen im unteren Wirbelsäulenbereich (Yao et al. 2011). Die häufigsten Diagnosen bilden dabei mit bis zu $45 \%$ degenerative Veränderungen wie Bandscheibenvorfälle, Spinalkanalstenosen oder Spondylarthrosen (Fanuele et al. 2000, Hart et al. 1995).

Aufgrund der Häufigkeit stellen Beschwerden im Wirbelsäulenbereich gerade unter dem sozioökonomischen Gesichtspunkt eine erhebliche Belastung dar. Die Ausgaben liegen für die sozialen Versicherungssysteme in Deutschland bei jährlich etwa 48 Milliarden Euro. Dabei entstehen 46 \% durch direkte Kosten wie Arztbesuche und Behandlungen, $54 \%$ durch indirekte Kosten durch Arbeitsausfälle. Eine dauerhafte Erwerbsunfähigkeit ist für ca. $17 \%$ der Kosten verantwortlich (Wenig et al. 2009). Unter anderem wird die sehr hohe Chronifizierungsrate als Problem für Rückenschmerzpatienten angegeben. So werden diesbezügliche Beschwerden als zweithäufigster Grund für die Beantragung einer Rente angegeben. In einer australischen Studie wurde ermittelt, dass nach einer sechswöchigen Schmerzepisode nur etwa $50 \%$ der Patienten wieder in ihrem Beruf arbeiteten, nach 13 Wochen mangelhafter Schmerzlinderung lediglich $16 \%$. Zwölf Monate Rückenschmerzen senken die Zahl der Rückkehrer an ihren Arbeitsplatz auf $2 \%$ (Morris und Watson 2011).

Bei solch z.T. langen Krankheitsverläufen ist es nachvollziehbar, dass therapeutisch unterschiedliche Konzepte zur Anwendung kommen. Im ambulanten Rahmen finden sich medikamentöse und lokalanästhetische analgetische Behandlungen zusammen mit therapeutischen Gesprächen im Vordergrund. Weiterhin kommen chiropraktische und physikalische Therapien sowie Krankengymnastik und in selteneren Fällen Akupunktur zur Anwendung. Der Erfolg dieser Verfahren scheint jedoch nur mäßig zu sein. So gibt lediglich ein Drittel der Patienten direkt nach beendeter Therapie eine Besse- 
rung der Schmerzen an. Die Hälfte nimmt keine Veränderung wahr. $18 \%$ berichten sogar von einer Verschlechterung ihrer Symptome (Lang et al. 2000). Dies ist möglicherweise der Grund für die zunehmende Verlagerung auf stationäre Behandlungsmethoden. So ist z.B. im Zeitraum von 2005 bis 2009 die Zahl der operativen Therapieverfahren um $87 \%$ gestiegen (Niethard 2011). Allerdings wurden auch in diesem Konzept noch keine optimalen Lösungen zur Behandlung von Rückenschmerzen gefunden. Ein großes Problem, vor dem die Wirbelsäulenchirurgie steht, ist das postoperative chronische Schmerzsyndrom, das nach Dimova und Lautenbacher (2010) 30-70 \% der Patienten entwickeln. Bisher ist jedoch nicht bekannt, warum besonders Eingriffe an der Wirbelsäule zur Schmerzchronifizierung führen. Allein die hohe Inzidenz dieser postoperativen Komplikation lässt jedoch die Vermutung zu, dass die Kenntnis über das Funktionsprinzip der Wirbelsäule bislang unzureichend erforscht wurde und zudem im klinischen Alltag zu wenig Beachtung findet.

\subsection{Problemstellung}

Das Outcome nach operativer Behandlung von Erkrankungen der Wirbelsäule ist nicht zuletzt abhängig von der Biomechanik im operierten Bereich (Bartels et al. 2008). Bisher wird dem Erhalt der kinematischen Vorgänge im Segment jedoch eine untergeordnete Rolle zugewiesen. Dies mag daran liegen, dass die physiologischen Bewegungsmuster in der Vergangenheit zu selten in den Fokus der Wissenschaft gerückt sind und ihre Relevanz sowohl für ursächliche als auch therapeutische Überlegungen deutlich unterschätzt wurde. Statt des Erlangens eines möglichst physiologischen Zustands steht also vielmehr die kurzfristige Schmerzreduktion im Vordergrund. So wird zur Therapie degenerativer Veränderungen beispielsweise häufig eine Fusion, also eine Versteifung, der betroffenen Wirbelsäulensegmente vorgenommen, was die Ausschaltung physiologischer Bewegungsmuster im betroffenen Wirbelsäulenabschnitt bedeutet. Die Fusion zweier Wirbelsäulensegmente verändert demnach die Biokinematik und -mechanik in der gesamten Segmentkette, woraus langfristig ein verfrühter Verschleiß der angrenzenden Wirbelsegmente resultiert (Hilibrand et al. 1999, Chow et al. 1996, Nielsen et al. 1997, Biederer et al. 1999, Rohlmann et al. 2000). In dem Versuch, diese Langzeitfolgen zu umgehen, werden in den letzten Jahren zunehmend dynamische Bandscheibenprothesen oder interspinöse Implantate eingesetzt. Dauerhafte Erfolge können aufgrund mangelnder Langzeitergebnisse jedoch noch nicht eindeutig nachgewiesen werden (Freeman und Davenport 2006) und möglicherweise nur erzielt werden, wenn durch diese Implantate die physiologische Wirbelsäulenfunktion weitgehend erhalten bleibt. Umso erstaunlicher ist, dass weder die Biomechanik und -kinematik der physiologischen Wirbelsäule noch deren Veränderung nach Einsatz der Implantate bekannt sind. Es fehlen in der Regel selbst bei In-Vitro-Experimenten valide Messungen zu segmentalen Bewegungsvorgängen. Somit offenbart sich für diese Fragestellungen ein erheblicher Nachholbedarf wissenschaftlicher Forschung.

Grundlage für das Verständnis der mechanischen Abläufe in den einzelnen Wirbelsäulensegmenten ist zunächst die Kenntnis über die anatomischen Gegebenheiten und 
die Funktionsprinzipien der an den Bewegungen beteiligten Gelenke. In vielen gängigen Lehrbüchern der Anatomie und Chirurgie wird auch heute noch von einer vereinfachten Darstellung der Funktionsweise der Facettengelenke der Wirbelsäule ausgegangen (Moll und Moll 2006, Benninghoff und Drenckhahn 2008): Sie sei mit formschlüssigen technischen Gelenken gleichzusetzen. Durch einige Arbeitsgruppen (Wachowski et al. 2009, Wetz und Jakob 2001, Nägerl 1990, Nägerl et al. 1992) konnte jedoch aufgezeigt werden, dass diese Annahme sowohl für die Facettengelenke als auch andere Gelenke des Menschen (z.B. Knie) unzureichend ist. Vielmehr führt die Inkongruenz der Gelenkfacetten zu komplexen Relativbewegungen der Wirbelkörper. So ist eine Segmentbewegung mehr als lediglich der Unterschied zwischen Start- und Endzustand, die sich durch eine Winkeldifferenz unterscheiden. Diese Winkeldifferenz beträgt in der Regel nur einige Grad, bei „axialer Rotation“ eines Lendenwirbelsegments nur etwa $\pm 1,5$ Grad (Gregersen und Lucas 1967, Waldeyer und Mayet 1993). Wenn man die Ausgangslage und die Endlage eines Körpers (also des bewegten Wirbelkörpers in einem Segment) kennt, so ist es nach den Gesetzen der räumlichen Kinematik geometrisch immer möglich, den bewegten Wirbelkörper durch eine Schraubung um eine im nicht bewegten Wirbelkörper (Referenz) eines Segments liegende ortsfeste Schraubachse in die Endlage zu überführen. Panjabi et al. (1986) haben eine solche im Referenzsystem ortsfeste Schraubachse (finite helical axis, FHA) aus der gemessenen Anfangslage und der gemessenen Endlage berechnet und diese Schraubung für den tatsächlich stattgefundenen Bewegungsvorgang gehalten. Obwohl sich in dieser Messung die Endlage von der Anfangslage nur um einen Drehwinkel von $\approx 1^{\circ}$ unterschied, ist mit dieser berechneten FHA nicht notwendigerweise der tatsächliche Bewegungsvorgang beschrieben worden, weil das Messsystem von Panjabi et al. nicht erlaubte, Zwischenlagen aufzulösen. Man hätte vielmehr die Auflösung des Messsystems so weit verbessern müssen, dass Zwischenpositionen, die sich durch Drehwinkelunterschiede $\Delta \alpha \ll 1^{\circ}$ unterschieden, auch gemessen worden wären. Damit wäre es möglich gewesen, den kompletten Bewegungsvorgang in annähernd differentiell kleine Schritte aufzulösen, so dass zu jedem der kleinen Intervalle die Lage einer FHA hätte berechnet werden können, um sich den Lagen der momentanen Schraubachse (instantaneous helical axis, IHA) anzunähern. Denn es gilt: IHA $=\lim _{\alpha \rightarrow 0} F H A$. Daher wird in neueren Experimenten das Prinzip der IHA aufgegriffen, wozu die Erfassung von differenziell kleinen Teilbewegungen notwendig ist. Nägerl et al. (1995) haben als Erste eine zur IHA valide Messung zu einem L3/L4- und einem L4/L5- Segment durchgeführt. Später haben auch Bogduk und Mercer (2000) Messungen zur IHA versucht.

Es gibt diverse Herangehensweisen, um das Funktionsprinzip der menschlichen Wirbelsäule wissenschaftlich zu klären. Im Finite-Element-Modell wird auf der Grundlage von anatomischen Daten computergestützt ein Wirbelsäulenmodell erstellt und die Bewegungsabläufe simuliert (Clausen et al. 1997, Wilke et al. 2002, Schmidt et al. 2009). Zwar können mit dieser Methode Parameter separat modifiziert und reproduzierbare Ergebnisse erzielt werden, dennoch liegen bezüglich der Oberflächengeometrie der Gelenkfacetten und der Kinematik bislang noch unzureichende wissenschaftliche Da- 
ten vor. Daher werden die Wirbelbogengelenke massiv simplifiziert in die Modelle eingearbeitet ohne die Geometrie korrekt abzubilden (Schmidt 2008, Dreischarf 2013). Aus diesem Grund sind diese Modelle unzureichend. Einen anderen Ansatz bieten die In-Vivo-Untersuchungen von Baumgartner (2000) und Steffen et al. (1997). Hier wird ein guter Überblick über die biomechanischen Parameter der Wirbelsäule in toto gegeben. Allerdings lassen diese Studien keine Rückschlüsse auf einzelne Abschnitte der Wirbelsäule zu, weil die Auflösung der Messung zu klein ist. Weiterhin liegt ein Nachteil in der starken interindividuellen Variabilität der anatomischen Voraussetzungen und den mangelhaft kontrollierbaren Versuchsbedingungen.

Unter Berücksichtigung der Vor- und Nachteile der bisherigen Verfahren wurde für die vorliegende Studie ein In-Vitro-Verfahren gewählt, das experimentell die wichtigsten Bewegungsparameter eines einzelnen Bewegungssegments beschreibt. Die verwendete Messapparatur ermöglichte dabei die reproduzierbare Einstellung der Versuchsparameter, wobei die Bedingungen isoliert variiert werden konnten. Zudem erlaubte sie die Messung der aufeinander folgenden Positionen des bewegten Wirbelkörpers, die hinreichend klein waren, so dass eine wandernde IHA aufgenommen werden konnte. Bei dieser Apparatur handelt es sich um eine Weiterentwicklung der Apparaturen, die Nägerl et al. (1995) und Mansour (2001) verwendet haben.

Die Kinematik eines jeden Segments ist einzigartig (Wachowski et al. 2009). Um die komplexe Mechanik zu erfassen, werden zumeist Untersuchungen einzelner Segmente durchgeführt (z. B. Wachowski et al. 2007 und 2010b, Nägerl et al. 2009, Rousseau et al. 2006, Mansour 2001). Insbesondere konnte Mansour (2001) zeigen, dass das Segment L3/L4 eine Eigenschaft aufweist, die bislang bei keinem anderen Segment festgestellt werden konnte. Unter Belastung des Segments im Spinalkanal konnte bei Axialrotation ein beträchtlicher Anstieg der Rotationssteifigkeit und damit auch Rotationsstabilität nachgewiesen werden. Sollte dieser Effekt ein typisches Merkmal für diesen Wirbelsäulenabschnitt darstellen, wären die Voraussetzungen für einen deutlichen Fortschritt bei der Behandlung von Erkrankungen in diesem Segment geschaffen. Da dieser Effekt bisher nur an zwei Präparaten gezeigt werden konnte, soll in dieser Studie eine Vertiefung der bisherigen Forschungsergebnisse zur Biomechanik der Lendenwirbelsäule mit einer höheren Zahl an Präparaten stattfinden. Zu diesem Zweck werden in der vorliegenden Arbeit die biomechanischen Bewegungseigenschaften des Lendenwirbelsegments L3/L4 mit großer Genauigkeit dargestellt. Da ein Schwerpunkt auf der Funktion und Bedeutung der Zwischenwirbelgelenke sowie dem Zusammenspiel zwischen Gelenken und der Bandscheibe liegt, wird zunächst ein Überblick über die Anatomie der Wirbelsäule und die mathematisch-physikalischen Grundlagen der Wirbelsäulengelenke gegeben. Nach der Präsentation der Ergebnisse werden diese einer kritischen Begutachtung unterzogen und eine Systematik zur Gelenkfunktion aufgestellt. Ebenso findet ein Vergleich der im Rahmen dieser Arbeit experimentell ermittelten Befunde mit denen anderer Lendenwirbelsäulenabschnitte statt sowie eine Diskussion des von Mansour entdeckten Effekts der singulären Steifigkeitserhöhung. 


\subsection{Anatomische Grundlagen}

Die Zusammenfassung der anatomischen Grundlagen folgt Benninghoff und Drenckhahn (2002) und Schünke et al. (2005).

Der Wirbelsäule (Columna vertebralis) des erwachsenen Menschen kommen als Achsenorgan zwei Hauptfunktionen zu. Zum einen soll sie möglichst stabil sein und das Gewicht von Kopf und Rumpf in verschiedenen Körperhaltungen tragen (statische Funktion), zum anderen die Beweglichkeit des Stammes ermöglichen und Stöße abfedern (kinematisch-dynamische Funktion). Um diesen Ansprüchen gerecht zu werden, müssen verschiedene Gewebe und Systeme miteinander agieren.

Zunächst lässt sich die Wirbelsäule in vier Abschnitte gliedern: die aus sieben Wirbeln bestehende Halswirbelsäule (HWS), die Brustwirbelsäule (BWS) mit ihren zwölf Wirbeln, die Lendenwirbelsäule (LWS), die fünf Wirbel umfasst und das Kreuzbein (Os sacrum), dessen fünf Wirbel nach der Geburt zu einem großflächigen Knochen verschmelzen. Zusätzlich besitzt der Mensch drei bis fünf Wirbelrudimente, die das Steißbein (Os coccygis) bilden. Diese Gliederung beruht einerseits auf Unterschieden in der Bauweise der einzelnen Wirbelkörper, andererseits auf den typischen Krümmungen, die die Wirbelsäule in sagittaler Richtung aufweist. So findet sich im Bereich der Halsund Lendenwirbelsäule eine Lordose, während Brustwirbelsäule und Kreuzbein kyphosiert sind. Damit weist die gesamte Wirbelsäule eine doppelte S-Form auf. Schon durch diese Form ist die Wirbelsäule in der Lage, besonders axial gerichtete Stöße abzufangen und die einwirkenden Kräfte weiterzuleiten.

\subsubsection{Wirbel und Bandscheibe}

Jeder Wirbel ist aus den gleichen Elementen aufgebaut. Er umfasst:

- einen Wirbelkörper (Corpus vertebrae)

- einen Wirbelbogen (Arcus vertebrae)

- $\quad$ einen Dornfortsatz (Processus spinosus)

- zwei Querfortsätze (Proccessi transversi)

- vier Gelenkfortsätze (Proccessi articulares)

Wirbelkörper und Wirbelbogen bilden das Wirbelloch (Foramen vertebrale), durch das das Rückenmark hindurchzieht. Da mehrere Wirbelkörper und damit auch Wirbellöcher übereinander liegen, entsteht der Wirbelkanal (Canalis vertebralis), der dem Rückenmark einen beweglichen Schutzmantel bietet. Die Fortsätze dienen den Muskelfasern als Ursprungs- und Ansatzpunkte und sind an den Bewegungen der Wirbelsäule maßgeblich beteiligt (Abbildung 1). 


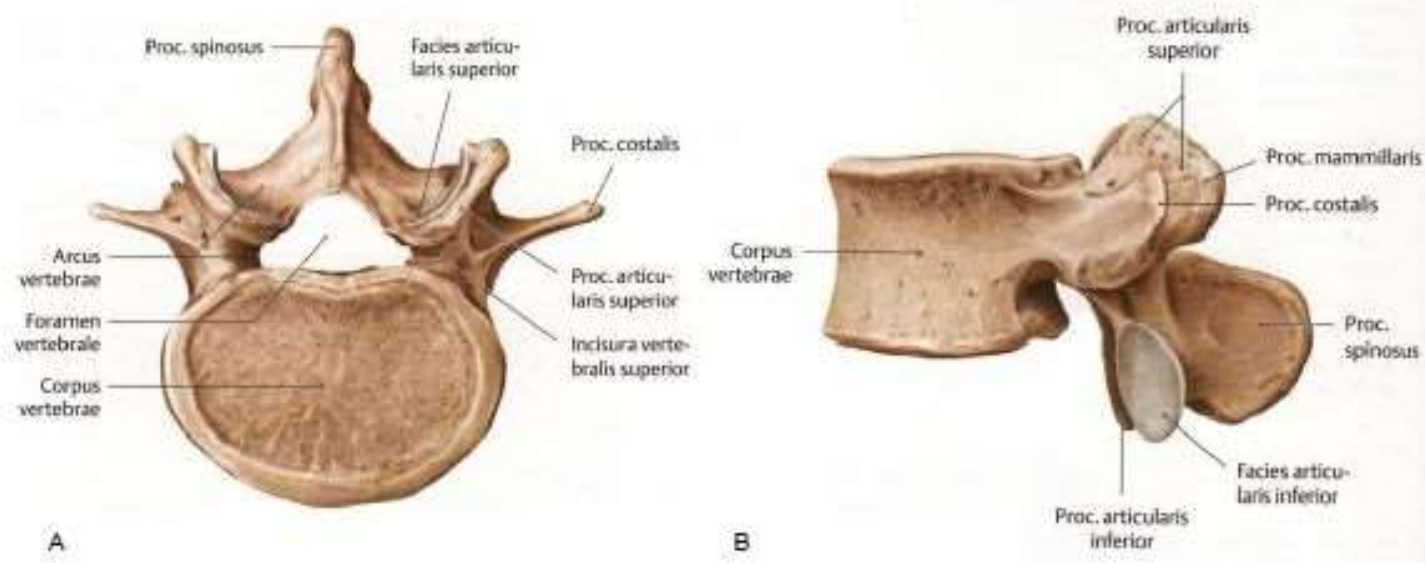

Abbildung 1: Knöcherner LWK 4 von A) kranial und B) seitlich (Schünke et al. 2005, S. 88/89).

Benachbarte Wirbel stehen zum einen durch die Bandscheibe (Discus intervertebralis) in gelenkiger Verbindung, zum anderen über sogenannte Wirbelbogengelenke (Articulationes zygapophysiales). Die Bandscheibe gehört als Gelenk zu den Synarthrosen (s. Abschnitt 1.2.4) und besteht aus einem gallertigen Kern mit hohem Wassergehalt ( $\mathrm{Nu}$ cleus pulposus) sowie einem darum herum ziehenden Faserring (Anulus fibrosus). Die äußeren Fasern verlaufen schräg zwischen den Randleisten der beiden Wirbelkörper. Durch unterschiedliche Steigungswinkel kreuzen sich die Fasern in mehreren Schichten, wodurch die hohe Zugfestigkeit dieses Faserrings zu erklären ist. Zwischen Bandscheibe und Oberfläche des Wirbelkörpers befindet sich eine dünne Knorpelschicht (Deckplatte), die sowohl der Ernährung der Bandscheibe als auch ihrer Verankerung im Wirbelkörper dient.

Neben der Limitierung der Bewegung der Wirbel untereinander kommt der Bandscheibe auch die Funktion eines Stoßdämpfers zu, denn durch den hohen Wasseranteil des Nucleus pulposus lässt sich dieser zwar verformen, nicht jedoch komprimieren. Dadurch wird der durch einen Stoß einwirkende Druck gleichmäßig auf die benachbart liegenden Wirbel bzw. den Anulus fibrosus verteilt und kann von innen abgefangen werden.

Im Zusammenhang damit wurde 1977 von Junghanns der Begriff des Bewegungssegments geprägt. Er umfasst eine Bandscheibe mit den anliegenden Wirbeln und dem zugehörigen Bandapparat (Abbildung 2).

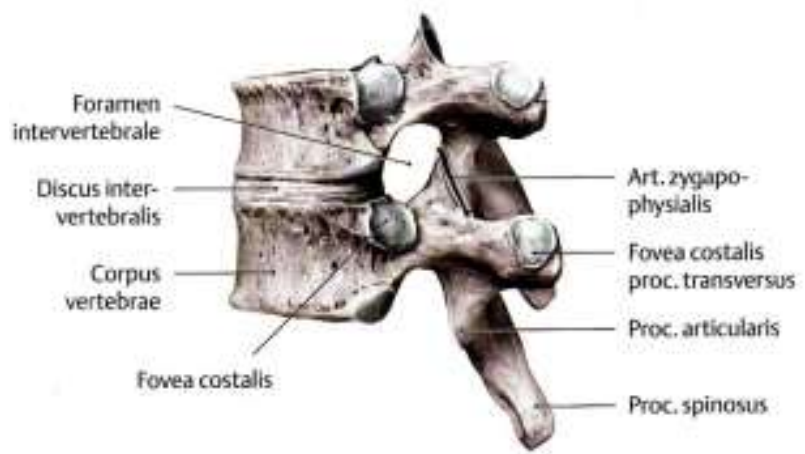

Abbildung 2: Junghans'sches Bewegungssegment, Ansicht von lateral (Schünke et al. 2005, S. 100) 
Bei den paarigen Wirbelbogengelenken handelt es sich um echte Gelenke (Diarthrosen, s. Abschnitt 1.2.4). Dabei artikulieren jeweils ein Gelenkfortsatz des oberen und des unteren Wirbels miteinander. Die Gelenkfacetten bilden die Kontaktstellen der Gelenkfortsätze. Sie sind mit einer glatten Knorpelschicht überzogen. Die Gelenkfacetten des oberen Wirbelkörpers sind konvex geformt, das Gegenstück des unteren Wirbelkörpers konkav. Die Facettenkrümmungen sind dabei inkongruent. Dadurch wird die Variabilität der Artikulationsmöglichkeiten erhöht. Entlang der Wirbelsäule variiert die Stellung der Wirbelbogengelenke zum Wirbelkörper, je nachdem in welchem Wirbelsäulenabschnitt sie liegen. Diese Gelenke sind für die Führung der Bewegungen in der Wirbelsäule zuständig und definieren die Lagen der Achsen, um die Bewegungen erfolgen.

\subsubsection{Bänder und Muskulatur}

Auch Bandstrukturen sorgen für Verbindungen innerhalb der Wirbelsäule und verbessern die Statik durch Aufrechterhaltung einer Grundspannung (Vorspannung). Man unterscheidet Wirbelkörperbänder von Wirbelbogenbändern (Abbildung 3). Zu den Wirbelkörperbändern zählen das Ligamentum longitudinale anterius bzw. posterius, die ventral bzw. dorsal am Wirbelkörper die ganze Wirbelsäule entlang ziehen. Die Wirbelbogenbänder werden von den Ligamenta flava, Ligamenta interspinalia, Ligamenta intertransversaria und dem Ligamentum supraspinale gebildet. Diese verbinden die Wirbelbögen bzw. Dorn- oder Querfortsätze benachbarter Wirbel miteinander. Die Befestigung der Wirbelsäule im knöchernen Becken wird ebenfalls durch Bandstrukturen gewährleistet.

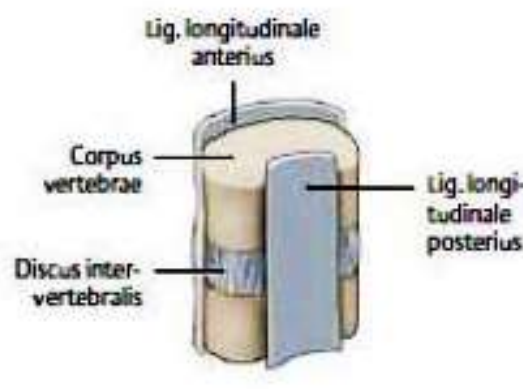

A

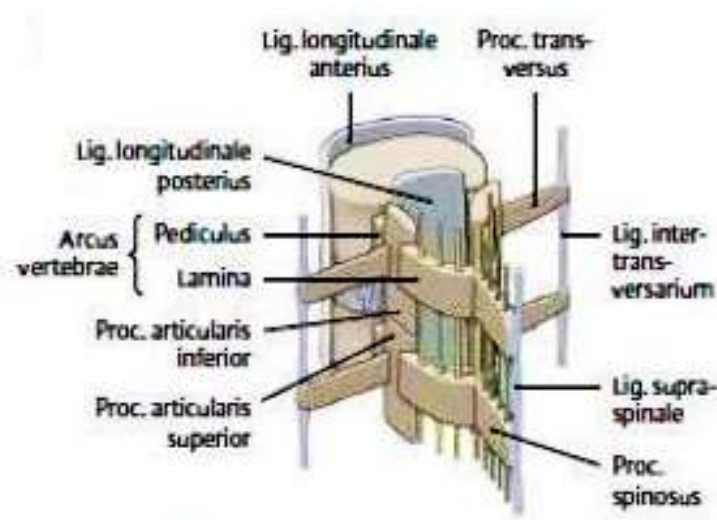

B

Abbildung 3: Schematische Darstellung $\operatorname{der} A$ ) Wirbelköperbänder und $B$ ) Wirbelbogenbänder (Schünke et al. 2005, S. 94)

Durch die komplexe Wirbelsäulen- und Rückenmuskulatur ist eine Vielzahl an mechanisch fein abgestimmten Bewegungen in der Wirbelsäule möglich. Als Hauptbewegungsrichtungen unterscheidet man Axialrotation (Rotation um die Körperachse), Dorsalextension-Ventralflexion (Rück- und Vorbeugung) und Lateralflexion (Seitwärtsbeugung). Die Bewegungen finden jedoch meist nicht isoliert statt, sondern können 
ineinander übergehen oder parallel ablaufen. Weiterhin ist die Haltefunktion der kräftigen Rückenmuskulatur zu erwähnen, die z.B. den aufrechten bipeden Gang ermöglicht.

\subsubsection{Merkmale der Lendenwirbelsäule}

Die Lendenwirbel sind im Vergleich mit den kranial liegenden Wirbeln der HWS und BWS größer und kräftiger ausgebildet. Die Körper sind in der Aufsicht queroval, das Wirbelloch ist nahezu dreieckig. Die Querfortsätze der Lendenwirbel sind eigentlich Rippenrudimente, weshalb sie als Rippenfortsätze (Processi costales) bezeichnet werden. Die Gelenkfortsätze sind in etwa senkrecht ausgerichtet, der gebogene Gelenkspalt verläuft schräg zur Medianebene. Dabei weisen die medial gelegenen unteren Gelenkfortsätze eine konvexe, die oberen eine konkave Form auf.

Im Rahmen des Bewegungsausmaßes der gesamten Lendenwirbelsäule sind besonders Beugungen in der sagittalen Ebene möglich (Ventralflexion $50^{\circ}$, Dorsalextension $35^{\circ}$ ), während Lateralflexion (je $20^{\circ}$ ) und axiale Rotation (je $5^{\circ}$ ) eine untergeordnete Rolle spielen (Schünke et al. 2005).

Der vierte und fünfte Lendenwirbel ist zusammen mit dem Kreuzbein über Bänder fest im Becken verankert. Die Verbindung der beiden Lendenwirbel erfolgt dabei über das Ligamentum iliolumbale, das von den Processi costales zu den Beckenkämmen zieht. Der dritte Lendenwirbel gilt hingegen als letzter „freier Wirbel“, der keine ligamentäre Verbindung zum Becken besitzt.

\subsubsection{Synarthrose und Diarthrose}

Die Bewegungsstruktur von Gelenken folgt je nach Aufbau der gelenkigen Verbindung unterschiedlichen Prinzipien. Man spricht von Synarthrosen, wenn eine durch Füllgewebe vermittelte Verbindung zweier Skelettteile vorliegt, die nicht durch einen Gelenkspalt unterbrochen und von Kapselgewebe umgeben ist (Frick et al. 1992). Als Füllmaterial kommen Binde-, Knochen- oder Knorpelgewebe in Frage. Ein Beispiel für Letztgenanntes stellen die Bandscheiben der Wirbelsäule dar.

Diarthrosen werden dagegen von artikulierenden Gelenkflächen gebildet, die von einem Gelenkspalt unterbrochen sind. Diese sind mit Gelenkknorpel überzogen, um möglichst reibungsfreie Bewegungen zu erlauben. Das Gelenk wird von einer Gelenkkapsel eingefasst (Abbildung 4). 

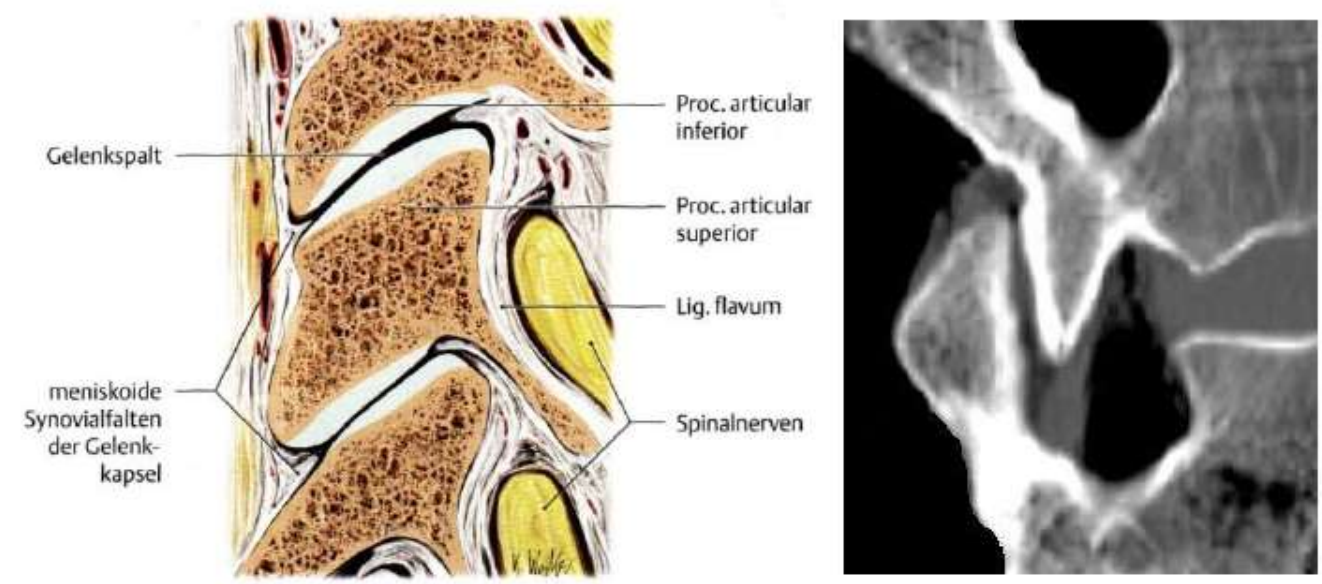

Abbildung 4: Vergrößerte Darstellung von zwei benachbarten Wirbelbogengelenken der HWS (links) (Schünke et al. 2005, S. 100) und CT-Bild eines Wirbelbogengelenks auf Höhe L3/L4 des Versuchssegments C (rechts)

\subsection{Physikalische Grundlagen}

Der folgende Abschnitt soll einen vereinfachten Überblick über die physikalischen Vorgänge geben, die für das Basisverständnis dieser Arbeit wichtig sind. Daher wird an dieser Stelle auf die Darstellung der Aspekte mittels Formeln zur exakten Berechnung weitestgehend verzichtet. Dieser Abschnitt folgt Hering et al. (2007) sowie Klein und Sommerfeld (2007).

\subsubsection{Bewegungen im Raum}

Die Bewegung eines Objekts in einer Ebene kann auf zwei Komponenten zurückgeführt werden: eine Translations- und eine Rotationskomponente (Abbildung 5). Gleiches gilt auch für den dreidimensionalen Raum. Dort addieren sich die Bewegungen in jeder der drei Ebenen zu einer Gesamttranslation bzw. -rotation zusammen. Das Bewegungsvermögen eines Objekts wird in Freiheitsgraden angegeben. Mit diesem Begriff werden die maximal sechs voneinander unabhängigen Bewegungsmöglichkeiten eines Systems bezeichnet. Diese sechs Freiheitsgrade addieren sich also durch drei rotatorische Freiheitsgrade um die drei Raumachsen $x, y$ und $z$ sowie drei translatorische Freiheitsgrade entlang dieser Achsen.

Nach Chasles Satz (Teichmann 1973) handelt es sich bei einer Rotation mit dem Winkel a um eine Achse zusammen mit einer Translation entlang derselben Achse um eine Verschraubung, durch die jede mögliche Bewegung eines Körpers im Raum beschrieben werden kann. Die Schraubsteigung $\zeta$ ergibt sich aus dem Quotienten aus dem Versatz $s$ entlang der Schraubachse und dem Rotationswinkel a um diese Achse: $\zeta=|s| /|\alpha|$. Um die Bewegung eindeutig zu definieren muss neben dem Versatz $s$ sowie dem Drehwinkel a auch der Orts- und Richtungsvektor der Schraubachse bekannt sein. Bei dieser Betrachtung wird allerdings nur von Anfangs- und Endposition und somit von der kürzesten Verbindung zwischen den beiden Positionen ausgegangen. Diese Darstellung mit nur einer Schraubachse, die auch Panjabi et al. (1986) ver- 
wendet haben, erlaubt jedoch keine differenzierte Beschreibung eines Bewegungsvorgangs, denn die zahlreichen möglichen Zwischenpositionen werden außer Acht gelassen. Da aber die Schraubachsen der Teilbewegungen von der Schraubachse der Gesamtbewegung abweichen können (Nägerl et al. 1995), ist es je nach Fragestellung sinnvoll, die Gesamtbewegung in differentiell kleine Lageänderungen zu teilen und daraus Rückschlüsse auf den kompletten Bewegungsablauf zu ziehen.
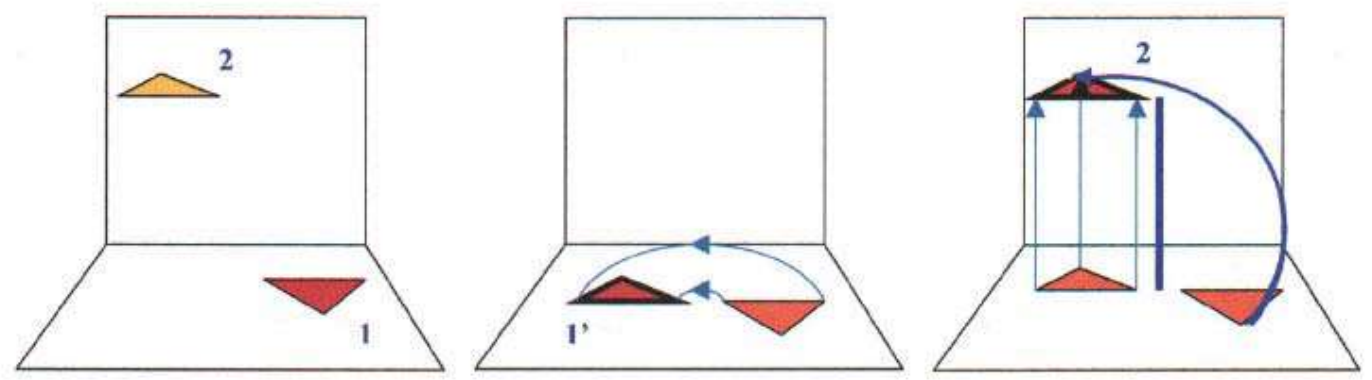

Abbildung 5: Unbekannte Bewegung eines Körpers von 1 nach 2. Die Gesamtbewegung kann in eine Rotation nach 1' und in eine Translation nach 2 aufgeteilt werden (Bockermann 2004, S. 20).

\subsubsection{Kraft und Drehmoment}

Die Kraft $F$ ist eine gerichtete physikalische Größe und wird nach dem zweiten Newton'schen Gesetz durch das Produkt aus Masse $m$ und Beschleunigung a definiert $(F=m * a)$. Die Maßeinheit der Kraft ist Newton N. Sie kann als linienflüchtiger Vektor entlang ihrer Kraftwirkungslinie verschoben werden. Eine Kraft $F$ kann durch eine Gegenkraft -F ausgeglichen werden. Eine Kraft, die im Schwerpunkt eines Körpers angebracht wird, verursacht eine reine Translationsbewegung. Setzt sie hingegen außerhalb des Schwerpunktes an, resultiert ein Drehmoment T. Das Drehmoment wird entsprechend aus dem Kreuzprodukt der Kraft $F$ und dem Hebelarm $L$ (Abstand der Kraftwirkungslinie vom Schwerpunkt) gebildet ( $T=F \times L)$. Per definitionem steht der Vektor des Drehmoments T senkrecht zu F und L. Drehmomente werden in der Einheit Newtonmeter $\mathrm{Nm}$ angegeben. Ein Drehmoment kann als sogenannter freier Vektor im Raum verschoben werden. Ein drehbarer Körper kann durch ein Drehmoment in Bewegung gesetzt werden. Wirkt auf den Körper zusätzlich ein Gegendrehmoment -T $(=-F \times L)$, wird der Körper nicht gedreht.

\subsubsection{Viskoelastizität}

Viskoelastizität beschreibt ein Materialverhalten, das die Eigenschaften von Flüssigkeiten (Viskosität) und Festkörpern (Elastizität) kombiniert. Ein Beispiel für einen Körper mit rein elastischen Eigenschaften ist eine Hook'sche Feder, deren Auslenkung s proportional zur wirkenden Kraft $F$ ist. Kommt nun ein dämpfender visköser Effekt hinzu, verläuft die Bewegung nicht länger reibungsfrei, die Endlage wird verzögert erreicht. Im menschlichen Körper wird dieses viskoelastische Verhalten u.a. von Bändern und Bandscheiben gezeigt. Jeder Richtungswechsel einer Bewegung führt zu einem Dämpfungseffekt, so dass die neue Position nur verzögert erreicht wird. Bei periodisch ange- 
legten sinusförmigen Kräften führt diese Dämpfung zu einer phasenverschobenen Auslenkung, die graphisch als Hysterese dargestellt werden kann.

\subsubsection{Widerstandszentrum}

Jeder freie Körper besitzt einen Punkt, in dem er in Balance gehalten werden kann. Dieser Punkt wird als Massenschwerpunkt bezeichnet. Handelt es sich um einen hinreichend symmetrischen Körper, der über eine elastische Aufhängung verfügt, ist dieser Punkt als Widerstandszentrum bekannt. Betrachtet man ein Wirbelsäulensegment vereinfacht als zwei kreisrunde Wirbelkörper ohne Gelenkfortsätze, die durch eine Bandscheibe verbunden sind (Prinzip einer Synarthrose), so lässt sich das Widerstandszentrum dieses Körpers ermitteln (Nägerl 1990). Dazu wird der Schnittpunkt der vertikalen Symmetrieachse z des Segments mit der Grenzfläche zwischen der Bandscheibe und dem oberen Wirbelkörper bestimmt (Abbildung 6).

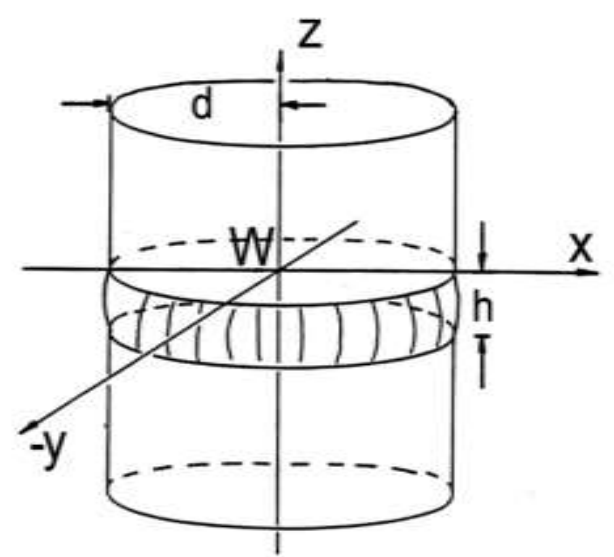

Abbildung 6: Modell einer Synarthrose; Das Widerstandszentrum W liegt in der Symmetrieachse. Die elastische Scheibe hat die Dicke $\mathrm{h}$ und den Durchmesser d. Die Achsen beziehen sich auf das kartesische Koordinatensystem (Mansour 2001, S. 29).

Kräfte, die im Widerstandszentrum an den Körper angreifen, führen zu einer Translationsbewegung in Richtung der angreifenden Kraft. Dies bedeutet, angewandt auf das vereinfachte Wirbelsäulensegment, dass Kräfte, die entlang der $x$ - oder $y$-Achse wirken, zu einer Abscherung des oberen Wirbels führen, während Kräfte entlang der z-Achse in einer Stauchung oder Dehnung der Bandscheibe resultieren. Das Ausmaß der Bewegung ist dabei abhängig von den elastischen Eigenschaften des Körpers, im genannten Beispiel hauptsächlich von denen der Bandscheibe.

Setzen Kräfte außerhalb dieses Punktes an, wird dadurch ein Drehmoment erzeugt und es resultiert eine Rotationsbewegung im Widerstandszentrum. Diese kann, je nach Richtung des Drehmoments, zu einer Neigung des oberen Wirbels oder zu einer Drehung um eine vertikale Achse führen. Für das vorliegende Beispiel einer Synarthrose gilt das Reziprozitätstheorem (Nägerl et al. 1990). Dieses besagt, dass die Rotationsachse einer Bewegung umso näher an das Widerstandszentrum heranrückt, je größer der Abstand zwischen Widerstandszentrum und Kraftwirkungslinie wird und vice versa. 
Das natürliche Wirbelsäulensegment unterscheidet sich hinsichtlich mehrerer Punkte von dem besprochenen vereinfachten Modell. Zum einen ist die Bandscheibe nicht kreisrund sondern, je nach Wirbelsäulenabschnitt, oval und unterliegt in seiner Form individuellen Schwankungen. Weiterhin sind zusätzlich zwei Wirbelbogengelenke an der Bildung eines Segments beteiligt. Aus diesen Gründen ist das Widerstandszentrum in vivo nicht im Zentrum sondern im dorsalen Bereich der Bandscheibe zu erwarten und für jedes Segment eines jeden Individuums separat zu bestimmen.

\subsubsection{Biomechanische Prinzipen von Diarthrosen}

Die Funktion von Diarthrosen setzt voraus, dass die beteiligten Gelenkflächen kraftschlüssig sind. Dies kann durch kompressive Kräfte erreicht werden, die z.B. von Muskeln ausgeübt werden. Da die Gelenkflächen inkongruent sind, kann, je nach Aufbau des Gelenks, eine unterschiedliche Anzahl an Kontaktpunkten der Gelenkflächen hergestellt werden. Eine große Zahl an Kontaktpunkten geht dabei zwar mit einer erhöhten Stabilität einher, dafür sinkt gleichzeitig die Beweglichkeit des Gelenks. Zur Beschreibung des Bewegungsvermögens von Körpern oder Gelenken werden in diesem Zusammenhang Freiheitsgrade verwendet (s. Abschnitt 1.3.1). Ein dimeres Kugelgelenk mit nur einem Kontaktpunkt besitzt nach dieser Systematik fünf Freiheitsgrade. Nach Nägerl (1990) führt jeder zusätzliche Kontaktpunkt zu einem Verlust von einem Freiheitsgrad. Betrachtet man die Zwischenwirbelgelenke eines Wirbelsäulensegments als eine Diarthrose mit zwei möglichen Kontaktpunkten (rechtes und linkes Gelenk), liegt demnach eine gelenkige Verbindung mit vier Freiheitsgraden vor, wenn beide Gelenke kraftschlüssig sind. Verlieren die Gelenkflächen einer Seite den Kontakt, erhöht sich die Zahl der Freiheitsgrade auf fünf. Wird keine kompressive Kraft ausgeübt und sind beide Gelenke daher vollständig entkoppelt, werden sechs Freiheitsgrade erreicht.

\subsubsection{Die dimere Kugelgelenkkette}

Sobald ein Gelenk Kraftschluss erfährt, wird die stattfindende Bewegung durch dieses Gelenk geführt. Ein einfaches Modell, um diese Führung zu erläutern, bietet das Prinzip der „Dimeren Kugelgelenkkette“. Dieses Prinzip kann z.B. auf ein einseitig kraftschlüssiges Zwischenwirbelgelenk eines Wirbelsäulensegments übertragen werden. Bei der dimeren Kugelgelenkkette bestehen die Artikulationsflächen aus einer konvexen und einer konkaven Gelenkfläche. Dabei weisen die kleinere konvexe und die gröBere konkave Einheit einen unterschiedlichen Krümmungsmittelpunkt $\mathrm{M}$ auf (Nägerl et al. 1993a, 1993b, Kubein-Meesenburg et al. 1990). Eine Linie, die die beiden Krümmungsmittelunkte verbindet, wird Kettenglied m genannt. Aus Präsentationsgründen wird im Folgenden die konkave Gelenkfläche als fixierter Körper $\mathrm{G}_{\mathrm{fix}}$ bezeichnet, die konvexe Gelenkfläche als beweglicher Körper $G_{\text {bew }}$ (Abbildung 7). 


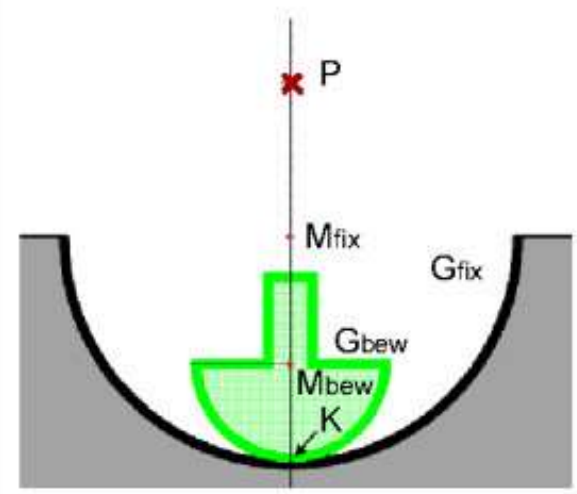

Abbildung 7: Modell einer überschlagenen Gelenkkette. Der bewegte Gelenkteil ist grün hervorgehoben (modifiziert nach Mansour 2001, S. 36).

Nun ergeben sich unterschiedliche Bewegungsmöglichkeiten. Es kann eine Rotation des beweglichen Körpers um seinen eigenen Krümmungsmittelpunkt $M_{\text {bew }}$ stattfinden oder bzw. und um $\mathrm{M}_{\mathrm{fix}}$. Die gemeinsame momentane Drehachse $\mathrm{P}$ kann mithilfe der Winkelgeschwindigkeiten der Teilbewegungen berechnet werden und steht senkrecht auf dem Kettenglied m. Die Rotation kann zusätzlich entweder als Gleit- oder Rollbewegung stattfinden. Der Kontaktpunkt beider Gelenkflächen wird K bezeichnet. Dieser Punkt bleibt auf $\mathrm{G}_{\text {bew }}$ ortsfest und wandert auf $\mathrm{G}_{\mathrm{fix}}$, wenn eine reine Gleitbewegung von $\mathrm{G}_{\mathrm{fix}}$ vorliegt (Abbildung $8 \mathrm{~A}$ ). Bei dieser Bewegung bildet $\mathrm{M}_{\mathrm{fix}}$ die Rotationsachse. Handelt es sich um eine Bewegung um die Achse $M_{\text {bew }}$, bleibt der Kontaktpunkt auf $G_{f i x}$ konstant, während er auf $\mathrm{G}_{\text {bew }}$ mitläuft (Abbildung $8 \mathrm{~B}$ ). Bei einem reinen Abrollen findet eine gleichzeitige Rotation um $\mathrm{M}_{\text {bew }}$ und $\mathrm{M}_{\mathrm{fix}}$ statt. In diesem Fall bewegt sich $\mathrm{K}$ sowohl auf $\mathrm{G}_{\mathrm{fix}}$ als auch auf $\mathrm{G}_{\text {bew }}$ (Abbildung $8 \mathrm{C}$ ).

A

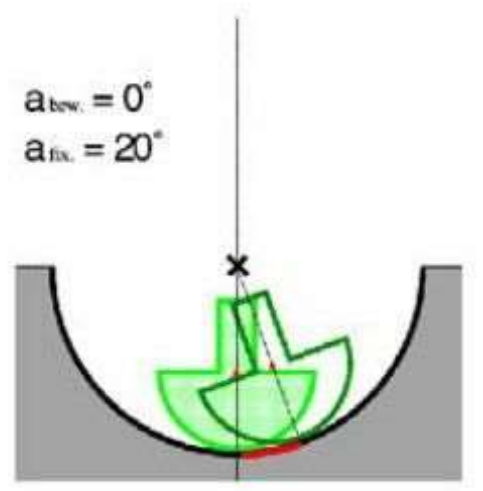

B

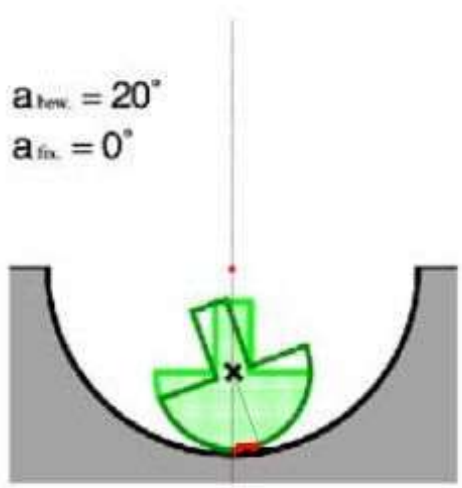

C

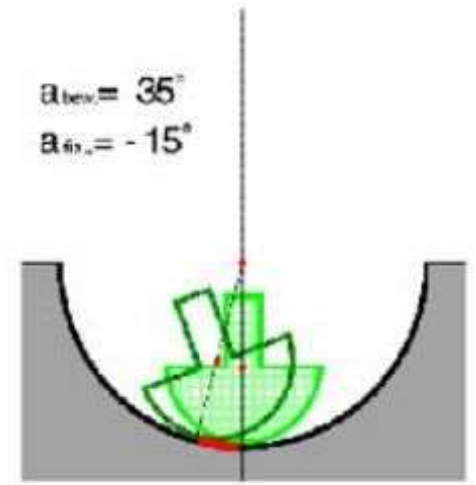

Abbildung 8: Modell einer überschlagenen dimeren Gelenkkette vor und nach einer differentiell kleinen Elementarbewegung. $A$ ) reine Rotation um $\mathrm{M}_{\mathrm{fix}}$ (Gleiten) und $B$ ) reine Rotation um $\mathrm{M}_{\text {bew }}$ (Rollgleiten);

C) Rotation um den wandernden Kontaktpunkt (Rollen). Der bewegte Gelenkteil ist grün hervorgehoben, die Wanderungsstrecke rot. Das X markiert die Rotationsachse (Mansour 2001, S. 37). 
Auch bei der kombinierten Roll-Gleit-Bewegung verändert sich der Kontaktpunkt auf beiden Gelenkflächen, allerdings legt er nun unterschiedliche Wanderungsstrecken zurück. Dominiert die Rotation um $\mathrm{M}_{\text {bew }}$, nähert sich die momentane Drehachse $\mathrm{P}$ an den Kontaktpunkt $\mathrm{K}$ an. Entsprechend entfernt sich $\mathrm{P}$ von $\mathrm{K}$, wenn die Bewegung um $\mathrm{M}_{\mathrm{fix}}$ überwiegt. Liegt eine gleichsinnige Rotation um $\mathrm{M}_{\text {bew }}$ und $\mathrm{M}_{\mathrm{fix}}$ vor, befindet sich $\mathrm{P}$ oberhalb des Kontaktpunktes, bei gegensinniger Bewegung unterhalb.

\subsubsection{Die Viergelenkkette}

Sind beide Zwischenwirbelgelenke eines Wirbelsäulensegments kraftschlüssig, kommt es zu einem Zusammenwirken von zwei dimeren Gelenkketten. In diesem Zustand kann nach Nägerl et al. (1992) das mechanische Prinzip einer symmetrischen Viergelenkkette angewandt werden. Diese besteht entsprechend aus jeweils zwei fixen und beweglichen Körpern $\left(\mathrm{G}_{\mathrm{fix}} 1, \mathrm{G}_{\mathrm{fix}} 2\right.$ und $\mathrm{G}_{\text {bew } 1}$, $\left.\mathrm{G}_{\text {bew }} 2\right)$. Im vorliegenden Beispiel des Wirbelsäulensegments sind dabei die Abstände zwischen den Krümmungsmittelpunkten der beweglichen bzw. fixen Gelenkflächen konstant. Ermittelt man die Kettenglieder jeder dimeren Kette und legt eine zum Kontaktpunkt senkrechte Gerade durch sie hindurch, befindet sich im Schnittpunkt dieser beiden Geraden senkrecht dazu die momentane Drehachse P der Viergelenkkette. Diese Drehachse ist nicht ortsfest, sondern variiert in Abhängigkeit von der Position der Gelenkflächen. Werden nun die Schnittpunkte der Drehachsen und einer definierten Ebene von differentiell kleinen Rotationen graphisch nebeneinander dargestellt, erhält man die Rastpolkurve, also die Wanderung der momentanen Drehachsen während einer Bewegung (Abbildung 9).

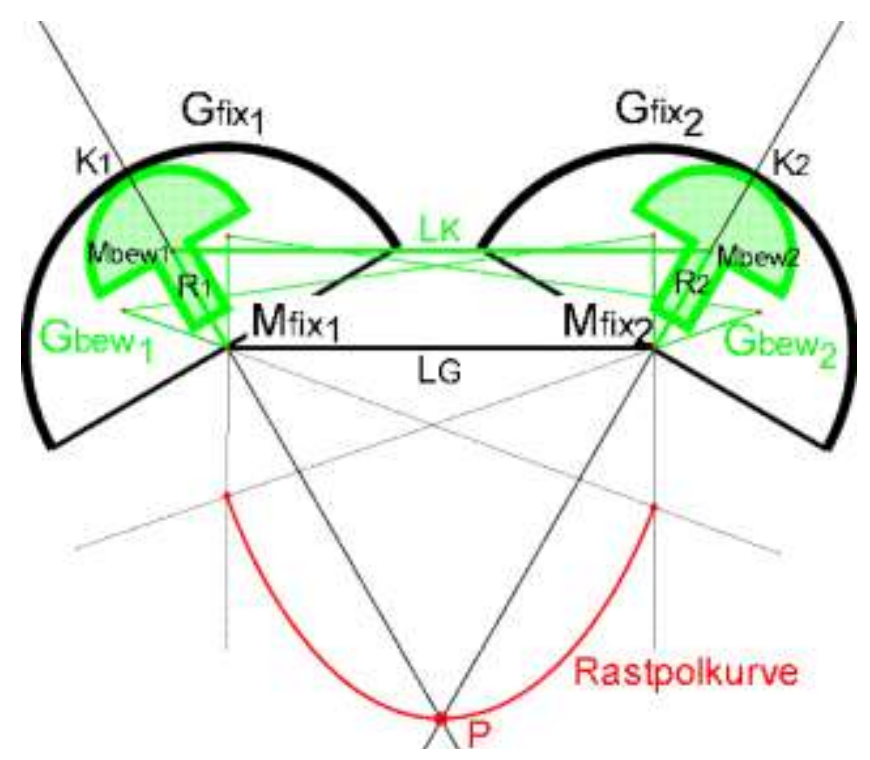

Abbildung 9: Modell einer symmetrischen Viergelenkkette. Der bewegte Teil ist grün hervorgehoben. Für die Krümmungsmittelpunkte (rot) und ihre Verbindungslinien sind die Positionen bei differentiellen Bewegungen geometrisch konstruiert. Der Ort der momentanen Schraubachsen $\mathrm{P}$ und die Rastpolkurve bei differentieller Betrachtung sind rot dargestellt (modifiziert nach Nägerl et al. 1995). 


\section{Material und Methodik}

Das nachfolgende Kapitel befasst sich zunächst mit der Auswahl und Vorbereitung der zu untersuchenden Wirbelsäulenpräparate. Im Verlauf wird ein Überblick über den Aufbau und die Funktionsweise der Versuchsapparatur gegeben sowie das Studiendesign vorgestellt.

\subsection{Lendenwirbelpräparate}

Bei den fünf in dieser Studie verwendeten Präparaten handelt es sich um Autopsiematerial des anatomischen Instituts der Ernst-Moritz-Arndt-Universität Greifswald, das von Prof. Fanghänel zur Verfügung gestellt wurde.

Die in einem Stück entnommenen Wirbelsäulenpräparate (C1-S5 bzw. Th12-S5) wurden zunächst radiologisch zum Ausschluss von Pathologien untersucht und sorgfältig unter Erhalt der Bandstrukturen und Gelenkkapseln freipräpariert. Danach erfolgte die Trennung der L3/L4er Segmente aus dem Wirbelverband. Die Präparate wurden gekühlt aufbewahrt und in einer Formalin-Ethanol-Glycin-Lösung (Fanghänel und Schultz 1962) konserviert, die weder die elastischen Eigenschaften der Bänder noch die Härte der Knochen beeinflusst. Diese Lösung fand, auf eine Kompresse aufgetragen, auch Verwendung, um die Präparate während der Messungen vor dem Austrocknen zu schützen. Die Kennzeichnung der Präparate mit den Buchstaben A bis $E$ verhinderte Verwechslungen während der Messungen.

Anschließend erfolgte die Einbettung der Präparate. Ziel dieser Einbettung war die starre, unbewegliche und reproduzierbare Verbindung des Präparats mit der Messapparatur. Dies wurde über das Eingießen des Präparats in Weitur® Press (Standard) erreicht. Dabei handelt es sich um einen kaltpolymerisierenden Kunststoff, der röntgendicht und gegen Konservierungslösungen beständig ist. Als Verbindungsglied zwischen den eingegossenen Präparaten und der Messapparatur fungierte dabei eine Stahlwanne, die gleichzeitig die Gussform für den Kunststoff darstellte.

Zum Einbetten wurde zunächst die Stahlwanne von außen mit den vier Schrauben versehen, die später der Befestigung an der Apparatur dienten. An die Schrauben wurden inwändig Flügelmuttern angebracht, die einen besseren Halt im Kunststoff sowie die Führung der Schrauben gewährleisteten. Das Spicken der Wirbel mit Schrauben ermöglichte die genaue Ausrichtung des Segments in der Wanne und verbesserte die Verankerung im Kunststoff. Dabei war darauf zu achten, dass die Schrauben nicht in die Bandscheibe oder die Wirbelbogengelenke eindrangen. Die Positionierung der Präparate in der Wanne erfolgte so, dass sich der Mittelpunkt des Wirbelkanals mit dem der Wanne deckte. Außerdem war die Bandscheibe waagerecht ausgerichtet. Die Stahlwanne wurde im Anschluss vollständig mit dem Kunststoff gefüllt, Bandscheibe und Wirbelbogengelenke blieben vom Kunststoff unberührt. Diese Vorgänge wurden sowohl am kranialen als auch am kaudalen Ende des Segments durchgeführt. Eine 
Führungsschiene diente der möglichst deckungsgleichen Ausrichtung der beiden Stahlwannen.

Eine erneute computertomographische Untersuchung der eingebetteten Präparate bestätigte die Unversehrtheit der Bandscheiben und Gelenkfortsätze (Abbildung 10-14).
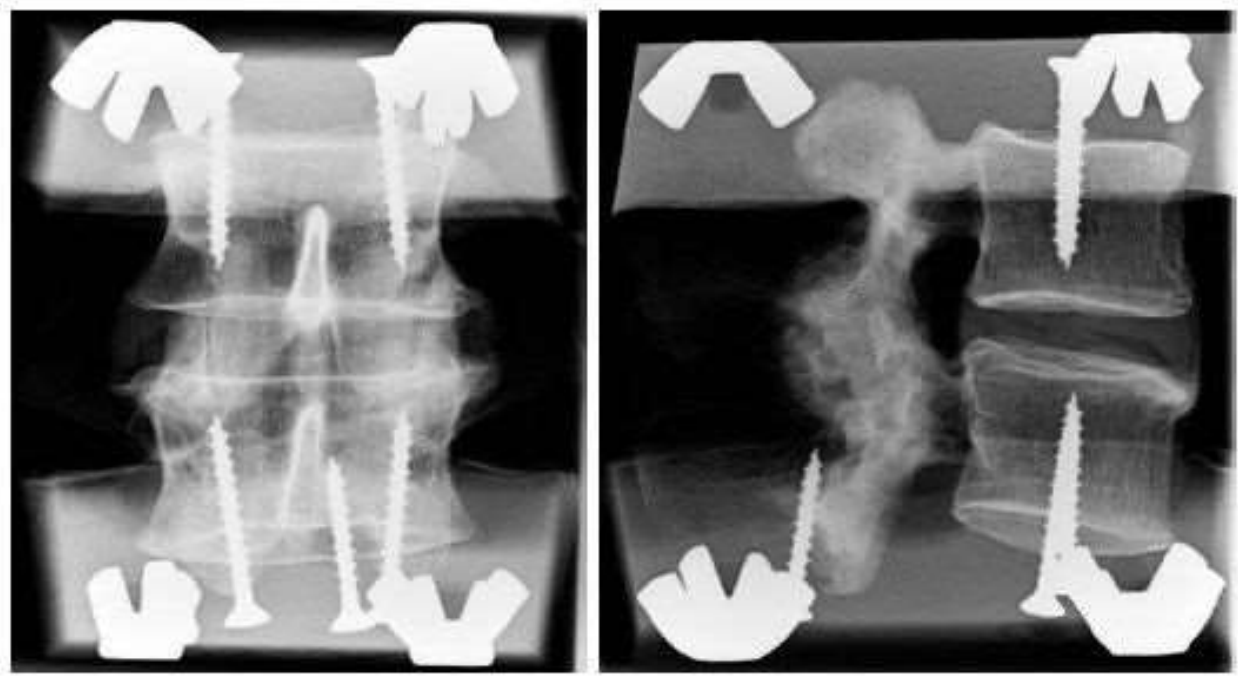

Abbildung 10: Röntgen des eingebetteten Präparats A im ap- (anterior-posterioren, links) und lateralen (rechts) Strahlengang
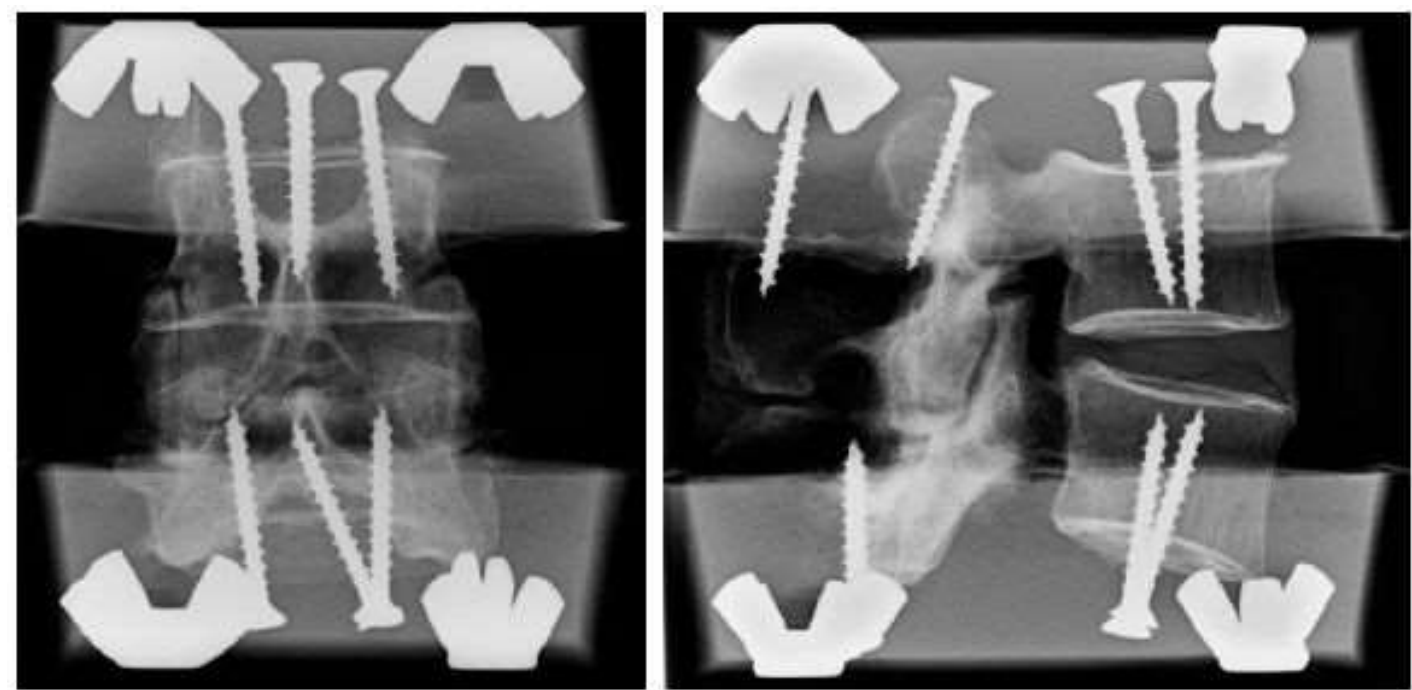

Abbildung 11: Röntgen des eingebetteten Präparats B im ap-Strahlengang (links) und lateralen Strahlengang (rechts) 

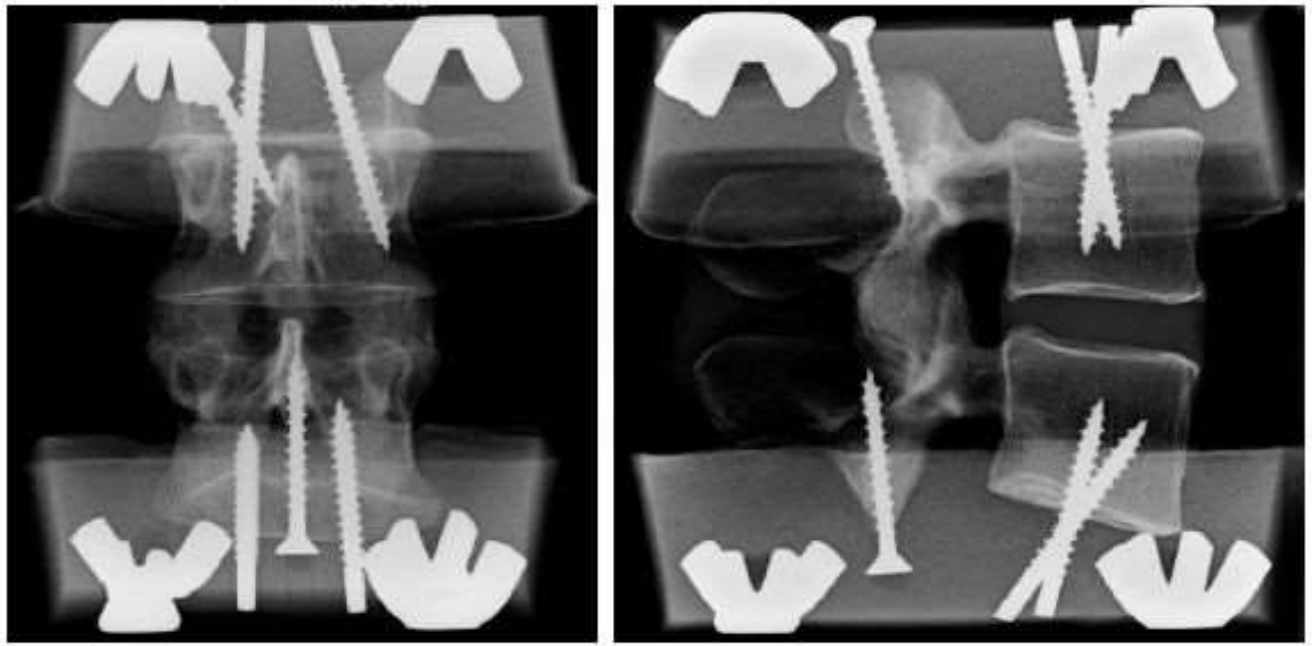

Abbildung 12: Röntgen des eingebetteten Präparats C im ap-Strahlengang (links) und lateralen Strahlengang (rechts)
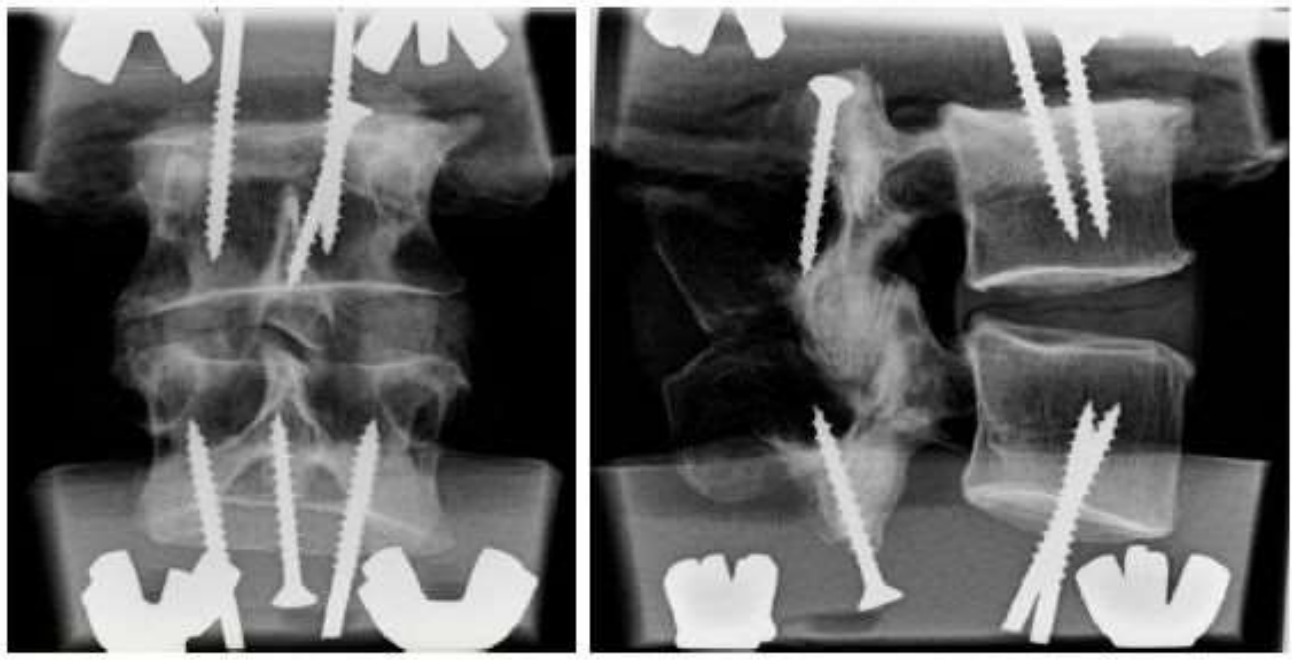

Abbildung 13: Röntgen des eingebetteten Präparats D im ap-Strahlengang (links) und lateralen Strahlengang (rechts)
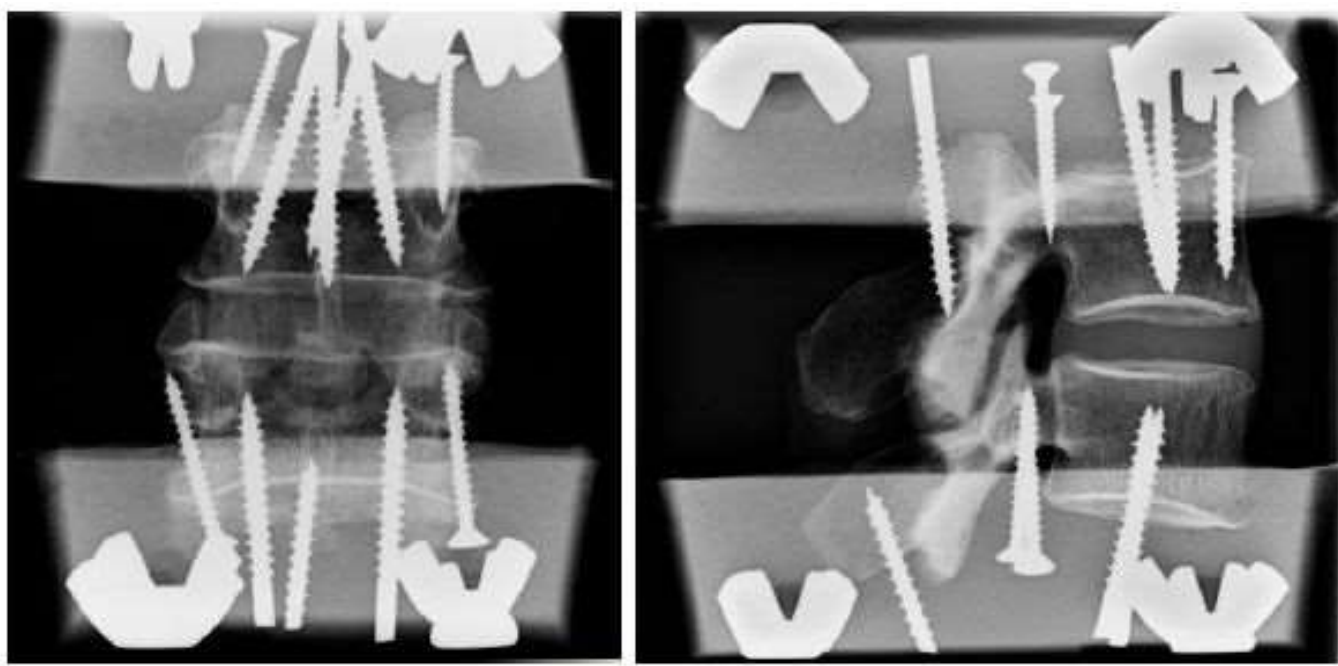

Abbildung 14: Röntgen des eingebetteten Präparats E im ap-Strahlengang (links) und lateralen Strahlengang (rechts) 


\subsubsection{Analyse der Gelenkfortsätze}

In der radiologischen Bildgebung waren keinerlei pathologische Veränderungen der Wirbelbogengelenke festgestellt worden. Bemerkenswert war jedoch die Variabilität der Ausrichtung der Gelenkflächen, auch Facetten genannt. So zeigten sich Abweichungen zur Sagittalen von $25-50^{\circ}$, bzw. Facettenwinkel $\varphi$ von $50-100^{\circ}$, die die Facetten der linken und rechten Gelenke zueinander aufwiesen (Abbildung 15 und Tabelle 1). Weiterhin ist zu bemerken, dass die konvexe Wölbung des linken Wirbelbogengelenks des Präparats B gegenüber der des rechten abgeflacht war (Abbildung 15B). An den Präparaten B und insbesondere A fällt auf, dass die medial gelegenen Gelenkfortsätze von den lateralen halb umschlossen wurde, was bei den übrigen Präparaten nicht der Fall war (Abbildung 15A und B).

Tabelle 1: Facettenwinkel $\varphi$ aller Präparate

\begin{tabular}{|c|c|c|c|c|c|}
\hline Winkelposition/Präparat & A & B & C & D & E \\
\hline Gesamtwinkel $\varphi$ & $50^{\circ}$ & $70^{\circ}$ & $70^{\circ}$ & $100^{\circ}$ & $80^{\circ}$ \\
\hline $\begin{array}{c}\text { Abweichung von } \\
\text { Sagittalachse nach rechts }\end{array}$ & $25^{\circ}$ & $32^{\circ}$ & $30^{\circ}$ & $50^{\circ}$ & $40^{\circ}$ \\
\hline $\begin{array}{c}\text { Abweichung von } \\
\text { Sagittalachse nach links }\end{array}$ & $25^{\circ}$ & $38^{\circ}$ & $40^{\circ}$ & $50^{\circ}$ & $40^{\circ}$ \\
\hline
\end{tabular}
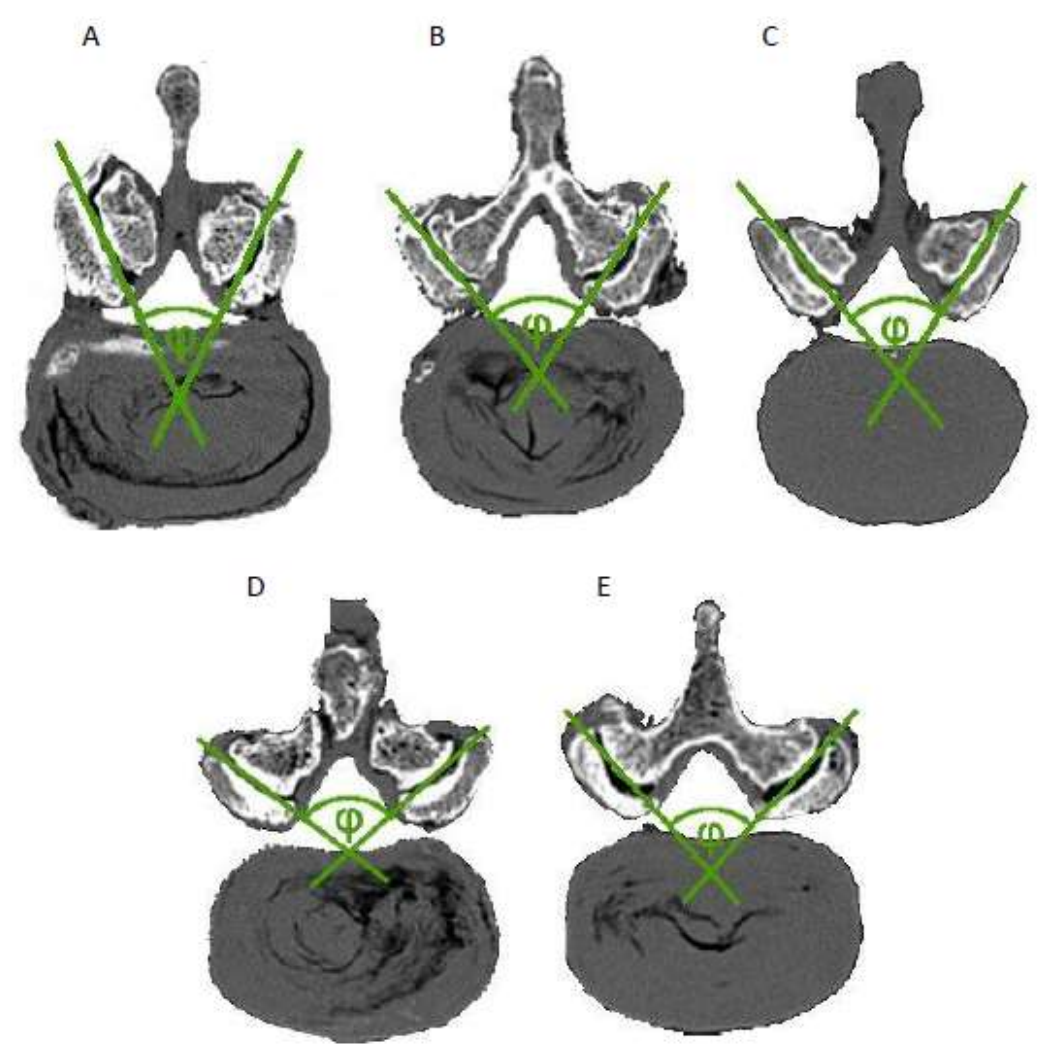

Abbildung 15: Transversale Schnittbilder der Präparate A-E (entsprechen den Bildern A-E) mit eingezeichnetem Facettenwinkel $\varphi$. Der Facettenwinkel ist trotz gleicher Segmenthöhe variabel. 
Auch in der kranio-kaudalen Ausrichtung der Gelenkfacetten zeigten sich Abweichungen (Winkel $\omega 1$ bzw. $\omega 2$ ), in diesem Fall von einer vertikalen Linie (Abbildung 16). Der Winkel variierte zwischen $-10^{\circ}$ und $11^{\circ}$ für die rechten Wirbelbogengelenke (Winkel $\omega 1$ ) und zwischen $-4^{\circ}$ und $15^{\circ}$ für die linken Gelenke (Tabelle 2). Positive Winkel bedeuten eine Abweichung nach links, negative nach rechts. Auffällig ist am Präparat $C$, dass die Ausrichtung der Facettengelenke von kranial-medial nach kaudal-lateral verläuft, während sie bei allen anderen Präparaten gegenläufig (also von kranial-lateral nach kaudal-medial) ist (Abbildung 16C).

Tabelle 2: Abweichung der Ausrichtung der Gelenkfacetten von einer vertikalen Linie in ${ }^{\circ}$. Positive Vorzeichen bedeuten eine Abweichung nach links, negative nach rechts.

\begin{tabular}{|c|c|c|c|c|c|}
\hline $\begin{array}{c}\text { Winkelposition/Präparat } \\
\text { rechtes Wirbelbogenge- } \\
\text { lenk }\end{array}$ & $-6^{\circ}$ & $-10^{\circ}$ & $11^{\circ}$ & $-9^{\circ}$ & $-10^{\circ}$ \\
\hline linkes Wirbelbogengelenk & $3^{\circ}$ & $9^{\circ}$ & $-4^{\circ}$ & $15^{\circ}$ & $4^{\circ}$ \\
\hline
\end{tabular}

A

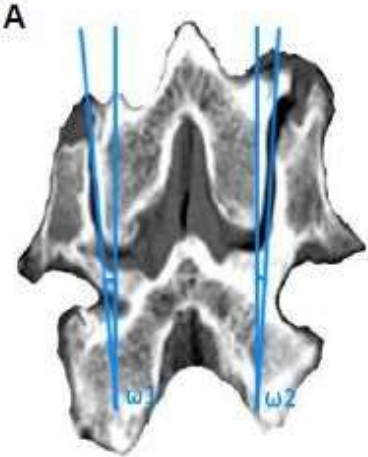

D

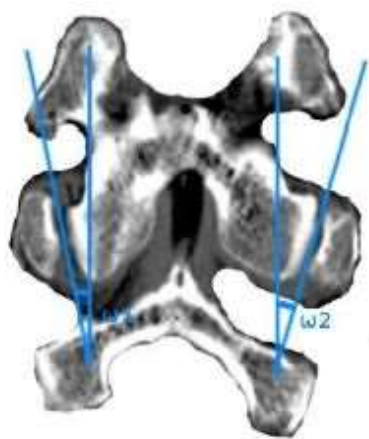

B

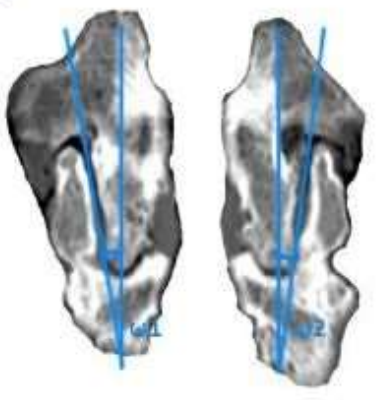

C

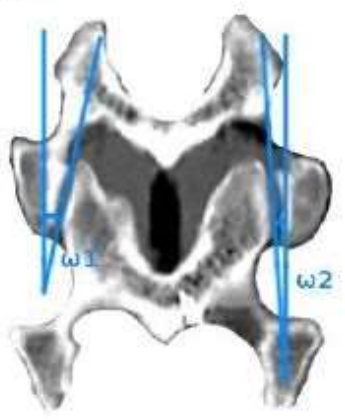

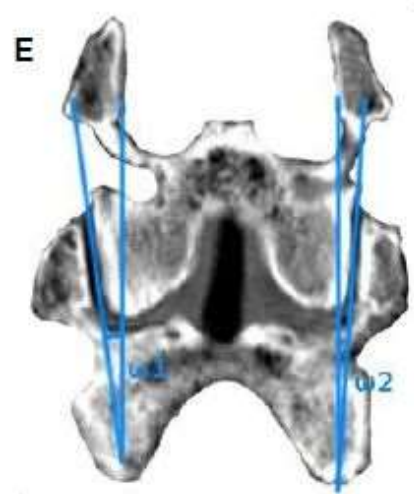

Abbildung 16: Coronale Schnittbilder durch die Wirbelbogengelenke der Präparate A-E (entsprechen den Bildern A-E) mit eingezeichnetem Abweichungswinkel $\omega$ der Gelenkfacetten von einer vertikalen Linie. $\omega 1$ : rechtes Gelenk; $\omega 2$ : linkes Gelenk. Ansicht von ventral. Der Winkel ist trotz gleicher Segmenthöhe variabel. 


\subsection{Versuchsaufbau}

Grundlage dieser Studie ist eine biomechanische Analyse eines Bewegungssegments der Höhe L3/L4 von fünf Lendenwirbelsäulen. Zur Durchführung dieser Analyse werden verschiedene Kräfte (s. Kapitel 2.2.2) an das Segment angelegt. Durch die Kräfte erfolgt eine Verkippung der Wirbel zueinander. Dies wiederum kann zur Folge haben, dass sich die Voraussetzungen der Bewegungsstruktur durch Kopplung oder Entkopplung und Verschiebung der Kontaktpunkte im Gelenksystem ändern (Nägerl et al. 1995). Lagesensoren zeichnen die Bewegung des oberen Wirbels auf, ein Messprogramm stellt sie grafisch dar. Nach der Messung am intakten Segment wird zunächst das rechte, dann auch das linke Wirbelbogengelenk entfernt und die Messungen nach jedem Resektionsschritt wiederholt. Durch den Vergleich der Ergebnisse vor und nach den Resektionen können Rückschlüsse auf den Einfluss der Wirbelbogengelenke auf die Bewegungsvorgänge gezogen werden, da Versuchsaufbau und -umsetzung mit Ausnahme der Resektion unverändert bleiben.

Zur experimentellen Umsetzung müssen bestimmte Voraussetzungen gegeben sein. So ist der L4-Wirbel starr mit dem Messstand zu verbinden, während der L3-Wirbel im Raum frei beweglich sein muss. Eine stete Reproduzierbarkeit der Kräfte ist erforderlich. Die Messung der Positionsveränderung des L3-Wirbels erfolgt hochaufgelöst in praktisch differentiell kleinen Schritten, so dass jeder dieser Schritte in sehr guter Näherung als momentane Schraubbewegung dargestellt und der tatsächliche Bewegungsvorgang rekonstruiert werden kann.

\subsubsection{Messstand}

Der Messstand (Spiering 1995, Abbildung 17) besteht zum einen aus einem Metallrahmen aus Vierkantstangen, der weitgehende Schwingungsfreiheit gewährt, da er auf Schaumstoff gelagert ist und die einzelnen Stangen mit Schaumstoff ummantelt sind. Über eine Metallgrundplatte mit aufmontierter Stahlwanne ist er mit der eigentlichen Messapparatur verbunden. Das zu messende Präparat wird in sagittaler Richtung in der Wanne verschraubt, wodurch eine fixe Verbindung des Lendenwirbels L4 mit dem Messstand gegeben ist. Das kraniale Ende des Präparats wird an der zweiten Stahlwanne befestigt, die mit einem metallenen Kreuz verschweißt ist. Dieses Drehkreuz überträgt die an ihm ansetzenden Drehmomente und axialen Vorlasten auf den Lendenwirbel L3 (s. Kapitel 2.2.2), der dadurch verkippt. Ein umgelenktes Gegengewicht hebt die Gewichtskraft des Drehkreuzes auf, so dass kein Druck auf das darunterliegende Präparat einwirkt. Die Messtaster, die die Verkippung des oberen Wirbels registrieren, sind in einen Rahmen eingelassen, der das zu messende Präparat umschließt und eine unveränderliche Position der einzelnen Messtaster zueinander gewährleistet. Der Messtasterrahmen steht über Führungsschienen, die eine Höhenverschiebung erlauben, ebenfalls mit der Grundplatte in Verbindung. 


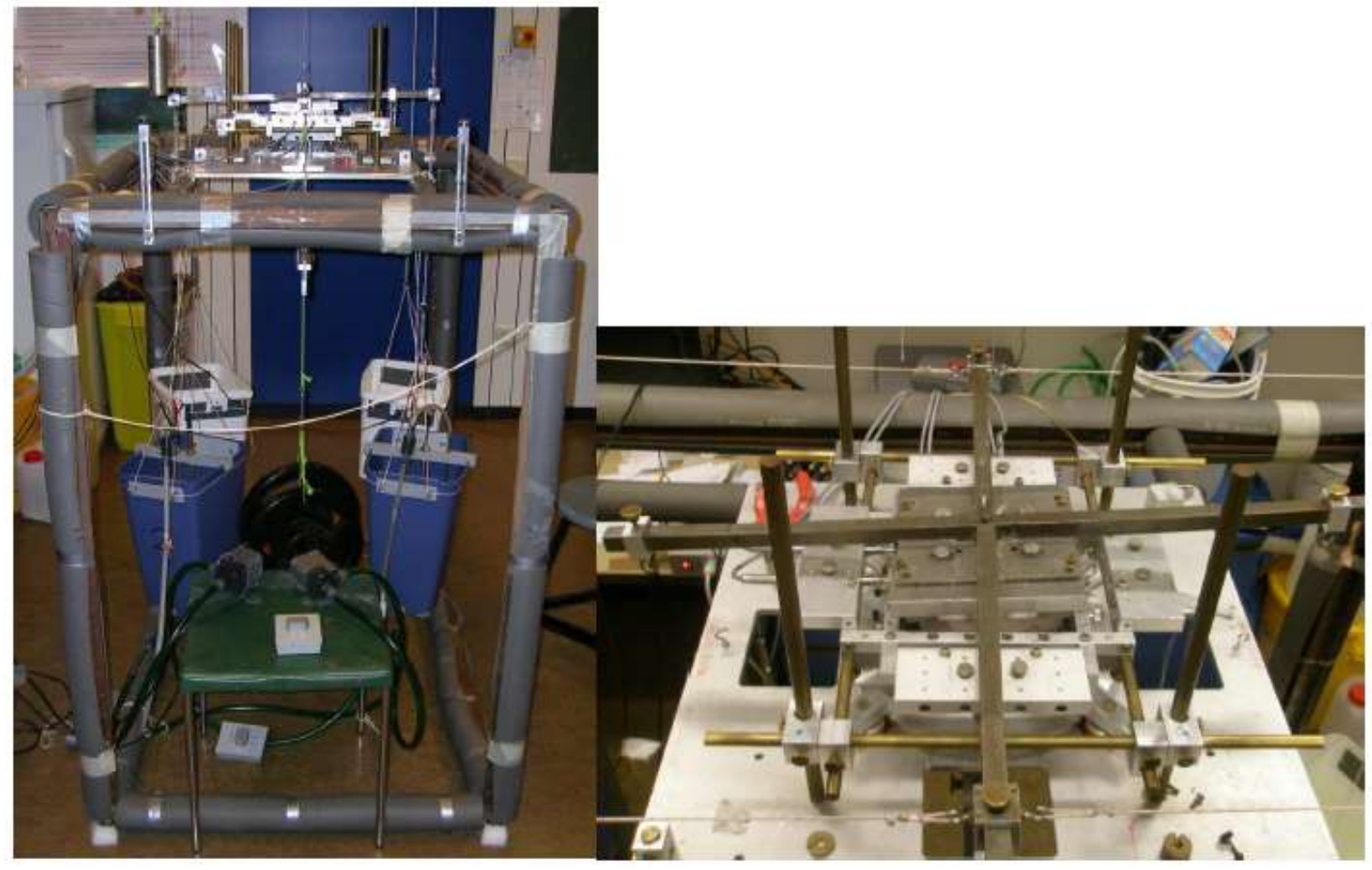

Abbildung 17: Messstand (links) und Drehkreuz (rechts)

\subsubsection{Applikation der Kräfte}

Das Prinzip dieser Studie liegt in der zeitgleichen Einwirkung einer axial gerichteten Kraft (Vorlast) sowie eines zyklischen Drehmoments, das je nach Versuchsaufbau zu einer Rotation um die x-, y- oder z-Achse führt.

Um die Vorlast zu applizieren, werden an gegenüberliegenden Armen des Drehkreuzes Zugseile angebracht, an denen wiederum eine Schiene mit einem verschiebbaren Reiter hängt. Die Ausrichtung der Schiene erfolgt in x- oder y-Richtung. Allein durch die Schiene erfährt das Segment eine zentrale kompressive Belastung von 10 N. Zusätzlich können an den Reiter weitere Gewichte angehängt werden, die in ihrer Position variabel sind. Die Projektion der Vorlastpositionen auf den Wirbel zeigt Abbildung 18. Eine Millimeterskala auf der Schiene erlaubt die genaue Platzierung des Reiters (Abbildung 19). Der Vorteil dieser Konstruktion liegt darin, dass sie im Raum frei beweglich ist und dadurch den Bewegungen des Segments folgen kann ohne den Bewegungsvorgang zu beeinflussen. Die Vorlast simuliert das Gewicht des Rumpfes, das auf der Lendenwirbelsäule lastet. Durch die Verschiebung der Vorlast können nicht nur der aufrechte Stand, sondern auch Haltungen nachgeahmt werden, die bei Beugung des Körpers nach ventral und dorsal sowie nach lateral entstehen. Je nachdem, wo die Vorlast angebracht wird, resultiert neben einer reinen Kompression des Segments auch eine unterschiedlich stark ausgeprägte Neigung des oberen Wirbelkörpers. 


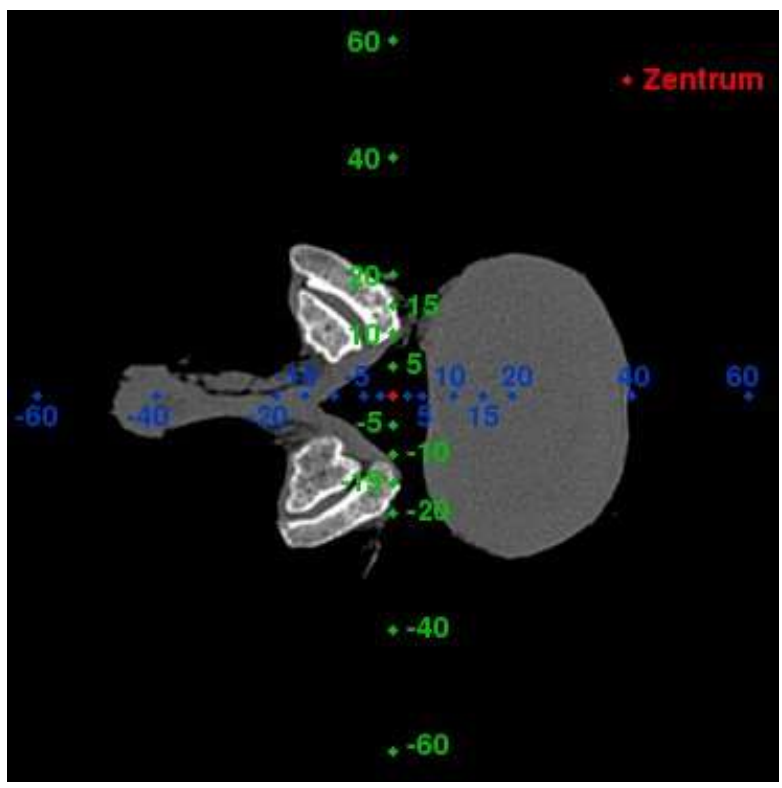

Abbildung 18: Gewählte Positionen zur Anbringung der Vorlast. Das Zentrum liegt in der Mitte des Wirbelkanals. Die Bezeichnung der Positionen bezieht sich auf die Entfernung (in mm) vom Zentrum.

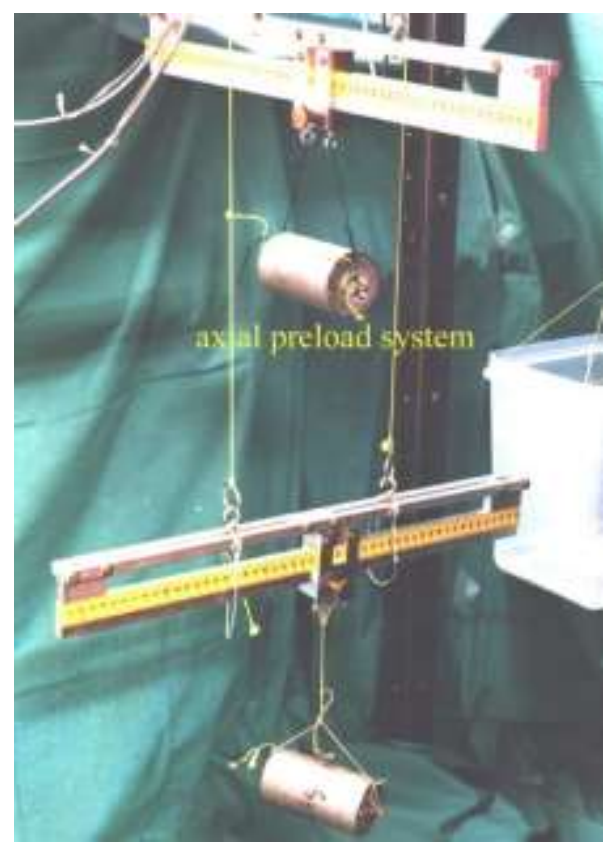

Abbildung 19: Prinzip der Anbringung der Vorlast an verschieblichen Reitern (Mansour 2001, S. 60)

Das Drehmoment wird ausgeübt, indem je ein Gewicht über einen Seilzug auf zwei gegenüberliegende Arme des Kraftkreuzes wirkt. Die Gewichte bestehen dabei aus mit Wasser gefüllten Behältern. Über Pumpen können Wassermassen zwischen den beiden Behältern hin und her verschoben werden, so dass das Drehmoment zyklisch geändert wird. Zunächst sei der Aufbau für die Ausübung eines axialen Drehmoments (in $\mathrm{T}_{\mathrm{Z}}$-Richtung) in der Gleichgewichtsstellung erläutert.

Es werden vier Seile an den in y-Richtung weisenden Armen des Drehkreuzes befestigt und so ausgerichtet, dass sie im 90-Winkel nach ventral (zwei Seile) bzw. dorsal (ebenfalls zwei Seile) führen. Die Umlenkung erfolgt über kugelgelagerte Rollen, die in 
mindestens $50 \mathrm{~cm}$ Entfernung zum Drehkreuz am Rahmen der Messapparatur angebracht sind. An jeden dieser Seilzüge wird ein gefüllter Wasserbehälter gehängt. Da jeder Behälter nun gleich schwer ist, heben sich jegliche Kräfte durch Antiparallelität auf, das System befindet sich im Gleichgewicht. Verbindet man die dorsal des Messstands hängenden Behälter mit Wasserpumpen, kann das Wasser beispielsweise von dem rechten in den linken Behälter gepumpt werden. Dadurch entsteht an dem linken Arm des Kraftkreuzes ein Zug nach dorsal, also ein reines Drehmoment in z-Richtung (Linksdrehung). Durch das gewählte Prinzip der Kräftegleichheit wird gewährleistet, dass unabhängig von der Größe des Drehmoments die Summe der vier Kräfte stets null ergibt (Abbildung 20).

Der Aufbau für Drehmomente in $x$ - und $y$-Richtung erfolgt analog, abweichend ist nur die Position der Wasserbehälter. Die zwei mit dem Pumpsystem verbundenen Behälter werden direkt an die Kraftkreuzarme in $y$ - (Drehmomente in $x$-Richtung) bzw. $x$-Richtung (Drehmomente in y-Richtung) gehängt. Die Zugseile für die Gegengewichte zum Kräfteausgleich werden senkrecht nach oben geführt und in der Höhe umgelenkt. Auch in diesem Fall ist die Konstruktion somit kräftefrei.

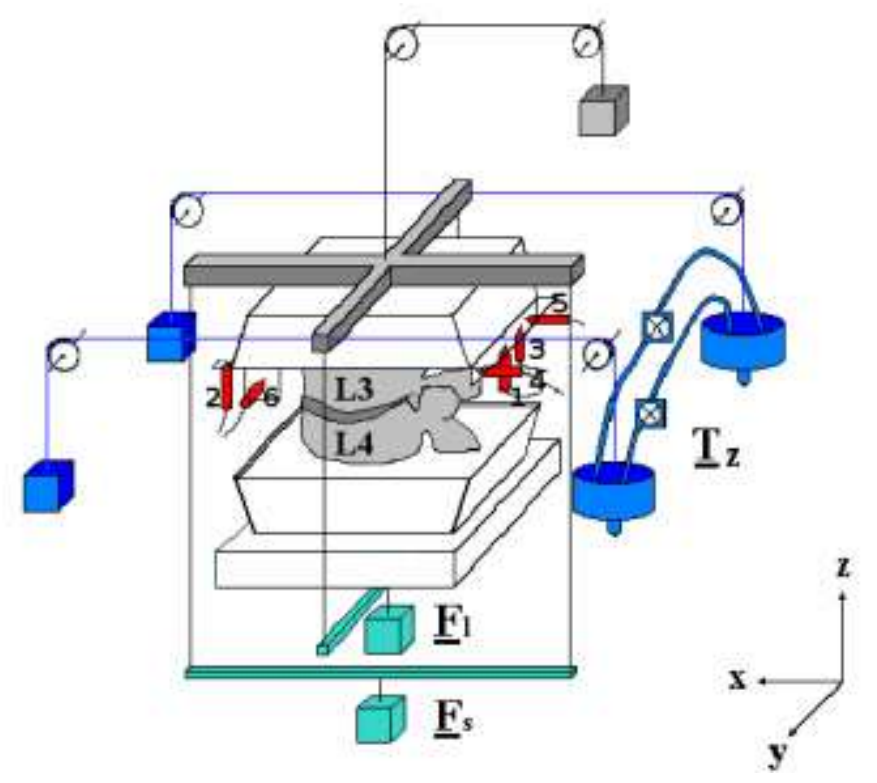

Abbildung 20: Skizze der Messapparatur. Die Vorlast ist türkis, das Drehmomentsystem mit den Wasserbehältern blau und die nummerierten Messtaster sind rot markiert. Das Wirbelsäulensegment ist in grau eingezeichnet (modifiziert nach Mansour 2001, S. 57).

Die zwei Pumpen der Firma Eheim Typ 8060 haben ein Fördervolumen von 4,5 I/min und werden luftleer betrieben. Sie haben entgegengesetzte Förderrichtungen, d.h. Pumpe 1 pumpt das Wasser von dem linken in den rechten Behälter, Pumpe 2 aus dem rechten in den linken. Das Pumpsystem ist mit einer elektronischen Steuerung versehen: An die Behälter sind je zwei Elektroden angebracht, die den Wasserstand während der Versuchsdurchführung messen. Ist die minimale Füllmenge erreicht, verliert eine der beiden Elektroden den Kontakt mit dem Wasser und der Stromkreis unterbricht. Durch dieses Signal schaltet die Steuerung (Spiering 2002) von der einen auf 
die andere Pumpe um. Die Restwassermenge beträgt 1,5 Liter und verhindert das Leerlaufen des Systems. Ein Pumpzyklus entspricht der Beförderung der kompletten Wassermenge von einem Behälter in den anderen. Im Gegensatz dazu besteht ein Messzyklus aus zwei Pumpzyklen. Das heißt beispielsweise bei Rotation um die z-Achse, dass der Wirbel von der maximal nach links rotierten Stellung eine Rechtsdrehung vollführt (erster Pumpzyklus) und nach Erreichen des Maximums wieder in die linksrotierte Position zurückkehrt (zweiter Pumpzyklus).

Zur Fehlerabschätzung bei der Erzeugung des Drehmoments siehe Anhang A.

\subsubsection{Messtaster, Messinterface und Software}

Für diesen Versuch werden sechs Messtaster benötigt, da der obere Wirbel sich in sechs möglichen Richtungen bewegen kann, d.h. er besitzt sechs Freiheitsgrade: eine Translation entlang sowie eine Rotation um jede der drei Raumachsen. Für die Detektion dieser Bewegungen wurde eine '3-2-1-Anordnung' der Messtaster gewählt (Abbildung 21). Sie ergibt sich aus folgenden Überlegungen: Für die $x-y$-Ebene braucht man einen Taster, der die Translation misst, sowie je einen weiteren für Rotationen um die $x$ - und y-Achse - diese entsprechen den Tastern 1, 2 und 3 in der Messapparatur. In der y-z-Ebene benötigt man wiederum einen Taster für die Translation und einen für die Rotation um die z-Achse - Taster 4 und 5. Da die Rotation um die y-Achse bereits durch die Taster 1-3 detektiert wird, genügen hier die erwähnten Taster 4 und 5 . Nun muss nur noch die Translation entlang der x-z-Ebene aufgenommen werden, was durch den Taster 6 geschieht. Durch die Messergebnisse dieser sechs Taster kann die Veränderung der Lage des oberen Wirbels exakt berechnet werden.

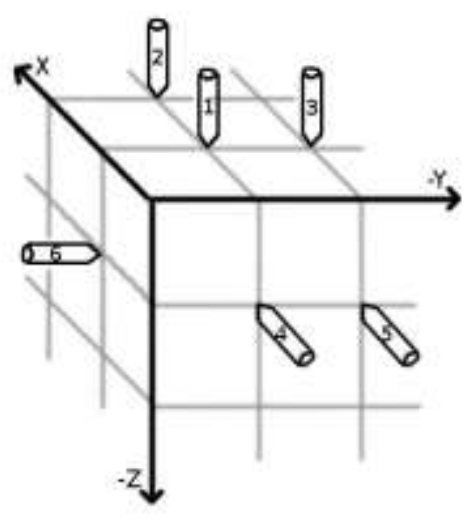

Abbildung 21: Räumliche 3-2-1-Anordnung der sechs Messtaster

Die in dieser Studie verwendeten Lagesensoren (Fa. Mahr GmbH Göttingen, Typ 1310) folgen dem Prinzip der induktiven Längenmessung: Ein Metallstift ist in der Tasterummantelung auf einer Feder gelagert. Bei Ausübung leichten Drucks auf die Spitze des Stifts wird dieser weiter in die Ummantelung geschoben, bei Nachlassen des Drucks sorgt die Feder für eine gegensinnige Bewegung. Die Messtasterspitze ist ab- 
gerundet und gleitet auf Glasplättchen, die an dem Drehkreuz angebracht sind; durch die feste Verbindung des Messobjekts mit dem Drehkreuz kann man davon ausgehen, dass die Lageänderung des Kreuzes der des oberen Wirbels entspricht. Aufgrund des Glasplättchens wird eine elastische Verformung der Tasterspitze verhindert und eine plane Kontaktoberfläche gesichert. Der Anpressdruck und die Reibung werden weitestgehend minimiert. Die Taster werden zu Beginn jeder Messung auf halbe Federspannung gestellt, um Auslenkungen in positiver (Tasterverkürzung) und negativer (Tasterverlängerung) Richtung wahrzunehmen und in Spannungswerte umzuwandeln. Die Auflösung beträgt $0,01 \mu \mathrm{m}$.

Über das Messinterface-System Millimar X1715 der Firma Mahr werden alle Daten der sechs Messtaster simultan aufgenommen und an einen handelsüblichen PC weitergeleitet. Die dort installierte Software Millimar D1000x dient der Steuerung des InterfaceSystems, ein mit LabView® (National Instruments) erstelltes Programm der Weiterverarbeitung der Daten, d.h. der Berechnung und Darstellung der Lageveränderung des Wirbels.

\subsubsection{Präzisionsschraube und Bandscheibenmodelle}

Zur Kalibrierung und Überprüfung der Zuverlässigkeit der Messapparatur wurden Messungen mit Objekten definierter Eigenschaften durchgeführt. Der Versuchsaufbau folgte den Angaben des Experiments „Axialrotation“. Das Drehmoment wurde jedoch manuell angelegt. Zunächst fanden Messungen mit einer Präzisionsschraube statt (Abbildung 22). Hierzu wurde die Schraube mit der Längsachse in z-Richtung fest an der Apparatur angebracht. Die Position der Schraube wurde so gewählt, dass die Längsachse im Ursprung des Bezugskoordinatensystems lag. Im Anschluss an die Messungen fand ein Vergleich der gemessenen Ergebnisse mit den durch die Fertigungsmerkmale erwarteten Daten statt (s. Kapitel 3.1.1).

Es folgte die Messung eines Bandscheibenmodells. Dabei handelte es sich um zwei harte PVC-Blöcke, die mit einem homogen-elastischen Gummipuffer miteinander verbunden waren. Der Gummipuffer war kreisrund mit einem Durchmesser von $4 \mathrm{~cm}$ und soll ein vereinfachtes Modell der Bandscheibe darstellen. Die PVC-Blöcke wurden über die entsprechenden Wannen mit der Messapparatur verbunden, so dass der Ursprung des Koordinatensystems im Mittelpunkt des Gummipuffers lag. Die Längsachse des Puffers verlief parallel zur z-Achse.

In einer dritten Messung wurden an das oben beschriebene Bandscheibenmodell Metallstifte installiert, die der Simulation der beiden Zwischenwirbelgelenke dienen sollten (Abbildung 22). Es wurden jeweils zwei Metallstifte an gegenüberliegenden Positionen am oberen und unteren PVC-Block befestigt, der Kontakt der gegenüberliegenden Stifte fand auf Höhe des Gummipuffers statt. Die Stifte, die an der oberen Wanne befestigt waren, endeten in einer Kugel mit einem Durchmesser von $1 \mathrm{~cm}$. Die Stifte an der unteren Wanne münden in einem Plateau von 1,6 cm Durchmesser, dessen Rand im dorsalen Abschnitt über einem Bereich von ca. $2 \times 4 \mathrm{~mm}$ hochgezogen war (Höhe 
$1 \mathrm{~cm}$ ). Die Gelenkmodelle befanden sich $0,8 \mathrm{~cm}$ lateral (jeweils rechts und links) und 2,7 cm dorsal des Mittelpunkts des Gummipuffers.

$\mathrm{Zu}$ den Ergebnissen der Messungen der Bandscheibenmodelle vergleiche Kapitel 3.1.2.

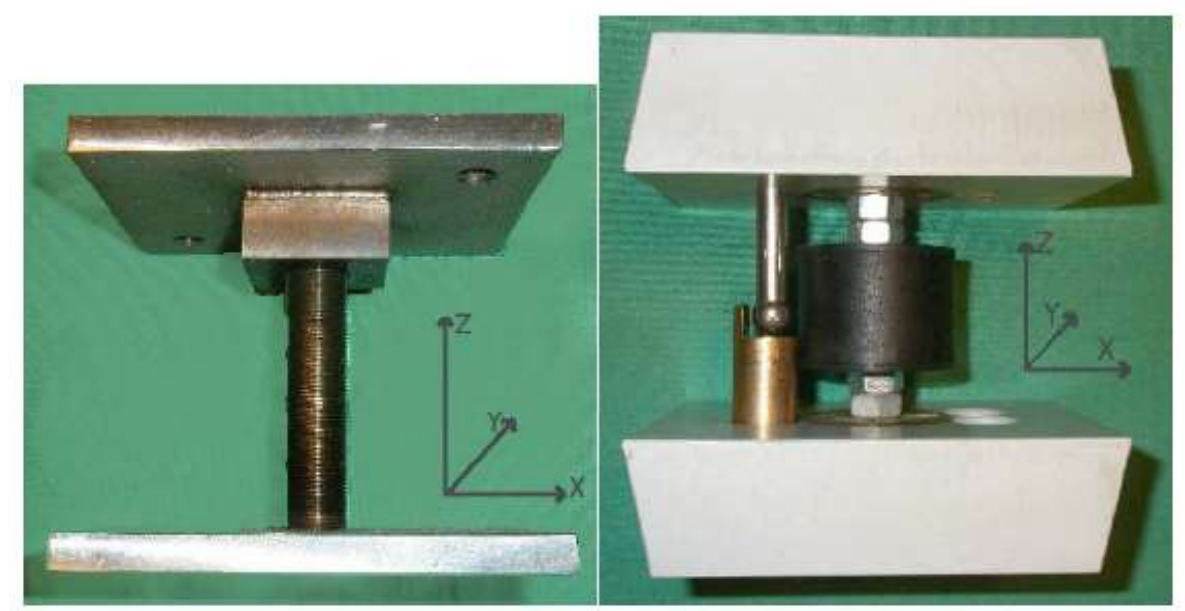

Abbildung 22: Präzisionsschraube (links) und Bandscheibenmodell mit Gelenkmodellen (rechts)

\subsection{Konzept der Schraubachsen}

In dieser Studie wird nicht die Bewegung des oberen Wirbels als Ganzes, also von Position A nach B, betrachtet, sondern als Einheit vieler differentiell kleiner Lageänderungen. Als differentiell klein wird eine Positionsänderung von $0,01^{\circ}$ betrachtet. Da jede Bewegung auf eine Translations- und eine Rotationskomponente zurückgeführt werden kann (s. Kapitel 1.3.1), muss es auch eine Achse geben, um die die Bewegung stattfindet: die Schraubachse. Man kann davon ausgehen, dass die Achse der Gesamtbewegung (kürzester Weg von Position A nach B) nicht zwangsläufig mit der der differentiell kleinen Lageänderungen übereinstimmt. Daher wird der Begriff der momentanen Schraubachse verwendet, wenn die Achse der Teilbewegung gemeint ist; die Schraubachsenschar beschreibt die Gesamtheit der momentanen Schraubachsen. Durch die hohe Frequenz der Bestimmung der Schraubachsen wird eine nahezu lückenlose Aufzeichnung des Bewegungsvorganges erreicht.

Zur Bestimmung der Positionsänderung bzw. der Berechnung der Schraubparameter siehe Anhang B bzw. C.

\subsection{Darstellung der Messergebnisse}

Die ermittelten und verarbeiteten Daten werden mithilfe unterschiedlicher Grafiken, Diagrammen und Tabellen vorgestellt und verdeutlicht. Es handelt sich dabei in der 
Regel um die Ergebnisse eines kompletten Messzyklus im eingeschwungenen Zustand. In den folgenden Abschnitten erfolgt eine Einführung in die gewählten Darstellungstypen, um im Verlauf der Arbeit das Verständnis zu erleichtern.

\subsubsection{Rastpolkurve}

In diesem Diagramm werden die Schnittpunkte $R_{i}$ mit den Koordinaten $x_{i}, y_{i}$ und $z_{i}$ aller momentanen Schraubachsen mit einer definierten Ebene dargestellt. Bei den Versuchen zur Axialrotation ist diese Ebene horizontal ausgerichtet und befindet sich auf Höhe der Messtaster. Die Medianebene stellt die Schnittfläche bei VentralflexionsDorsalextensions-Versuchen dar und eine Frontalebene durch den Mittelpunkt des Messtasterrahmens die der Messungen bei Lateralflexion. Um diese Grafik leichter verständlich zu machen wird ein der jeweiligen Ebene entsprechendes computertomographisches Schnittbild des Präparats in das Diagramm hineinprojiziert. Dies ermöglicht eine exakte räumliche Zuordnung der erhaltenen Ergebnisse zu den realen anatomischen Strukturen. Es ist anzumerken, dass für die Darstellung der Rastpolkurven bei Axialrotation eine Schnitthöhe gewählt wurde, die unterhalb der eigentlichen Messhöhe liegt. Diese Maßnahme erfolgte, um die Zuordnung zum Wirbelkörper und den Wirbelgelenken zu erleichtern. Mit diesen Abbildungen wird zusammenfassend die Position der momentanen Schraubachsen im zeitlichen Verlauf dargestellt (Abbildung 23). Das Kreuz markiert den Anfang der Kurve und das $X$ den Zeitpunkt, zu dem das Segment im nichtrotierten Zustand vorliegt (Drehwinkel $\alpha=0^{\circ}$ ). Weiterhin wurden beschriftete Markierungen an den Positionen gesetzt, an denen eine Rotation von $+1^{\circ}$ bzw. $-1^{\circ}$ vorgelegen hat, um den Bereich des physiologischen Bewegungsumfangs hervorzuheben.

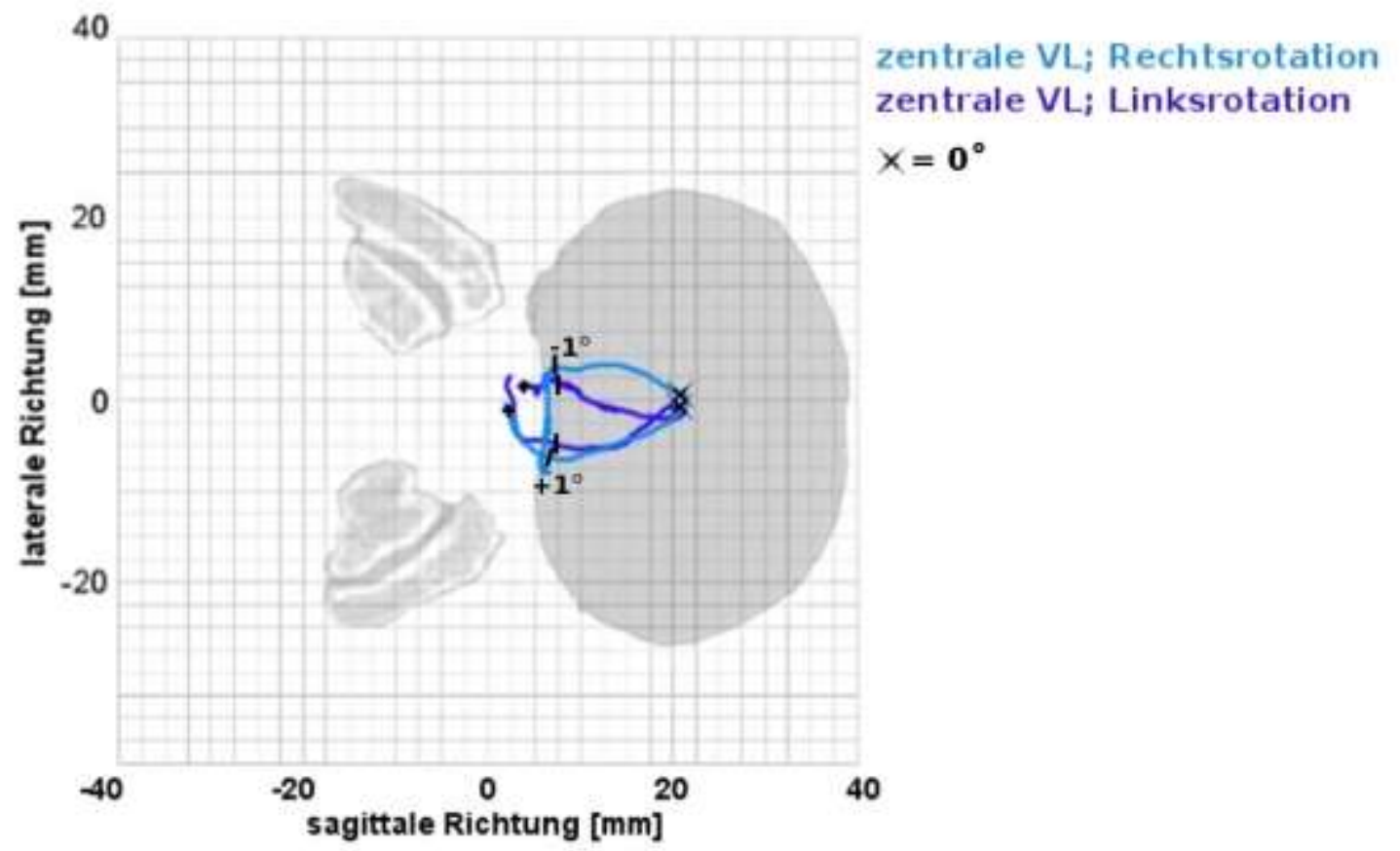

Abbildung 23: Beispiel einer Rastpolkurve (blau und türkis) unter Axialrotation. Die Konturen des Wirbelkörpers und der Wirbelgelenke aus kranialer Sicht sind grau eingezeichnet. 


\subsubsection{Ausrichtung der momentanen Schraubachsen}

Die Darstellung der Ausrichtung der momentanen Schraubachsen geschieht stets in Relation zur Achse des einwirkenden Drehmoments, d.h. es werden die Winkel (in Grad) angegeben, die die momentane Schraubachse von der Referenzachse (Drehmomentachse) abweicht. Um die genaue Ausrichtung der momentanen Schraubachse zu definieren, wird die Abweichung in jeweils zwei Richtungen beschrieben. Für das Experiment „Axialrotation“ bedeutet dies, dass eine momentane Schraubachse, die parallel zur z-Achse verläuft, einen Abweichungswinkel von $0^{\circ}$ aufweist. Eine geneigte Schraubachse kann nun einerseits in der sagittalen Ebene nach ventral oder dorsal abweichen, andererseits in der Frontalebene nach rechts oder links. In der Versuchsreihe "Ventralflexion-Dorsalextension“ wird ein Drehmoment in y-Richtung angelegt, so dass nun die Schraubachsen parallel zur y-Achse einen Neigungswinkel von $0^{\circ}$ haben. Abweichungen können nach kranial oder kaudal bzw. nach ventral oder dorsal auftreten. Für die Experimente "Lateralflexion“ zeigt sich entsprechend eine Abweichung von $0^{\circ}$ bei Schraubachsen parallel zur $x$-Achse. Es sind Neigungen nach kranial oder kaudal sowie nach rechts oder links möglich.

Die errechneten Abweichungswinkel werden am Ende der entsprechenden Abschnitte in zwei Tabellen zusammenfassend dargestellt. Dabei beziehen sich die beiden Tabellen auf jeweils eine der beiden Richtungen, in die die momentane Schraubachse von der Drehmomentachse abweichen kann. In der Versuchsreihe „Axialrotation“ werden beispielsweise in der ersten Tabelle die Neigungswinkel in sagittaler Richtung (ventraldorsal), in der zweiten Tabelle die Winkel in lateraler Richtung (links-rechts) aufgeführt. Das Vorzeichen beschreibt dabei die "Seite“, zu der die Abweichung stattfindet. Positive Vorzeichen bedeuten, in Abhängigkeit von der Richtung, eine Neigung nach ventral, links oder kranial, negative Vorzeichen entsprechend nach dorsal, rechts oder kaudal. Die Abkürzung Z.n. in der Tabellenbeschriftung bedeutet „Zustand nach“.

Die Tabelle (Tabelle 3) unterscheidet zunächst die drei Resektionszustände „intaktes Segment", „Segment nach Resektion des rechten Gelenks“ und „BandscheibenBänder-Präparat" in der ersten Spalte. In der zweiten Spalte wird für jeden Segmentzustand zwischen zentraler, ventraler und dorsaler Vorlastposition differenziert. Das durch das Drehmoment bedingte Rotationsausmaß wird in den Spalten drei bis fünf in drei Rotationszustände gegliedert. Liegt ein nichtrotiertes Segment vor, ist der Drehwinkel $\alpha=0^{\circ}$ (Spalte 3), die Rotationsmaxima werden in den Spalten vier und fünf dargestellt.

Bei den Angaben der Winkel handelt es sich um Mittelwerte aus allen gemessenen Präparaten unter denselben Versuchsbedingungen, die Standardabweichung ist jeweils in Klammern dargestellt.

Beispiele (bezogen auf Tabelle 3):

Bei intaktem Segment und ventraler Vorlast ist die Schraubachse bei nichtrotiertem Segment $\left(\alpha=0^{\circ}\right)$ um $7^{\circ}$ nach rechts geneigt. Nach der Resektion des rechten Wirbelbogengelenks ist die Achse bei zentraler Vorlast und im rechtsrotierten Zustand um $10^{\circ}$ 
nach links geneigt. Beim Bandscheiben-Bänder-Präparat und dorsaler Vorlast ist die Achse im linksrotierten Zustand um $4^{\circ}$ nach links geneigt.

Tabelle 3: Beispieltabelle mit willkürlich gewählten Werten: Abweichung nach lateral der momentanen Schraubachse in Grad von einer vertikalen Achse. Unterschieden werden 1. Segmentzustand: intaktes Segment, nach Resektion des rechten Gelenks, Bandscheiben-Bänder-Präparat; 2. Vorlastposition: zentral, ventral, dorsal; 3. Rotationsausmaß: keine Rotation $\left(\alpha=0^{\circ}\right)$, maximale Rechts- bzw. Linksrotation; Die Standardabweichung ist in Klammern angegeben.

\begin{tabular}{|c|c|c|c|c|c|c|c|}
\hline Segmentzustand & VL-Position & \multicolumn{2}{|c|}{$\alpha=0^{\circ}$} & \multicolumn{2}{|c|}{ Max rechtsrotiert } & \multicolumn{2}{|c|}{ Max linksrotiert } \\
\hline \multirow{3}{*}{ intaktes Segment } & zentral & 3 & (5) & 5 & (4) & 6 & (3) \\
\hline & ventral & -7 & (6) & -9 & (8) & -9 & (4) \\
\hline & dorsal & 9 & (2) & 8 & (2) & 8 & (3) \\
\hline \multirow{3}{*}{$\begin{array}{l}\text { nach Resektion des } \\
\text { rechten Gelenks }\end{array}$} & zentral & 4 & (2) & 10 & (7) & -2 & (7) \\
\hline & ventral & -6 & (4) & -15 & (7) & -8 & (2) \\
\hline & dorsal & 6 & (7) & 5 & (7) & 11 & (11) \\
\hline \multirow{3}{*}{$\begin{array}{c}\text { Bandscheiben-Bänder- } \\
\text { Präparat }\end{array}$} & zentral & 2 & (2) & 4 & (5) & 5 & (3) \\
\hline & ventral & -7 & (6) & 1 & (6) & 2 & (5) \\
\hline & dorsal & 8 & (2) & 3 & (4) & 4 & (1) \\
\hline
\end{tabular}

\subsubsection{Drehwinkel-Drehmoment-Kennlinie und Bewegungsausmaß}

Trägt man das Drehmoment T gegen den absoluten Drehwinkel $\alpha$ auf, erhält man die Drehmoment-Drehwinkel-Kennlinie, die in dieser Abbildung angezeigt wird. Weiterhin lässt sich das Bewegungsausmaß (range of motion, ROM) des Segments sowie die Größe der Hysterese feststellen (Abbildung 24).

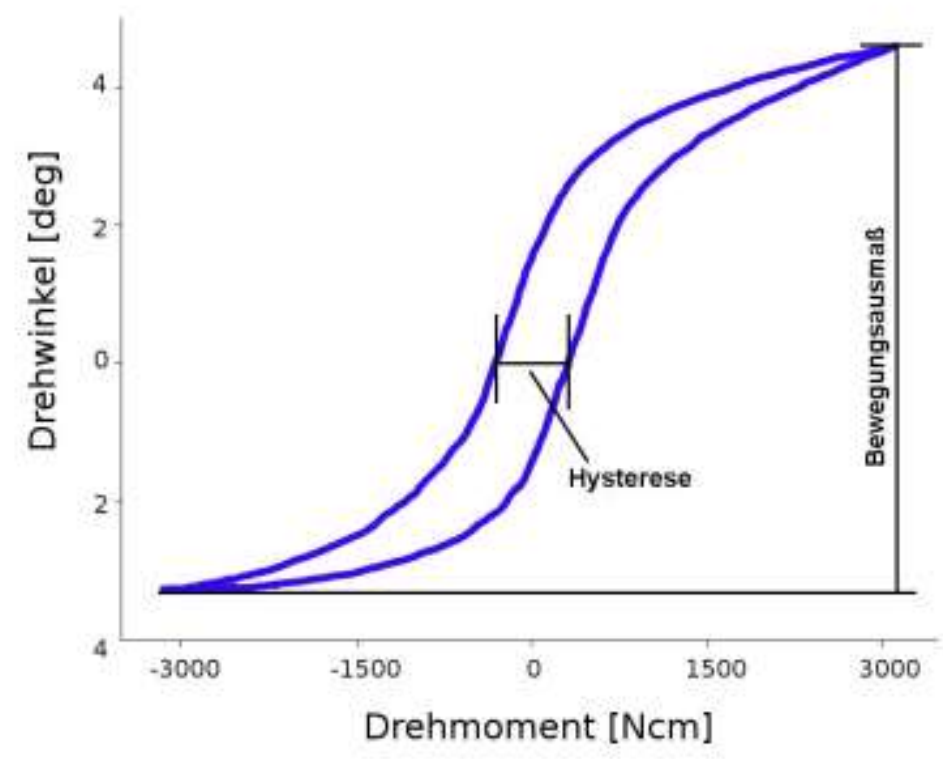

Abbildung 24: Beispiel einer Drehwinkel-Drehmoment-Kennlinie. Das Bewegungsausmaß und die Hysterese sind eingezeichnet. 
Zur vergleichenden Darstellung des ROM erfolgte die Berechnung eines Mittelwertes aus den Daten aller Segmente. Die prozentualen Veränderungen wurden gebildet, indem zunächst die Relation innerhalb eines Segments ermittelt und aus den daraus gewonnenen Werten der Mittelwert errechnet wurde.

\subsubsection{Steifigkeitsverhalten}

Durch die Ermittlung der reziproken Steigung der Drehmoment-Drehwinkel-Kennlinie erhält man die Steifigkeitskurve, die im Steifigkeitsdiagramm dargestellt wird. Sie entspricht der momentanen Steifigkeit $\mathrm{T}^{\prime}(\alpha)=\mathrm{dT} / \mathrm{d} \alpha$ in Abhängigkeit vom Drehwinkel. Im Umkehrpunkt der Kurve findet sich stets die minimale Steifigkeit, die zur Erstellung von Diagrammen herangezogen werden kann (Abbildung 25).

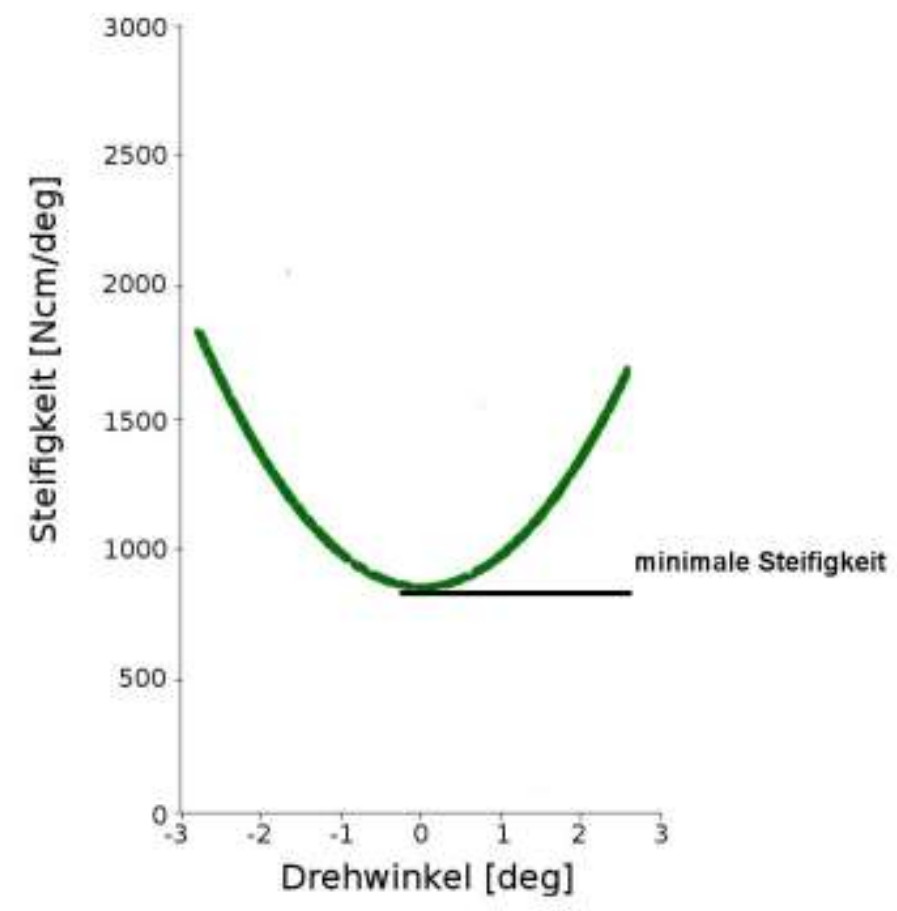

Abbildung 25: Beispiel einer Steifigkeitskurve. Der Punkt der minimalen Steifigkeit ist markiert.

Das Vorgehen zur Erstellung der Diagramme und Tabellen wurde bereits im vorherigen Abschnitt 2.4.3 beschrieben.

\subsection{Aufbau der Studie}

In der Studie wurden zur Untersuchung der Funktion der Wirbelbogengelenke mehrere Variablen gewählt: Resektionszustand, Rotationsrichtung und Größe und Position der Vorlast. Die Durchführung der Studie umfasste sieben Punkte: 
1. Messung an einer Präzisionsschraube (Abbildung 22) zur Kalibrierung und Ausrichtung der Messapparatur

2. Messung an einem Bandscheibenmodell (Abbildung 22)

3. Messungen am intakten Segment

- Axialrotation $(3240 \mathrm{Ncm})$

- Ventralflexion-Dorsalextension (580 Ncm-1015 Ncm)

- Lateralflexion (1450 Ncm)

4. Resektion des rechten Wirbelbogengelenks

5. erneute Messungen

- Axialrotation (2160 Ncm-3240 Ncm)

- Ventralflexion-Dorsalextension (580 Ncm-1015 Ncm)

- Lateralflexion (725 Ncm-1450 Ncm)

6. Resektion des linken Wirbelbogengelenks

7. Messungen am Bandscheiben-Bänder-Präparat

- Axialrotation (2160 Ncm-3240 Ncm)

- Ventralflexion-Dorsalextension (580 Ncm-870 Ncm)

- Lateralflexion (1160 Ncm-1450 Ncm)

Um einer Beschädigung der Präparate zu vorzubeugen, wird die Höhe des Drehmoments an den Zustand des Präparats, die Rotationsrichtung und den Resektionsstand angepasst.

Die einzelnen Messungen folgten einem festen Schema:

1. Einschwingen der Messapparatur von ca. 30 Minuten

2. Messung mit Vorlasten von 0 N, $200 \mathrm{~N}$ und $400 \mathrm{~N}$ im Zentrum (Position 0)

3. Verschieben einer konstanten Vorlast von $200 \mathrm{~N}$ nach:

- ventral und dorsal: Positionen je $2,5 \mathrm{~mm}, 5 \mathrm{~mm}, 10 \mathrm{~mm}, 15 \mathrm{~mm}, 20 \mathrm{~mm}$, $40 \mathrm{~mm}, 60 \mathrm{~mm}$

- rechts und links: Positionen je $5 \mathrm{~mm}, 10 \mathrm{~mm}, 15 \mathrm{~mm}, 20 \mathrm{~mm}, 40 \mathrm{~mm}, 60 \mathrm{~mm}$

4. Datenerfassung zu jeder Messung 


\section{Ergebnisse}

In diesem Kapitel werden die zusammengefassten Messergebnisse der Versuchsreihen präsentiert. Zunächst ist die Überprüfung der Validität der Daten dargestellt. Es folgt die Präsentation der Daten der Messungen in Axialrotation, Flexion-Extension und Lateralflexion. Zur besseren Übersicht werden nicht alle gemessenen Daten gezeigt, sondern eine Beschränkung auf repräsentative Ergebnisse vorgenommen. Sofern nicht aus dem Text ersichtlich, handelt es sich bei den Zahlenangaben um Durchschnittswerte.

\section{1 Überprüfung der Messapparatur}

Die Überprüfung der Messapparatur erfolgte in drei Schritten. Zunächst wurde eine Präzisionsschraube mit bekannten Fertigungseigenschaften gemessen und die Ergebnisse mit den Erwartungswerten verglichen. Es folgte der Einsatz eines Bandscheibenmodells ohne und mit Installation von Modellen der Zwischenwirbelgelenke. Die Ergebnisse dieser Messungen dienten wiederum dem Vergleich mit den Erwartungswerten.

\subsubsection{Präzisionsschraube}

Die Messungen ergaben eine differentielle Schraubsteigung von 0,0062 $\mathrm{mm} / \mathrm{deg}$ und eine in z-Richtung weisende Schraubachse. Die momentanen Schraubachsen entfernten sich um maximal $2 \mathrm{~mm}$ vom Schraubenzentrum, verhielten sich somit nahezu zentral und ortsfest (Abbildung 26). Die gemessenen und aufgrund der Fertigungsmerkmale erwarteten Ergebnisse stimmten weitgehend überein (Tabelle 4).

Tabelle 4: Vergleich der erwarteten und gemessenen Ergebnisse der Messung der Präzisionsschraube

\begin{tabular}{|l|l|l|}
\hline & gemessen & erwartet \\
\hline Schraubsteigung & $\approx 0,0062 \mathrm{~mm} /{ }^{\circ}$ & $0,005 \mathrm{~mm} /{ }^{\circ}$ \\
\hline Richtung der Schraubachse & z-Richtung & z-Richtung \\
\hline Rastpolkurve & nahezu zentral & zentral \\
\hline Wanderung der Schraubachse & nahezu ortsfest & ortsfest \\
\hline
\end{tabular}

\subsubsection{Bandscheibenmodell}

Die Versuche mit dem Bandscheibenmodell fanden unter einem manuell angebrachten axialen, möglichst konstanten Drehmoment statt, die Vorlast betrug $20 \mathrm{~N}$, die 
Vorlastpositionen $0 \mathrm{~mm}, 40 \mathrm{~mm}$ ventral sowie $20 \mathrm{~mm}$ dorsal. Bei zentraler Vorlastposition konnte keine Schraubsteigung festgestellt werden. Die Schraubachse verlief parallel zur z-Achse und wanderte im Rotationsverlauf im maximalen Abstand von $3 \mathrm{~mm}$ um den Nullpunkt herum (Abbildung 26). Die Drehwinkel-DrehmomentKennlinie zeigte eine hockeyschlägerartige Form und eine Hysterese. Die Steifigkeit betrug $17 \mathrm{Ncm} / \mathrm{deg}$.

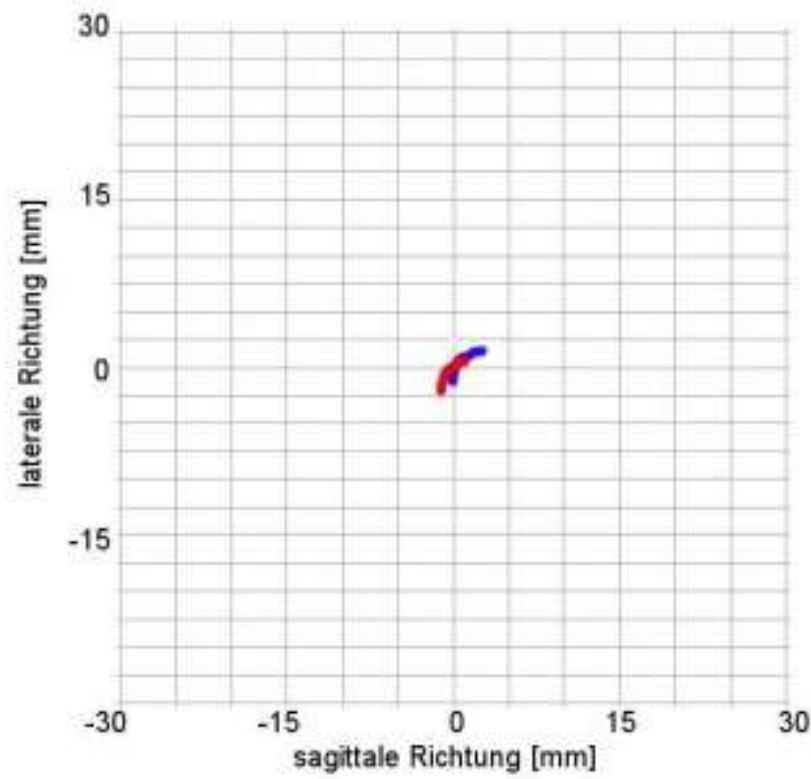

Abbildung 26: Rastpolkurven von Präzisionsschraube (rot) und Bandscheibenmodell (blau) bei zentraler Vorlast

Die Messresultate nach Verschiebung der Vorlast nach ventral bzw. dorsal entsprachen denen bei zentraler Vorlast. Lediglich die Schraubachsen verkippten dezent nach ventral bzw. dorsal.

Nach Einsatz der Modelle der Zwischenwirbelgelenke war in Neutralstellung (Drehwinkel $\alpha=0^{\circ}$ ) keine Schraubsteigung vorhanden. In den Rotationsmaxima betrug sie jeweils etwa $0,02 \mathrm{~mm} / \mathrm{deg}$. Die Schraubachsen lagen in Neutralstellung parallel zur z-Achse im Zentrum des Puffers. Im Rotationsverlauf wanderten sie bis zu einer Länge von $12 \mathrm{~mm}$ in Richtung eines Gelenkmodells: während der Linksdrehung zum linken Gelenkmodell, während der Rechtsdrehung zum rechten. Bei Drehwinkeln über $7^{\circ}$ schwenkten die Achsen zügig nach dorsal und medial (Abbildung 27). Die Schraubachsen neigten sich im Rotationsmaximum nach dorso-lateral. Die DrehwinkelDrehmoment-Kennlinie war weiterhin hockeyschlägerartig geformt und wies eine Hysterese auf. Die minimale Steifigkeit lag bei 20 Ncm/deg.

Durch Erhöhung oder Verschiebung der Vorlast nach dorsal wurde die laterale Ausdehnung der Rastpolkurve ausladender. Bei ventraler Vorlast erhielt man ähnliche Ergebnisse wie ohne die Gelenkmodelle. 


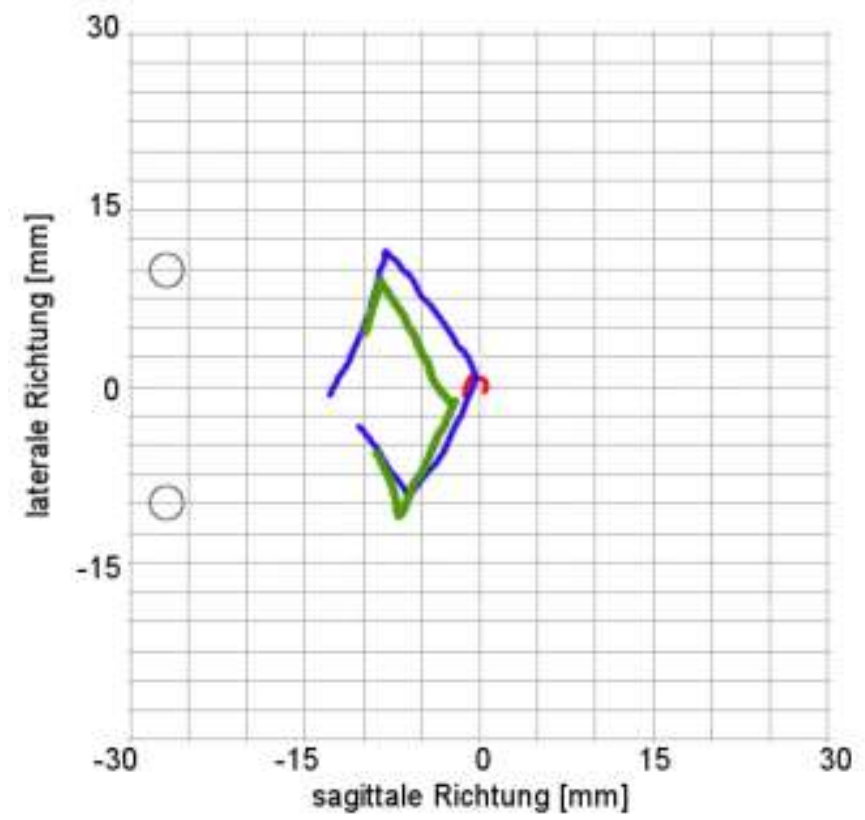

Abbildung 27: Rastpolkurven des Bandscheiben-Gelenk-Modells bei zentraler (grün), $40 \mathrm{~mm}$ ventraler (rot) und $20 \mathrm{~mm}$ dorsaler (blau) Vorlastposition; die Kreise markieren die Position der Gelenkmodelle. Eine Wanderung der Rastpolkurve in Richtung der Gelenke wurde bei zentraler und dorsaler Vorlastposition sichtbar.

\subsection{Axialrotation}

In diesem Abschnitt werden die Ergebnisse des Experiments „Axialrotation“ dargestellt. Zunächst erfolgt die Präsentation der Rastpolkurven, also der Position der momentanen Schraubachsen, unter verschiedenen Versuchsbedingungen (Variation von Resektionszustand und Vorlastposition). Anschließend werden die Ausrichtung der Schraubachsen (Abweichung von einer vertikalen Achse), das Bewegungsausmaß und das Steifigkeitsverhalten besprochen.

\subsubsection{Rastpolkurven}

\subsubsection{Sagittale Verschiebung der Vorlast}

\section{$\underline{\text { Intaktes Segment }}$}

Bei zentraler Vorlastposition (im Wirbelkanal) war die Rastpolkurve annähernd dreiecksförmig. Sie begann bei maximaler Linksdrehung in der linken Hälfte des Spinalkanals und wanderte dann nach rechts und leicht nach ventral. Etwa am dorsalen Rand der Bandscheibe schwenkten die Schraubachsen in Richtung Bandscheibenmitte. Dort befand sich die Spitze des Dreiecks sowie der Scheitelpunkt der Rotation $\left(\alpha=0^{\circ}\right)$. Von dort führte die Kurve zunächst in Richtung des linken Zwischenwirbelgelenks, um wiederum am Bandscheibenrand zur gegenüberliegenden rechten Seite $a b$ zu knicken. Am Ende der Rechtsdrehung verlief die Kurve relativ parallel zum Bandscheibenrand. Zu Beginn der Bewegungsumkehr kam es zu einem leichten Versatz nach links-dorsal, ansonsten entsprach die Linksdrehung der Rechtsdrehung. Während eines Halbzyklus wanderten die Schraubachsen am Beispiel 
des Präparats B um maximal $20 \mathrm{~mm}$ in sagittaler sowie $10 \mathrm{~mm}$ in lateraler Richtung (Abbildung 28).

Durch Verlagerung der Vorlast um $60 \mathrm{~mm}$ nach ventral wurde die Dreiecksform der Rastpolkurve deutlicher. Die Ecken erschienen spitzer, die Basis breiter. Die Kurve bei Rechtsdrehung begann nun auf Höhe des rechten Gelenks und machte lediglich einen kurzen Schlenker nach lateral, bevor sie zur Spitze weiterführte. Diese lag nun eher am dorsalen Rand der Bandscheibe, war also um bis zu $10 \mathrm{~mm}$ nach dorsal versetzt. Von dort verlief die Kurve zum linken Gelenk und endete mit einer kurzen Strecke in medialer Richtung. Die Linksdrehung erfolgte umgekehrt in ähnlicher Weise. Dieser Kurvenverlauf war deutlich im Präparat $C$ zu sehen. Hier betrug die maximale ventrodorsale Ausdehnung der Kurve nun noch $16 \mathrm{~mm}$, die laterale war auf $20 \mathrm{~mm}$ vergrößert.

Nach 60 mm Dorsalverschiebung der Vorlast erschien die Rastpolkurve hufeisenförmig bis rund. Im Präparat $C$ verlief sie bei Rechtsdrehung beginnend am rechten dorsalen Rand der Bandscheibe im Bogen durch dessen dorsale Hälfte bis zum linken Rand und bei Gegendrehung parallel wieder zurück. Bei einer Gesamtrotation von $4^{\circ}$ verweilten die Schraubachsen während einer Drehung um jeweils $1^{\circ}$ am dorsalen Rand und wanderten während des nächsten Grades zum Scheitelpunkt des „Hufeisens“. Die Kurve lag insgesamt etwa $2 \mathrm{~mm}$ weiter dorsal als bei zentraler Vorlast. Im Verlauf dieser Bewegung verlagerten sich die Schraubachsen um jeweils $16 \mathrm{~mm}$ nach ventral und rechts (Abbildung 28).

Bei den anderen Präparaten wirkte die Kurve insgesamt gedrungener als bei zentraler Vorlast. 


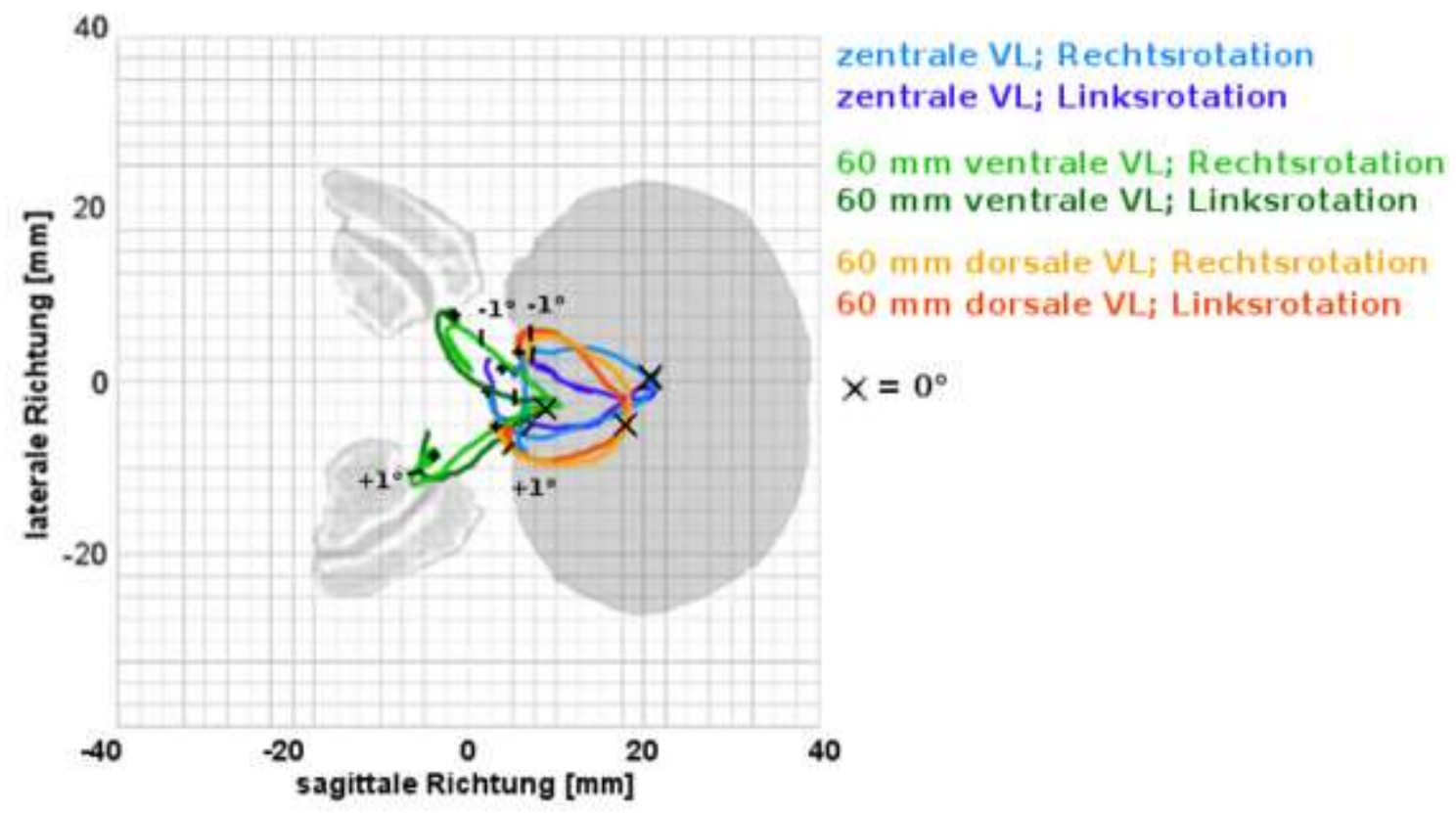

Abbildung 28: Rastpolkurven des intakten Präparats $\mathrm{C}$ bei zentraler, ventraler und dorsaler Vorlast $(200 \mathrm{~N})$. Auffallend war die spitze Form und dorsale Position bei ventraler Vorlast sowie die runderer Form bei dorsaler Vorlast. ; Axialrotation; die Lage des Wirbels ist eingeblendet; + markiert den Bewegungsbeginn; die Markierungen mit Zahlen beschreiben das Rotationsausmaß.

\section{$\underline{\text { Segment nach Resektion des rechten Zwischenwirbelgelenks }}$}

Da die Präparate nach jedem Resektionsschritt instabiler und somit anfälliger für Beschädigungen waren, wurden das Drehmoment und die maximalen Vorlastpositionen gegebenenfalls herabgesetzt. Das Präparat E ist im Laufe der Messungen beschädigt worden, so dass die gemessenen Daten nur für einen Teil der Ergebnisse verwendet werden konnten.

Bei zentraler Vorlastposition verlief die erste Hälfte der Linksrotation in etwa wie die des intakten Segments. Jedoch war die Rastpolkurve um $6 \mathrm{~mm}$ nach links und $4 \mathrm{~mm}$ nach ventral verlagert. Am Präparat $C$ erkannte man beispielhaft, dass, im Gegensatz zum intakten Segment, bei Drehwinkeln von $\alpha>0^{\circ}$ die Kurve im Bogen nach links und dorsal weiterführte. Die Rechtsrotation erfolgte weitgehend parallel (Abbildung 29). Mit einer maximalen Ausdehnung von $18 \mathrm{~mm}$ in sagittaler und $15 \mathrm{~mm}$ in lateraler Richtung war die Rastpolkurve kürzer und breiter als im intakten Segment.

Durch Ventralverlagerung der Vorlast um $60 \mathrm{~mm}$ begann die Rastpolkurve in maximaler Rechtsrotation in der dorsalen Hälfte der Bandscheibe und im mittleren Drittel in lateraler Ausbreitung. Sie wanderte langsam um $7 \mathrm{~mm}$ nach ventral und bog im spitzen Winkel über eine Strecke von $20 \mathrm{~mm}$ zum verbliebenen Gelenk hin ab. Wie auch beim intakten Segment kam es zu einer Dorsalverlagerung der Kurve um $10 \mathrm{~mm}$. Der Verlauf in der rechten Hälfte der Bandscheibe fehlte nach der Resektion jedoch völlig. Der antero-posteriore Durchmesser war mit $16 \mathrm{~mm}$ deutlich kleiner als vor der Resektion.

Nach Verschiebung der Vorlast um $60 \mathrm{~mm}$ nach dorsal verlief die Rastpolkurve während der Rechtsdrehung vom linken Drittel der Bandscheibe zum linken 
Zwischenwirbelgelenk. Das Präparat C zeigte zusätzlich zu Beginn der Rotation eine Bewegung nach ventral-medial. Auch nach der Resektion lag die Kurve nun 2 mm weiter dorsal als bei zentraler Vorlastposition (Abbildung 29). Im Vergleich zum intakten Segment fiel auch hier die links-laterale Lage (8 $\mathrm{mm}$ vom Median) der Rastpolkurve sowie das Fehlen der rechten Komponente auf.

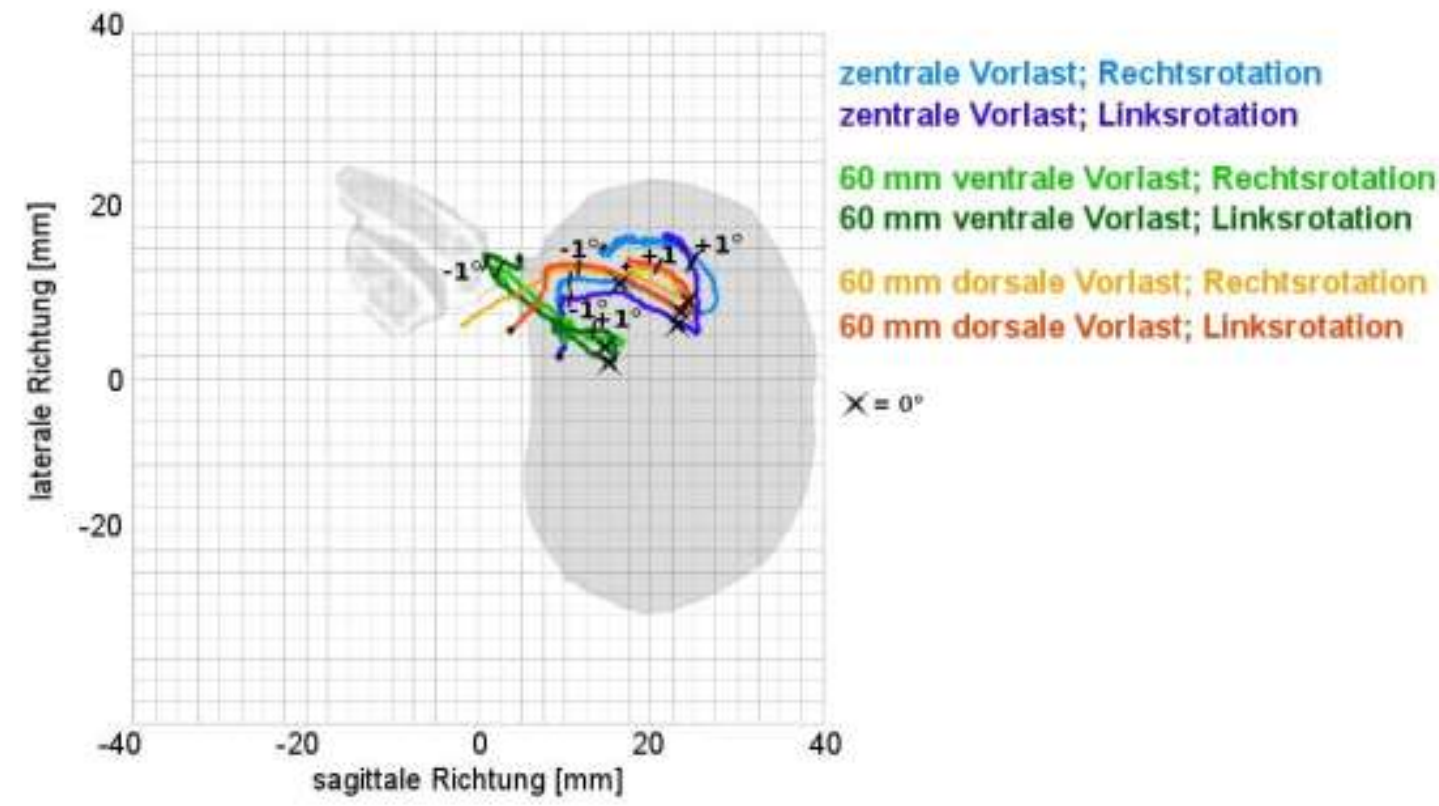

Abbildung 29: Rastpolkurven des Präparats C nach Resektion des rechten Zwischenwirbelgelenks bei zentraler, ventraler und dorsaler Vorlast $(200 \mathrm{~N})$. Die Kurven waren deutlich nach links verschoben. Axialrotation; die Lage des Wirbels ist eingeblendet; + markiert den Bewegungsbeginn; die Markierungen mit Zahlen beschreiben das Rotationsausmaß.

\section{Bandscheiben-Bänder-Präparat}

Die Rastpolkurven verliefen mit zentral positionierter Vorlast nahe der Bandscheibenmitte. Am Beispiel des Präparats C waren sie besonders im sagittalen Ausmaß deutlich kompakter als beim intakten Segment und lagen etwa $15 \mathrm{~mm}$ weiter ventral. Bei Drehwinkeln $<0^{\circ}$ wanderten die Rastpole in die rechte Hälfte der Bandscheibe, bei Winkeln $>0^{\circ}$ in die linke (Abbildung 30). Die Abweichung in lateraler Richtung betrug jeweils maximal $6 \mathrm{~mm}$ vom Kurvenzentrum, in sagittaler Richtung $3 \mathrm{~mm}$. Der Vergleich der Links- mit der Rechtsdrehung zeigte bei kleinen Drehwinkeln bis ca. $2^{\circ}$ eine Spiegelung an einer transversalen Achse: In der Linksdrehung befanden sich die Schraubachsen bei geringen negativen Winkeln ventral, bei positiven Drehwinkeln dorsal des Kurvenmittelpunkts. Im Verlauf der Rechtsrotation dagegen lagen sie zunächst ventral (positive Winkel) und dann dorsal (negative Winkel).

Nach Verlagerung der Vorlast nach ventral $(40 \mathrm{~mm})$ blieb die Position der Rastpolkurve im Gegensatz zum unresezierten Zustand weitgehend erhalten. Mit einem sagittalen bzw. Iateralen Durchmesser von jeweils $10 \mathrm{~mm}$ war sie schmaler als bei zentraler 
Vorlast. Links- und Rechtsrotation verliefen nun parallel. Im Vergleich zum intakten Segment war die Kurve deutlich komprimiert und knapp 20 mm weiter ventral gelegen.

Die Rastpolkurven bei $20 \mathrm{~mm}$ dorsaler Vorlastposition entsprachen in Form und Position ungefähr denen bei zentraler Vorlast (Abbildung 30). Auch die dort beschriebene Spiegelung wurde deutlich. Die Ausdehnung war mit Abweichungen vom Mittelpunkt der Kurve von je $5 \mathrm{~mm}$ besonders in lateraler Richtung verkleinert. Verglichen mit dem intakten Segment fehlte die dezente Dorsalverschiebung der Kurve. Insgesamt lag sie etwa $13 \mathrm{~mm}$ weiter ventral.

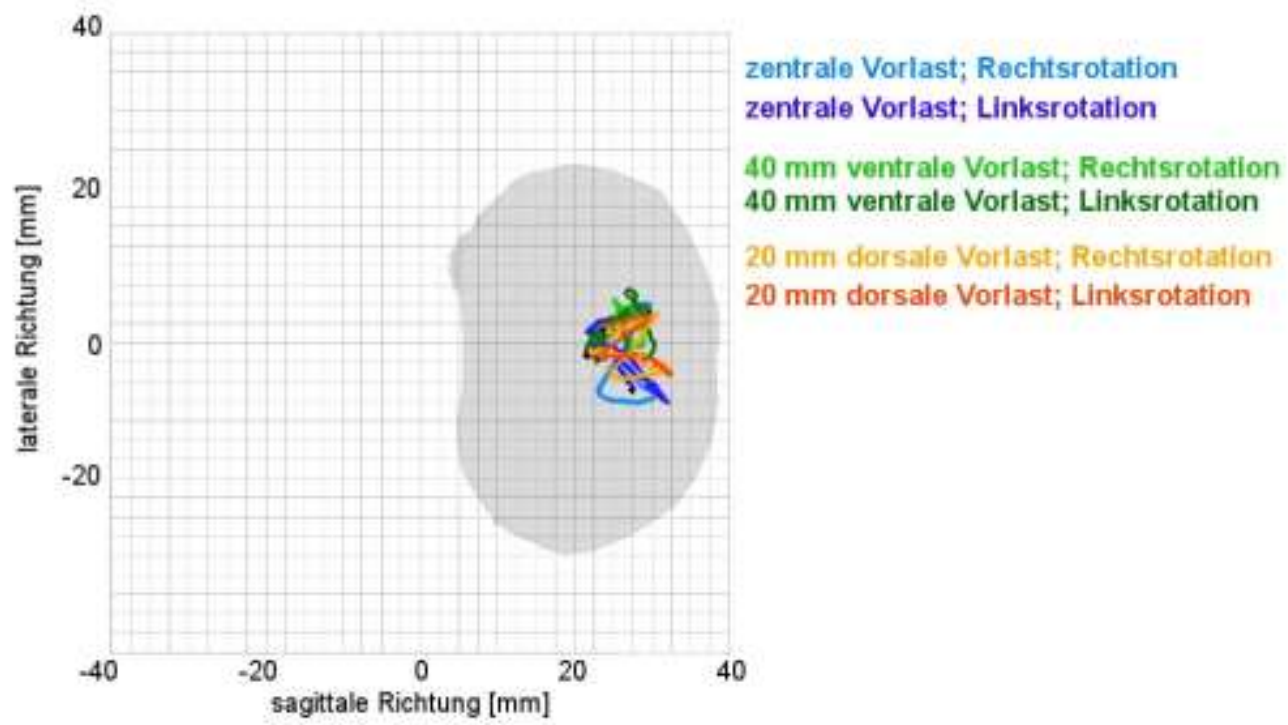

Abbildung 30: Rastpolkurven des Bandscheiben-Bänder-Präparats $C$ bei zentraler, ventraler und dorsaler Vorlast (200 N). Die Kurven befanden sich unabhängig von der Vorlastposition und relativ ortsfest in der Bandscheibe. Axialrotation; die Lage des Wirbels ist eingeblendet; + markiert den Bewegungsbeginn.

Zur Übersicht zeigt Abbildung 31 die Rastpolkurven aller Resektionsstufen bei ventraler Vorlastposition. Beim Vergleich der Kurven fällt auf, dass der Verlauf nach der Resektion des rechten Zwischenwirbelgelenks bei negativen Drehwinkeln (rechtsrotiertes Segment) parallel zu der Kurve des intakten Segments ist, während positive Drehwinkel zu einem ortsfesten Verlauf führen. 


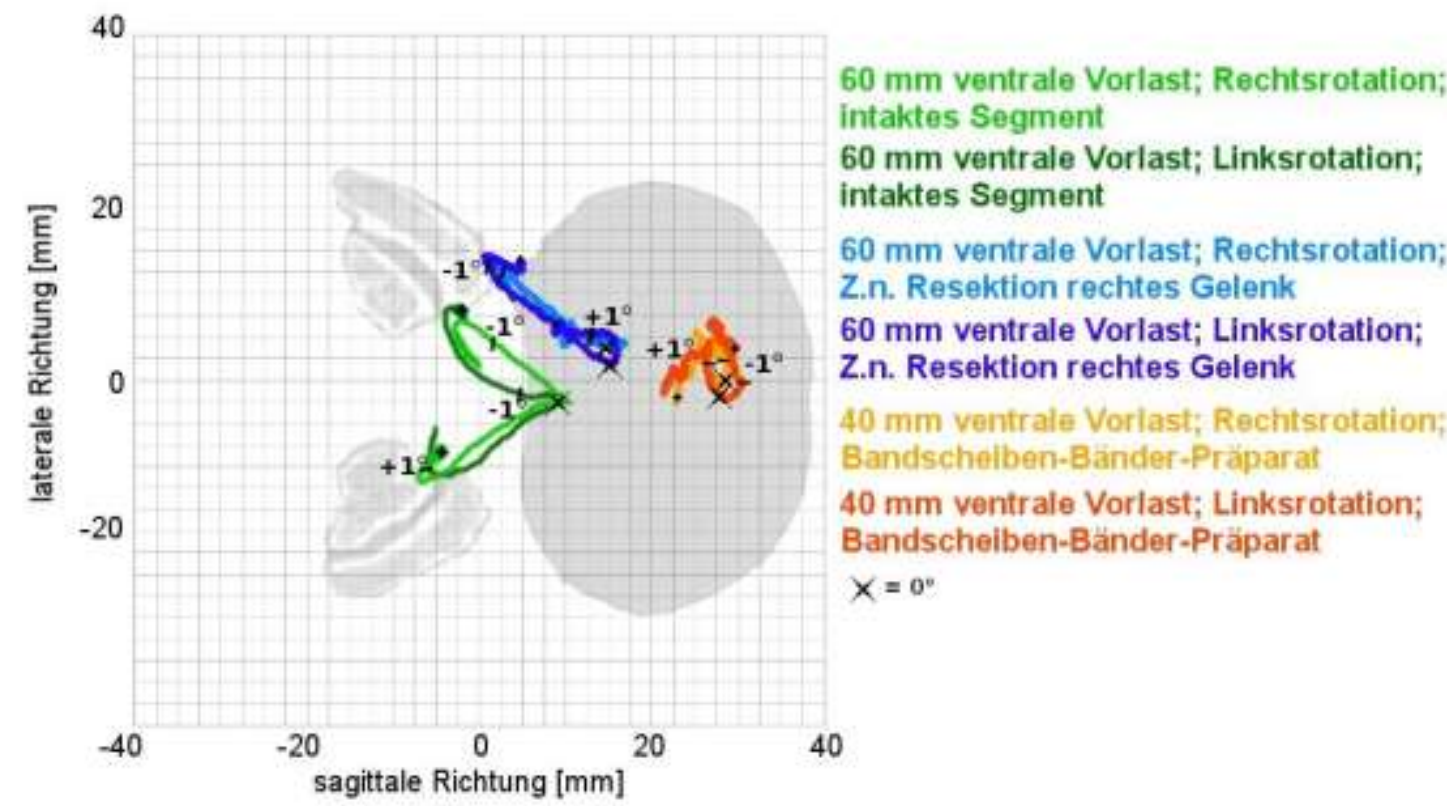

Abbildung 31: Vergleich der Rastpolkurven bei ventraler Vorlast (200 N) bei unterschiedlichen Resektionszuständen. Nach der Resektion des rechten Gelenks führten besonders negative Rotationswinkel zu qualitativen Unterschieden im Kurvenverlauf. Axialrotation; die Lage des Wirbels ist eingeblendet; + markiert den Bewegungsbeginn; die Markierungen mit Zahlen beschreiben das Rotationsausmaß.

\subsubsection{Laterale Verschiebung der Vorlast}

\section{Intaktes Segment}

Die Rastpolkurve verlief in einer Schleife im dorsalen Bereich der Bandscheibe und verlängerte sich dann in Richtung des Gelenks, auf dessen Seite die Vorlast angebracht war, d.h. bei 40 mm links-lateraler Vorlast in Richtung des linken Gelenks. Exemplarisch sei die Kurve des Präparats C bei rechtsseitiger Vorlast $(40 \mathrm{~mm})$ beschrieben (Abbildung 32): Im maximal rechtsrotierten Zustand begann die Kurve am dorsalen Rand der Bandscheibe links der Medianlinie. Zu Beginn der Linksrotation wanderten die Schraubachsen langsam in Richtung Bandscheibenmitte. Dort beschrieben sie eine spitze Kurve und verliefen zügig in einem lateral führenden Bogen zum rechten Gelenk. Ein langsamer kleiner Schwenk zur Spinalkanalmitte beendete die Drehung. Die Rechtsrotation lief ähnlich in umgekehrter Richtung ab. Die Kurve war lediglich am Bandscheibenzentrum runder und führte am Ende der Bewegung parallel zum Bandscheibenrand nach rechts. Dadurch kam es beim Wechsel von Rechts- zu Linksdrehung zu einem Abbrechen und Springen der Rastpolkurve. Im Vergleich zur zentralen Vorlast war die Kurve also dezent (ca. $2 \mathrm{~mm}$ ) in Vorlastrichtung verlagert, der laterale Durchmesser blieb jedoch unverändert. Die ventro-dorsale Ausdehnung vergrößerte sich auf $23 \mathrm{~mm}$. 


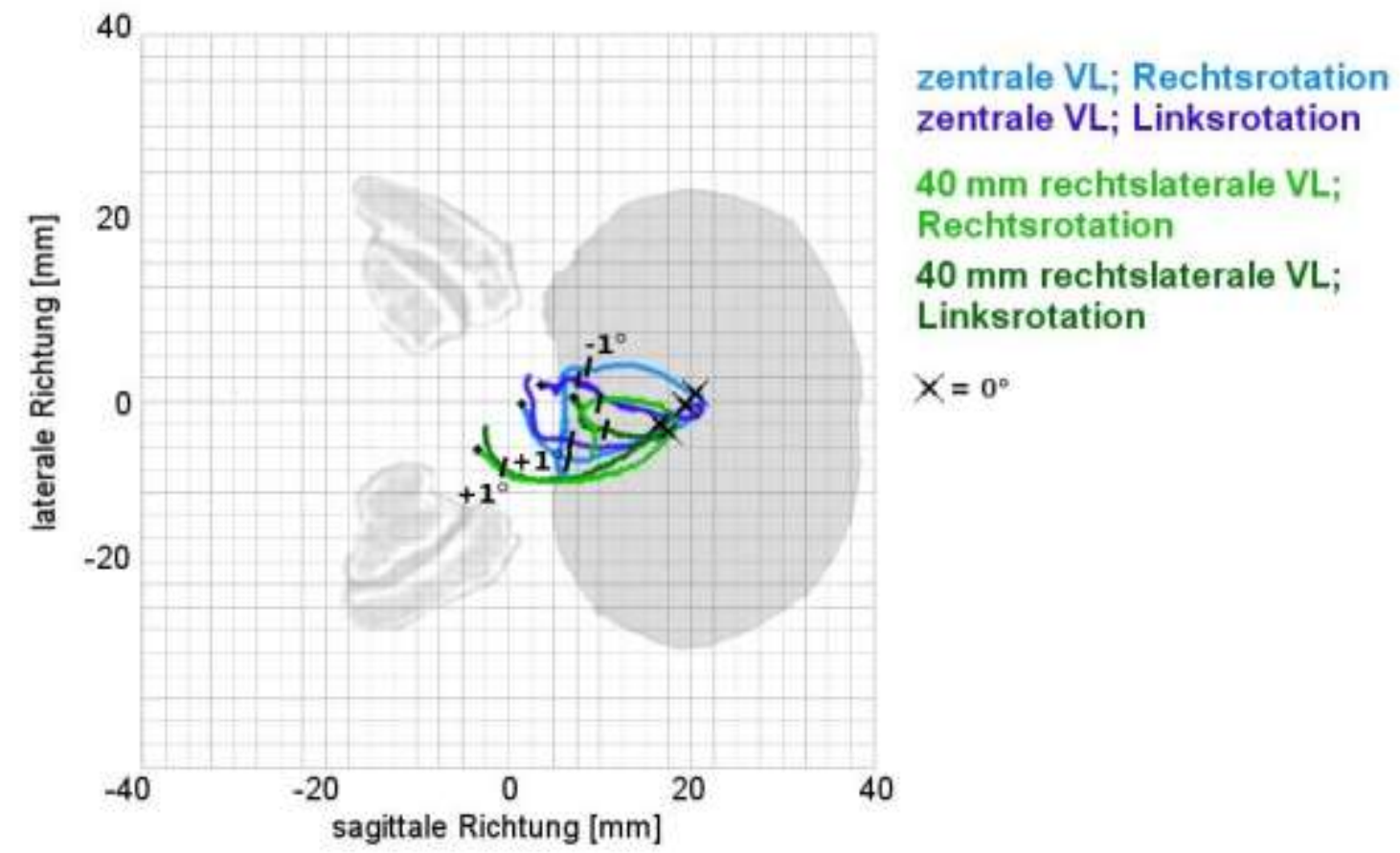

Abbildung 32: Rastpolkurven des intakten Präparats C bei zentraler und rechts-lateraler Vorlast (200 N). Eine laterale Vorlast führte zur Verlagerung der Kurve nach lateral und dorsal. Axialrotation; die Lage des Wirbels ist eingeblendet; + markiert den Bewegungsbeginn; die Markierungen mit Zahlen beschreiben das Rotationsausmaß.

\section{Segment nach Resektion des rechten Zwischenwirbelgelenks}

Die Rastpolkurven des Präparats C waren im Verlauf ähnlich wie bei zentraler Vorlastposition. Die Kurve bei $40 \mathrm{~mm}$ rechts-lateral platzierter Vorlast erschien jedoch im anterior-posterioren Ausmaß gedrungener, bei $40 \mathrm{~mm}$ links-lateraler Vorlastposition leicht nach dorsal verschoben (Abbildung 33).

Beide Kurven - sowohl bei linker als auch rechter Vorlastposition - lagen in der linken Wirbelhälfte, die der linksseitigen Vorlast jedoch weiter links als die der rechtsseitigen. Es konnte also im Gegensatz zum intakten Segment auch durch rechtsseitige Vorlast keine Verschiebung der Kurve in die rechte Bandscheibenhälfte erreicht werden. Die Wanderung der Schraubachsen zwischen linker Bandscheibenhälfte (max. linksrotiert) und Gelenk (max. rechtsrotiert) war bei linker Vorlastposition ausgeprägter. Sie betrug $20 \mathrm{~mm}$ (Vorlast links) bzw. $15 \mathrm{~mm}$ (Vorlast rechts). Während eine laterale Vorlast vor der Resektion zu einer Vergrößerung des sagittalen Durchmessers geführt hatte, zeigte sich nun also eine Verkleinerung, besonders bei Vorlasten auf der Seite des resezierten Gelenks. 


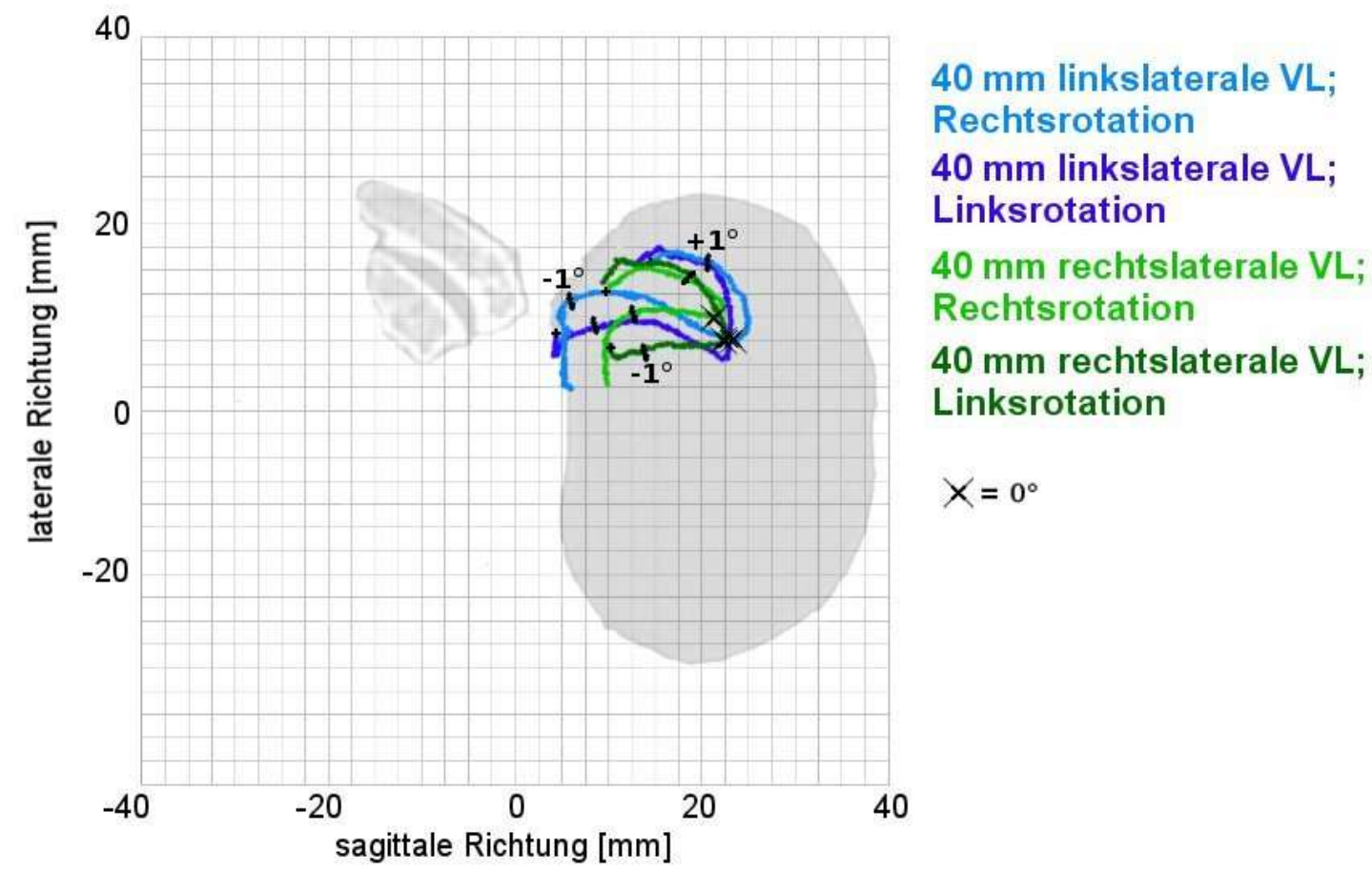

Abbildung 33: Rastpolkurven des Präparats C nach Resektion des rechten Zwischenwirbelgelenks bei lateralen Vorlasten (200 N). Die Kurven befanden sich auch bei rechts-lateraler Vorlast in der linken Bandscheibenhälfte. Axialrotation; die Lage des Wirbels ist eingeblendet; + markiert den Bewegungsbeginn; die Markierungen mit Zahlen beschreiben das Rotationsausmaß.

\section{Bandscheiben-Bänder-Präparat}

Im Vergleich zur zentralen Vorlast kam es durch Lateralverschiebung der Vorlast nicht zu wesentlichen Veränderungen.

\subsubsection{Veränderung der Größe der Vorlast}

Wurden Messungen ohne Vorlast durchgeführt und mit denen von $200 \mathrm{~N}$ verglichen, fand sich eine Verschiebung der Rastpolkurven nach dorsal. Nach Erhöhung der Vorlast auf $400 \mathrm{~N}$ lagen die Kurven wenige Millimeter nach ventral verlagert. Die Form der Kurven betreffend waren die Veränderungen uneinheitlich. Da sich nach jedem Resektionsschritt die gleichen Effekte zeigten, werden sie nicht separat ausgeführt.

\subsubsection{Ausrichtung der Schraubachsen}

Im Gegensatz zum Versuch mit dem Bandscheibenmodell verlief die Rotationsachse im intakten Segment nicht exakt parallel zur z-Achse. Zusätzlich verkippten die Schraubachsen während der Drehbewegung. Abhängig von der Vorlastposition ergaben sich die im Folgenden dargestellten Schraubachslagen. 


\subsubsection{Sagittale Vorlastpositionen}

\section{Intaktes Segment}

Platzierte man die Vorlast zentral, erkannte man anhand der Richtungskomponenten bei einer Rotation von $\alpha \approx 0^{\circ}$ (Neutralstellung) eine Neigung der Schraubachse um 2,7 nach ventral (Tabelle 5), eine laterale Verkippung fand nicht statt (Tabelle 6). Im Laufe der Rechtsrotation kippte die Achse bis auf ein Maximum von $3,4^{\circ}$ nach ventral. Gleichzeitig kam es zu einer Rechtsneigung der Schraubachse bis auf $14,6^{\circ}$. Im maximal linksrotierten Punkt bestand eine Verkippung von $6,4^{\circ}$ nach ventral und $14,3^{\circ}$ nach links. Es kam also bei Persistenz einer Ventralneigung zur Seitkippung in Rotationsrichtung.

Bei $60 \mathrm{~mm}$ ventral positionierter Vorlast war die Schraubachse während der gesamten Rotation dorsal geneigt (Tabelle 5). Die Mittelwerte lagen zwischen $10,2^{\circ}$ und $14^{\circ}$. Bei $\alpha=0^{\circ}$ fand sich keine laterale Neigung. Im Verlauf der Rotationsbewegung verkippte die Achse entgegen der Drehrichtung, d.h. bei Linksrotation nach rechts und bei Rechtsrotation nach links (Tabelle 6). Nach etwa zwei Dritteln des Bewegungsausmaßes erreichte die Verkippung inr Maximum und verringerte sich bei noch weiterer Rotation wieder. Abbildung 34 zeigt zum besseren Verständnis dreidimensional die Lage der Schraubachsen während der beschriebenen Rotation.

Verschob man die Vorlast in dorsale Positionen $(60 \mathrm{~mm})$ war die Schraubachse bei $\alpha=0^{\circ}$ stärker als bei zentraler Vorlast nach ventral verkippt $\left(9,6^{\circ}\right.$ vs. $\left.2,7^{\circ}\right)$. Bei Rotationen von $\alpha \neq 0^{\circ}$ blieb diese ventrale Neigung konstant (Tabelle 5), zusätzlich kam es zu einer lateralen Verkippung in Rotationsrichtung (Tabelle 6). Das Maximum dieses Abkippens fiel mit dem Rotationsmaximum zusammen (Abbildung 34).
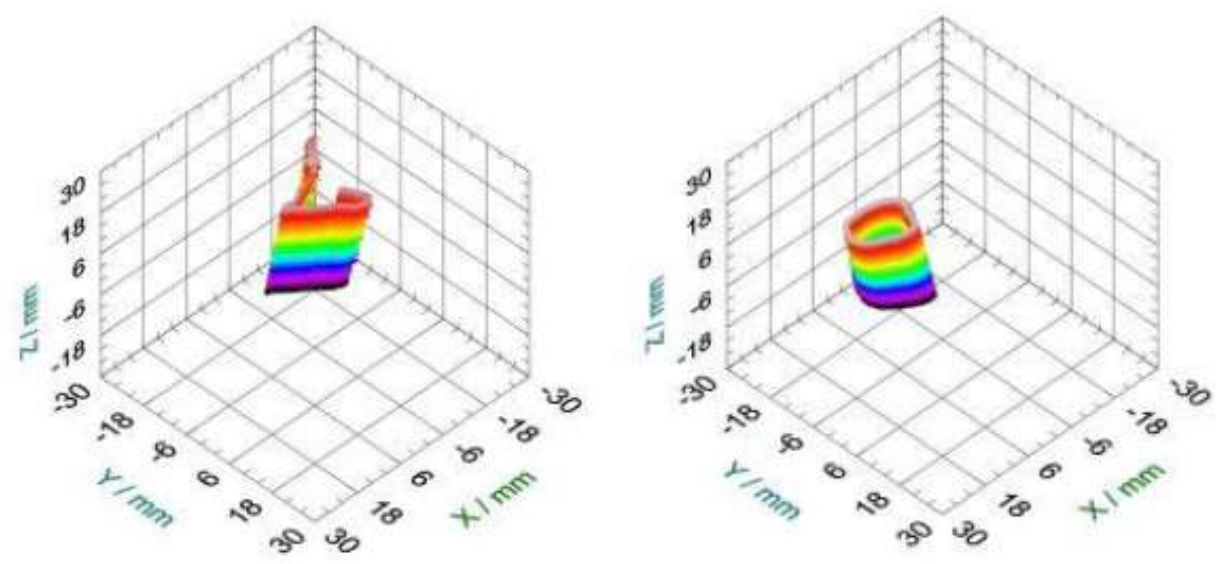

Abbildung 34: 3-D-Aufzeichnung der Wanderung der IHA bei ventraler (links) und dorsaler (rechts) Vorlastposition; intaktes Präparat C; Axialrotation

\section{Segment nach Resektion des rechten Zwischenwirbelgelenks}

Bei zentraler Vorlastposition waren die Schraubachsen in Neutralstellung $\left(\alpha=0^{\circ}\right)$ nach ventral verkippt (Tabelle 5). Bei einer Rotation nach rechts vergrößerte sich der 
Kippungswinkel nach ventral, gleichzeitig neigte sich die Schraubachse nach rechts. War $\alpha>0^{\circ}$ verringerte sich die ventrale Komponente, drei der Präparate zeigten sogar eine nach dorsal geneigte Schraubachse. Die laterale Abweichung erfolgte nun nach links (Tabelle 6). Im Vergleich zum intakten Segment war die ventrale Neigung in rechtsrotierter Stellung $\left(9,3^{\circ}\right.$ vs. $\left.3,4^{\circ}\right)$ und geringer auch in Neutralstellung $\left(4,6^{\circ}\right.$ vs. $\left.2,7^{\circ}\right)$ vergrößert, in Linksdrehung deutlich verringert $\left(3,2^{\circ}\right.$ dorsal vs. $6,4^{\circ}$ ventral). Die laterale Verkippung war stets ausgeprägter als vor der Resektion.

Bei $60 \mathrm{~mm}$ ventral platzierter Vorlast waren die Schraubachsen von vier Präparaten permanent nach dorsal geneigt. Das Ausmaß dieser Neigung war um ca. $1-2^{\circ}$ geringer als vor der Resektion (Tabelle 5). Im Laufe der Bewegung neigten sich die Achsen wie auch im intakten Segment entgegen der Drehrichtung, d.h. im linksrotierten Zustand nach rechts, in rechtsrotierter Stellung nach links. Das Maximum des lateralen Abkippens wurde im letzten Drittel der Rotation erreicht (Tabelle 6).

War die Vorlast $60 \mathrm{~mm}$ dorsal positioniert, neigten sich die Schraubachsen nach ventral (Tabelle 5). Der Neigungswinkel war größer als bei zentraler Vorlast $\left(10^{\circ}\right.$ gegenüber $\left.4,6^{\circ}\right)$. Bei drei der vier Präparate war die ventrale Verkippung in linksrotierter Stellung größer als in Neutral- und rechtsrotierter Position (Tabelle 6). Das Rotationsmaximum entsprach weitgehend dem Zeitpunkt der größten lateralen Verkippung in Drehrichtung. Während die Ventralneigung im intakten Segment im Bewegungsverlauf unverändert geblieben war, wurde nun im Mittel eine kontinuierliche Zunahme im Laufe der Linksrotation aufgezeichnet.

\section{Bandscheiben-Bänder-Präparat}

Die Schraubachsen waren in zentraler Vorlastposition wie auch im intakten Segment während der gesamten Drehbewegung nach ventral verkippt. Die durchschnittliche Ventralneigung war mit $4,9^{\circ}$ bei Drehwinkeln von $\alpha=0^{\circ}$ am größten, zum Zeitpunkt der maximalen Rechts- bzw. Linksrotation auf 2,9 $9^{\circ}$ bzw. 3,1 $1^{\circ}$ verringert (Tabelle 5). Während das Ausmaß der Neigung bei $\alpha=0^{\circ}$ größer war als im unresezierten Zustand, war es in den Rotationsmaxima um $0,5^{\circ}$ bis $3,3^{\circ}$ kleiner. Die stärkste laterale Abweichung - jeweils in Rotationsrichtung - fand sich auch nach der Resektion beider Zwischenwirbelgelenke etwa im letzten Drittel der Rotation. Die Schraubachsen waren bei Rechtsdrehung um $5^{\circ}$ nach rechts, bei Linksdrehung um $12,2^{\circ}$ nach links geneigt (Tabelle 6). Auch in Neutralstellung wurden Neigungstendenzen nach lateral festgestellt.

Durch eine $40 \mathrm{~mm}$ ventral positionierte Vorlast kam es zu einer Verlagerung der Schraubachsen. Während sie in Neutralstellung um $5,2^{\circ}$ nach dorsal geneigt waren, kippten sie bei positiven und negativen Drehwinkeln auf $1,2^{\circ}$ bzw. $2^{\circ}$ nach ventral, was im intakten Segment nicht zu beobachten gewesen war (Tabelle 5). Es kam weiterhin zu einer lateralen Neigung, nun entgegen der Rotationsrichtung, d.h. in Rechtsrotation um $11,7^{\circ}$ nach links, in Linksrotation um 5,5 nach rechts (Tabelle 6).

Die Ausrichtung der Achsen bei $20 \mathrm{~mm}$ dorsal angebrachten Vorlastpositionen entsprach weitgehend der bei zentraler Last: Sie waren im gesamten Bewegungs- 
ablauf nach ventral geneigt (Tabelle 5) und im Rotationsmaximum in Rotationsrichtung verkippt (Tabelle 6). Im Unterschied zur zentralen Vorlast war die ventrale Abweichung mit $6,5^{\circ}$ bei $\alpha=0^{\circ}$ und $4,2^{\circ}$ bzw. $4,1^{\circ}$ in den Rotationsmaxima stärker ausgeprägt. Im Vergleich zum unresezierten Zustand war die permanente Ventral- sowie Lateralneigung bei großen Drehwinkeln geringer. Es fiel jedoch ein deutliches laterales Abkippen $\left(6,6^{\circ}\right.$ nach rechts) in Neutralstellung auf.

Tabelle 5: Mittelwerte der ventro-dorsalen Abweichung der momentanen Schraubachse von der Vertikalen in Grad bei unterschiedlichen Segmentzuständen, Vorlastpositionen (VL) und Rotationsstadien; Standardabweichung in Klammern. Unabhängig vom Resektionsstatus führten dorsale Vorlasten zu dorsal geneigten IHA, ventrale zu ventral geneigten. Das Ausmaß der Neigung ist stets variabel. $\alpha=$ Rotationswinkel; + bzw. - = Abweichung nach ventral bzw. dorsal; Axialrotation

\begin{tabular}{|c|c|cc|cc|cc|}
\hline Segmentzustand & VL-Position & \multicolumn{3}{|c|}{$\boldsymbol{\alpha}=\mathbf{0}^{\circ}$} & max. rechtsrotiert & max. linksrotiert \\
\hline \multirow{4}{*}{\begin{tabular}{c} 
intaktes Segment \\
\cline { 2 - 9 }
\end{tabular}} & zentral & 2,7 & $( \pm 5,0)$ & 3,4 & $( \pm 4,3)$ & 6,4 & $( \pm 3,9)$ \\
\cline { 2 - 9 } & ventral & $-10,2$ & $( \pm 6,0)$ & $-14,0$ & $( \pm 8,6)$ & $-11,5$ & $( \pm 4,0)$ \\
\cline { 2 - 9 } & dorsal & 9,6 & $( \pm 2,6)$ & 9,4 & $( \pm 2,5)$ & 9,4 & $( \pm 3,1)$ \\
\hline \multirow{4}{*}{$\begin{array}{c}\text { nach Resektion des } \\
\text { rechten Gelenks }\end{array}$} & zentral & 4,6 & $( \pm 2,1)$ & 9,3 & $( \pm 7,5)$ & $-3,2$ & $( \pm 7,9)$ \\
\cline { 2 - 9 } & ventral & $-8,5$ & $( \pm 4,7)$ & $-13,4$ & $( \pm 7,8)$ & $-10,3$ & $( \pm 2,4)$ \\
\cline { 2 - 9 } & dorsal & 10,0 & $( \pm 7,3)$ & 7,8 & $( \pm 7,1)$ & 13,8 & $( \pm 11,1)$ \\
\hline \multirow{3}{*}{$\begin{array}{c}\text { Bandscheiben-Bänder- } \\
\text { Präparat }\end{array}$} & zentral & 4,9 & $( \pm 2,9)$ & 2,9 & $( \pm 5,3)$ & 3,1 & $( \pm 3,9)$ \\
\cline { 2 - 8 } & ventral & $-5,2$ & $( \pm 6,2)$ & 2,0 & $( \pm 6,5)$ & 1,2 & $( \pm 5,7)$ \\
\cline { 2 - 8 } & dorsal & 6,5 & $( \pm 2,6)$ & 4,2 & $( \pm 4,7)$ & 4,1 & $( \pm 1,6)$ \\
\hline
\end{tabular}

Tabelle 6: Mittelwerte der lateralen Abweichung der momentanen Schraubachse von der Vertikalen in Grad bei unterschiedlichen Segmentzuständen, Vorlastpositionen (VL) und Rotationsstadien; Standardabweichung in Klammern. Die Richtung der lateralen Abweichung in den Rotationsmaxima war unabhängig vom Resektionsstatus - bei ventraler Vorlastposition entgegengesetzt zu jener bei zentraler und dorsaler Vorlast. $\alpha=$ Rotationswinkel; + bzw. - = Abweichung nach links bzw. rechts; Axialrotation

\begin{tabular}{|c|c|cc|cc|cc|}
\hline Segmentzustand & VL-Position & \multicolumn{3}{|c|}{$\boldsymbol{\alpha}=\mathbf{0}^{\circ}$} & max. rechtsrotiert & max. linksrotiert \\
\hline \multirow{4}{*}{ intaktes Segment } & zentral & $-0,1$ & $( \pm 0,7)$ & $-14,6$ & $( \pm 4,4)$ & 14,3 & $( \pm 2,6)$ \\
\cline { 2 - 9 } & ventral & 0,0 & $( \pm 0,0)$ & 9,0 & $( \pm 6,0)$ & $-8,4$ & $( \pm 3,4)$ \\
\cline { 2 - 9 } & dorsal & 0,0 & $( \pm 0,0)$ & $-16,0$ & $( \pm 3,1)$ & 12,5 & $( \pm 5,8)$ \\
\hline \multirow{4}{*}{$\begin{array}{c}\text { nach Resektion des } \\
\text { rechten Gelenks }\end{array}$} & zentral & 0,0 & $( \pm 0,0)$ & $-15,9$ & $( \pm 3,7)$ & 19,9 & $( \pm 7,5)$ \\
\cline { 2 - 9 } & ventral & 0,0 & $( \pm 0,0)$ & 8,4 & $( \pm 5,6)$ & $-10,7$ & $( \pm 5,9)$ \\
\cline { 2 - 8 } & dorsal & $-0,2$ & $( \pm 0,6)$ & $-7,2$ & $( \pm 12,6)$ & 12,5 & $( \pm 7,6)$ \\
\hline \multirow{3}{*}{$\begin{array}{c}\text { Bandscheiben-Bänder- } \\
\text { Präparat }\end{array}$} & zentral & 1,2 & $( \pm 3,1)$ & $-5,0$ & $( \pm 11,7)$ & 12,2 & $( \pm 9,1)$ \\
\cline { 2 - 8 } & ventral & $-0,4$ & $( \pm 0,9)$ & 11,7 & $( \pm 14,4)$ & $-5,5$ & $( \pm 15,9)$ \\
\cline { 2 - 8 } & dorsal & $-6,6$ & $( \pm 7,7)$ & $-8,3$ & $( \pm 6,9)$ & 7,7 & $( \pm 19,6)$ \\
\hline
\end{tabular}


Die Abbildung 35 zeigt zum besseren Vergleich die durchschnittliche Abweichung der Schraubachse in der sagittalen Ebene, also nach ventral und dorsal, der drei Resektionsschritte. Es zeigte sich, dass sich die Abweichungswinkel nach der ersten und zweiten Resektion besonders bei dorsalen Vorlastpositionen glichen, während die des intakten Segments durchgängig etwas geringer waren. Im Vorlastbereich um den Nullpunkt herum blieb die Achslage innerhalb einer Resektionsstufe nahezu konstant. Beim intakten Segment kam es lediglich in der Vorlastposition null zu dezent größeren Winkeln, beim Bandscheiben-Bänder-Präparat hingegen zu kleineren Abweichungen. Auffällig war weiterhin, dass die Variabilität innerhalb der Segmente (Standardabweichung) im intakten Segment umso stetiger abnahm, je dorsaler die Vorlast gelegen war. Nach der Resektion des rechten Zwischenwirbelgelenks fand sich die minimale Variabilität bei $20 \mathrm{~mm}$ ventralen Vorlasten, beim Bandscheiben-Bänder-Präparat lagen nach dorsal eher zunehmende Standardabweichungen vor.

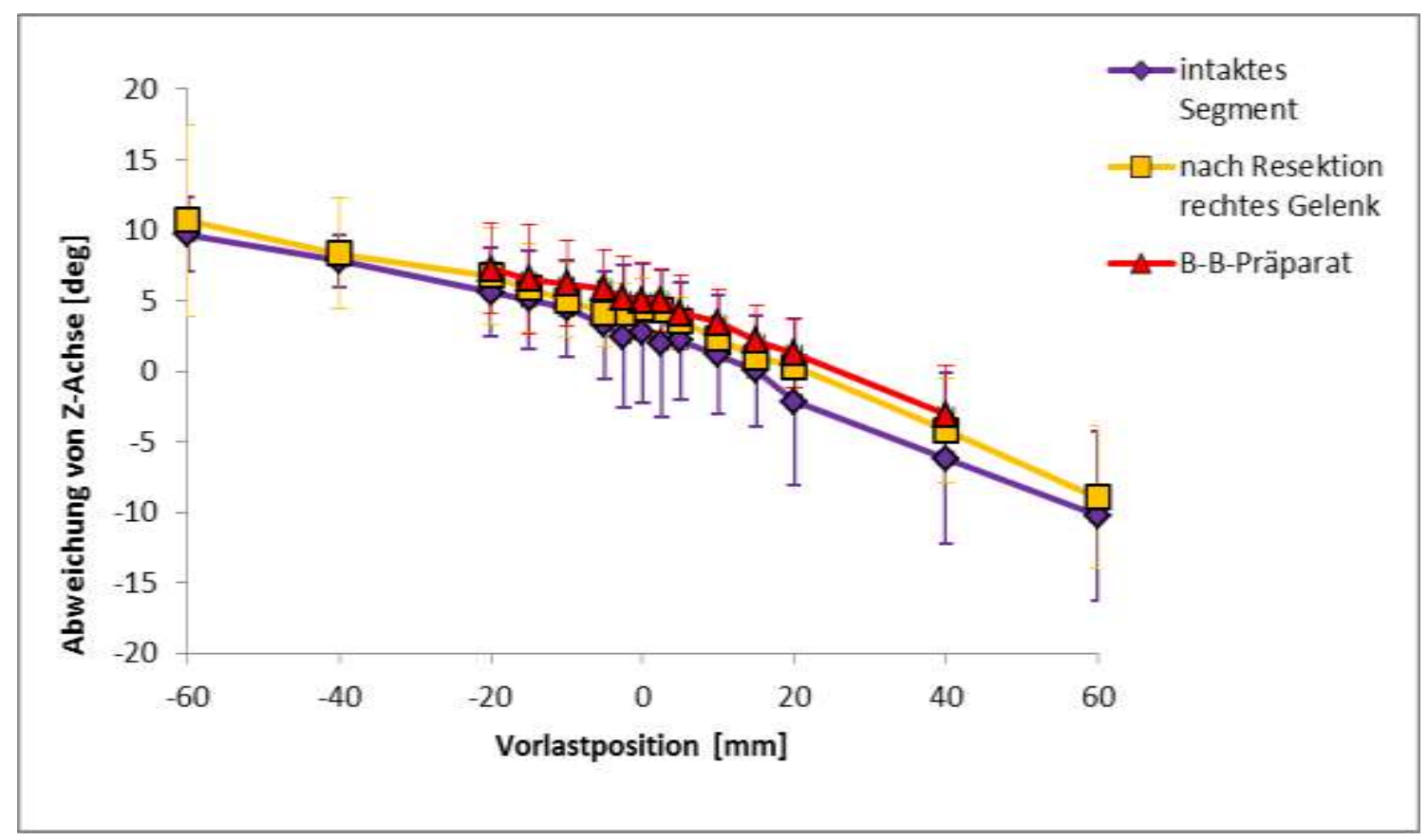

Abbildung 35: Mittlere ventro-dorsale Abweichung der IHA von der z-Achse in Grad bei allen gemessenen sagittalen Vorlastpositionen; alle Resektionszustände. Unabhängig vom Resektionsstatus führten dorsale Vorlasten zu dorsal geneigten IHA, ventrale zu ventral geneigten. Axialrotation

\subsubsection{Laterale Verschiebung der Vorlast}

\section{$\underline{\text { Intaktes Segment }}$}

Es kam ähnlich wie bei zentraler Vorlast im Rotationsverlauf zu einem lateralen Verkippen der Schraubachse in die Drehrichtung. Allerdings durchlief jetzt auch die sagittale Komponente einen Vorzeichenwechsel. Bei linksseitiger Vorlast und in linksrotierter Stellung lag die Schraubachse ventral, in Rechtsrotation dorsal des Mittelpunkts. Umgekehrt verhielt es sich bei rechts platzierter Vorlast. Dann erfolgte die ventrale Verkippung bei Rechtsrotation, die dorsale bei Linksrotation. Die Ventralneigung erschien in beiden Vorlastrichtungen ausgeprägter als die dorsale. Bei einigen Präpa- 
raten erfolgte der sagittale Vorzeichenwechsel nicht bei $\alpha=0^{\circ}$, sondern bei kleinen Drehwinkeln in Richtung der Vorlastposition, d.h. bei linker Vorlast, wenn $\alpha<0^{\circ}$ und vice versa.

\section{Segment nach Resektion des rechten Zwischenwirbelgelenks}

War die Vorlast linksseitig angebracht, kippten die Schraubachsen in rechtsrotierter Stellung nach dorsal und rechts, in linksrotierter Stellung nach ventral und links. Bei rechten Vorlastpositionen neigten sie sich in maximaler Rechtsrotation nach ventral und rechts, in maximaler Linksrotation nach dorsal und links. Dies ist vergleichbar mit den Ergebnissen vor der Resektion. Geht man von einer Linksdrehung aus, erfolgte der Wechsel zwischen ventral und dorsal bei linksseitig angebrachter Vorlast nun jedoch eher als bei rechtsseitiger Vorlast. Die Verkippung in der Sagittalen war bei Rotationen zur jeweiligen Seite ausgeprägter, an der die Vorlast angebracht war. Weiterhin wurde bei Betrachtung der maximalen Rechtsrotation deutlich, dass bei rechts positionierter Vorlast die Verkippung nach rechts und besonders nach ventral stärker war als die rechts-dorsale Neigung bei linksseitiger Vorlast. Dahingegen verringerten sich die entsprechenden Veränderungen bei maximaler Linksdrehung. Bei den Messungen mit dem intakten Segment konnten keine seitenspezifischen Unterschiede festgestellt werden.

\section{Bandscheiben-Bänder-Präparat}

Wurde die Vorlast linksseitig positioniert, kam es im Verlauf der Drehbewegung zu einem Vorzeichenwechsel der sagittalen Komponente. In maximaler Rechtsrotation war die Schraubachse nach dorsal und rechts geneigt. Während der Bewegung zur Neutralstellung kippte sie nach ventral. Der ventrale Neigungswinkel nahm im Linksrotationsverlauf stetig zu. Die laterale Abweichung erfolgte von rechts nach links.

Bei rechten Vorlastpositionen verhielt sich die laterale Komponente der Schraubachse analog, die sagittale wie gespiegelt. In maximaler Rechtsrotation war sie nach ventral und rechts geneigt, in Linksrotation nach dorsal und links. Der Vorzeichenwechsel der sagittalen Komponente verlagerte sich auf den Bereich zwischen Neutral- und linksrotierter Stellung. Analog zu den Ergebnissen bei linker Vorlastposition war das ventrale Verkippen im rechtsgedrehten Zustand am größten.

Somit war die Ausrichtung der Schraubachsen vergleichbar mit der des intakten Segments. Allerdings waren sowohl die ventralen als auch die lateralen Neigungswinkel nun kleiner. Der sagittale Vorzeichenwechsel fand öfter und bei größeren Drehwinkeln statt als vor den Resektionen.

\subsubsection{Veränderung der Größe der Vorlast}

Eine Verringerung der Vorlast führte beim intakten Segment zu einem Abkippen der momentanen Schraubachse nach dorsal, eine Erhöhung nach ventral. Unter beiden Bedingungen fand in Maximalrotation eine Lateralneigung in Rotationsrichtung statt. Die Veränderungen, bedingt durch die Resektion des rechten Gelenks, waren nicht 
einheitlich. Die Schraubachslage der Bandscheiben-Bänder-Präparate ähnelte der des intakten Segments, jedoch mit geringeren Abweichungen von der z-Achse.

\subsubsection{Drehwinkel-Drehmoment-Kennlinie und Bewegungsausmaß}

\subsubsection{Sagittale Verschiebung der Vorlast}

Charakteristisch für die Drehwinkel-Drehmoment-Kennlinie war die sigmoide Form und das Vorhandensein einer Hysterese $\Delta \alpha$. Dies galt für alle Präparate in allen gemessenen Vorlastpositionen (Abbildung 36). Die Breite der Hysterese zeigte eine Verringerung von $1,07^{\circ}$ auf $0,63^{\circ}$ bei dorsalen Vorlastpositionen, während sie bei Ventralverschiebung nahezu unverändert blieb.

Nach dem ersten Resektionsschritt behielt die Drehwinkel-Drehmoment-Kennlinie eine sigmoide Form. Die Breite der Hysterese war generell größer als bei intaktem Segment (bei zentraler Vorlast $1,46^{\circ}$ gegenüber $1,07^{\circ}$ vor der der Resektion). Bei Ventralverschiebung waren die Veränderungen uneinheitlich. Im Mittel kam es jedoch sowohl bei Ventral- als auch bei Dorsalverschiebung zu einer Verringerung der Hysterese auf $1,26^{\circ}$ (ventral) und $0,87^{\circ}$ (dorsal).

Bei Messung der Bandscheiben-Bänder-Präparate war die sigmoide Form der Kennlinie bei den verschiedenen Präparaten unterschiedlich stark ausgeprägt. Sie wurde besonders bei ventraler Vorlastposition stärker, nahm bei Verschiebung der Last nach dorsal jedoch ab (Abbildung 36). Die Breite der Hysterese hingegen verkleinerte sich bei ventralen Vorlasten deutlich, während sie bei Dorsalverschiebung weitgehend konstant blieb. Aus dem Vergleich zum intakten Segment ergab sich eine Vergrößerung der Hysteresenbreite auf jeweils etwa $1,5^{\circ}$ bei zentraler bzw. dorsaler Vorlast, sowie eine Verkleinerung bei ventraler Vorlast auf $0,87^{\circ}$.
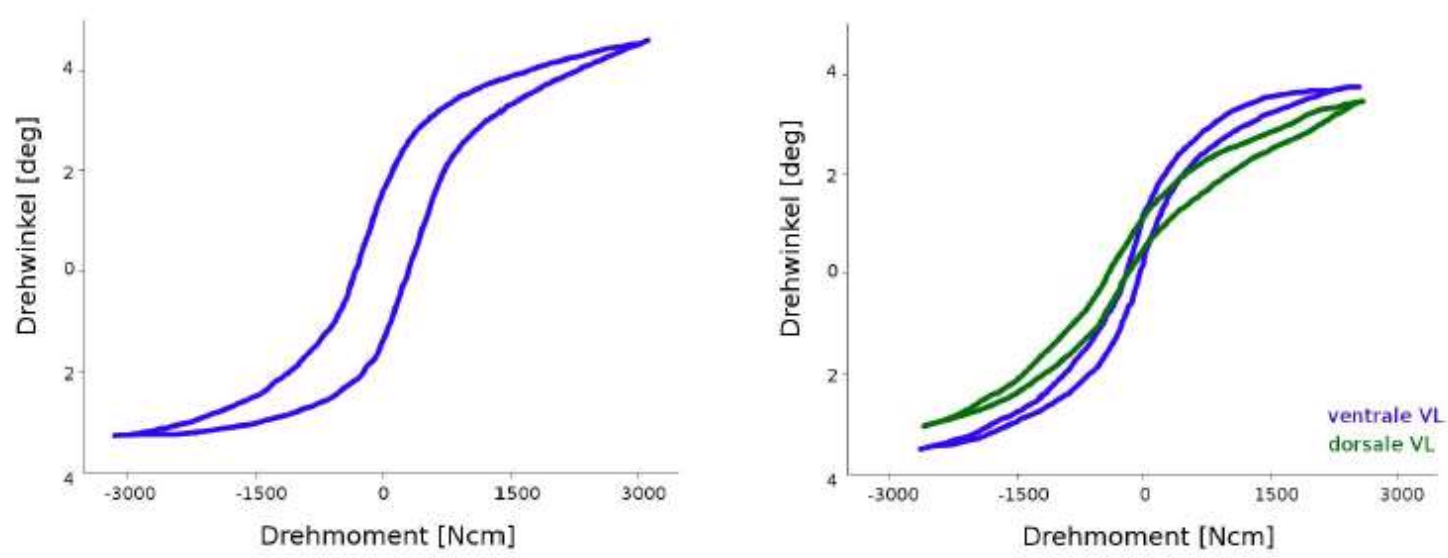

Abbildung 36: Sigmoide Drehwinkel-Drehmoment-Kennlinie des intakten Präparats D bei zentraler Vorlast (links) sowie des Bandscheiben-Bänder-Präparats bei je $60 \mathrm{~mm}$ ventraler und dorsaler Vorlast (rechts); Axialrotation. Die Sigmoidität wurde durch ventrale Vorlastpositionen verstärkt, durch dorsale vermindert. 


\section{Bewegungsausmaß (ROM): Intaktes Segment}

Bei Betrachtung des Bewegungsausmaßes konnte eine deutliche Abhängigkeit von der Vorlastposition festgestellt werden. So vergrößerte sich der ROM bei Verschiebung von zentral $\left(5,5^{\circ}\right)$ nach ventral $\left(6,75^{\circ}\right)$ stetig (Tabelle 7$)$. Wurde die Vorlast weiter in dorsale Richtung verschoben, nahm das Bewegungsausmaß zunächst zu $\left(6,2^{\circ}\right)$, um dann ab einer dorsalen Position von $5 \mathrm{~mm}$ erneut auf $5,4^{\circ}$ abzunehmen (Abbildung 37). Prozentual ergaben sich mit Referenz auf zentrale Vorlast Werte zwischen $124 \%$ und $99 \%$.

\section{Segment nach Resektion des rechten Zwischenwirbelgelenks}

Der ROM war im Vergleich zu den Ergebnissen bei intaktem Segment gestiegen (auf $133 \%$ bei zentraler Vorlast). Besonders die Drehung nach links führte zu größeren Rotationswinkeln. Das Bewegungsausmaß lag bei zentraler Vorlastposition bei $7,3^{\circ}$ und stieg bis zum Maximum bei ventralen Vorlastpositionen $\left(8,4^{\circ}\right)$ stetig an. Bei einer 2,5 $\mathrm{mm}$ dorsal platzierten Vorlast blieb es annähern konstant, um dann bei weiterer Vorlastverschiebung nach dorsal bis $6,1^{\circ} \mathrm{zu}$ sinken. Die Vergrößerung des range of motion bei ventralen Vorlastpositionen betrug nach der Resektion lediglich $116 \%$ des Wertes bei zentraler Vorlast und war damit weniger ausgeprägt als beim intakten Segment. Eine weitere Dorsalverschiebung führte dagegen zu einer Verringerung auf $82 \%$ und somit zu einem stärkeren Abfall als vor der Resektion (Tabelle 7, Abbildung 37).

\section{Bandscheiben-Bänder-Präparat}

Nach der Resektion beider Bogengelenke war der range of motion - ähnlich dem intakten Zustand - bei zentraler Vorlast am geringsten (Abbildung 37). Er nahm bei $10 \mathrm{~mm}$ dorsal und $20 \mathrm{~mm}$ ventral platzierten Vorlasten um ca. $2^{\circ}$ auf $119 \%$ bzw. $123 \%$ zu (Tabelle 7). Es gab jedoch größere interindividuelle Varianz.

Tabelle 7: Mittlerer ROM in Grad absolut und in Prozent des ROM bei zentraler Vorlast bei verschiedenen sagittalen Vorlastpositionen; alle Segmentzustände; Standardabweichung in Klammern; Axialrotation. Der ROM nahm nach jedem Resektionsschritt und durch Verschiebung der Vorlast - besonders nach ventral zu. Maximal dorsale Vorlasten führten zum Absinken des ROM.

\begin{tabular}{|c|c|c|c|c|c|c|}
\hline $\begin{array}{c}\text { Segmentzustand/ } \\
\text { Vorlastposition (mm) }\end{array}$ & $\begin{array}{c}\mathbf{6 0} \\
\text { dorsal }\end{array}$ & $\begin{array}{c}\mathbf{1 0} \\
\text { dorsal }\end{array}$ & $\begin{array}{c}\mathbf{2 , 5} \\
\text { dorsal }\end{array}$ & zentral & $\begin{array}{c}\mathbf{2 0} \\
\text { ventral }\end{array}$ & $\begin{array}{c}\mathbf{6 0} \\
\text { ventral }\end{array}$ \\
\hline intaktes Segment & $5,4( \pm 1,07)$ & $6,3( \pm 1,39)$ & $6,2( \pm 1,4)$ & $5,5( \pm 1,15)$ & $6,3( \pm 1,17)$ & $6,75( \pm 1,09)$ \\
\hline $\begin{array}{c}\mathbf{9 9} \% \\
\text { nach Resektion des } \\
\text { rechten Gelenks }\end{array}$ & $6,1( \pm 1,34)$ & $\mathbf{1 1 2} \%$ & $\mathbf{1 1 0} \%$ & $\mathbf{1 0 0 \%}$ & $\mathbf{1 1 4} \%$ & $\mathbf{1 2 4} \%$ \\
\hline $\begin{array}{c}\text { Bandscheiben- } \\
\text { Bänder-Präparat }\end{array}$ & $\mathbf{8 2} \%$ & $\mathbf{9 9} \%$ & $\mathbf{1 0 5} \%$ & $\mathbf{1 0 0} \%$ & $\mathbf{1 1 0} \%$ & $\mathbf{1 1 6} \%$ \\
\hline
\end{tabular}




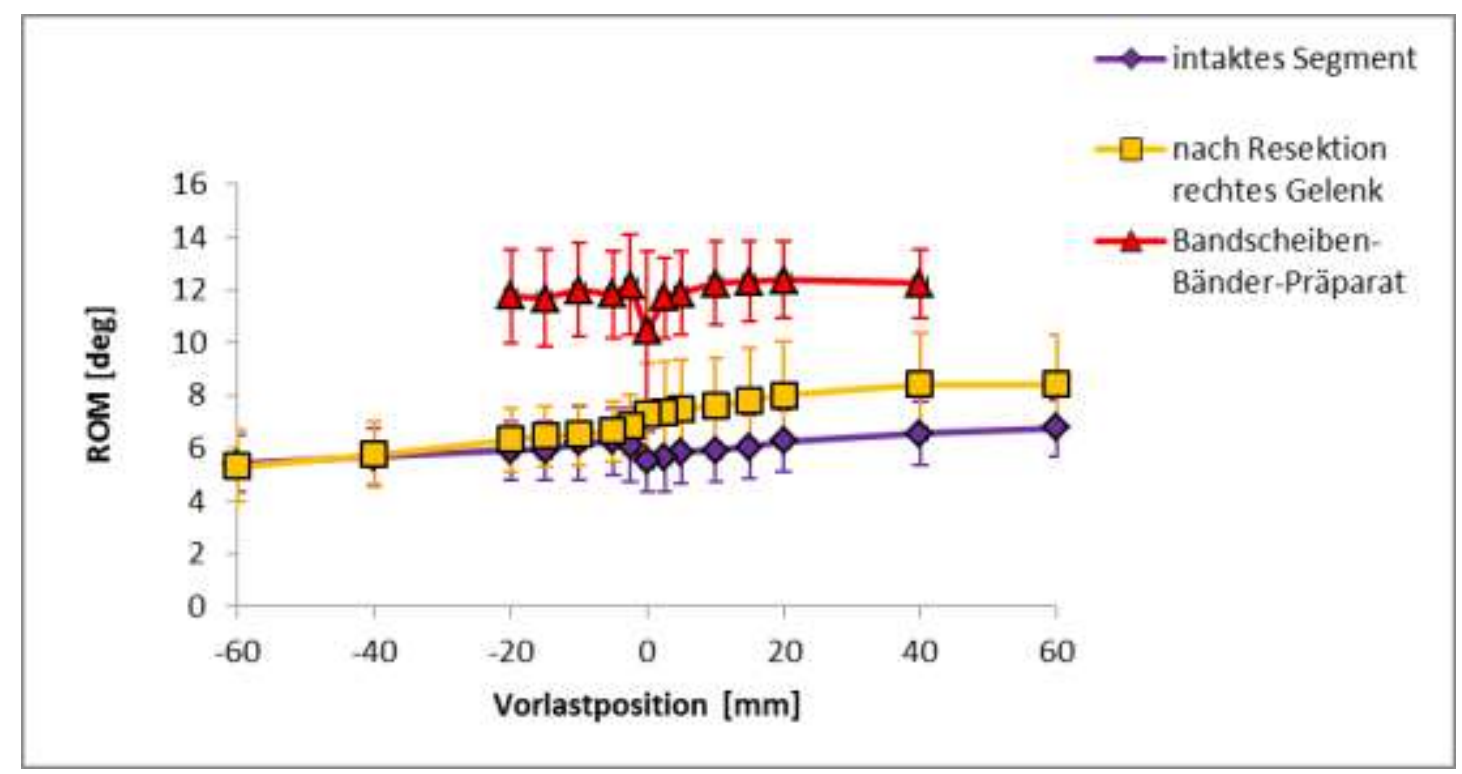

Abbildung 37: Mittlerer ROM in Grad bei allen gemessenen sagittalen Vorlastpositionen (Vorlast $200 \mathrm{~N}$ ). Alle Resektionszustände zeigten ein Absinken des ROM bei gering dorsalen Vorlasten. Beim intakten Segment und Bandscheiben-Bänder-Präparat führte eine weitere Dorsalverschiebung zunächst wieder zu einem Anstieg des ROM. Alle Resektionszustände; Axialrotation

\subsubsection{Laterale Verschiebung der Vorlast}

Platzierte man die Vorlast bei intaktem Segment lateral, war die Breite der Hysterese meist ähnlich der bei zentraler Position. Bei linksseitig angebrachter Vorlast zeigte sich eine Verringerung von $1,07^{\circ}$ auf $0,85^{\circ}$ während rechtsseitig keine Veränderung stattfand. Dieser Verringerungseffekt trat nach jeder Resektion beidseits und ausgeprägter auf.

\section{Bewegungsausmaß (ROM): Intaktes Segment}

Der ROM war bei zentraler Vorlast am geringsten. Verschob sich die Vorlast um $5 \mathrm{~mm}$ nach lateral, konnte eine Vergrößerung des Bewegungsausmaßes um bis zu $1.5^{\circ}$ festgestellt werden. Bei weiterer Lateralisation waren nur noch geringe ROMZunahmen zu verzeichnen (Abbildung 38). Das mittlere Bewegungsausmaß bei zentraler Vorlast betrug $5,5^{\circ}$ bei linker bzw. rechter Vorlast $6,3^{\circ}$ bzw. $6,35^{\circ}$. Wurde der Wert bei zentraler Vorlast wiederum mit $100 \%$ definiert, ergab sich jeweils eine Erhöhung auf ca. $115 \%$.

\section{Segment nach Resektion des rechten Zwischenwirbelgelenks}

Das Bewegungsausmaß blieb ab einer lateralen Vorlastverschiebung von 5-10 mm in etwa konstant. Es zeigte sich kein wesentlicher Unterschied zwischen links- und rechts-lateral platzierter Vorlast. Damit sind die Ergebnisse in ihrer Tendenz vergleichbar mit denen im intakten Segment. Der kleinste ROM fand sich weiterhin bei zentraler Vorlast. (Abbildung 38). Mit $7^{\circ}$ bei linken, $6,6^{\circ}$ bei zentralen und $6.8^{\circ}$ bei rechten Vorlasten war der range of motion höher als im unresezierten Zustand. Prozentual ergab sich jedoch eine Erhöhung auf lediglich $106 \%$ bzw. $103 \%$. Somit war das Bewegungsausmaß zwar größer, der Unterschied durch Variation der Vorlastposition aber geringer als vor der Resektion. 


\section{Bandscheiben-Bänder-Präparat}

Der range of motion war, wie auch im intakten Segment, bei zentraler Vorlast geringer als bei lateralen Lasten (Abbildung 38). Im Durchschnitt zeigte sich bei zentraler Vorlast ein Bewegungsausmaß von $10,4^{\circ}$, bei $20 \mathrm{~mm}$ links- bzw. rechtsseitiger Position Werte von $13^{\circ}$ bzw. $12,5^{\circ}$. Im Vergleich zum Zustand vor den Resektionen lag demnach auch bei lateralen Vorlasten etwa eine Verdopplung des Bewegungsausmaßes vor. Zwischen linker und rechter Vorlast konnten Unterschiede von bis zu $1,5^{\circ}$ festgestellt werden.

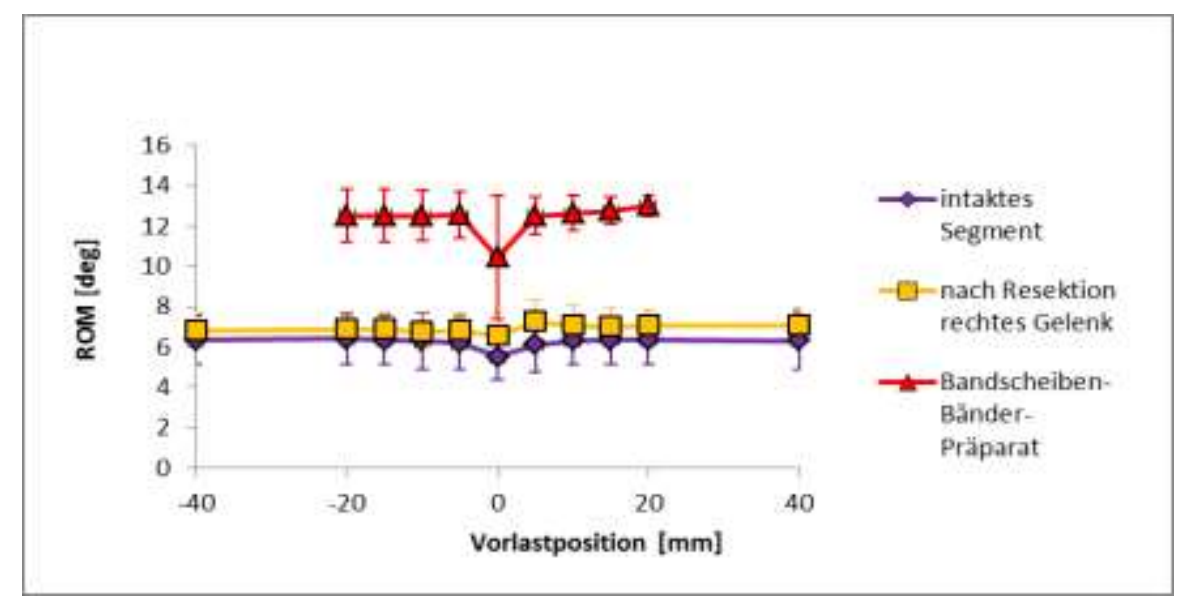

Abbildung 38: Mittlerer ROM in Grad bei allen gemessenen lateralen Vorlastpositionen (Vorlast $200 \mathrm{~N}$ ). Eine Lateralverschiebung der Vorlast führte - besonders beim Bandscheiben-Bänder-Präparat - zu einem Anstieg des ROM; alle Resektionszustände; Axialrotation

\subsubsection{Veränderung der Größe der Vorlast}

Die Messungen am intakten Segment ergaben keine Ergebnisse, die auf regelhafte Veränderungen der Hysteresenbreite schließen lassen. Dahingegen führte nach Resektion des rechten Gelenks eine Verringerung der Vorlast zu breiteren Hysteresen. Eine Erhöhung der Vorlast bedingte dementsprechend schmale Hysteresen. Nach Resektion des zweiten Gelenks lag wiederum Inhomogenität in den Effekten vor.

Wie auch bei Betrachtung der Breite der Hysterese waren Veränderungen des ROM der intakten Segmente und Bandscheiben-Bänder-Präparate durch Variation der Vorlastgröße wenig einheitlich. Nach der ersten Resektion stieg das Bewegungsausmaß durch Elimination der Vorlast auf $104 \%$, sank bei Erhöhung auf $400 \mathrm{~N}$ auf $85 \%$ des Ausgangswertes ab.

\subsubsection{Steifigkeitsverhalten}

Während eines Halbzyklus veränderte sich die Steifigkeit unabhängig vom Resektionszustand der Präparate parabelförmig. Die höchste Steifigkeit fand sich stets an den Umkehrpunkten der Drehrichtung, d.h. in maximal rotierter Stellung des oberen Wirbels. Die minimale Steifigkeit wurde ungefähr zum Zeitpunkt der geringsten Rotation $\left(\alpha \approx 0^{\circ}\right)$ erreicht. Sie verhielt sich umgekehrt proportional zum ROM, d.h. eine 
hohe Steifigkeit ging mit einem kleinen ROM einher und umgekehrt. Nach jedem Resektionsschritt verkleinerte sich der Abstand zwischen minimaler Steifigkeit bei kleinen Drehwinkeln und maximaler Steifigkeit im Bereich großer Rotationswinkel (Abbildung 39). Nach der beidseitigen Gelenkresektion war er halbiert. Gleichzeitig verbreiterte sich die Basis der Parabel.

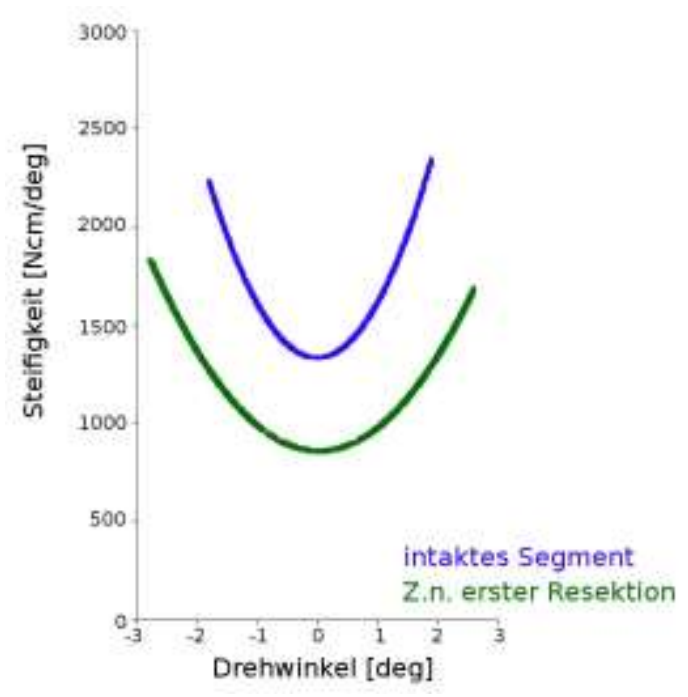

Abbildung 39: Steifigkeitskurven bei zentraler Vorlast (200 N) des Präparats C im intakten Segment und nach Resektion des rechten Zwischenwirbelgelenks. Nach der Resektion war die Gesamtsteifigkeit vermindert und der Kurvenverlauf breiter. Axialrotation

\subsubsection{Sagittale Verschiebung der Vorlast}

\section{Intaktes Segment}

Die minimale Steifigkeit nahm bei ventraler Vorlast im Vergleich zur zentralen von $664 \mathrm{Ncm} / \mathrm{deg}$ auf $318 \mathrm{Ncm} / \mathrm{deg}$ ab. Wenige Millimeter dorsal des Nullpunktes fiel sie rasant bis auf $454 \mathrm{Ncm} / \mathrm{deg}$ ab und stieg bei weiterer Verschiebung nach dorsal stetig bis $762 \mathrm{Ncm} / \mathrm{deg}$ an (Abbildung 40, Tabelle 8). Die Veränderungen um den Nullpunkt herum waren deutlicher ausgeprägt als bei Betrachtung des range of motion.

Die Abbildung 41 zeigt die relative Steifigkeit der Präparate. Diese ergibt sich durch Division der minimalen Steifigkeit $D$ in der Vorlastposition $x(=D(x))$ durch die Steifigkeit bei zentraler Vorlast $D(x=0)$. Man sieht, dass es ausgehend von zentraler Vorlast $(=1)$ zu einer Verringerung der Steifigkeit auf 0,46 , also $46 \% \mathrm{kam}$, wenn die Vorlast um $60 \mathrm{~mm}$ nach ventral verlagert wurde. Dahingegen verringerte sie sich auf 0,73 bei Verschiebung der Vorlast um lediglich 2,5 $\mathrm{mm}$ in dorsale Richtung. Die Werte bei $60 \mathrm{~mm}$ dorsaler Belastung lagen bei $130 \%$ des Wertes bei zentraler Vorlast.

\section{Segment nach Resektion des rechten Zwischenwirbelgelenks}

Bei dezent dorsaler Vorlastposition kam es, im Gegensatz zum intakten Segment, zu einem geringen Absinken der Steifigkeit (Abbildung 40). Dies wird auch bei Betrachtung der relativen Steifigkeit deutlich (Abbildung 41). Der Unterschied zwischen zentraler und 2,5 mm dorsaler Vorlast betrug nun durchschnittlich $11 \%$. Eine weitere Dorsal- 
verschiebung der Vorlast führte innerhalb der ersten $5 \mathrm{~mm}$ zu einem starken, danach zu einem leichten Anstieg der Steifigkeit. Der Steifigkeitszuwachs durch dorsale Vorlasten lag bei $226 \%$. Durch Verschiebung der Last nach ventral wurden $79 \%$ der Steifigkeit bei zentraler Vorlast erreicht (Tabelle 8). Neben der allgemein geringeren Steifigkeit wird deutlich, dass die Veränderungen durch ventrale und leicht dorsale Vorlastpositionen kleiner waren als vor der Resektion des Gelenks, während eine Dorsalverschiebung um $60 \mathrm{~mm}$ zu einem stärkeren Steifigkeitsanstieg führte.

\section{Bandscheiben-Bänder-Präparat}

Die größte Steifigkeit zeigt sich nun bei zentraler Vorlast (253 Ncm/deg). Sowohl die Verschiebung der Last nach ventral als auch nach dorsal führt zu einem zunächst steileren, dann seichten Absinken der Steifigkeit (Abbildung 40). Unter der Annahme, dass die Steifigkeit $D$ in der Vorlastposition 0 gleich 1 sei, ergaben sich 0,68 bzw. 0,74 bei $5 \mathrm{~mm}$ ventralen bzw. dorsalen Vorlasten (Abbildung 41). Mit der am weitesten dorsal gelegenen Vorlastposition wurden 203 Ncm/deg (1,1 in Relation zu Position 0) erreicht (Tabelle 8).

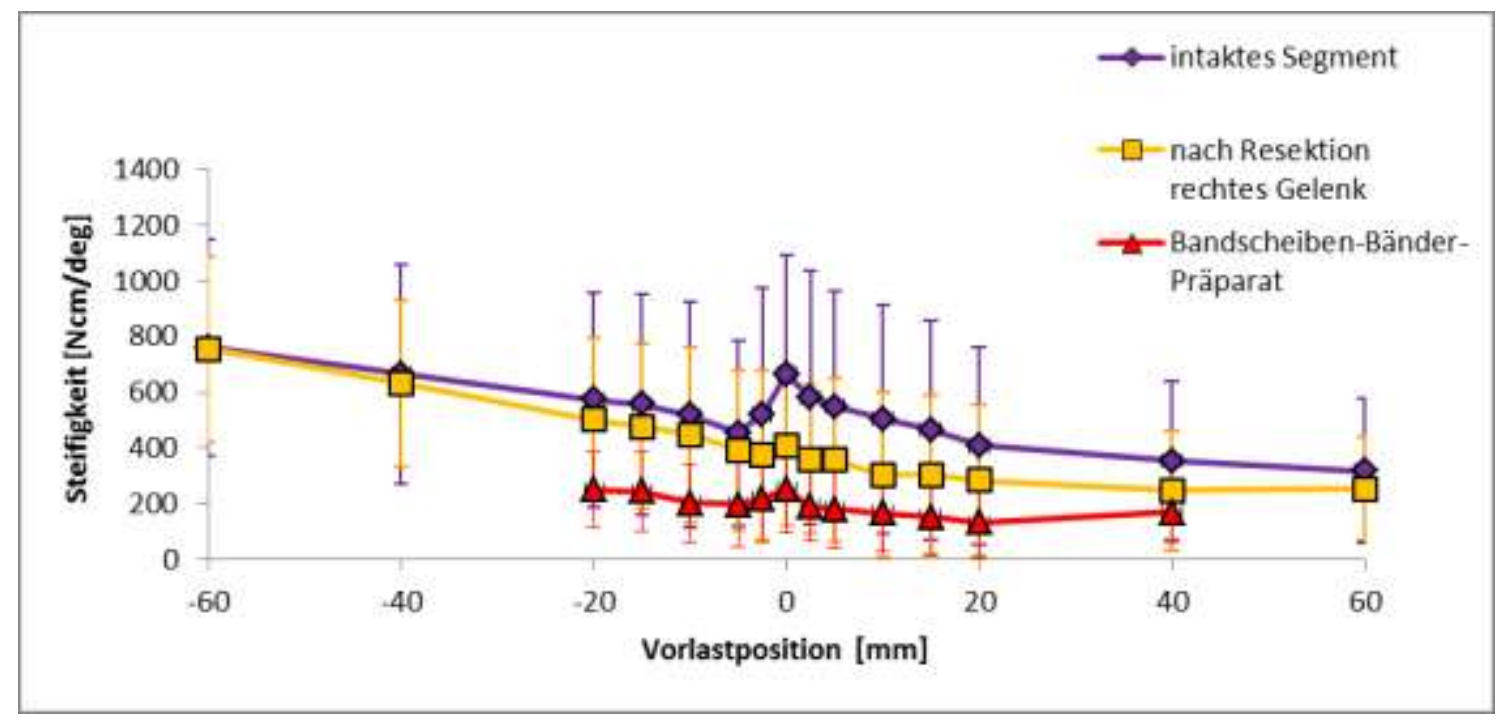

Abbildung 40: Mittlere minimale Steifigkeit in Ncm/deg bei allen gemessenen sagittalen Vorlastpositionen; Das intakte Segment wies einen Steifigkeitsverlust bei gering dorsalen Vorlastpositionen auf. Dieser Effekt war nach den Resektionen vermindert. Vorlast 200 N; alle Resektionszustände; Axialrotation 


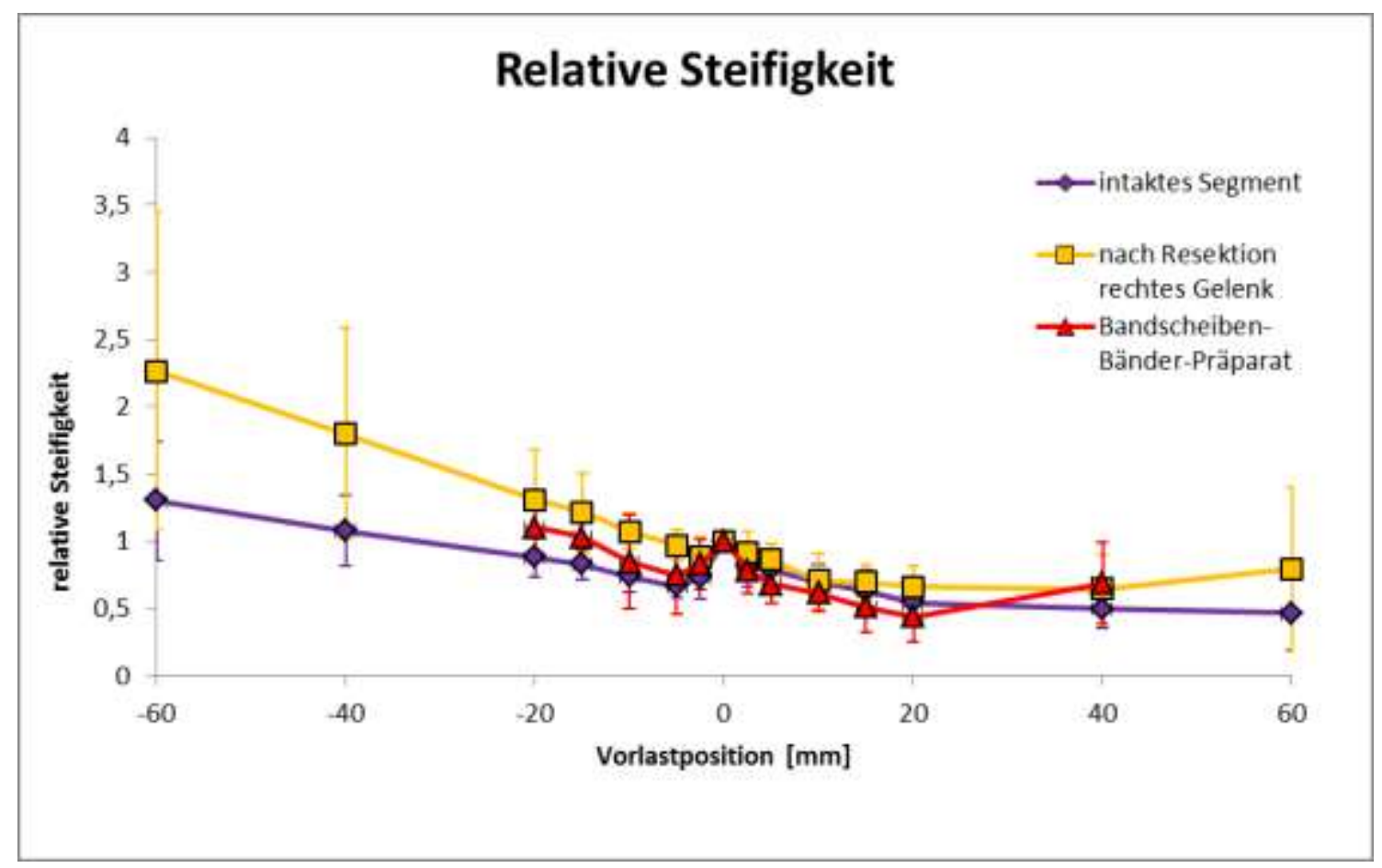

Abbildung 41: Mittlere relative Steifigkeit bei allen gemessenen sagittalen Vorlastpositionen. Das Absinken der Steifigkeit bei gering dorsalen Vorlastpositionen war beim Bandscheiben-Bänder-Präparat vermindert. Alle Resektionszustände; Axialrotation

Tabelle 8: Mittlere minimale Steifigkeit in Ncm absolut und in Prozent der Steifigkeit bei zentraler Vorlast bei verschiedenen sagittalen Vorlastpositionen; alle Segmentzustände; Standardabweichung in Klammern; Axialrotation

\begin{tabular}{|c|c|c|c|c|c|c|}
\hline $\begin{array}{l}\text { Segmentzustand/ } \\
\text { Vorlastposition }(\mathrm{mm})\end{array}$ & $\begin{array}{c}60 \\
\text { dorsal }\end{array}$ & $\begin{array}{c}10 \\
\text { dorsal }\end{array}$ & $\begin{array}{c}2,5 \\
\text { dorsal }\end{array}$ & zentral & $\begin{array}{c}20 \\
\text { ventral }\end{array}$ & $\begin{array}{c}60 \\
\text { ventral }\end{array}$ \\
\hline intaktes Segment & $\begin{array}{c}762( \pm 388) \\
130 \%\end{array}$ & $\begin{array}{c}518( \pm 405) \\
74 \%\end{array}$ & $\begin{array}{c}454( \pm 331) \\
73 \%\end{array}$ & $\begin{array}{c}664( \pm 428) \\
100 \%\end{array}$ & $\begin{array}{c}408( \pm 357) \\
54 \%\end{array}$ & $\begin{array}{c}318( \pm 260) \\
46 \%\end{array}$ \\
\hline $\begin{array}{l}\text { nach Resektion des } \\
\text { rechten Gelenks }\end{array}$ & $\begin{array}{c}755( \pm 331) \\
226 \%\end{array}$ & $\begin{array}{c}448( \pm 298) \\
107 \%\end{array}$ & $\begin{array}{c}374( \pm 304) \\
89 \%\end{array}$ & $\begin{array}{c}406( \pm 287) \\
100 \%\end{array}$ & $\begin{array}{c}284( \pm 271) \\
66 \%\end{array}$ & $\begin{array}{c}252( \pm 184) \\
79 \%\end{array}$ \\
\hline $\begin{array}{c}\text { Bandscheiben-Bänder- } \\
\text { Präparat }\end{array}$ & & $\begin{array}{c}203( \pm 141) \\
85 \%\end{array}$ & $\begin{array}{c}218( \pm 158) \\
83 \%\end{array}$ & $\begin{array}{c}253( \pm 155) \\
100 \%\end{array}$ & $\begin{array}{c}133( \pm 128) \\
44 \%\end{array}$ & \\
\hline
\end{tabular}

\subsubsection{Laterale Verschiebung der Vorlast}

\section{Intaktes Segment}

Die größte Steifigkeit fand sich bei zentraler Vorlastposition (664 Ncm/deg). Bei Verschiebung der Vorlast nach lateral kam es initial zu einem Absinken der Steifigkeit, danach blieb sie ungefähr auf einem Level (Abbildung 42). Die Werte von links- und rechts-lateraler Vorlast betrugen 530 bzw. 494 Ncm/deg. Das entspricht 80 \% bzw. $74 \%$ der Steifigkeit bei zentraler Vorlast. 


\section{Segment nach Resektion des rechten Zwischenwirbelgelenks}

Die Präparate wiesen bei zentraler Belastung mit 438 Ncm/deg die größte Steifigkeit auf. Eine Lateralverschiebung der Vorlast nach links bzw. rechts führte schon nach $5 \mathrm{~mm}$ zu einem deutlichen Sinken der mittleren Steifigkeit auf $398 \mathrm{Ncm} / \mathrm{deg}$ (= $91 \%$ ) bzw. 390 Ncm/deg (= 89 \%). Im Gegensatz zum intakten Segment blieben die Werte bei zusätzlicher Lateralisierung nicht konstant. Die durchschnittliche Steifigkeit bei maximaler Lateralisierung betrug $403 \mathrm{Ncm} / \mathrm{deg}$ bei linker und $433 \mathrm{Ncm} / \mathrm{deg}$ bei rechter Vorlast. Der Unterschied zwischen zentraler und lateraler Vorlast war geringer als vor der Resektion (Abbildung 42).

\section{Bandscheiben-Bänder-Präparat}

Die größte Steifigkeit fand sich wie auch vor den Resektionen bei zentraler Vorlastposition (253 Ncm/deg). Durch eine Lateralverschiebung um 10 mm konnten Veränderungen auf $170 \mathrm{Ncm} / \mathrm{deg}$ (links) bzw. $168 \mathrm{Ncm} / \mathrm{deg}$ (rechts) festgestellt werden. Laterale Vorlastpositionen von $20 \mathrm{~mm}$ führten zu einer Steifigkeit von je $178 \mathrm{Ncm} / \mathrm{deg}$ (Abbildung 42). Wie auch beim intakten Segment kam es durch leichte Lateralisierung zu einem Absinken der Steifigkeit auf etwa $67 \%$, im Laufe der Lateralverschiebung zu einer verhältnismäßig kleinen Zunahme auf $70 \%$.

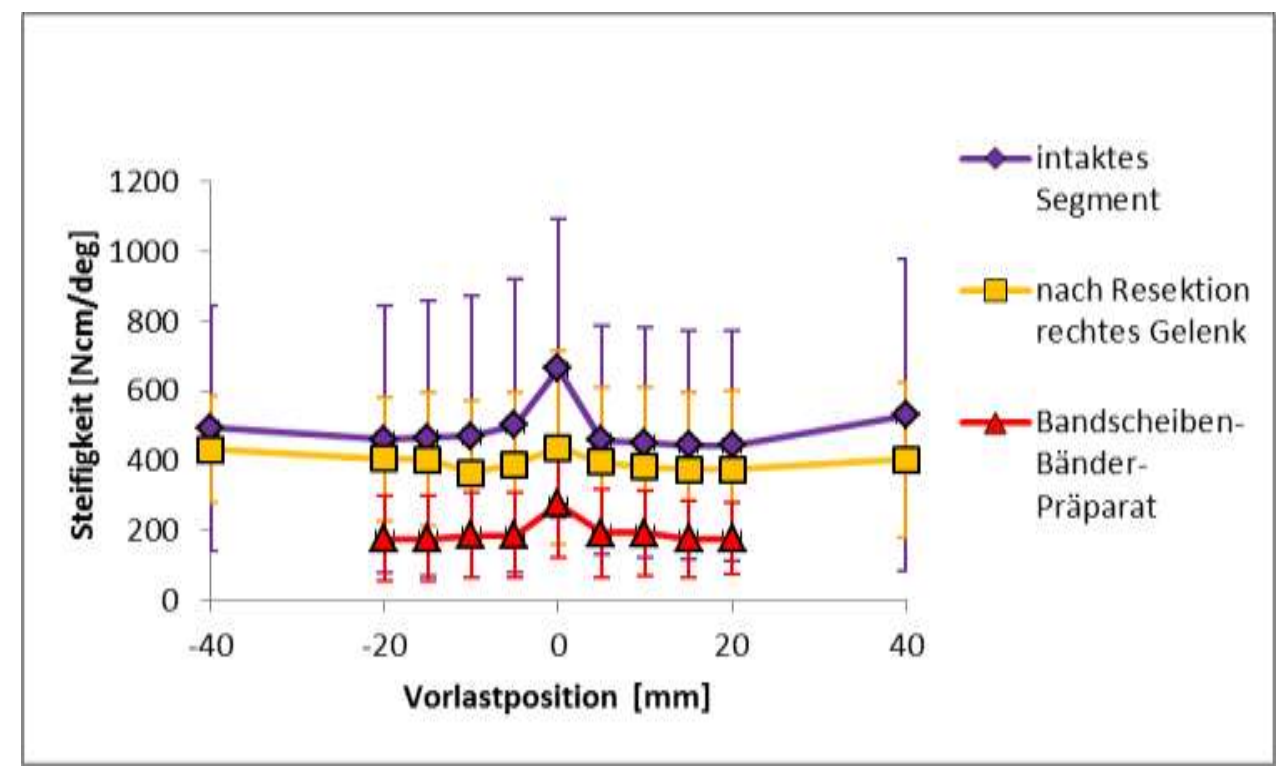

Abbildung 42: Mittlere minimale Steifigkeit in $\mathrm{Ncm} / \mathrm{deg}$ bei allen gemessenen lateralen Vorlastpositionen. Die Steifigkeit nahm unabhängig vom Resektionsstatus durch Lateralverschiebung ab. Vorlast $200 \mathrm{~N}$; alle Resektionszustände; Axialrotation

\subsubsection{Veränderung der Größe der Vorlast}

Unabhängig vom Resektionszustand führte eine Erhöhung der Vorlast zu einer Zunahme der Steifigkeit, eine Verringerung zur Abnahme. Dabei waren die Veränderungen besonders nach der Resektion des rechten Bogengelenks sichtbar. Sie lagen bei 157 \% bzw. 68 \% des Ausgangswertes (200 N). Im intakten Segment und 
Bandscheiben-Bänder-Präparat führte hingegen die Vergrößerung der Vorlast zu geringerer Variation (103\% bzw.113\%).

\subsubsection{Zusammenfassung der Ergebnisse: „Axialrotation“}

Tabelle 9: Zusammenfassung der Ergebnisse des Experiments "Axialrotation"

\section{Lage und Wanderung der Schraubachsen}

\begin{tabular}{|c|c|l|}
\hline \multirow{4}{*}{ intaktes Segment } & $\begin{array}{c}\text { VL } \\
\text { zentral }\end{array}$ & $\begin{array}{l}\text { Rastpolkurve ist dreieckförmig zwischen dorsaler Bandscheibenkante und } \\
\text { Bandscheibenmitte }\end{array}$ \\
\cline { 2 - 3 } & $\begin{array}{c}\text { VL } \\
\text { ventral }\end{array}$ & $\begin{array}{l}\text { Rastpolkurve verläuft wie ein Dreieck ohne Basislinie. Die Spitze liegt in der } \\
\text { dorsalen Bandscheibe, die Schenkel wandern bis zu den Gelenken }\end{array}$ \\
\cline { 2 - 3 } & $\begin{array}{c}\mathrm{VL} \\
\text { dorsal }\end{array}$ & Rastpolkurve ist hufeisenförmig und liegt in der dorsalen Bandscheibenhälfte \\
\hline \multirow{2}{*}{$\begin{array}{c}\text { nach Resektion } \\
\text { des rechten } \\
\text { Gelenks }\end{array}$} & $\begin{array}{c}\text { VL } \\
\text { zentral } \\
\text { ventral }\end{array}$ & $\begin{array}{l}\text { Rastpolkurve verläuft bei } \alpha<0^{\circ} \text { wie intaktes Segment, bei } \alpha>0^{\circ} \text { im Bogen } \\
\text { nach links-dorsal; Kurve liegt insgesamt in der linken Bandscheibenhälfte }\end{array}$ \\
\cline { 2 - 3 } & $\begin{array}{c}\text { VL } \\
\text { dorsal }\end{array}$ & $\begin{array}{l}\text { Rastpolkurve verläuft zwischen Bandscheibe und verbliebenem Gelenk } \\
\text { linkem Bandscheibendrittel und Gelenk }\end{array}$ \\
\hline $\begin{array}{c}\text { Bandscheiben- } \\
\text { Bänder-Präparat }\end{array}$ & \multicolumn{2}{|c|}{$\begin{array}{l}\text { Rastpolkurven liegen unabhängig von der Vorlastposition nahezu ortsfest in } \\
\text { der Bandscheibe }\end{array}$} \\
\hline
\end{tabular}

\section{Ausrichtung der Schraubachse}

\begin{tabular}{|c|c|c|}
\hline \multirow{3}{*}{ intaktes Segment } & $\begin{array}{c}\mathrm{VL} \\
\text { zentral }\end{array}$ & $\begin{array}{l}\text { Neigung dezent nach ventral und zusätzlich in Rotationsmaxima nach lateral } \\
\left(\alpha<0^{\circ}: \text { Rechtsneigung; } \alpha>0^{\circ}: \text { Linksneigung) }\right.\end{array}$ \\
\hline & $\begin{array}{c}\mathrm{VL} \\
\text { ventral }\end{array}$ & $\begin{array}{l}\text { Neigung nach dorsal und zusätzlich in Rotationsmaxima lateral, entgegen- } \\
\text { gesetzt zu zentraler } \mathrm{VL}\left(\alpha<0^{\circ} \text { : Linksneigung; } \alpha>0^{\circ} \text { : Rechtsneigung) }\right.\end{array}$ \\
\hline & $\begin{array}{c}\mathrm{VL} \\
\text { dorsal }\end{array}$ & Neigung ähnlich wie bei zentraler VL \\
\hline \multirow{3}{*}{$\begin{array}{l}\text { nach Resektion } \\
\text { des rechten Ge- } \\
\text { lenks }\end{array}$} & $\begin{array}{c}\mathrm{VL} \\
\text { zentral }\end{array}$ & Neigung ähnlich wie bei intaktem Segment \\
\hline & $\begin{array}{c}\mathrm{VL} \\
\text { ventral }\end{array}$ & Neigung ähnlich wie bei intaktem Segment \\
\hline & $\begin{array}{c}\mathrm{VL} \\
\text { dorsal }\end{array}$ & $\begin{array}{l}\text { Neigung ähnlich wie bei intaktem Segment; in Rechtsrotation verringerter } \\
\text { Neigungswinkel }\end{array}$ \\
\hline $\begin{array}{l}\text { Bandscheiben- } \\
\text { Bänder-Präparat }\end{array}$ & & $\begin{array}{l}\text { sagittale Achsabweichung in Rotationsmaxima verringert; laterale Abwei- } \\
\text { chung tendenziell wie bei intaktem Segment bei insgesamt verringertem } \\
\text { Neigungswinkel }\end{array}$ \\
\hline
\end{tabular}

\section{Steifigkeit}

\begin{tabular}{|c|l|}
\hline intaktes Segment & $\begin{array}{l}\text { Abfall der Steifigkeit bei gering dorsaler Vorlast auf } 73 \% \text {; bei weiterer Dor- } \\
\text { salverschiebung sukzessiver Anstieg auf } 130 \% \text {; bei ventraler VL sukzessi- } \\
\text { ver Abfall der Steifigkeit auf } 46 \%\end{array}$ \\
\hline $\begin{array}{c}\text { nach Resektion des rechten } \\
\text { Gelenks }\end{array}$ & $\begin{array}{l}\text { Abfall der Steifigkeit bei gering dorsaler Vorlast auf } 89 \% ; \text { bei weiterer Dor- } \\
\text { salverschiebung Anstieg auf } 226 \%\end{array}$ \\
\hline $\begin{array}{c}\text { Bandscheiben-Bänder- } \\
\text { Präparat }\end{array}$ & $\begin{array}{l}\text { Abfall der Steifigkeit bei gering dorsaler Vorlast auf } 83 \% \text {; vorlastabhängige } \\
\text { Entwicklung der Steifigkeit vergleichbar mit intaktem Segment; insgesamt } \\
\text { verminderte Steifigkeit }\end{array}$ \\
\hline
\end{tabular}




\subsection{Ventralflexion-Dorsalextension}

\subsubsection{Rastpolkurven}

In der Ventralflexions-Dorsalextensionsbewegung lag die momentane Schraubachse (IHA) nahezu parallel zur y-Achse des Koordinatensystems. Daher konnte die Wanderung der IHA dargestellt werden, indem die Schnittpunkte der Schraubachsen mit der x-z-Ebene des Koordinatensystems aufgezeichnet wurden. Diese Rastpolkurve liegt so in der median-sagittalen Schnittebene. Ihre Einordnung in das Segment ist durch die Projektion des CT-Bildes veranschaulicht.

\subsubsection{Veränderung der Größe der Vorlast}

Die Erhöhung der Vorlast, deren Wirkungslinie durch das Zentrum des Wirbelkanals lief, führte zu einer Verlagerung der Rastpolkurve nach dorso-kaudal. Nach den einzelnen Resektionen wurde das Ausmaß der Verlagerung dezent größer (Abbildung 43).

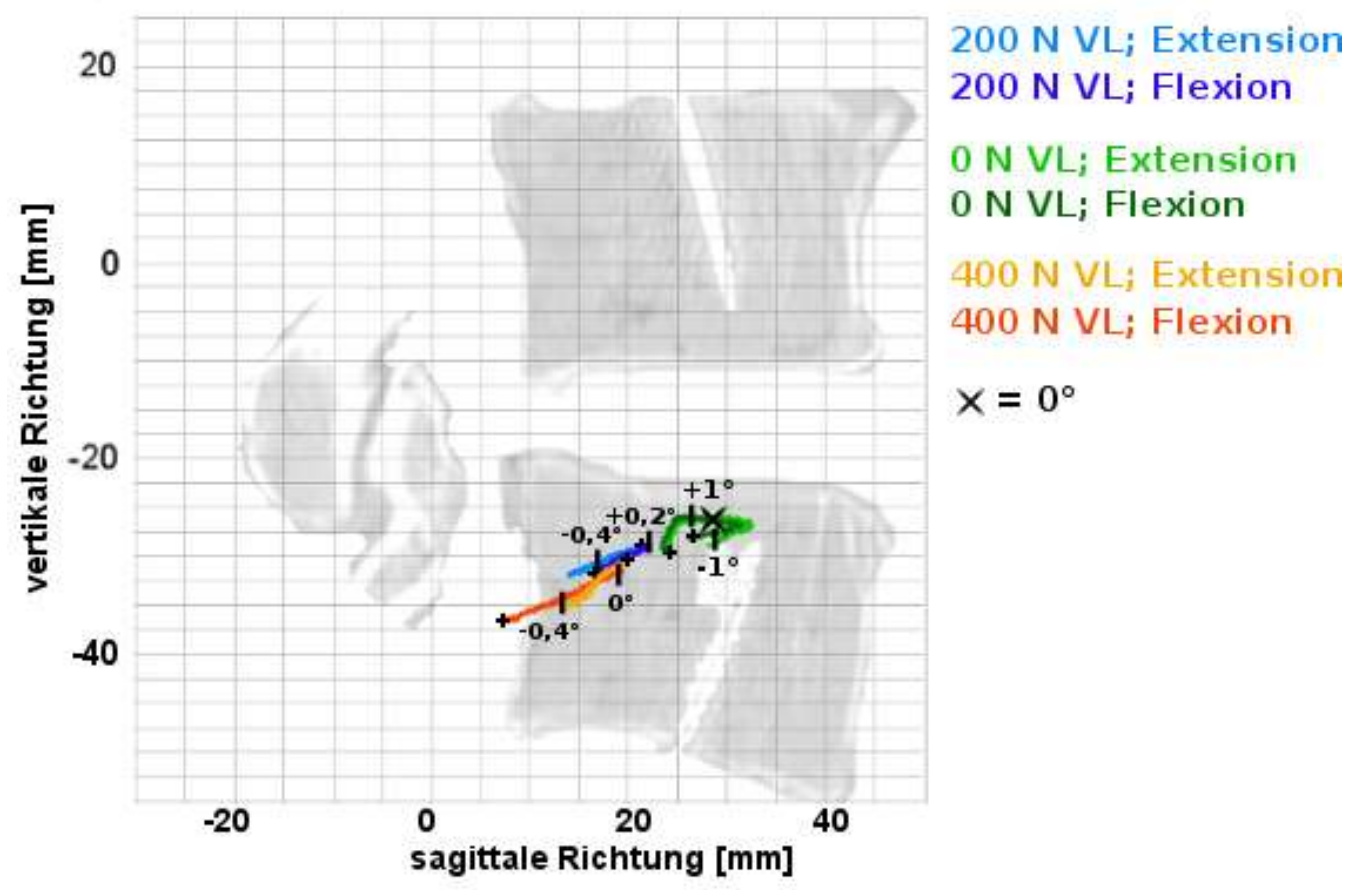

Abbildung 43: Rastpolkurven des Präparats D bei unterschiedlichen Größen der Vorlast. Eine Verringerung der Vorlastgröße ging mit einer Verschiebung der IHA nach ventral und kranial einher, eine Erhöhung mit einer Verschiebung nach dorsal und kaudal. Z.n. Resektion des rechten Wirbelbogengelenks; FlexionExtension; die Lage des Wirbelbogengelenks ist eingeblendet; + markiert den Bewegungsbeginn; die Markierungen mit Zahlen beschreiben das Rotationsausmaß. 


\subsubsection{Sagittale Verschiebung der Vorlast}

\section{Intaktes Segment}

Die Kurve des Beispielpräparats $D$ bewegte sich bei zentraler Vorlast beginnend in Ventralflexion bis zu einer Dorsalneigung von etwa $0,8^{\circ}$ um maximal $2 \mathrm{~mm}$ nach dorsokaudal, bei größeren Drehwinkeln zügig um mehr als $10 \mathrm{~mm}$ nach dorsal bis in den Wirbelkanal hinein (Abbildung 44). Die Rastpolkurven der anderen Segmente lagen größtenteils in etwa parallel zur oberen Deckplatte des unteren Wirbelkörpers und wenige Millimeter bis ca. $1 \mathrm{~cm}$ kaudal dieser Deckplatte.

War die Vorlast ventral positioniert, lag die Kurve jeweils etwa $3 \mathrm{~mm}$ weiter ventral und kranial, also etwa mittig auf Höhe der oberen Deckplatte des unteren Wirbelkörpers. Die maximale Wanderung nach dorso-kaudal betrug nun während der gesamten Bewegung $7 \mathrm{~mm}$.

Bei dorsaler Vorlast war die Kurve im Vergleich zur der bei zentraler Vorlast nach dorso-kaudal verschoben. Diese Verschiebung betrug im Punkt der maximalen Flexion $8 \mathrm{~mm}$ nach dorsal und $4 \mathrm{~mm}$ nach kaudal. Zusätzlich kam es im Verlauf der Dorsalneigung zu einer gleichmäßigen Wanderung der Schraubachsen um 12 mm in Richtung Wirbelkanal.

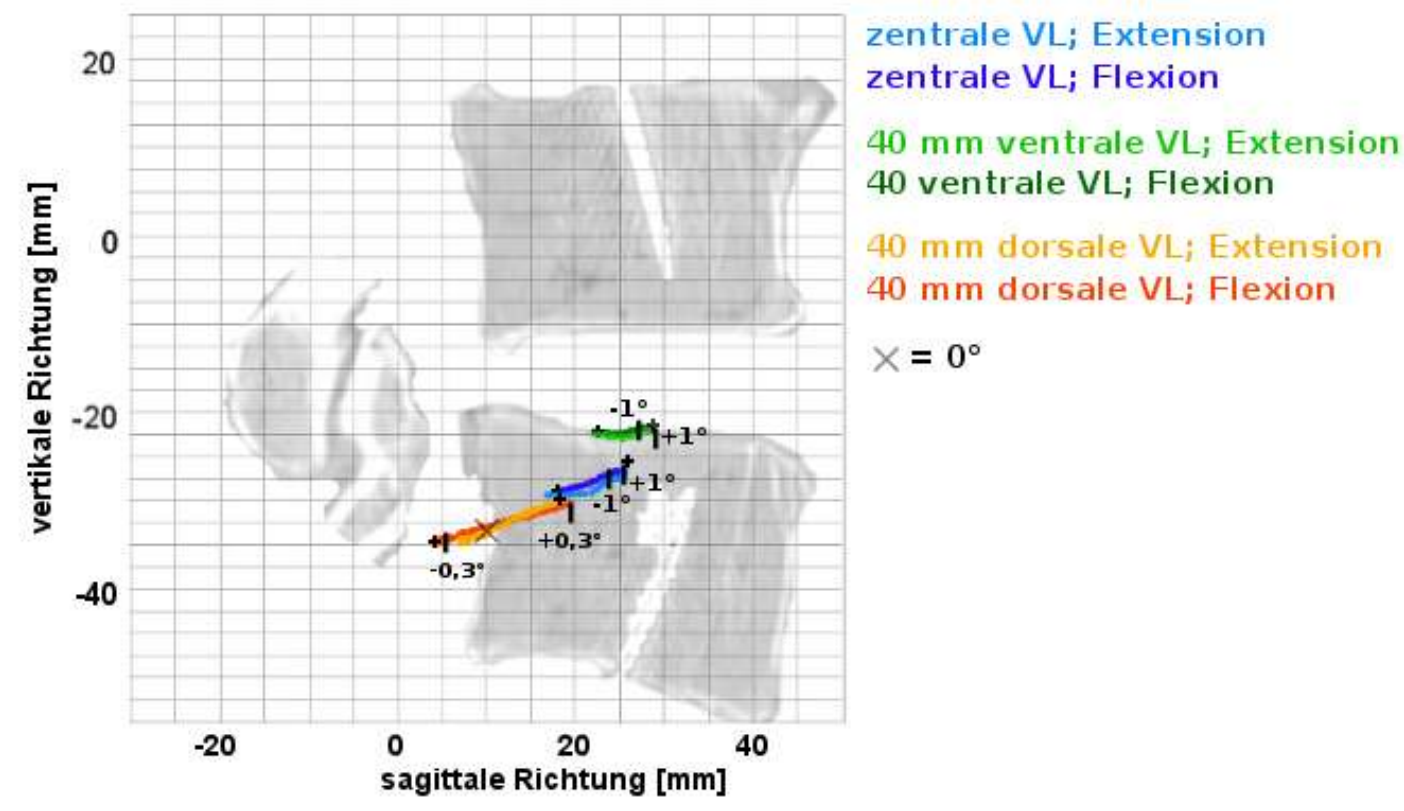

Abbildung 44: Rastpolkurven des intakten Präparats D bei unterschiedlichen sagittalen Vorlastpositionen. Ventrale Vorlasten gingen mit einer Verschiebung der IHA nach ventral und kranial einher, dorsale Vorlasten mit einer Verschiebung nach dorsal und kaudal. Vorlast $200 \mathrm{~N}$; Flexion-Extension; die Lage der Wirbelbogengelenke ist eingeblendet; + markiert den Bewegungsbeginn; die Markierungen mit Zahlen beschreiben das Rotationsausmaß. 


\section{Segment nach Resektion des rechten Zwischenwirbelgelenks}

Bei zentraler Vorlast kam es im Vergleich zum intakten Segment zu einer Verlagerung der Kurve nach dorso-kaudal (4,5 bzw. 3,5 mm). Die Wanderung verlief nun gleichmäBig bis zu einer Strecke von $8 \mathrm{~mm}$.

Wurde die Vorlast ventral positioniert verlagerte sich die Rastpolkurve $8,5 \mathrm{~mm}$ bzw. $6 \mathrm{~mm}$ weiter nach kranial bzw. ventral. Die Bewegung verlief im Gegensatz zum intakten Segment nahezu ortsfest. Die Gesamtwanderung betrug $2 \mathrm{~mm}$ gegenüber $7 \mathrm{~mm}$ vor der Resektion. Die Position der Kurve blieb jedoch vergleichbar.

Im Gegensatz zum intakten Segment führte eine Verschiebung der Vorlast nach dorsal nicht zu homogenen Positionsänderungen der Schraubachsen. Zwar kam es bei den meisten Präparaten zu einer Verlagerung der Rastpolkurve nach dorsokaudal, jedoch war diese Verschiebung unterschiedlich stark ausgeprägt. Die Wanderung nach dorsal im Extensionsverlauf war vermindert, während die Kaudalkomponente verschwand bzw. nur gering ausgeprägt war (Abbildung 45).

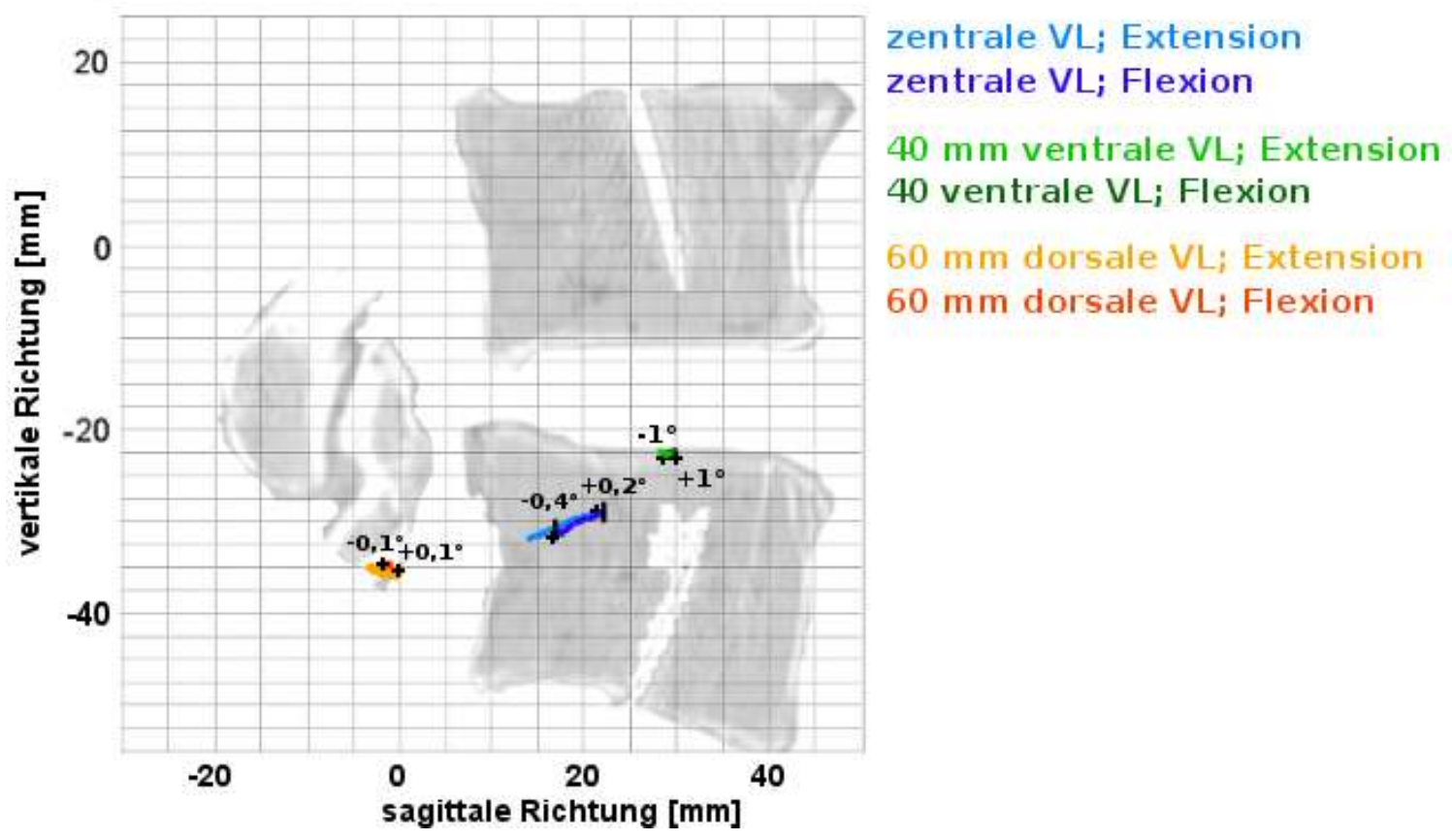

Abbildung 45: Rastpolkurven des Präparats D nach Resektion des rechten Wirbelbogengelenks bei unterschiedlichen sagittalen Vorlastpositionen. Eine Verlagerung der Vorlast führte zu ortsfesten IHA. Vorlast $200 \mathrm{~N}$; Flexion-Extension; die Lage des Wirbelbogengelenks ist eingeblendet; + markiert den Bewegungsbeginn; die Markierungen mit Zahlen beschreiben das Rotationsausmaß.

\section{Bandscheiben-Bänder-Präparat}

Bei zentraler Vorlastposition verlief die Kurve den Großteil der Bewegung ortsfest an der oberen Deckplatte und etwas dorsal der Mittellinie des unteren Wirbelkörpers. Am Beispielpräparat $D$ wanderte sie ab einer Dorsalextension von etwa $0,6^{\circ}$ nach dorsal. Die Bewegungsstruktur glich somit der des intakten Segments, der Kurvenbeginn lag nun jedoch etwa $2 \mathrm{~mm}$ weiter kranial und $4 \mathrm{~mm}$ weiter dorsal (Abbildung 46). 
Die Schraubachsen bei ventraler Vorlast verblieben nach den Resektionen stärker ortsfest. Die Rastpolkurve lag in Extension etwa mittig im unteren Bandscheibensegment. Während der Extensionsbewegung beschrieb die Schraubachse eine geringe dorsal gerichtete Wanderung über $4 \mathrm{~mm}$. Die Schraubachsen waren um $8 \mathrm{~mm}$ nach ventral und 3,5 mm nach kranial verlagert. Das Ende der Rastpolkurve in Flexionsstellung lag $1,5 \mathrm{~mm}$ weiter ventral und 2,5 mm weiter kranial als im intakten Segment.

Auch nach Resektion beider Zwischenwirbelgelenke gingen dorsale Vorlastpositionen mit einer Verlagerung der Rastpolkurve nach dorso-kaudal einher. Sie verlief in gleichmäßigerer Geschwindigkeit und über eine Länge von ca. $8 \mathrm{~mm}$, wobei die kaudale Wanderkomponente ausgeprägter war als im intakten Segment. Der Kurvenverlauf begann ungefähr $4 \mathrm{~mm}$ nach dorsal und $1 \mathrm{~mm}$ nach kaudal verlagert. Verglichen mit dem unresezierten Präparat war der Kurvenverlauf einige Millimeter kürzer und nach kranial versetzt $(5 \mathrm{~mm})$.

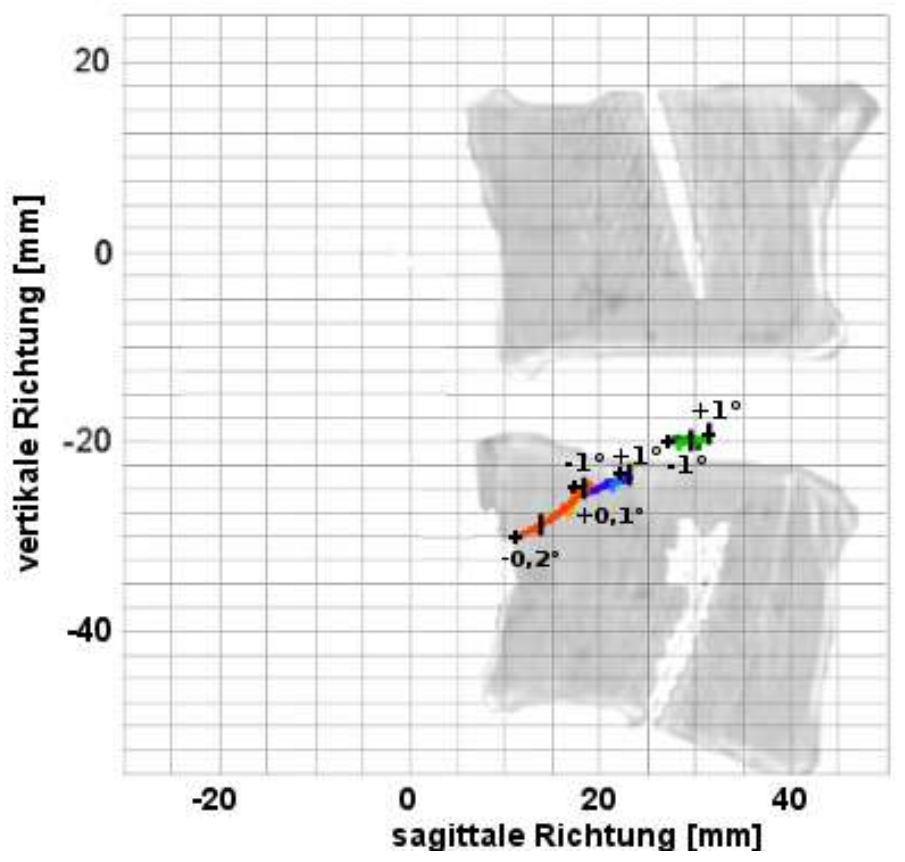

zentrale VL; Extension zentrale VL; Flexion

$40 \mathrm{~mm}$ ventrale VL; Extension 40 ventrale VL; Flexion

$15 \mathrm{~mm}$ dorsale VL; Extension $15 \mathrm{~mm}$ dorsale VL; Flexion

Abbildung 46: Rastpolkurven des Bandscheiben-Bänder-Präparats D bei unterschiedlichen sagittalen Vorlastpositionen. Auch nach der Resektion ging die Verschiebung der Vorlast mit einer Verlagerung der IHA einher. Vorlast $200 \mathrm{~N}$; Flexion-Extension; + markiert den Bewegungsbeginn; die Markierungen mit Zahlen beschreiben das Rotationsausmaß.

\subsubsection{Laterale Verschiebung der Vorlast}

Im intakten Segment lagen die Rastpolkurven wie auch bei zentraler Vorlastposition einige Millimeter unterhalb der oberen Deckplatte des unteren Wirbelkörpers (Abbildung 47). Sie verliefen jedoch den Großteil der Bewegung $\left(2,5--0,5^{\circ}\right)$ zentrierter und wanderten im Extensionsverlauf erst ab $-0,5^{\circ}$ nach dorsal. Es konnten keine wesentlichen Unterschiede zwischen rechten und linken Vorlastpositionen festgestellt werden. 


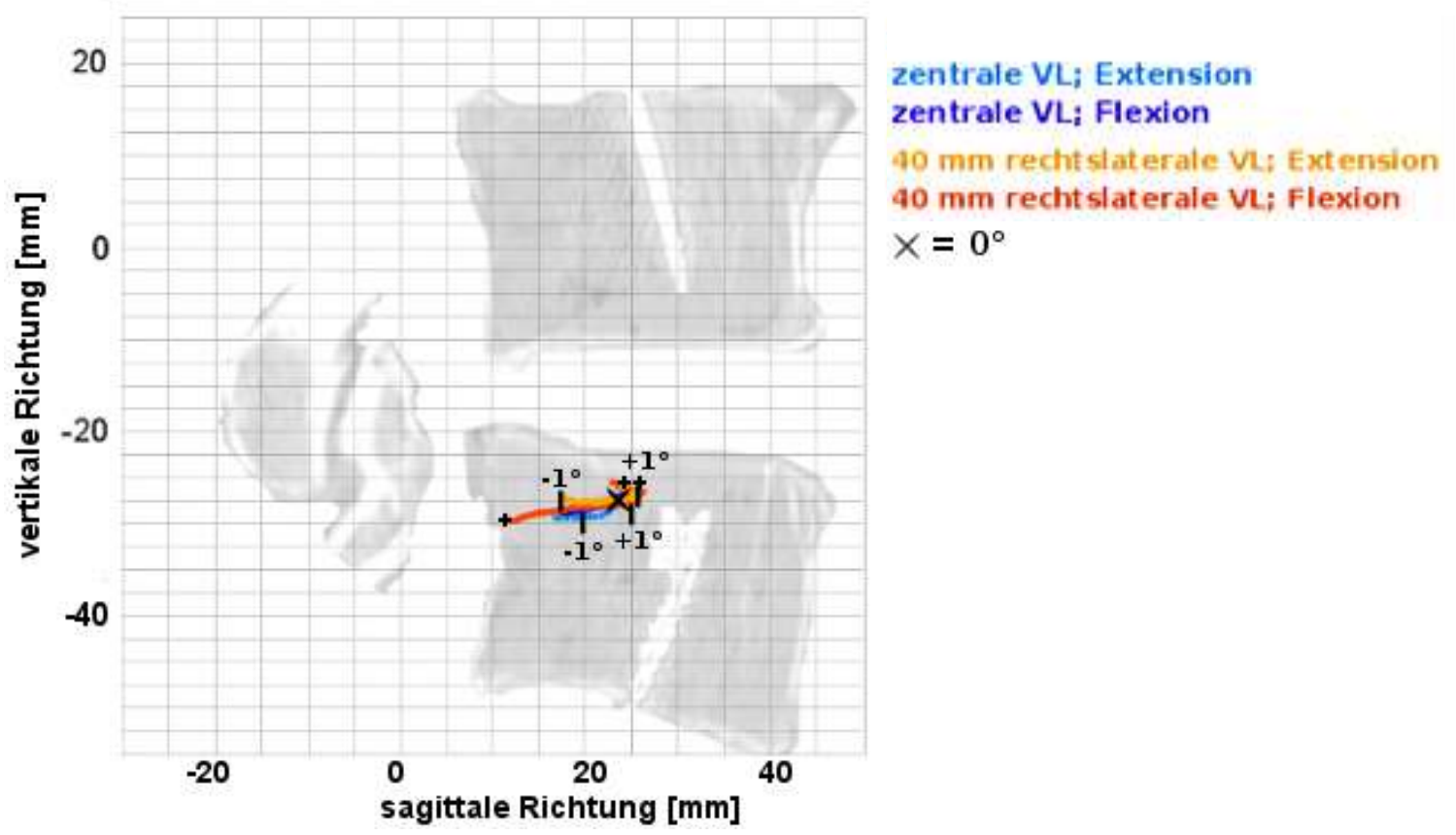

Abbildung 47: ähnlich verlaufende Rastpolkurven des intakten Präparats $D$ bei zentraler und rechtslateraler Vorlastposition. Vorlast $200 \mathrm{~N}$; Flexion-Extension; die Lage der Wirbelbogengelenke ist eingeblendet; + markiert den Bewegungsbeginn; die Markierungen mit Zahlen beschreiben das Rotationsausmaß.

Nach der Resektion des rechten Gelenks waren die Rastpolkurven bei linksseitigen Vorlastpositionen ähnlich komprimiert wie die des intakten Segments. Rechte Vorlasten führten in Bezug auf die pro Rotationswinkel zurückgelegte Wanderung zu einem gleichmäßigeren Verlauf. Die Position hingegen war besonders bei linken Vorlasten um $5 \mathrm{~mm}$ bei Präparat $\mathrm{D}$ nach ventral verlagert (Abbildung 48). 


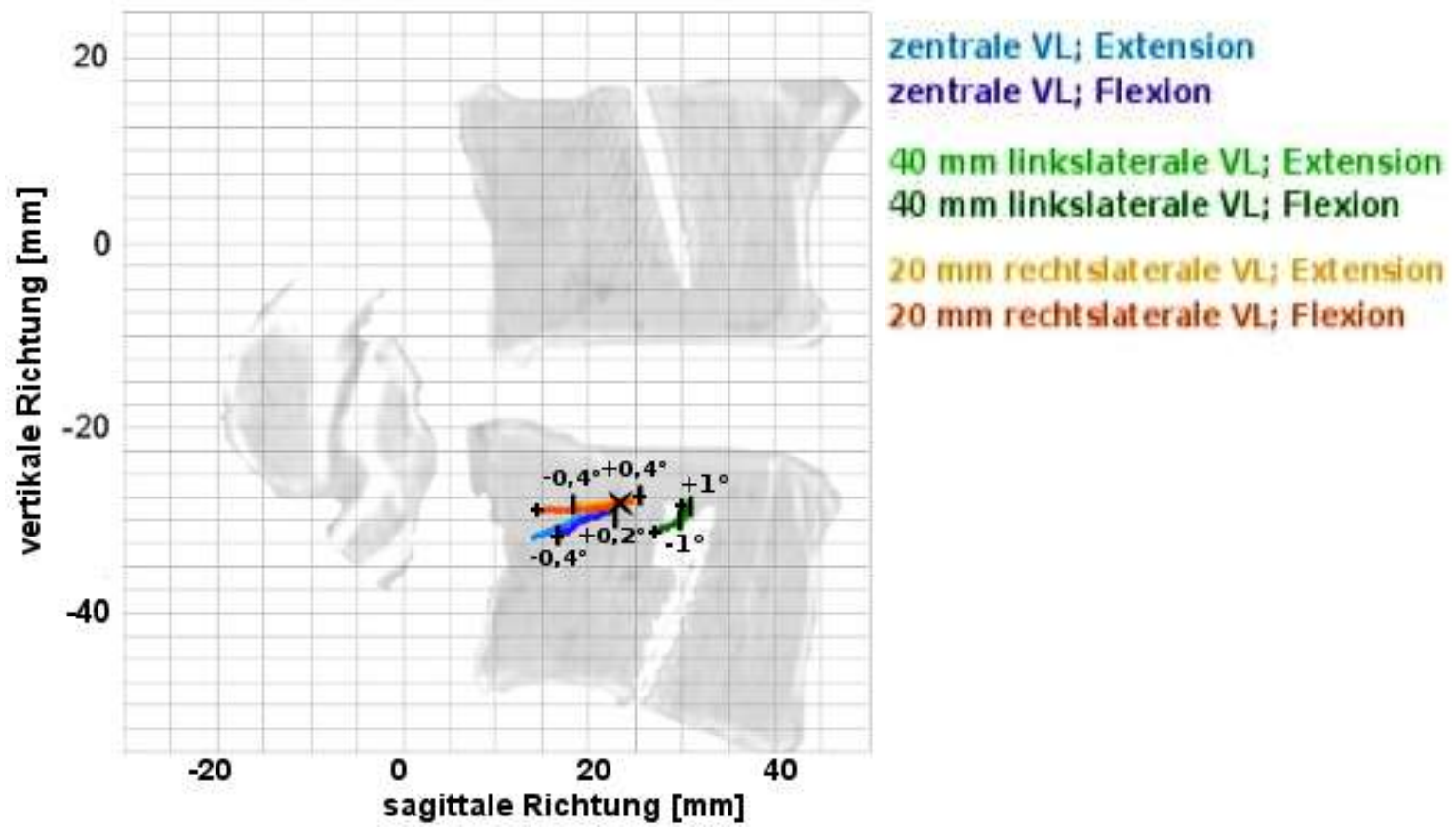

Abbildung 48: Rastpolkurven des Präparats D nach Resektion des rechten Wirbelbogengelenks bei unterschiedlichen lateralen Vorlastpositionen. Links-laterale Vorlasten führten zu einer Ventralverlagerung der IHA mit kürzerer Wanderstrecke. Nach rechts-lateraler Positionierung der Vorlast zeigten sich nur geringfügige Veränderungen des Kurvenverlaufs. Vorlast $200 \mathrm{~N}$; Flexion-Extension; die Lage des Wirbelbogengelenks ist eingeblendet; + markiert den Bewegungsbeginn; die Markierungen mit Zahlen beschreiben das Rotationsausmaß.

Am Bandscheiben-Bänder-Präparat (Abbildung 49) führten rechte und linke Vorlastpositionen zu einem vergleichbaren Kurvenverlauf und -position. Die Rastpole verlagerten sich durch Lateralisierung um $3 \mathrm{~mm}$ nach dorsal, der Kurvenverlauf war verlängert. Im Vergleich zum intakten Segment waren die Kurven nun $7 \mathrm{~mm}$ nach dorsal und 3,5 mm nach kranial versetzt. 


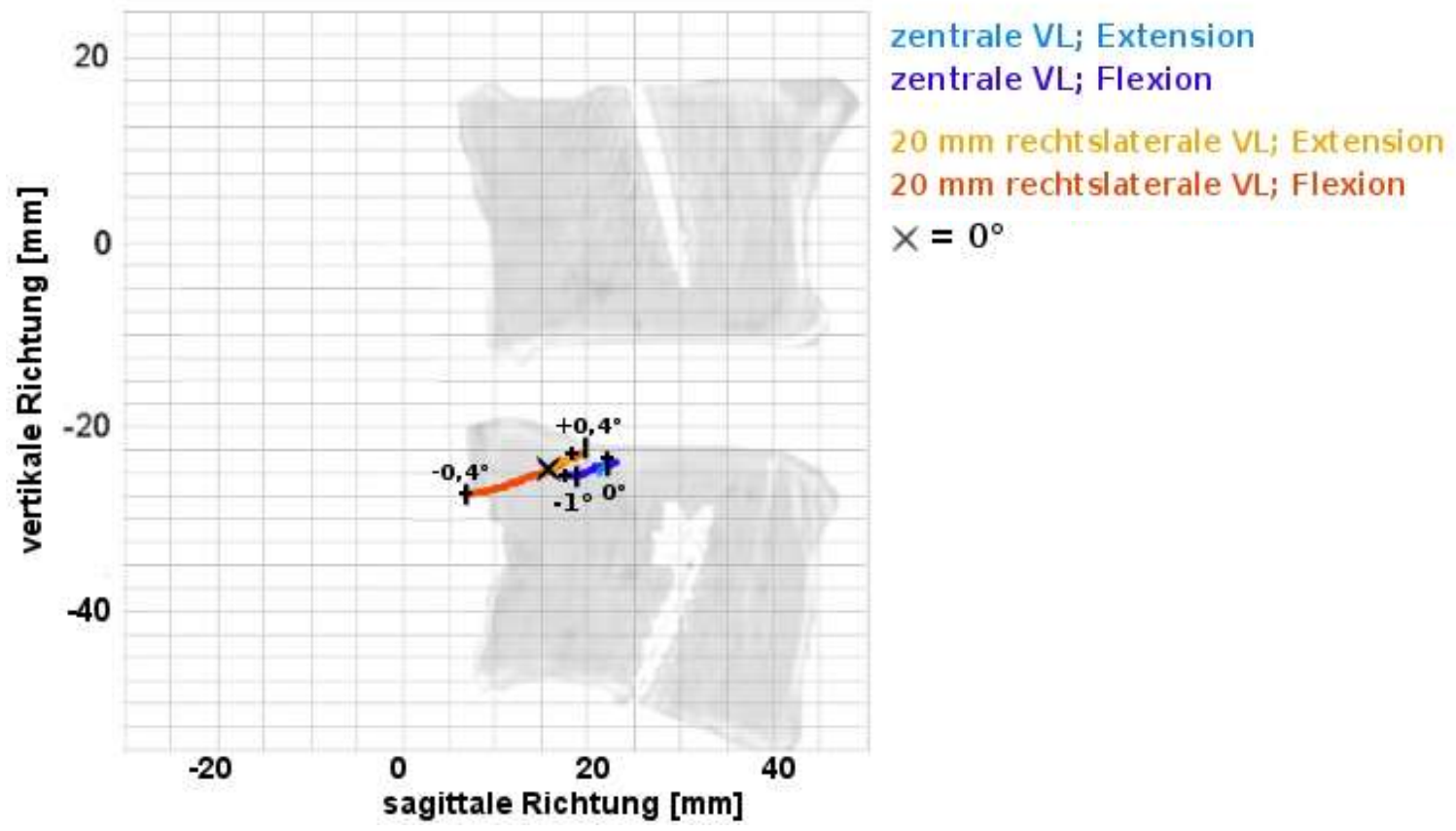

Abbildung 49: Rastpolkurven des Bandscheiben-Bänder-Präparats $D$ bei unterschiedlichen lateralen Vorlastpositionen. Laterale Vorlastpositionen führten zu einem verlängerten Kurvenverlauf. Vorlast $200 \mathrm{~N}$; Flexion-Extension; + markiert den Bewegungsbeginn; die Markierungen mit Zahlen beschreiben das Rotationsausmaß.

\subsubsection{Ausrichtung der Schraubachsen}

Da es sich bei der Flexion-Extension um eine Bewegung nach Einwirkung eines Drehmoments in y-Richtung handelt, wird in diesem Abschnitt auch die Abweichung der momentanen Schraubachsen von einer Parallelen der y-Achse beschrieben. Die Richtungsangaben beziehen sich auf die positiven Bereiche des kartesischen Koordinatensystems („links“ und „kranial“). D.h. bei „ventraler Neigung“ verläuft die Schraubachse von links-ventral nach rechts-kaudal und umgekehrt. Entsprechend bedeutet eine „kraniale Neigung" eine von links-kranial nach rechts-dorsal verlaufende Achse und vice versa.

\subsubsection{Sagittale Verschiebung der Vorlast}

\section{Intaktes Segment}

Die Schraubachsen bei zentraler Vorlastposition waren während des gesamten Rotationsverlaufs nach ventral sowie minimal nach kaudal gekippt. Die stärkste Ventralneigung zeigte sich mit $4,34^{\circ}$ in Neutralstellung $\left(\alpha=0^{\circ}\right)$. In Dorsalextension war der Neigungswinkel auf $3,8^{\circ}$, in Ventralflexion auf $0,98^{\circ}$ verringert (Tabelle 10 ). Die kaudale Verkippung war generell gering und mit $1,28^{\circ}$ in Extensionsstellung am ausgeprägtesten (Tabelle 11).

Bei ventral positionierter Vorlast war die Schraubachse in Extension um 1,36 ${ }^{\circ}$ nach ventral geneigt (Tabelle 10). Während der Flexionsbewegung kippte sie stetig nach dorsal, so dass bei $\alpha=0^{\circ}$ eine Dorsalneigung von $0,1^{\circ}$, in Flexionsstellung von $3,08^{\circ}$ vorlag. Somit lagen die Schraubachsen während der gesamten Bewegung dorsaler als 
bei zentraler Vorlast. Des Weiteren fand eine Verkippung der Schraubachsen von $1,26^{\circ}$ nach kaudal (Extension) und bis $0,58^{\circ}$ nach kranial (Flexion) statt (Tabelle 11).

Wurde die Vorlast in dorsale Positionen verschoben war die Schraubachse permanent nach ventral geneigt. Die stärkste Neigung fand sich im Unterschied zur zentralen Vorlast nun bei Dorsalextension $\left(9,12^{\circ}\right)$. Bei $\alpha=0^{\circ}$ verringerte sie sich auf $5,38^{\circ}$, in Flexionsstellung auf $1,36^{\circ}$ (Tabelle 10). In vertikaler Richtung waren die Achsen im Flexionsverlauf zunehmend nach kaudal gekippt. Dabei wurden Winkel von 0,7 $7^{\circ}$ in Extension, $2,86^{\circ}$ bei $\alpha=0^{\circ}$ und $3,92^{\circ}$ in Flexion erreicht (Tabelle 11 ).

\section{Nach Resektion des rechten Zwischenwirbelgelenks}

Zentral platzierte Vorlasten verstärkten die Dorsalneigung in Extension auf $12^{\circ}$, während sie in Flexion auf 6,64 abgeschwächt war (Tabelle 10). Die Kaudalneigung lag nur noch in Extension vor $\left(6,26^{\circ}\right)$, in Neutral- bzw. Flexionsstellung kippten die Schraubachsen um $3,34^{\circ}$ bzw. $2,54^{\circ}$ nach kranial (Tabelle 11). Verglichen mit dem intakten Segment, bei dem die maximale Dorsalneigung in Flexion stattgefunden hatte, lag demnach eine Umkehr der Neigungstendenz in sagittaler Ausrichtung vor.

In ventraler Vorlastposition waren die momentanen Schraubachsen im Gegensatz zum intakten Segment während des gesamten Bewegungsablaufs nach dorsal gekippt. Das Maximum der Kippung fand sich dabei mit 10,24 in Flexionsstellung, das Minimum mit $3,44^{\circ}$ in Extension (Tabelle 10). Bei $\alpha=0^{\circ}$ lag eine Dorsalneigung von $9,02^{\circ}$ vor. Die Abweichung in der Vertikalen blieb minimal, während sie vor den Resektionen nach kaudal geneigt gewesen waren (Tabelle 11).

War die Vorlast dorsal positioniert, neigten sich die Schraubachsen ähnlich wie bei ventraler Vorlast in Extension am stärksten nach dorsal. Allerdings war der Neigungswinkel mit 5,9 $9^{\circ}$ deutlich geringer (Tabelle 10). In Neutralstellung betrug die Neigung noch $3,24^{\circ}$, in Flexion lag eine Ventralneigung von $3,9^{\circ}$ vor. Auch hier war die Neigungstendenz verglichen mit dem intakten Segment umgekehrt. Die permanente Kaudalkippung (Tabelle 11) war wie auch vor der Resektion in Flexion am stärksten $\left(5,4^{\circ}\right)$.

\section{Bandscheiben-Bänder-Präparat}

Eine zentral gelegene Vorlast resultierte in einer Verstärkung der Ventralneigung. Sie lag nun zwischen $5,18^{\circ}$ in Flexion und 8,5 in Extension (Tabelle 10). Es zeigte sich im Vergleich zum intakten Segment also eine Umkehr der Richtungstendenz bei $\alpha=0^{\circ}$ und Flexion. Die Kaudalkippung blieb vergleichbar mit der bei zentraler Vorlast. Im unresezierten Zustand war sie nur bei $\alpha=0^{\circ}$ deutlich ausgeprägter gewesen (Tabelle 11).

Bei ventral verschobener Vorlastposition waren die Achsen während der gesamten Bewegung nach ventral und minimal nach kranial gekippt. Die größte Ventralneigung fand sich mit $6^{\circ}$ in Flexionsstellung, die geringste mit 2,25 $5^{\circ}$ in Neutralstellung (Tabelle 10). Das Ausmaß der Kranialkippung war vergleichbar mit der Kaudalneigung des intakten Segments (Tabelle 11). In der sagittalen Achse hingegen hatte es nach den Resektionen einen Wechsel der maximalen Ventralkippung von der neutralen zur Ex- 
tensionsstellung und der minimalen Ventralneigung von der Flexions- zur Neutralstellung gegeben (Tabelle 13).

Dorsale Vorlastpositionen und die Resektionen der Zwischenwirbelgelenke vergrößerten die Ventralneigung (Tabelle 10). Wie auch bei ventraler Vorlast und im intakten Segment verkleinerte sie sich im Bewegungsablauf von der Extension zur Flexion. Die Verkippung der Schraubachsen nach kaudal war mit 8,28 in Extension größer als bei den vorherigen Resektionszuständen und Vorlastpositionen, bei $\alpha=0^{\circ}$ und Flexion mit $0^{\circ}$ bzw. $0,08^{\circ}$ jedoch nur gering (Tabelle 11 ).

Tabelle 10: Mittelwerte der ventro-dorsalen Abweichung der momentanen Schraubachse von einer Parallelen der y-Achse in Grad bei unterschiedlichen Segmentzuständen, Vorlastpositionen (VL) und Rotationsstadien. Besonders die Resektion des rechten Gelenks führte zu Veränderungen in der Ausrichtung der IHA. Standardabweichung in Klammern; $\alpha=$ Rotationswinkel; + bzw. - = Abweichung nach ventral bzw. dorsal; Flexion-Extension

\begin{tabular}{|c|c|cc|cc|cc|}
\hline Segmentzustand & VL-Position & \multicolumn{2}{|c|}{$\boldsymbol{\alpha}=\mathbf{0}^{\circ}$} & \multicolumn{2}{c|}{ max. Extension } & \multicolumn{2}{c|}{ max. Flexion } \\
\hline \multirow{4}{*}{ intaktes Segment } & zentral & 4,3 & $( \pm 4,6)$ & 3,8 & $( \pm 7,8)$ & 1,0 & $( \pm 5,4)$ \\
\cline { 2 - 9 } & ventral & $-0,1$ & $( \pm 0,6)$ & 1,4 & $( \pm 2,5)$ & $-3,1$ & $( \pm 14,4)$ \\
\cline { 2 - 9 } & dorsal & 5,4 & $( \pm 10,4)$ & 9,1 & $( \pm 12,0)$ & 1,4 & $( \pm 8,1)$ \\
\hline \multirow{4}{*}{$\begin{array}{c}\text { nach Resektion des } \\
\text { rechten Gelenks }\end{array}$} & zentral & $-10,3$ & $( \pm 9,0)$ & $-12,0$ & $( \pm 13,6$ & $-6,6$ & $( \pm 4,0)$ \\
\cline { 2 - 9 } & ventral & $-9,0$ & $( \pm 6,5)$ & $-3,4$ & $( \pm 1,7)$ & $-10,4$ & $( \pm 6,8)$ \\
\cline { 2 - 9 } & dorsal & $-3,2$ & $( \pm 24,3)$ & $-5,9$ & $( \pm 25,8)$ & 3,9 & $( \pm 19,1)$ \\
\hline \multirow{3}{*}{$\begin{array}{c}\text { Bandscheiben-Bänder- } \\
\text { Präparat }\end{array}$} & zentral & 7,0 & $( \pm 6,3)$ & 8,5 & $( \pm 10,2)$ & 5,2 & $( \pm 4,6)$ \\
\cline { 2 - 8 } & ventral & 2,3 & $( \pm 2,7)$ & 3,9 & $( \pm 2,6)$ & 6,0 & $( \pm 16,0)$ \\
\cline { 2 - 8 } & dorsal & 8,8 & $( \pm 11,6)$ & 10,9 & $( \pm 13,9$ & 6,3 & $( \pm 9,5)$ \\
\hline
\end{tabular}

Tabelle 11: Mittelwerte der kranio-kaudalen Abweichung der momentanen Schraubachse von einer Parallelen der y-Achse in Grad bei unterschiedlichen Segmentzuständen, Vorlastpositionen (VL) und Rotationsstadien. Die Abweichung der IHA von der Drehmomentachse war tendenziell gering. Standardabweichung in Klammern; $\alpha$ = Rotationswinkel; + bzw. - = Abweichung nach kranial bzw. kaudal; Flexion-Extension

\begin{tabular}{|c|c|cc|cc|cc|}
\hline Segmentzustand & VL-Position & \multicolumn{2}{|c|}{$\boldsymbol{\alpha}=\mathbf{0}^{\circ}$} & \multicolumn{2}{|c|}{ max. Extension } & \multicolumn{2}{c|}{ max. Flexion } \\
\hline \multirow{4}{*}{ intaktes Segment } & zentral & $-0,9$ & $( \pm 1,5)$ & $-1,3$ & $( \pm 4,3)$ & $-0,7$ & $( \pm 1,6)$ \\
\cline { 2 - 9 } & ventral & $-4,0$ & $( \pm 10,1)$ & $-1,3$ & $( \pm 1,9)$ & 0,6 & $( \pm 3,3)$ \\
\cline { 2 - 9 } & dorsal & $-2,9$ & $( \pm 4,2)$ & $-0,7$ & $( \pm 6,5)$ & $-3,9$ & $( \pm 3,7)$ \\
\hline \multirow{4}{*}{$\begin{array}{c}\text { nach Resektion des } \\
\text { rechten Gelenks }\end{array}$} & zentral & 3,3 & $( \pm 15,7)$ & $-6,3$ & $( \pm 18,4)$ & 2,5 & $( \pm 8,0)$ \\
\cline { 2 - 9 } & ventral & $-0,2$ & $( \pm 4,0)$ & 0,2 & $( \pm 1,7)$ & 0,1 & $( \pm 4,6)$ \\
\cline { 2 - 8 } & dorsal & $-4,5$ & $( \pm 6,9)$ & $-4,3$ & $( \pm 6,1)$ & $-5,4$ & $( \pm 8,0)$ \\
\hline \multirow{4}{*}{$\begin{array}{c}\text { Bandscheiben-Bänder- } \\
\text { Präparat }\end{array}$} & zentral & $-1,0$ & $( \pm 6,6)$ & $-1,2$ & $( \pm 10,9)$ & $-0,6$ & $( \pm 3,6)$ \\
\cline { 2 - 8 } & ventral & 0,7 & $( \pm 0,5)$ & 1,5 & $( \pm 1,0)$ & 0,6 & $( \pm 0,5)$ \\
\cline { 2 - 8 } & dorsal & 0,0 & $( \pm 12,0)$ & $-8,3$ & $( \pm 19,2)$ & $-0,1$ & $( \pm 6,8)$ \\
\hline
\end{tabular}




\subsubsection{Laterale Verschiebung der Vorlast}

Bei linksseitig positionierter Vorlast am intakten Segment war die Schraubachse in Extension nach dorsal und minimal nach kaudal verkippt. Im Laufe der Flexion verringerte sich der dorsale Neigungswinkel, der kaudale blieb konstant. In sagittaler Richtung verhielt sich die Achsbewegung somit gegensätzlich zu der bei zentraler Position. War die Vorlast rechts, lag, ähnlich wie bei zentraler Vorlast, in Extension eine Schraubachsneigung nach ventral und leicht nach kaudal vor. In Flexionsstellung kippte die Achse weiterhin nach kaudal, die Ventralneigung verringerte sich stark. Generell war die sagittale Abweichung stärker ausgeprägt als bei zentraler Vorlast.

Nach der ersten Resektion ähnelte der Verlauf der Achsbewegung demjenigen des intakten Segments, der Neigungswinkel war jedoch größer. Die Ausrichtung der Achsen in vertikaler Richtung blieb sowohl von der Vorlastverschiebung als auch von der Gelenkresektion unbeeinflusst und wich nur sehr dezent von der y-Achse nach kaudal ab. Auch rechte Vorlastpositionen führten zu Achsabweichungen wie im intakten Segment. Im Unterschied zur linken Vorlast und dem unresezierten Segment verringerte sich die Ventralneigung jedoch bei großen Extensionswinkeln wieder deutlich. Die Kaudalneigung war stärker ausgeprägt als bei linken Vorlastpositionen.

Im Vergleich zum intakten Segment verringerte sich bei den Bandscheiben-BänderPräparaten die Ausrichtung nach ventral bzw. dorsal während diejenige nach kranial bzw. kaudal dezent zunahm. Die zuvor beobachtete Verstärkung der Ventral- bzw. Dorsalkippung durch Extension war nun weniger stark ausgeprägt.

\subsubsection{Veränderung der Größe der Vorlast}

Weder einer Verringerung noch eine Erhöhung der Vorlast führte zu einer veränderten Lage der Schraubachsen der intakten Segmente.

Durch Eliminierung der Vorlast vergrößerte sich nach der Resektion des rechten Gelenks nur die Dorsalneigung leicht, auch im Vergleich zum intakten Segment. Auch eine Vorlast von $400 \mathrm{~N}$ führte zu einer Verstärkung der Dorsalneigung. Weiterhin nahm diese Neigung während der Extensionsbewegung ab. Dieser Effekt war zuvor weniger deutlich. Die geringe Verkippung nach kaudal blieb erhalten.

Am Bandscheiben-Bänder-Präparat ging eine Veränderung der Vorlastgröße nur in Extension mit einer Abweichung nach ventral und kaudal einher. Die kaudale Komponente war bei Erhöhung der Vorlast deutlicher als nach Verringerung.

\subsubsection{Drehwinkel-Drehmoment-Kennlinie und Bewegungsausmaß}

\subsubsection{Sagittale Verschiebung der Vorlast}

Die Form der Drehwinkel-Drehmoment-Kennlinie war abhängig von der Vorlastposition. So wurde die Sigmoidität bei ventralen Vorlasten von etwa 10-20 mm deutlich (Bandscheiben-Bänder-Präparat: 20-40 mm). Weiter ventral oder dorsal gelegene Vorlastpositionen führten eher zu einer „Kornähren-Form“ der Kennlinie (Abbildung 50). 


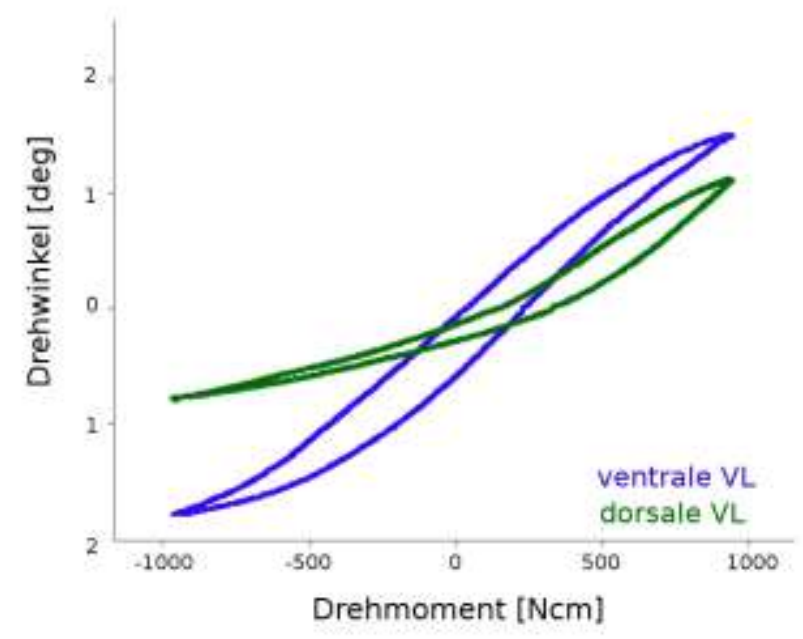

Abbildung 50: Drehwinkel-Drehmoment-Kennlinie bei ventraler und dorsaler Vorlast; Flexion-Extension. Ventrale Vorlastpositionen gingen mit einer sigmoiden, dorsale mit einer „Kornähren“-Form der Kennlinie einher.

Die Hysterese nahm bei allen Resektionszuständen durch Verschiebung der Vorlast von ventral nach dorsal stetig ab. Nach jeder Resektion war sie größer als zuvor.

\section{Bewegungsausmaß (ROM)}

Bei Betrachtung des range of motion (ROM) konnte bei jedem Segmentzustand eine deutliche Verringerung des Bewegungsausmaßes festgestellt werden je weiter dorsal die Vorlast lag (Abbildung 51 und Tabelle 12). Legte man den ROM des intakten Segments bei zentraler Vorlast $\left(2,22^{\circ}\right)$ als Referenzwert mit $100 \%$ fest, ergab sich bei $40 \mathrm{~mm}$ ventraler Vorlast ein Anstieg auf $158 \%\left(3,38^{\circ}\right)$, bei entsprechender dorsaler Position ein Absinken auf $46 \%\left(1,02^{\circ}\right)$.

Nach den Resektionen zeigte sich eine Minderung des Bewegungsausmaßes, wenn die Vorlast über $40 \mathrm{~mm}$ hinaus nach ventral verschoben wurde. Weiterhin führten besonders ventrale Vorlasten jeweils zu prozentual stärkeren Effekten. 


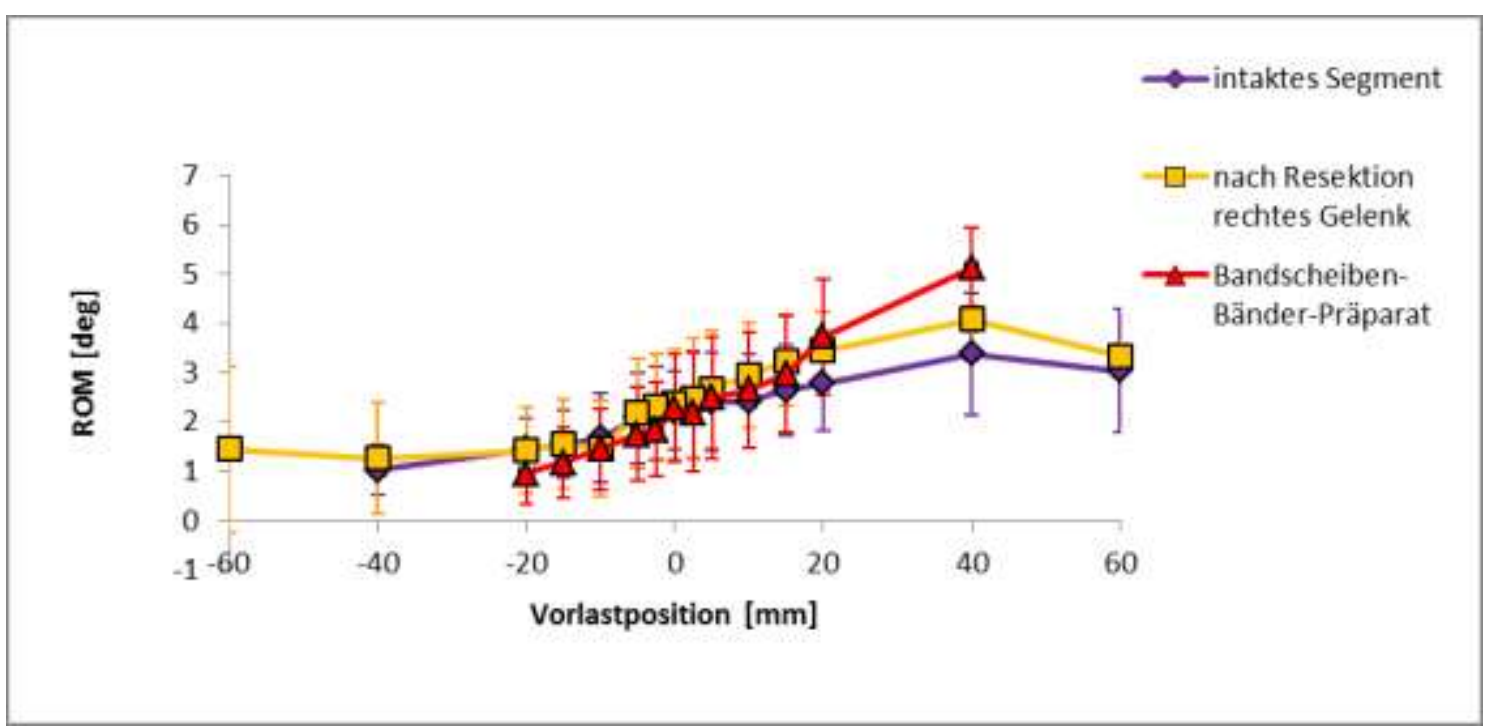

Abbildung 51: Mittlerer ROM in Grad bei allen gemessenen sagittalen Vorlastpositionen. Die Variabilität des ROM durch Verschiebung der Vorlast wurde nach jedem Resektionsschritt größer. Vorlast $200 \mathrm{~N}$; alle Resektionszustände; Flexion-Extension

Tabelle 12: Mittlerer ROM in Grad absolut und in Prozent des ROM bei zentraler Vorlast bei verschiedenen sagittalen Vorlastpositionen; alle Segmentzustände; Standardabweichung in Klammern; FlexionExtension

\begin{tabular}{|c|c|c|c|c|}
\hline $\begin{array}{l}\text { Segmentzustand/ } \\
\text { Vorlastposition (mm) }\end{array}$ & 40 dorsal & 10 dorsal & zentral & 40 ventral \\
\hline intaktes Segment & $\begin{array}{c}1,02 \quad( \pm 0,51) \\
46 \%\end{array}$ & $\begin{array}{c}1,68 \quad( \pm 0,89) \\
84 \%\end{array}$ & $\begin{array}{c}2,22 \quad( \pm 0,79) \\
100 \%\end{array}$ & $\begin{array}{c}3,38 \quad( \pm 1,23) \\
158 \%\end{array}$ \\
\hline nach Resektion des rechten Gelenks & $\begin{array}{c}1,26 \quad( \pm 1,13) \\
49 \%\end{array}$ & $\begin{array}{c}1,45 \quad( \pm 0,96) \\
74 \%\end{array}$ & $\begin{array}{c}2,36 \quad( \pm 1,11) \\
100 \%\end{array}$ & $\begin{array}{c}4,08 \quad( \pm 0,28) \\
212 \%\end{array}$ \\
\hline Bandscheiben-Bänder-Präparat & & $\begin{array}{c}1,45 \quad( \pm 0,82) \\
61 \%\end{array}$ & $\begin{array}{c}2,28 \quad( \pm 1,09) \\
100 \%\end{array}$ & $\begin{array}{c}5,13 \quad( \pm 0,8) \\
296 \%\end{array}$ \\
\hline
\end{tabular}

\subsubsection{Laterale Verschiebung der Vorlast}

Im intakten Segment und Bandscheiben-Bänder-Präparat war die Breite der Hysterese vergleichbar und blieb durch laterale Vorlasten unbeeinflusst. Nach Resektion des rechten Zwischenwirbelgelenks verbreiterte sich die Hysterese, besonders und gleichermaßen bei lateralen Vorlastpositionen.

\section{Bewegungsausmaß (ROM)}

Durch Lateralverschiebung der Vorlast um $5 \mathrm{~mm}$ erhöhte sich der range of motion der intakten Segmente, um nach weiterer Verschiebung leicht abzufallen. Auch nach der ersten Resektion war das Bewegungsausmaß, nun verstärkt, nach geringer Lateralisierung am stärksten (Abbildung 52). Auf Seiten des verbliebenen Gelenks verhielt es sich wie beim intakten Segment. Bei Verschiebung der Vorlast nach rechts nahm es stetig ab, um bei Positionen über $20 \mathrm{~mm}$ wieder zuzunehmen. Beim Bandscheiben- 
Bänder-Präparat sank der ROM im Gegensatz zu den vorherigen Messungen beidseits bis zu oben genannter Position und stieg danach dezent an.

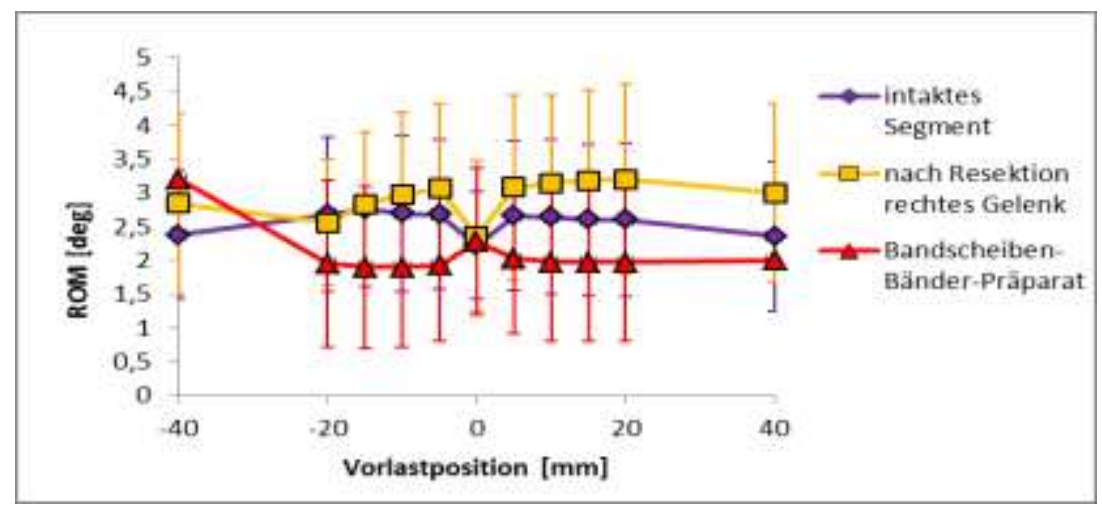

Abbildung 52: Mittlerer ROM in Grad bei allen gemessenen lateralen Vorlastpositionen. Im intakten Segment und nach Resektion des rechten Gelenks führte eine Lateralverschiebung der Vorlast zu einem Anstieg des ROM, nach Resektion beider Gelenke zu einem Absinken. Vorlast 200 N; alle Resektionszustände; Flexion-Extension

\subsubsection{Veränderung der Größe der Vorlast}

Eine Erhöhung der Größe der Vorlast führte bei allen Resektionsständen zu einer Verschmälerung der Hysterese, Messungen ohne Vorlast zur Verbreiterung. Der Kurvenverlauf war bei erhöhter Vorlast eher kornährenförmig, während eine Verringerung der Vorlast zu sigmoiden Kurven führte.

Das Bewegungsausmaß nahm bei allen Messungen durch Vergrößerung der Vorlast ab, durch Verringerung zu. Die Effekte waren nach jedem Resektionsschritt, besonders bei Elimination der Vorlast, ausgeprägter.

\subsubsection{Steifigkeitsverhalten}

Abhängig vom Rotationsausmaß veränderte sich die Steifigkeit. Minimale Werte wurden ungefähr zum Zeitpunkt der geringsten Rotation $\left(\alpha \approx 0^{\circ}\right)$ erreicht. Die höchste Steifigkeit fand sich an den Umkehrpunkten der Drehrichtung, also in maximaler Flexionsbzw. Extensionsstellung. Bei ventralen Vorlastpositionen von etwa 10-20 mm verlief die Kurve parabelförmig. War die Vorlast weiter dorsal angebracht, verkürzte sich der Schenkel bei positiven Drehwinkeln (Flexion) während derjenige auf der Extensionsseite länger wurde (Abbildung 53). Weiterhin sank die Parabelbreite mit zunehmender Dorsalisierung der Vorlast und stieg nach jedem Resektionsschritt. 


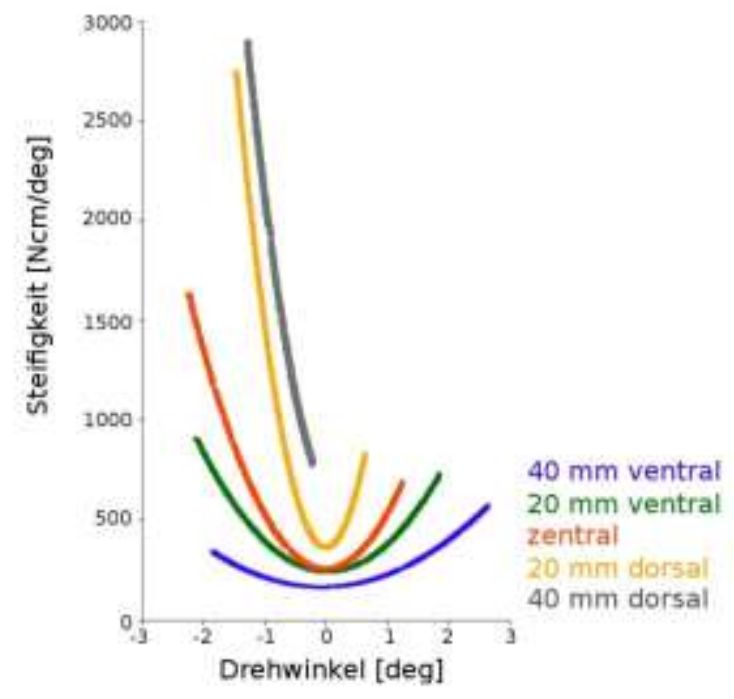

Abbildung 53: Steifigkeitskurven bei unterschiedlichen Vorlastpositionen. Die Steifigkeit stieg parallel zur Dorsalverlagerung der Vorlast $(200 \mathrm{~N})$ und mit steigender Dorsalextension. Flexion-Extension

\subsubsection{Sagittale Verschiebung der Vorlast}

Beim intakten Segment führte eine Vorlastverschiebung von zentral nach $40 \mathrm{~mm}$ dorsal zur Erhöhung der minimalen Steifigkeit von 564 Ncm/deg (100 \%) auf 1438 Ncm/deg, also auf $319 \%$ des Wertes bei zentraler Vorlast. Bei einer Vorlastposition von 2,5 mm dorsal kam es zu einem peakartigen Anstieg auf $110 \%$. Eine Verschiebung der Vorlast um $40 \mathrm{~mm}$ nach ventral resultierte in einer Abnahme auf $364 \mathrm{Ncm} / \mathrm{deg}$, was $88 \%$ des Referenzwertes entsprach (Abbildung 54, Tabelle 13).

Nach den Resektionen nahm die Steifigkeit weiterhin bei Verlagerung der Vorlast nach ventral ab und nach Dorsalverschiebung stark zu. Der peakartige Anstieg war nicht weiter erkennbar. Eine Auffälligkeit des Bandscheiben-Bänder-Präparats bestand in der prozentual gesteigerten Steifigkeitszunahme durch dorsale Vorlastverschiebung. 


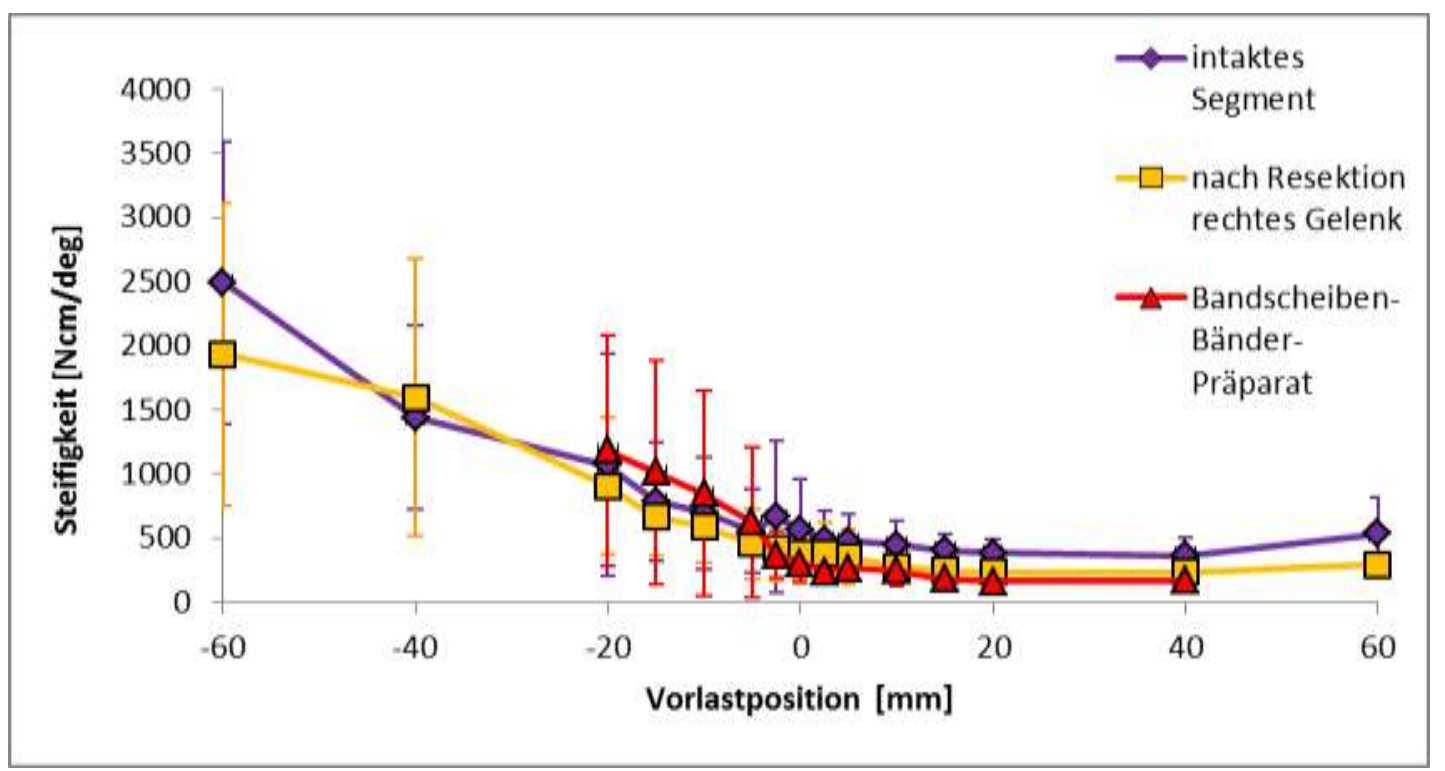

Abbildung 54: Mittlere minimale Steifigkeit in Ncm/deg bei allen gemessenen sagittalen Vorlastpositionen. Die Grafik zeigt einen peakartigen Anstieg der Steifigkeit der intakten Segmente bei gering dorsalen Vorlastpositionen. Nach den Resektionen war dieser Effekt nicht nachweisbar. Vorlast $200 \mathrm{~N}$; alle Resektionszustände; Flexion-Extension

Tabelle 13: Mittlere minimale Steifigkeit in Ncm absolut und in Prozent der Steifigkeit bei zentraler Vorlast bei verschiedenen sagittalen Vorlastpositionen; Vorlast 200 N; alle Segmentzustände; Standardabweichung in Klammern; Flexion-Extension

\begin{tabular}{|c|c|c|c|c|c|}
\hline $\begin{array}{l}\text { Segmentzustand/ } \\
\text { Vorlastposition (mm) }\end{array}$ & 40 dorsal & 10 dorsal & 5 dorsal & zentral & 40 ventral \\
\hline intaktes Segment & $\begin{array}{c}1438 \quad( \pm 720) \\
319 \%\end{array}$ & $\begin{array}{c}694 \quad( \pm 436) \\
131 \%\end{array}$ & $\begin{array}{c}552( \pm 329) \\
104 \%\end{array}$ & $\begin{array}{c}564( \pm 396) \\
100 \%\end{array}$ & $\begin{array}{c}364 \quad( \pm 141) \\
88 \%\end{array}$ \\
\hline $\begin{array}{l}\text { nach Resektion des } \\
\text { rechten Gelenks }\end{array}$ & $\begin{array}{c}1596( \pm 1084) \\
438 \%\end{array}$ & $\begin{array}{c}590 \quad( \pm 287) \\
158 \%\end{array}$ & $\begin{array}{c}452( \pm 270) \\
115 \%\end{array}$ & $\begin{array}{c}378( \pm 200) \\
100 \%\end{array}$ & $\begin{array}{c}232 \quad( \pm 59) \\
75 \%\end{array}$ \\
\hline $\begin{array}{c}\text { Bandscheiben-Bänder- } \\
\text { Präparat }\end{array}$ & & $\begin{array}{c}1013( \pm 875) \\
\mathbf{2 4 2} \%\end{array}$ & $\begin{array}{c}588( \pm 588) \\
179 \%\end{array}$ & $\begin{array}{c}305( \pm 152) \\
100 \%\end{array}$ & $\begin{array}{c}167 \quad( \pm 38) \\
68 \%\end{array}$ \\
\hline
\end{tabular}

\subsubsection{Laterale Verschiebung der Vorlast}

Beim intakten Segment und nach der ersten Resektion kam es durch laterale Positionierung der Vorlasten ( $5 \mathrm{~mm}$ ) zunächst zu einem rasanten Absinken der Steifigkeit auf etwa zwei Drittel des Ausgangswerts. Eine weitere Lateralisierung bis $40 \mathrm{~mm}$ führte zu einem stetigen Anstieg. An diesen Maximalpositionen war die Steifigkeit vergleichbar zu derjenigen bei zentraler Vorlast (Abbildung 55).

Die Veränderungen, die am Bandscheiben-Bänder-Präparat durch Verlagerung der Vorlast nach lateral hervorgerufen wurden, waren inhomogen. Im Durchschnitt führte eine geringe Vorlastverschiebung eher zu einer Steigerung der Steifigkeit. Somit wurde ein Kontrast zum unresezierten Präparat deutlich. 


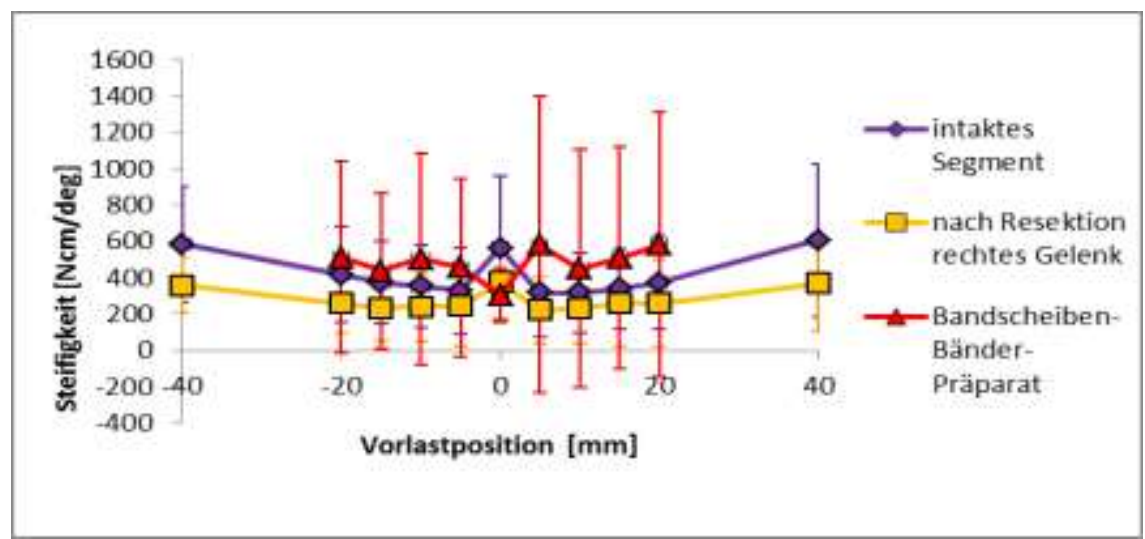

Abbildung 55: Mittlere minimale Steifigkeit in $\mathrm{Ncm} / \mathrm{deg}$ bei allen gemessenen lateralen Vorlastpositionen. Eine Lateralverschiebung der Vorlast führte beim intakten Segment und nach der Resektion des rechten Gelenks zu einem Absinken der Steifigkeit, nach Resektion beider Gelenke zu einem Anstieg. Vorlast 200 N; alle Resektionszustände; Flexion-Extension

\subsubsection{Veränderung der Größe der Vorlast}

Eine Verringerung der Vorlast führte bei allen Messungen zum Absinken der Steifigkeit und eine Erhöhung zum Anstieg. Wie auch bei den Ergebnissen zum ROM nahm das Veränderungsausmaß nach jedem Resektionsschritt zu. 


\subsubsection{Zusammenfassung der Ergebnisse: „Flexion-Extension“}

Tabelle 14: Zusammenfassung der Ergebnisse des Experiments „Flexion-Extension“

\section{Lage und Wanderung der Schraubachse}

\begin{tabular}{|c|c|c|}
\hline \multirow{3}{*}{ intaktes Segment } & $\begin{array}{c}\mathrm{VL} \\
\text { zentral }\end{array}$ & $\begin{array}{l}\text { Rastpolkurve liegt im oberen Bereich des unteren Wirbels und verläuft von } \\
\text { der Mitte nach dorso-kaudal (Extensionsbewegung) }\end{array}$ \\
\hline & $\begin{array}{c}\mathrm{VL} \\
\text { ventral }\end{array}$ & $\begin{array}{l}\text { Rastpolkurve verläuft mittig am kaudalen Bandscheibenrand nach dorsal } \\
\text { (Extensionsbewegung) }\end{array}$ \\
\hline & $\begin{array}{c}\mathrm{VL} \\
\text { dorsal }\end{array}$ & $\begin{array}{l}\text { Rastpolkurve liegt im dorsalen und kranialen Drittel des unteren Wirbels } \\
\text { und verläuft nach dorso-kaudal (Extensionsbewegung) }\end{array}$ \\
\hline \multirow{3}{*}{$\begin{array}{c}\text { nach Resektion } \\
\text { des rechten } \\
\text { Gelenks }\end{array}$} & $\begin{array}{c}\mathrm{VL} \\
\text { zentral }\end{array}$ & $\begin{array}{l}\text { Rastpolkurve ist im Vergleich zum intakten Segment dezent nach dorso- } \\
\text { kaudal versetzt }\end{array}$ \\
\hline & $\begin{array}{c}\mathrm{VL} \\
\text { ventral }\end{array}$ & $\begin{array}{l}\text { Rastpolkurve ist im Vergleich zum intakten Segment an ähnlicher Position } \\
\text { aber ortsfest }\end{array}$ \\
\hline & $\begin{array}{c}\mathrm{VL} \\
\text { dorsal }\end{array}$ & $\begin{array}{l}\text { Rastpolkurve ist im Vergleich zum intakten Segment nach dorsal versetzt } \\
\text { und ortsfest }\end{array}$ \\
\hline \multirow{3}{*}{$\begin{array}{l}\text { Bandscheiben- } \\
\text { Bänder-Präparat }\end{array}$} & $\begin{array}{c}\mathrm{VL} \\
\text { zentral }\end{array}$ & $\begin{array}{l}\text { Rastpolkurve ist ähnlich wie bei intaktem Segment, aber mit kürzerem } \\
\text { Verlauf und nach kranial versetzt }\end{array}$ \\
\hline & $\begin{array}{c}\mathrm{VL} \\
\text { ventral }\end{array}$ & $\begin{array}{l}\text { Rastpolkurve ist ähnlich wie bei intaktem Segment, aber mit kürzerem } \\
\text { Verlauf und nach kranial versetzt }\end{array}$ \\
\hline & $\begin{array}{c}\text { VL } \\
\text { dorsal }\end{array}$ & $\begin{array}{l}\text { Rastpolkurve ist ähnlich wie bei intaktem Segment, aber mit kürzerem } \\
\text { Verlauf und nach kranial und ventral versetzt }\end{array}$ \\
\hline
\end{tabular}

\section{Ausrichtung der Schraubachse}

\begin{tabular}{|c|c|l|}
\hline intaktes Segment & Schraubachse verläuft ungefähr parallel zur y-Achse \\
\hline \multirow{2}{*}{$\begin{array}{c}\text { nach Resektion } \\
\text { des rechten Ge- } \\
\text { lenks }\end{array}$} & $\begin{array}{c}\text { VLntral } \\
\text { ventral }\end{array}$ & $\begin{array}{l}\text { Schraubachsen verlaufen (besonders in Flexionsstellung) von links-kaudal } \\
\text { nach rechts-kranial }\end{array}$ \\
\cline { 2 - 3 } & $\begin{array}{c}\text { VL } \\
\text { dorsal }\end{array}$ & $\begin{array}{l}\text { Schraubachsen verlaufen in Flexionsstellung von links-kranial nach rechts- } \\
\text { kaudal }\end{array}$ \\
\hline $\begin{array}{c}\text { Bandscheiben- } \\
\text { Bänder-Präparat }\end{array}$ & & Schraubachse verläuft ungefähr parallel zur y-Achse \\
\hline
\end{tabular}

\section{Steifigkeit}

Unabhängig vom Resektionszustand steigt die Steifigkeit bei Verschiebung der Vorlast nach dorsal und sinkt bei Verschiebung nach ventral. Nach den einzelnen Resektionsschritten verstärkt sich dieser Effekt. 


\subsection{Lateralflexion}

\subsubsection{Rastpolkurven}

Zur Darstellung der Rastpolkurven bei Anlegen eines veränderlichen Drehmoments parallel zur der x-Achse (anterior-posteriore Richtung) eignen sich koronare Schnittbilder durch die Wirbelkörper mit Projektionen der Zwischenwirbelgelenke. Die Aufsicht erfolgt immer von ventral.

\subsubsection{Sagittale Verschiebung der Vorlast}

\section{Intaktes Segment}

Mit zentral liegender Vorlast verliefen die Rastpolkurven bei mittleren Rotationswinkeln annähernd horizontal etwas kranial der Oberkante des LWK 4. Am Beispiel des Präparats $A$ begann sie in linksgeneigter Stellung des LWK 3 etwa $1 \mathrm{~cm}$ links der Mittellinie und endete nach gleichsinniger in Rechtsneigung ca. $9 \mathrm{~mm}$ rechts davon. Die Wanderung erfolgte mit gleichmäßiger Geschwindigkeit.

Durch ventrale Vorlastpositionen verkleinerte sich der Wanderungsradius auf die Hälfte des vorherigen Ergebnisses. Allerdings war die Wanderungsrichtung nun umgekehrt, demnach wanderte die Achse bei Rechtsneigung des Wirbels von rechts nach links und vice versa.

Bei dorsaler Vorlast zeigte das Präparat A als leichte Auffälligkeit, dass die Kurve zu den Rotationsmaxima hin um wenige Millimeter nach kaudal wanderte (Abbildung 56). 

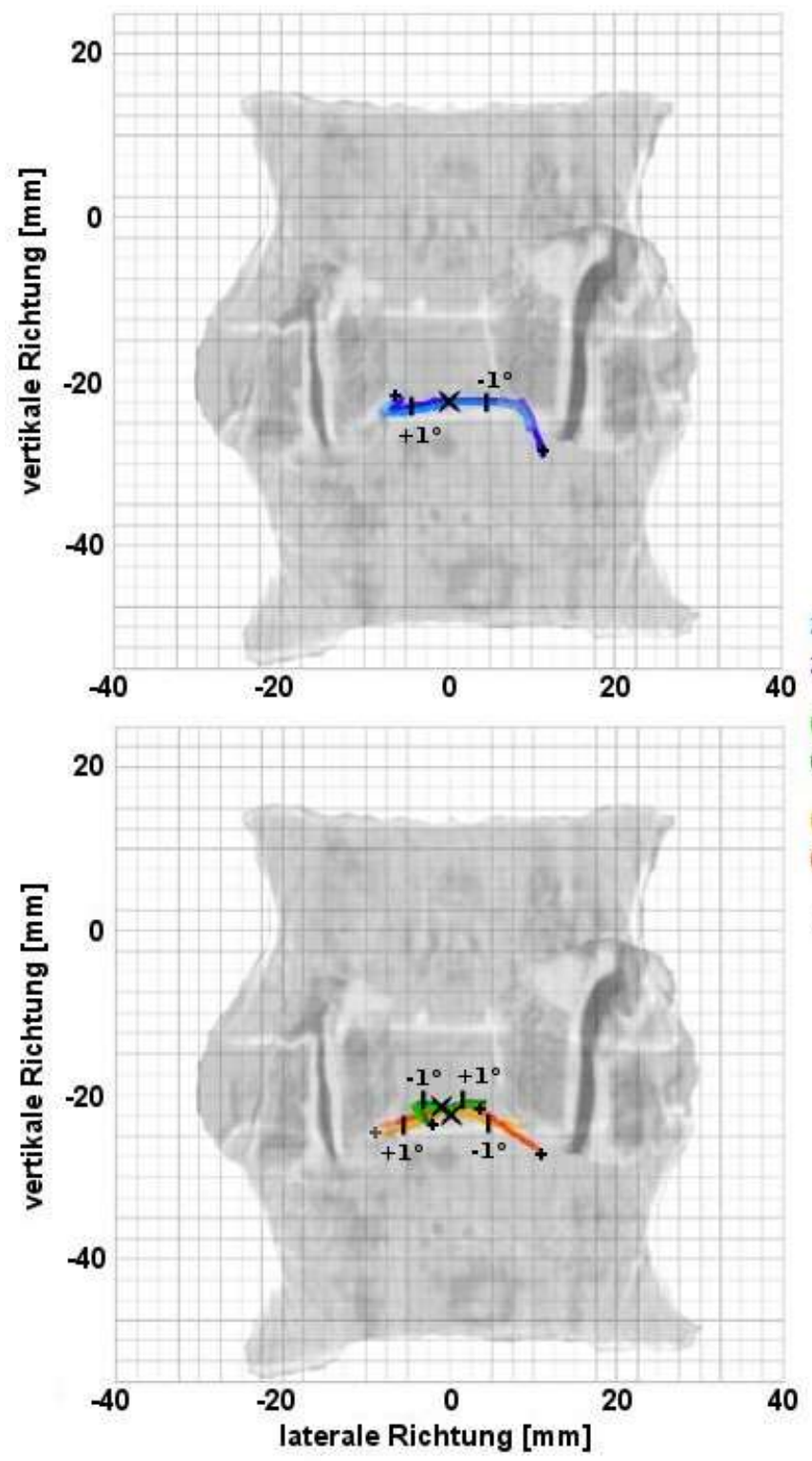

zentrale VL; Linksneigung zentrale VL; Rechtsneigung $60 \mathrm{~mm}$ ventrale $\mathrm{VL}$; Linksneigung $60 \mathrm{~mm}$ ventrale $\mathrm{VL}$; Rechtsneigung $60 \mathrm{~mm}$ dorsale VL; Linksneigung $60 \mathrm{~mm}$ dorsale $\mathrm{VL}$; Rechtsneigung $x=0^{\circ}$

Abbildung 56: Rastpolkurven des intakten Präparats A bei unterschiedlichen sagittalen Vorlastpositionen. Die Kurven liegen zwischen den beiden Gelenken und der Kurvenverlauf wurde durch ventrale Positionierung der Vorlast kürzer. Die Wanderungsrichtung verhielt sich bei ventraler Vorlastposition entgegengesetzt zu der bei zentraler und dorsaler Vorlast. Vorlast $200 \mathrm{~N}$; Lateralflexion; die Lage der Wirbelbogengelenke ist eingeblendet. + markiert den Bewegungsbeginn; die Markierungen mit Zahlen beschreiben das Rotationsausmaß.

\section{$\underline{\text { Segment nach Resektion des rechten Zwischenwirbelgelenks }}$}

Bei zentraler Vorlast verliefen die Kurven wie auch vor der Resektion etwa horizontal, jedoch knapp unterhalb der Deckplatte des unteren Wirbels (Abbildung 57 und 59). Sie nahm mit $28 \mathrm{~mm}$ nun einen weitläufigeren Verlauf und war leicht zum verbliebenen Gelenk hin versetzt.

Wurde die Vorlast ventral positioniert, lag die Rastpolkurve im Gegensatz zum intakten Segment nahezu ortsfest etwa 4 mm links der Mittellinie.

Anders als im intakten Segment resultierte auch die Verschiebung der Vorlast nach dorsal in einer geringeren Wanderung der Schraubachsen (Abbildung 57 und 60). Nun 
bewegten sie sich um $4 \mathrm{~mm}$ nach rechts und $8 \mathrm{~mm}$ nach links (Präparat A). In der Linksneigung war die Wanderung pro Zeiteinheit geringer und die Kurve lief außerdem um etwa $3 \mathrm{~mm}$ nach kranial.
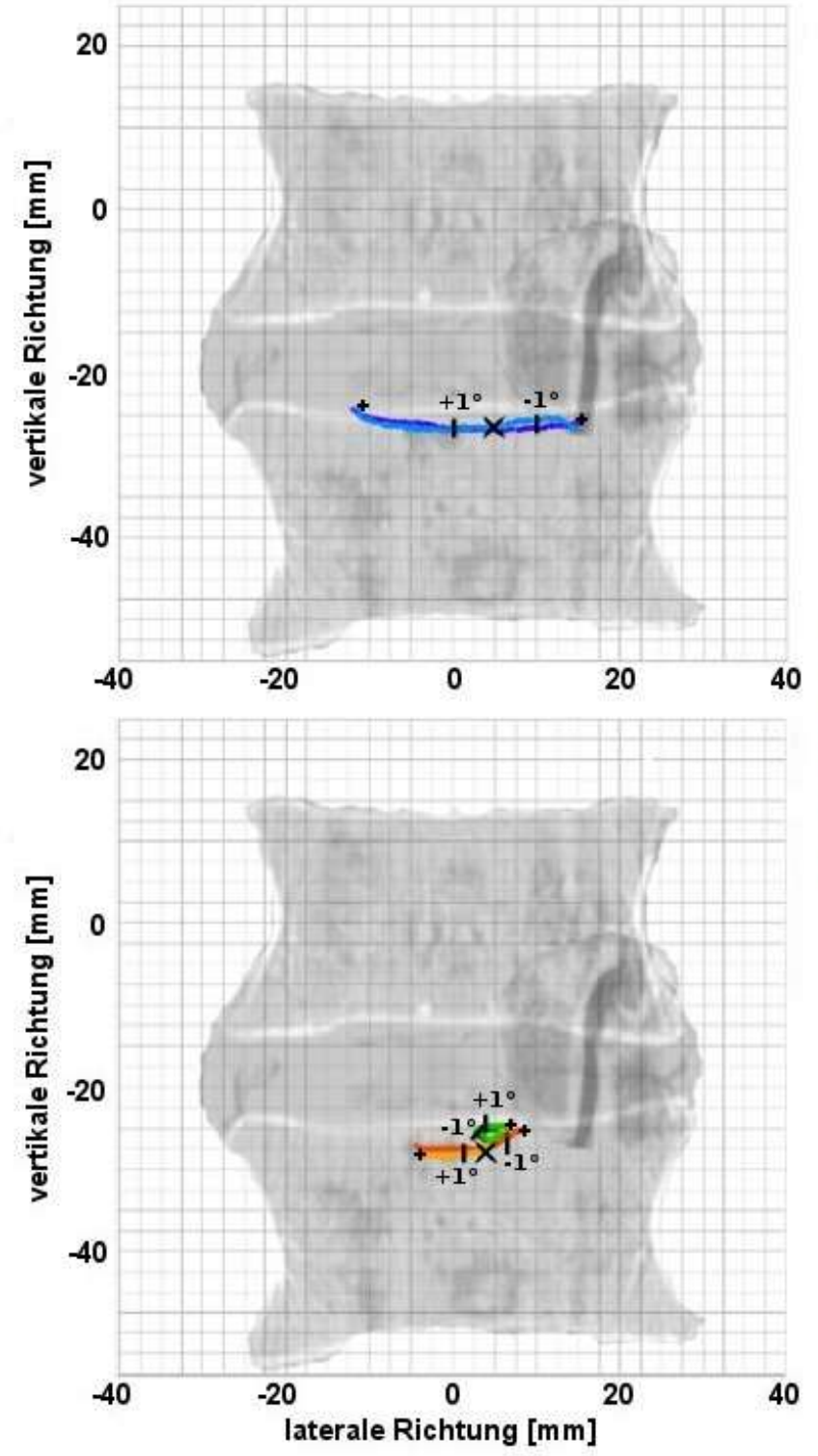

zentrale VL; Linksneigung zentrale VL; Rechtsneigung

$60 \mathrm{~mm}$ ventrale VL; Linksneigung $60 \mathrm{~mm}$ ventrale VL; Rechtsneigung

$60 \mathrm{~mm}$ dorsale VL; Linksneigung $60 \mathrm{~mm}$ dorsale VL; Rechtsneigung $x=0^{\circ}$

Abbildung 57: Rastpolkurven des Präparats A nach Resektion des rechten Wirbelbogengelenks bei unterschiedlichen sagittalen Vorlastpositionen. Die Kurven waren dezent nach links versetzt, der Kurvenverlauf bei ventraler und dorsaler Vorlastposition verkürzt. Vorlast $200 \mathrm{~N}$; Lateralflexion; die Lage des linken Wirbelbogengelenks ist eingeblendet. + markiert den Bewegungsbeginn; die Markierungen mit Zahlen beschreiben das Rotationsausmaß.

\section{Bandscheiben-Bänder-Präparat}

Im Vergleich zum intakten Segment waren die Lage und der Verlauf der Kurven nun inhomogen (Abbildung 58 und 59).

Die Verschiebung der Vorlast nach ventral führte wie auch nach der ersten Resektion zu ortsfesten Rastpolkurven, nun allerdings in der Mittellinie (Abbildung 58). 
Bei dorsaler Lage der Vorlast war die Wanderung der Schraubachsen verringert, die Position $1 \mathrm{~mm}$ weiter kaudal als bei zentraler Vorlast (Abbildung 60).

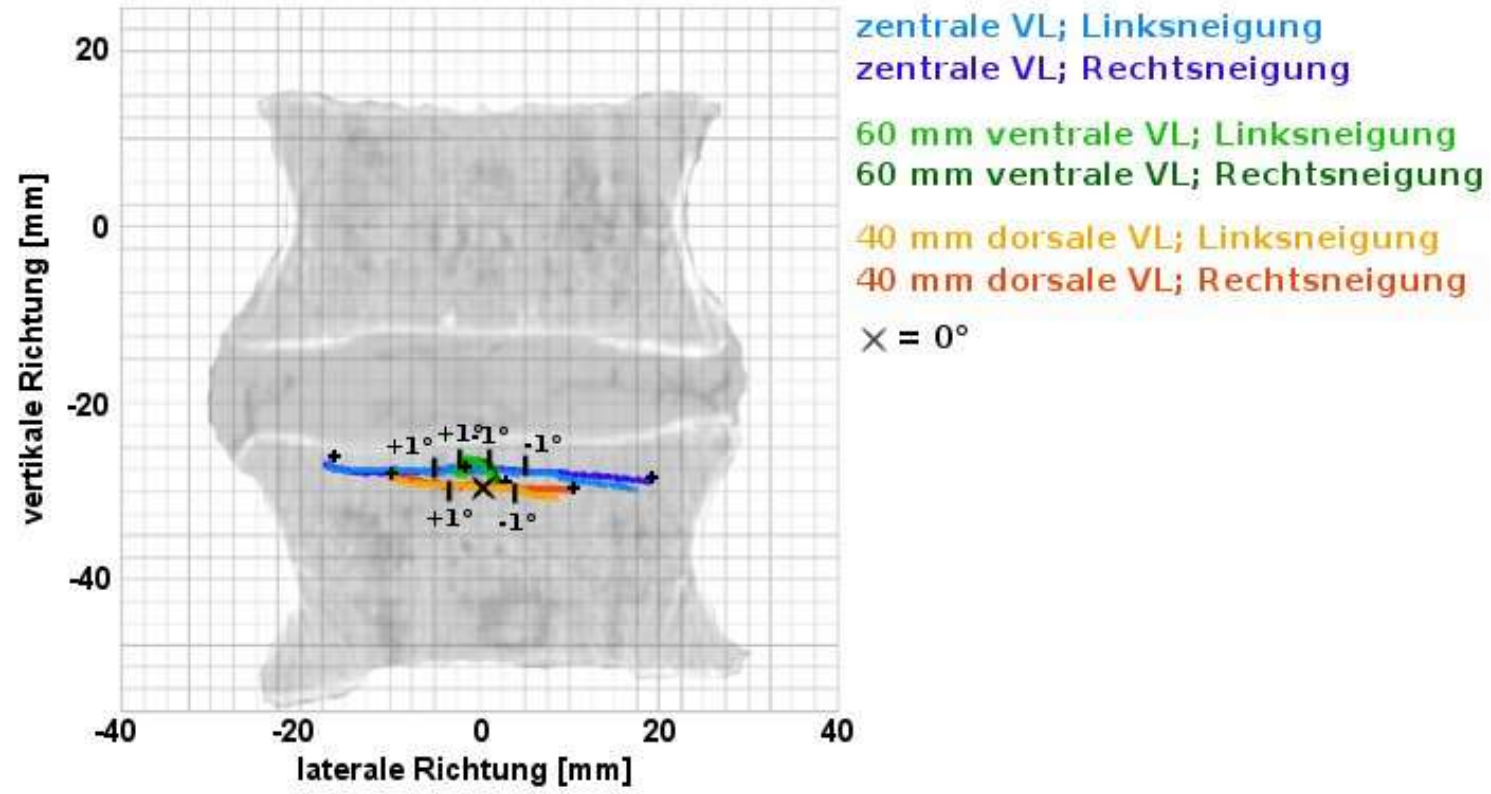

Abbildung 58: Rastpolkurven des Bandscheiben-Bänder-Präparats A bei unterschiedlichen sagittalen Vorlastpositionen. Die Vorlastposition veränderte die Länge der Kurven. Die Wanderungsrichtung war nun einheitlich (entsprechend zentraler und dorsaler Vorlast bei intaktem Segment). Vorlast 200 N; Lateralflexion; + markiert den Bewegungsbeginn; die Markierungen mit Zahlen beschreiben das Rotationsausmaß.

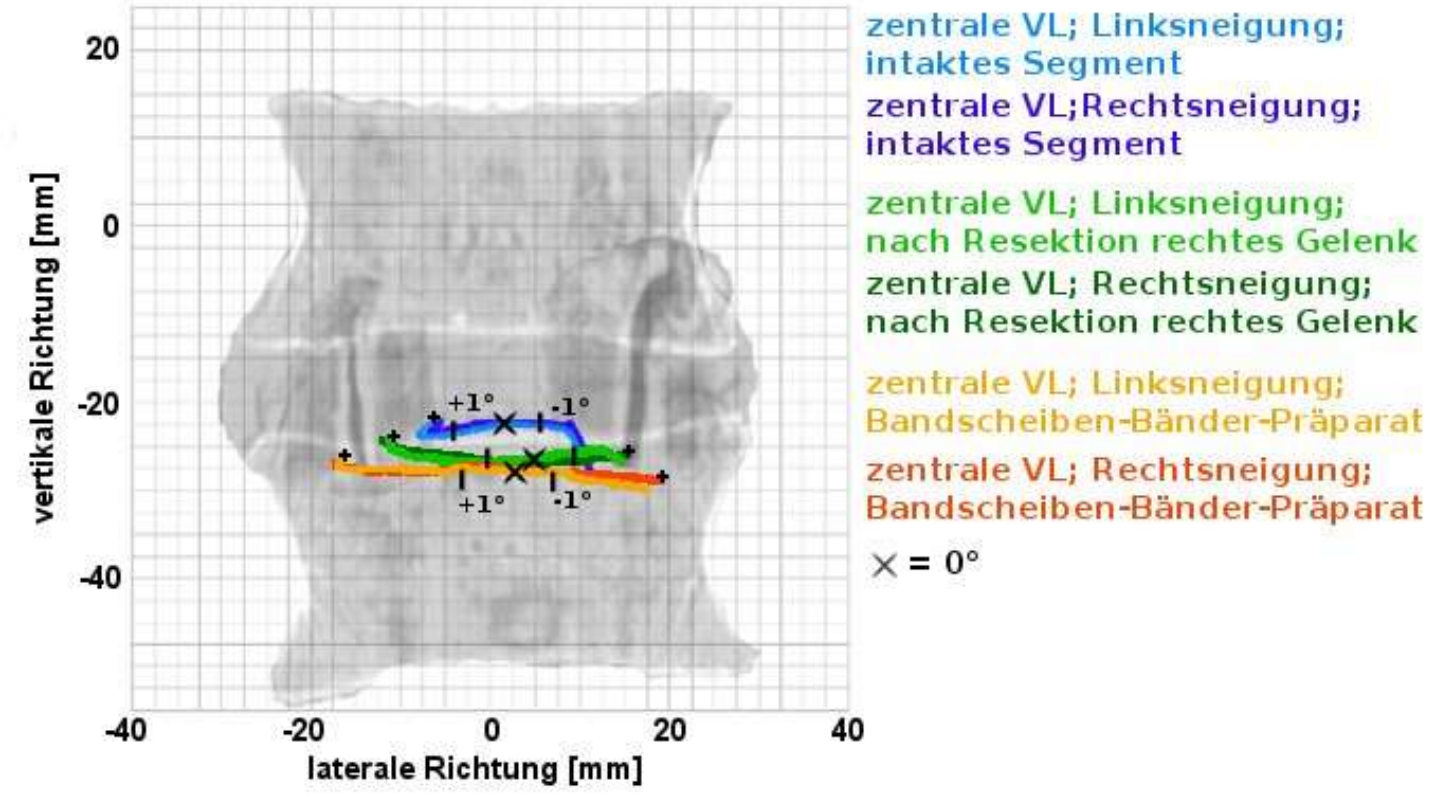

Abbildung 59: Vergleich der Rastpolkurven bei zentraler Vorlast (200 N) bei unterschiedlichen Resektionszuständen. Der Kurvenverlauf wurde nach jedem Resektionsschritt länger und war nach kaudal versetzt. Lateralflexion; + markiert den Bewegungsbeginn; die Markierungen mit Zahlen beschreiben das Rotationsausmaß. 


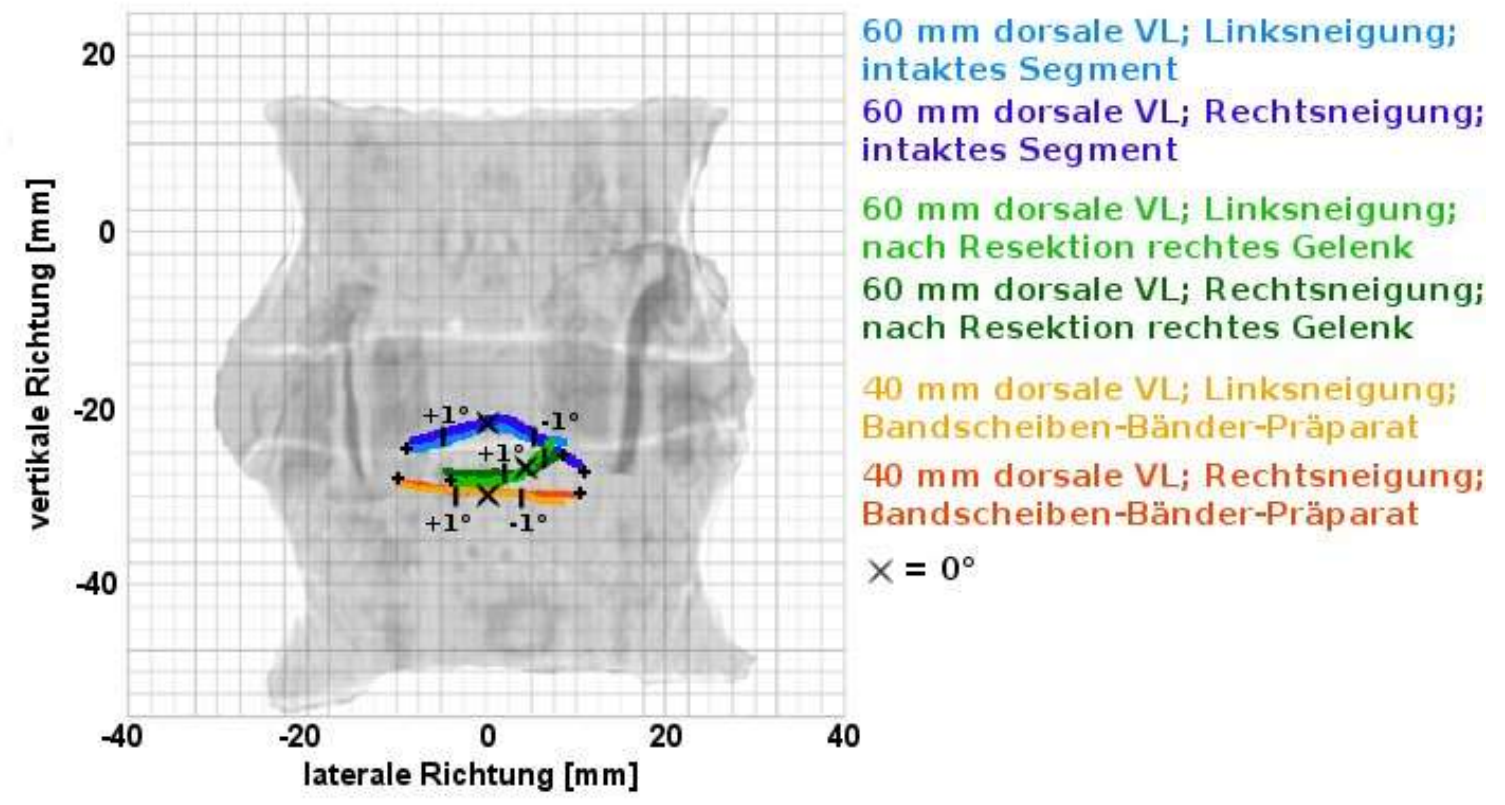

Abbildung 60: Vergleich der Rastpolkurven bei dorsaler Vorlast (200 N) bei unterschiedlichen Resektionszuständen. Nur nach einseitiger Resektion des Gelenks nahm die Kurve einen nach kranial gebogenen Verlauf in Richtung des verbliebenen Gelenks. Lateralflexion; + markiert den Bewegungsbeginn; die Markierungen mit Zahlen beschreiben das Rotationsausmaß.

\subsubsection{Laterale Verschiebung der Vorlast}

Die Verschiebung der Vorlasten nach links bei intaktem Segment bedingte eine Verlagerung der Rastpolkurven nach links sowie nach kaudal, knapp unterhalb der Deckplatte von LWK 4 (Abbildung 61).

Im Gegensatz dazu führte die rechtsseitige Vorlast zwar entsprechend zu einer Wanderung nach rechts, es fand jedoch in Abhängigkeit vom jeweiligen Präparat in den Rotationsmaxima auch eine Wanderung nach kranial oder kaudal statt. 


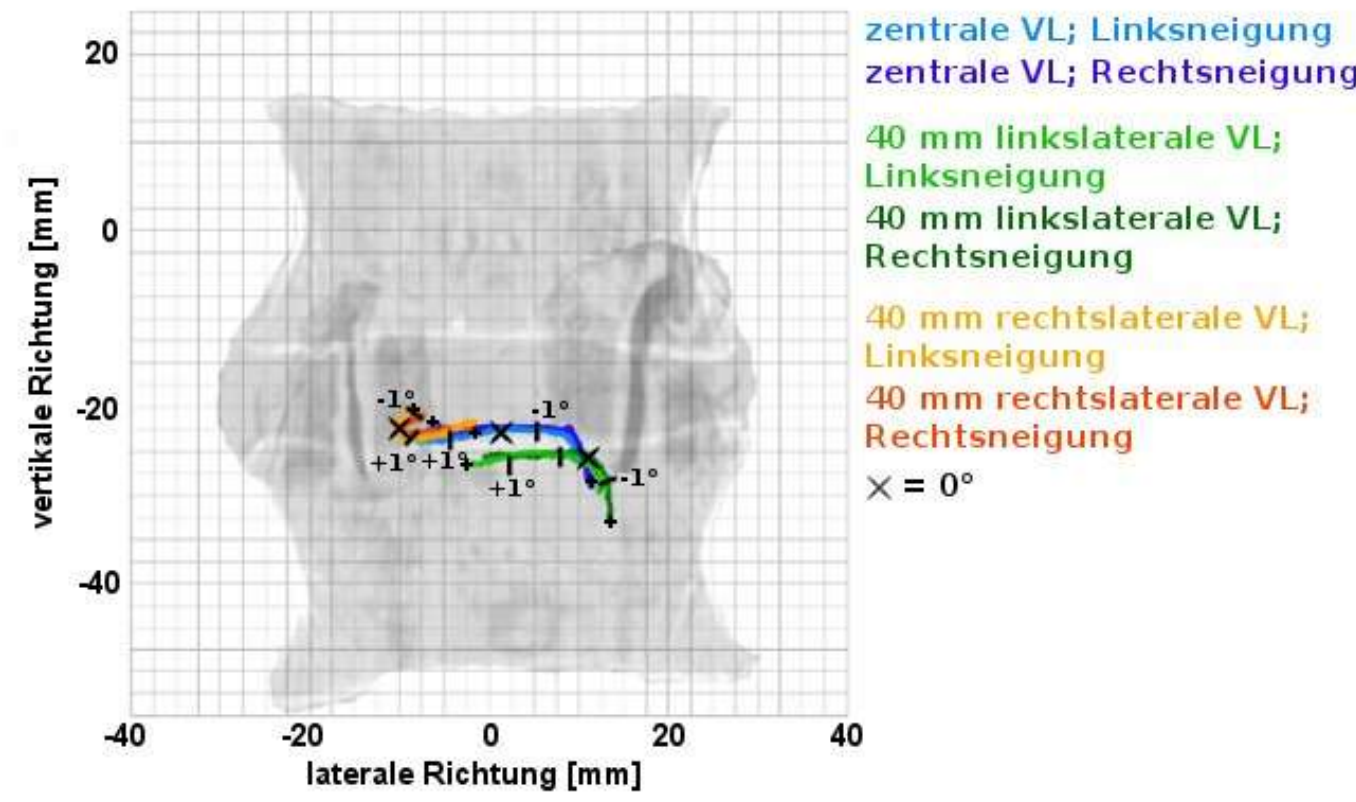

Abbildung 61: Rastpolkurven des intakten Präparats A bei unterschiedlichen lateralen Vorlastpositionen. Eine Verschiebung der Vorlast nach lateral führte zu einer Verlagerung der Kurve in Richtung der Vorlast. Vorlast $200 \mathrm{~N}$; Lateralflexion; die Lage der Wirbelbogengelenke ist eingeblendet. + markiert den Bewegungsbeginn; die Markierungen mit Zahlen beschreiben das Rotationsausmaß.

Nach der Resektion des rechten Gelenks verhielt sich die Veränderungstendenz analog zum intakten Zustand. Leichte Unterschiede konnten jedoch bei rechten Vorlasten beobachtet werden. So zeigte sich während des gesamten Rotationsverlaufs eine Verlagerung der Schraubachsen um 2 mm nach kranial (Abbildung 62).

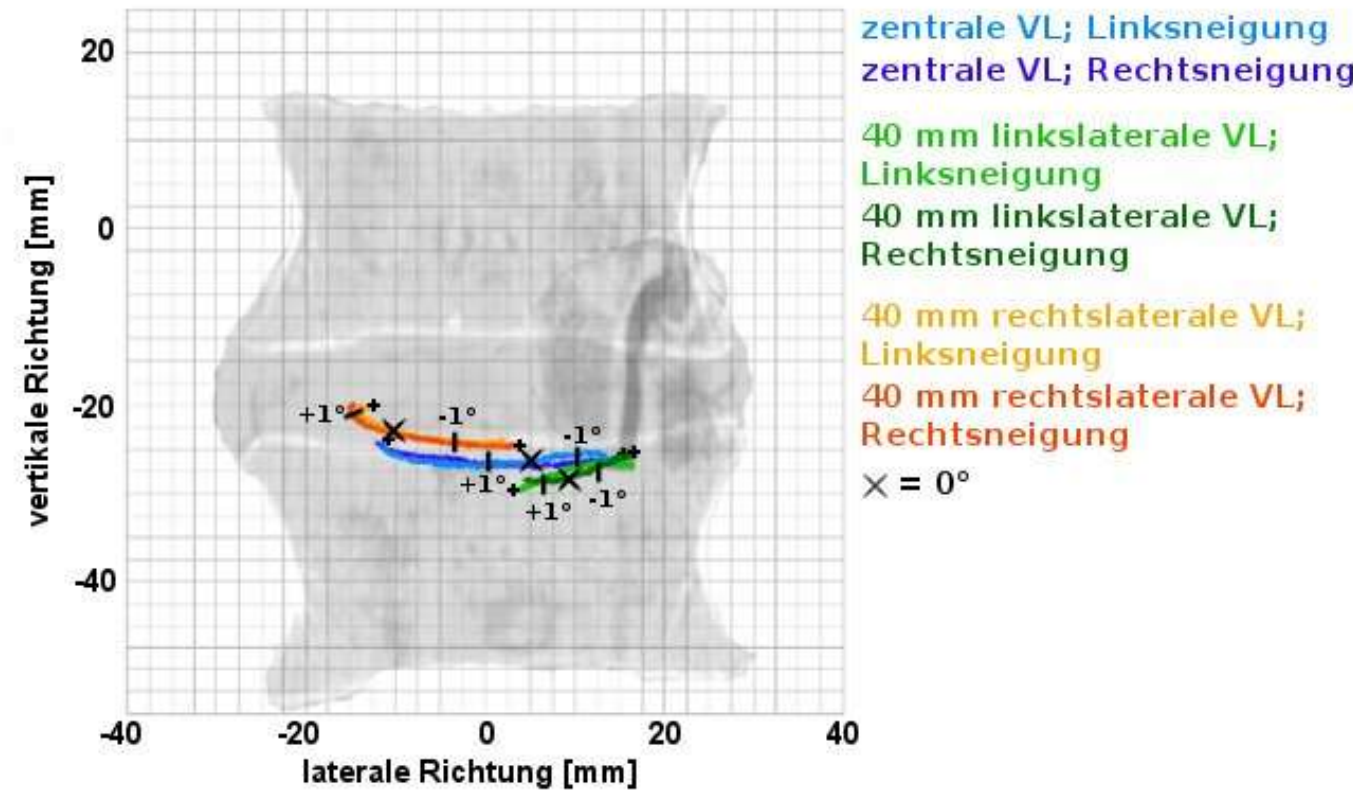

Abbildung 62: Rastpolkurven des Präparats A nach Resektion des rechten Wirbelbogengelenks bei unterschiedlichen lateralen Vorlastpositionen. Vorlast $200 \mathrm{~N}$; Lateralflexion; die Lage des Wirbelbogengelenks ist eingeblendet. + markiert den Bewegungsbeginn; die Markierungen mit Zahlen beschreiben das Rotationsausmaß. 
Am Bandscheiben-Bänder-Präparat fanden sich ähnliche Ergebnisse wie am intakten Segment.

\subsubsection{Veränderung der Größe der Vorlast}

Eine Verringerung der Vorlast auf $0 \mathrm{~N}$ ging bei intaktem Segment mit einem ortsfesteren Verlauf der Rastpolkurve einher. Durch Erhöhung auf $400 \mathrm{~N}$ kam es bei rechtsgeneigtem Segment zu einer geringgradig verlängerten Wanderung der IHA. Im Übrigen verlief die Rastpolkurve parallel zu derjenigen bei 200 N Vorlast (Abbildung 63).
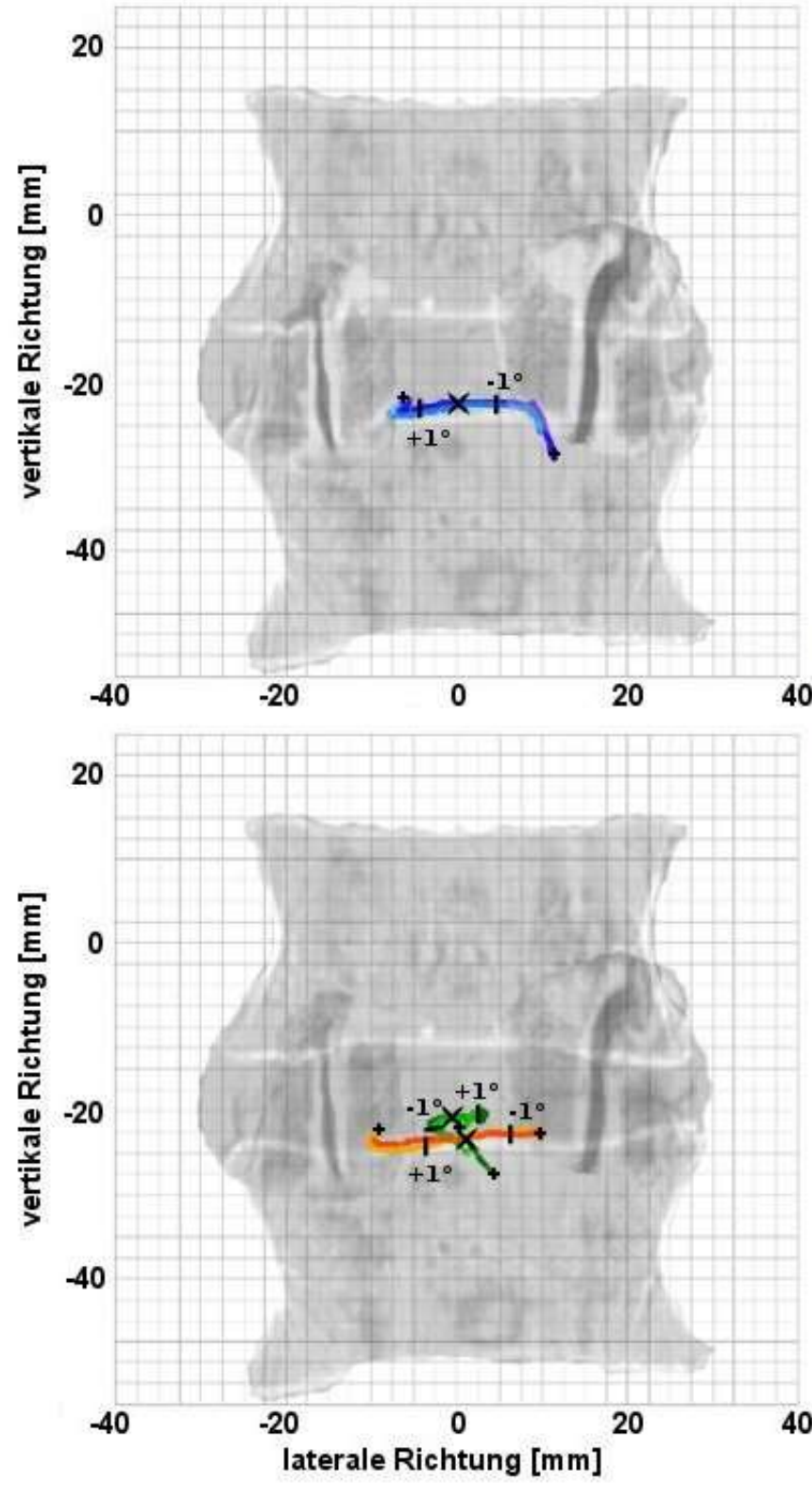

200 N VL; Linksneigung 200 N VL; Rechtsneigung 0 N VL; Linksneigung 0 N VL; Rechtsneigung

400 N VL; Linksneigung 400 N VL; Rechtsneigung $x=0^{\circ}$

Abbildung 63: Rastpolkurven des intakten Präparats A bei unterschiedlichen Vorlastgrößen. Nach Verringerung der Vorlast war die Kurve ortsfester. Lateralflexion; die Lage der Wirbelbogengelenke ist eingeblendet. + markiert den Bewegungsbeginn; die Markierungen mit Zahlen beschreiben das Rotationsausmaß. 
Nach den einzelnen Resektionsschritten gab es neben einer Verschiebung der Rastpolkurve um 2-3 mm nach kranial $(0 \mathrm{~N})$ bzw. nach kaudal $(400 \mathrm{~N})$ mit jeweils verlängertem Kurvenverlauf keine einheitlichen Effekte (Abbildung 64).

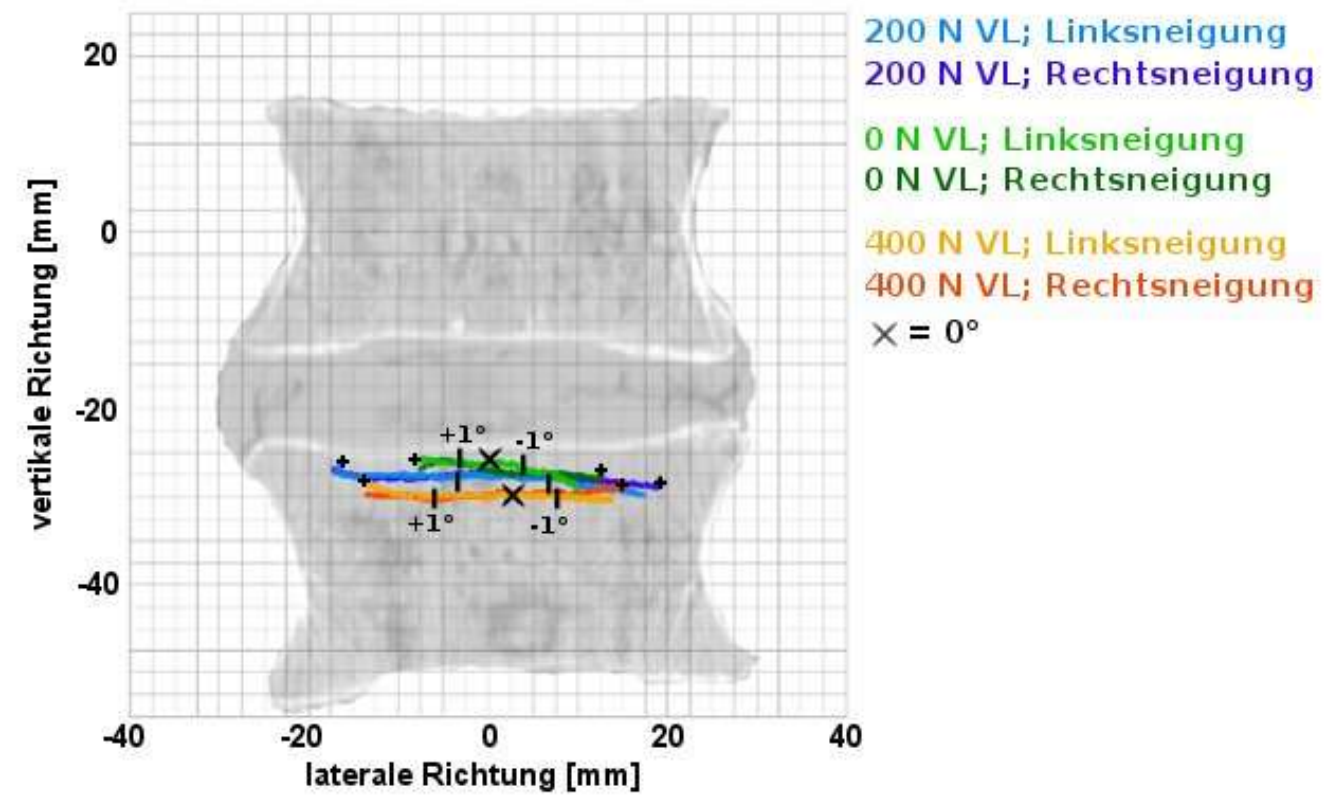

Abbildung 64: Rastpolkurven des Bandscheiben-Bänder-Präparats A bei unterschiedlichen Vorlastgrößen. Es kam zu einer geringen Verlagerung der Kurven nach kranial $(0 \mathrm{~N})$ bzw. kaudal $(400 \mathrm{~N})$; Lateralflexion; + markiert den Bewegungsbeginn; die Markierungen mit Zahlen beschreiben das Rotationsausmaß.

\subsubsection{Ausrichtung der Schraubachsen}

Im folgenden Abschnitt werden die Abweichungen der jeweiligen Schraubachsen von einer Parallelen der x-Achse dargestellt. Wie auch bei der Darstellung der Achsausrichtung des Experiments „Ventralflexion-Dorsalextension“ beziehen sich die Angaben über die Richtung der Schraubachsen (kranial-kaudal und links-rechts) auf den positiven Bereich des kartesischen Koordinatensystems. Entsprechend bedeutet eine Abweichung nach kranial einen Verlauf von ventral-kranial nach dorsal-kaudal, eine Abweichung nach rechts einen Verlauf von ventral-rechts nach dorsal-links.

\subsubsection{Sagittale Verschiebung der Vorlast}

\section{Intaktes Segment}

Bei zentralen Vorlasten und in Neutralstellung, also bei $\alpha=0^{\circ}$, verliefen die Schraubachsen annähernd parallel zur x-Achse (Tabelle 15). Die kranio-kaudalen Abweichungswinkel blieben gering mit $0,56^{\circ}$ nach kranial (Rechtsneigung) und $0,94^{\circ}$ nach kaudal (Linksneigung). Während der Seitwärtsneigung fand eine deutliche Verkippung nach lateral statt (Tabelle 16). Sie erreichte in maximaler Linksneigung durchschnittlich $13,4^{\circ}$ nach rechts, nach Rechtsneigung $12,28^{\circ}$ nach links.

Verschob man die Vorlast nach ventral, verblieb die Schraubachse in Neutralstellung entsprechend den vorangegangenen Versuchen. Im Rotationsverlauf war wiederum die kranio-kaudale Ausrichtung mit $0,85^{\circ}$ bzw. 1,15 Abweichung nach kranial annä- 
hernd konstant (Tabelle 15). Die Verkippung in laterale Richtung verlief nun jedoch entgegengesetzt: bei Linksneigung des oberen Wirbels um $14,4^{\circ}$ nach links, bei Rechtsneigung um $15,7^{\circ}$ nach rechts (Tabelle 16).

Durch dorsale Vorlastpositionen ergaben sich eher geringere Abweichungen von der $x$-Achse. Bei $\alpha=0^{\circ}$ war die Schraubachse dezent nach kranial gekippt $\left(1,83^{\circ}\right)$. Im Verlauf der Linksneigung nahm diese Verkippung leicht auf $2,33^{\circ} \mathrm{zu}$, in Gegenrichtung auf $0,01^{\circ}$ ab (Tabelle 15). Weiterhin fand sich eine Rechtsabweichung von $9,4^{\circ}$, die im Verlauf der Neigung nach rechts auf $9,88^{\circ}$ linksseitig schwenkte (Tabelle 16).

\section{$\underline{\text { Segment nach Resektion des rechten Zwischenwirbelgelenks }}$}

Bei zentral positionierter Vorlast glich die Lage der momentanen Schraubachsen in Neutralstellung $\left(\alpha=0^{\circ}\right)$ dem unresezierten Zustand. Die vertikale Ausrichtung war insgesamt inhomogen. Die Rechtsneigung der Achsen jedoch vergrößerte sich von 13,49 auf $17,85^{\circ}$ (Tabelle 16). In maximaler Rechtsneigung fiel dementsprechend eine Verringerung der Achsabweichung nach links von $12,28^{\circ}$ auf $4,71^{\circ}$ auf.

Wurde die Vorlast nach ventral verschoben, veränderte sich die Achslage in Neutralstellung kaum. Die Abweichung bei maximaler Linksneigung war von Präparat zu Präparat verschieden. In Rechtsneigung verkippten die Schraubachsen nun nach rechts $\left(16,53^{\circ}\right)$, die kranio-kaudale Neigung blieb inhomogen (Tabelle 15). Im Vergleich zum intakten Segment fiel die verringerte und innerhalb der verschiedenen Präparate ungleichere Achslage in lateraler Ausrichtung bei maximaler Linksneigung auf.

Bei dorsalen Vorlasten erschien die Lage der Achsen auch in Neutralstellung je nach Präparat variabel, stärker als bei den vorherigen Vorlastpositionen. Die Abweichungen waren eher gering. In Linksneigung erfolgte eine im Vergleich zur zentralen Vorlastposition geringere Achswanderung nach rechts $\left(9,32^{\circ}\right)$. Im Vergleich zum intakten Segment war diese jedoch in den meisten Präparaten deutlicher ausgeprägt (Tabelle 16). Auch in Rechtsneigung war die Abweichung der Schraubachsen mit 1,94 nach links und $1,49^{\circ}$ nach kaudal dezent. Diese geringe Linkswanderung zeigt den größten Unterschied zum unresezierten Segment.

\section{Bandscheiben-Bänder-Präparat}

Bei zentraler Vorlastposition war die Schraubachslage in etwa vergleichbar mit der des intakten Segments. Lediglich in Rechtsflexion schien die Linksabweichung leicht verringert

Wurde die Vorlast nach ventral verlagert, verliefen die Schraubachsen während der gesamten Bewegung von ventral-kranial nach dorsal-kaudal. Maximal waren sie um $2,7^{\circ}$ geneigt (Tabelle 15). Außerdem zeigte sich bei $\alpha=0^{\circ}$ die im Vergleich zum unresezierten Zustand deutlich geringere Abweichung um 5,03 ${ }^{\circ}$ nach links in Linksneigung und um $1,45^{\circ}$ nach rechts in Rechtsneigung (Tabelle 16).

Im Gegensatz dazu glichen die Lagetendenzen mit dorsalen Vorlastpositionen wieder denen bei zentraler Vorlast. Allerdings war die laterale Abweichung mit $3,52^{\circ} \mathrm{bzw} .3^{\circ}$ deutlich geringer. 
Tabelle 15: Mittelwerte der kranio-kaudalen Abweichung der momentanen Schraubachse von einer Parallelen der x-Achse in Grad bei unterschiedlichen Segmentzuständen, Vorlastpositionen (VL) und Rotationsstadien. Die Schraubachsen lagen bei $\alpha=0^{\circ}$ parallel zur x-Achse. Die Neigung durch das Drehmoment führte unabhängig vom Resektionszustand bei ventraler Vorlast im Vergleich zu zentraler und dorsaler Vorlast zu einer entgegengesetzten Achsausrichtung. Standardabweichung in Klammern; $\alpha=$ Rotationswinkel; + bzw. - = Abweichung nach kranial bzw. kaudal; Lateralflexion

\begin{tabular}{|c|c|cc|cc|cc|}
\hline \multirow{2}{*}{ Segmentzustand } & VL-Position & \multicolumn{2}{|c|}{$\boldsymbol{\alpha}=\mathbf{0}^{\circ}$} & \multicolumn{2}{c|}{$\begin{array}{c}\text { max. Linksnei- } \\
\text { gung }\end{array}$} & $\begin{array}{c}\text { max. Rechtsnei- } \\
\text { gung }\end{array}$ \\
\hline \multirow{4}{*}{ intaktes Segment } & zentral & 0,9 & $( \pm 0,6)$ & 0,6 & $( \pm 2,9)$ & $-0,9$ & $( \pm 2,8)$ \\
\cline { 2 - 9 } & ventral & $-0,3$ & $( \pm 0,3)$ & 0,9 & $( \pm 2,5)$ & 1,2 & $( \pm 4,0)$ \\
\cline { 2 - 9 } & dorsal & 1,8 & $( \pm 1,6)$ & 2,3 & $( \pm 4,4)$ & 0,0 & $( \pm 2,6)$ \\
\hline \multirow{4}{*}{$\begin{array}{c}\text { nach Resektion des } \\
\text { rechten Gelenks }\end{array}$} & zentral & 0,9 & $( \pm 1,4)$ & 1,1 & $( \pm 1,8)$ & $-1,1$ & $( \pm 4,2)$ \\
\cline { 2 - 9 } & ventral & $-0,6$ & $( \pm 0,8)$ & 0,3 & $( \pm 2,2)$ & $-0,3$ & $( \pm 4,4)$ \\
\cline { 2 - 9 } & dorsal & 1,0 & $( \pm 2,0)$ & 2,7 & $( \pm 3,1)$ & $-1,5$ & $( \pm 2,8)$ \\
\hline \multirow{4}{*}{$\begin{array}{c}\text { Bandscheiben-Bänder- } \\
\text { Präparat }\end{array}$} & zentral & 0,1 & $( \pm 1,8)$ & 0,8 & $( \pm 2,3)$ & $-1,6$ & $( \pm 3,6)$ \\
\cline { 2 - 9 } & ventral & 0,6 & $( \pm 1,6)$ & 2,7 & $( \pm 2,8)$ & 1,3 & $( \pm 2,2)$ \\
\cline { 2 - 8 } & dorsal & 0,7 & $( \pm 2,0)$ & 1,1 & $( \pm 3,8)$ & $-0,9$ & $( \pm 2,4)$ \\
\hline
\end{tabular}

Tabelle 16: Mittelwerte der lateralen Abweichung der momentanen Schraubachse von einer Parallelen der x-Achse in Grad bei unterschiedlichen Segmentzuständen, Vorlastpositionen (VL) und Rotationsstadien. Die Abweichung von der $x$-Achse war stets gering. Standardabweichung in Klammern; $\alpha=$ Rotationswinkel; + bzw. - = Abweichung nach links bzw. rechts; Lateralflexion

\begin{tabular}{|c|c|c|c|c|c|c|c|}
\hline Segmentzustand & VL-Position & \multicolumn{2}{|c|}{$\alpha=0^{\circ}$} & \multicolumn{2}{|c|}{$\begin{array}{l}\text { max. Linksnei- } \\
\text { gung }\end{array}$} & \multicolumn{2}{|c|}{$\begin{array}{c}\text { max. Rechtsnei- } \\
\text { gung }\end{array}$} \\
\hline \multirow{3}{*}{ intaktes Segment } & zentral & 0,0 & $( \pm 0,0)$ & $-13,9$ & $( \pm 4,4)$ & 12,3 & $( \pm 4,7)$ \\
\hline & ventral & 0,0 & $( \pm 0,0)$ & 14,8 & $( \pm 3,0)$ & $-15,8$ & $( \pm 6,2)$ \\
\hline & dorsal & 0,3 & $( \pm 0,0)$ & $-9,4$ & $( \pm 5,9)$ & 9,9 & $( \pm 2,5)$ \\
\hline \multirow{3}{*}{$\begin{array}{l}\text { nach Resektion des } \\
\text { rechten Gelenks }\end{array}$} & zentral & $-1,2$ & $( \pm 2,9)$ & $-17,9$ & $( \pm 2,9)$ & 4,7 & $( \pm 4,6)$ \\
\hline & ventral & $-2,3$ & $( \pm 5,8)$ & 1,3 & $( \pm 6,2)$ & $-16,5$ & $( \pm 6,5)$ \\
\hline & dorsal & 0,0 & $( \pm 4,2)$ & $-9,3$ & $( \pm 7,1)$ & 1,9 & $( \pm 4,3)$ \\
\hline \multirow{3}{*}{$\begin{array}{c}\text { Bandscheiben-Bänder- } \\
\text { Präparat }\end{array}$} & zentral & 0,1 & $( \pm 0,3)$ & $-14,0$ & $( \pm 11,8)$ & 9,5 & $( \pm 9,6)$ \\
\hline & ventral & 1,9 & $( \pm 1,5)$ & 5,0 & $( \pm 4,7)$ & $-1,5$ & $( \pm 8,0)$ \\
\hline & dorsal & 1,0 & $( \pm 0,7)$ & $-3,5$ & $( \pm 9,8)$ & 3,0 & $( \pm 5,4)$ \\
\hline
\end{tabular}

\subsubsection{Laterale Vorlastposition}

Eine linksseitig positionierte Vorlast beim intakten Segment führte im Wesentlichen zu einer Veränderung der Schraubachslage in der horizontalen Ebene. So war die Achse bei maximaler Linksneigung nach rechts verdreht. Im Verlauf der Rechtsneigung verringerte sich die Abweichung von der Parallelen zur x-Achse ab der Neutralstellung, um in maximaler Neigung gegen null zu gehen. 
Lag die Vorlast auf der rechten Seite zeigte sich analog das umgekehrte Bild: In der nach links geneigten Stellung des Wirbels glich die Schraubachse annähernd der Ausrichtung des Drehmoments, bei Rechtsneigung fand eine Abweichung nach links statt.

Nach den Resektionen war jeweils die Achsabweichung insgesamt, nach der ersten Resektion insbesondere die Rechtskippung, deutlicher als zuvor.

\subsubsection{Veränderung der Größe der Vorlast}

Die Veränderung der Vorlast bedingte inhomogene, nur geringfügige Effekte an der Schraubachsausrichtung.

\subsubsection{Drehwinkel-Drehmoment-Kennlinie und Bewegungsausmaß}

\subsubsection{Sagittale Verschiebung der Vorlast}

Die Drehwinkel-Drehmoment-Kennlinie hatte wie auch bei den axialen Versuchen die typische sigmoide Form, jedoch geringer ausgeprägt. Weiterhin nahm diese Sigmoidität mit zunehmend dorsalen Vorlastpositionen ab, so dass die Kurve bei $60 \mathrm{~mm}$ dorsaler Vorlast beinahe linear verlief.

Durch Verlagerung der Vorlast von ventral über zentral nach dorsal verschmälerte sich die Hysterese. Nach jedem Resektionsschritt war sie breiter als zuvor.

\section{Bewegungsausmaß (ROM): intaktes Segment}

Wie auch bei den anderen Rotationsrichtungen war das Bewegungsausmaß bei ventralen Vorlastpositionen größer als bei dorsalen. Die Durchschnittswerte betrugen $8,02^{\circ}(110 \%)$ bei $60 \mathrm{~mm}$ ventraler, $7,22^{\circ}$ (100\%) bei zentraler und 5,84 ${ }^{\circ}$ (81\%) bei dorsal liegender Vorlast. Ein geringer Anstieg des ROM $\left(7,6^{\circ}\right)$ konnte bei $10 \mathrm{~mm}$ dorsaler Vorlast festgestellt werden (Abbildung 65, Tabelle 17).

\section{Segment nach Resektion des rechten Zwischenwirbelgelenks}

Das Bewegungsausmaß nahm, wie auch im intakten Segment, ab, je weiter dorsal die Vorlast angebracht war (Abbildung 65). Insgesamt war es leicht erhöht, ausgeprägter bei dorsalen Vorlastpositionen $\left(6,78^{\circ}\right)$. Der ROM stieg bei geringen dorsalen Vorlastpositionen $\left(10 \mathrm{~mm}\right.$ ) von $7,52^{\circ}$ auf $8,38^{\circ}$ an und war somit in dieser Position etwas ausgeprägter als vor der Resektion. Das Maximum betrug $8,32^{\circ}$ bei $60 \mathrm{~mm}$ ventraler Vorlast. Die prozentualen Veränderungen durch Ventralisierung der Vorlast lagen um $108 \%$, bei dorsalen Vorlasten um $83 \%$ (Tabelle 17). 


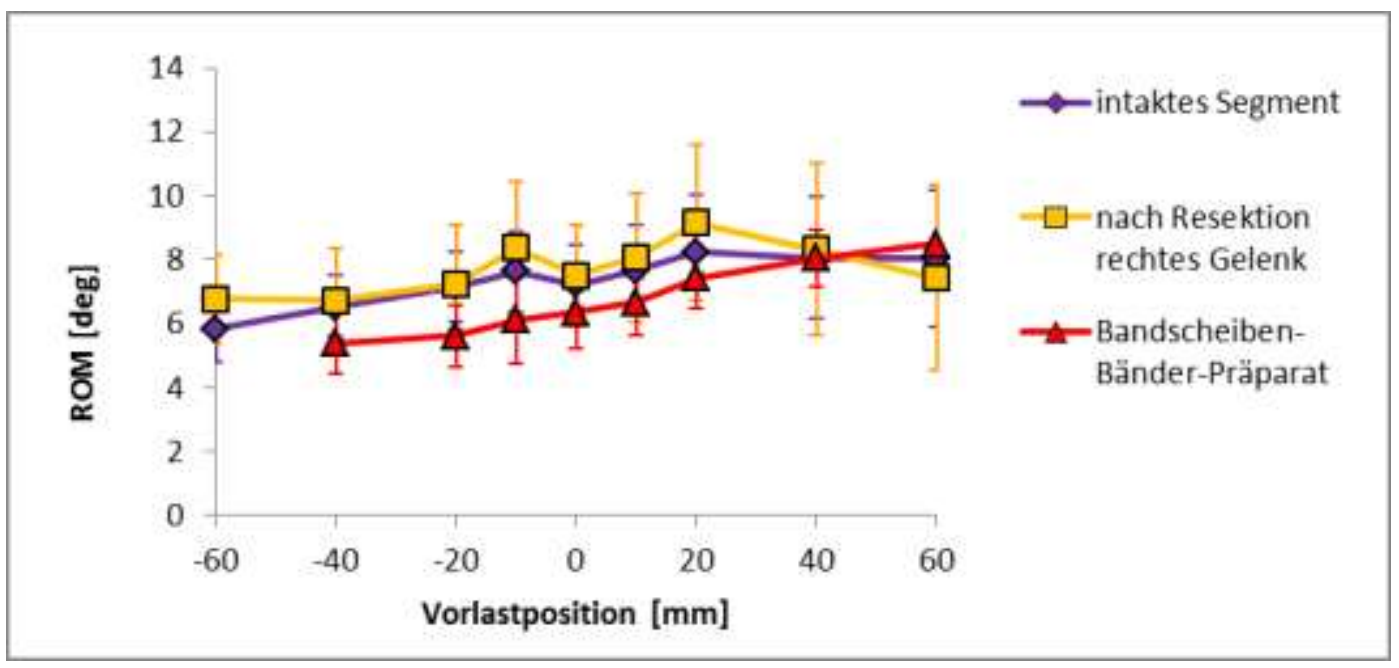

Abbildung 65: Mittlerer ROM in Grad bei allen gemessenen sagittalen Vorlastpositionen. Die Kurven der intakten und einseitig resezierten Segmente zeigten eine Zunahme des ROM bei gering dorsalen Vorlasten, das Bandscheiben-Bänder-Präparat nicht. Vorlast 200 N; alle Resektionszustände; Lateralflexion

\section{Bandscheiben-Bänder-Präparat}

Der range of motion nahm durch die Vorlastverschiebung von zentral nach ventral zu, nach dorsal hingegen stetig ab. Der zuvor sichtbare Peak bei leicht dorsalen Positionen konnte nicht dargestellt werden. Die Durchschnittswerte erreichten $8,05^{\circ}$ bei $40 \mathrm{~mm}$ ventraler, $6,35^{\circ}$ in zentraler und $5,4^{\circ}$ bei dorsaler Vorlastlage. Prozentual ergaben sich mit 129 \% bzw. 86 \% ausgeprägtere Effekte als vor den Resektionen, insgesamt war das Bewegungsausmaß allerdings geringer (Abbildung 65, Tabelle 17).

Tabelle 17: Mittlerer ROM in Grad absolut und in Prozent des ROM bei zentraler Vorlast bei verschiedenen Vorlastpositionen. Die prozentualen Veränderungen der Steifigkeit durch Vorlastverschiebung waren nach beidseitiger Resektion dezent verstärkt. Vorlast 200 N; alle Segmentzustände; Standardabweichung in Klammern; Lateralflexion

\begin{tabular}{|c|c|c|c|c|c|}
\hline $\begin{array}{l}\text { Segmentzustand/ } \\
\text { Vorlastposition (mm) }\end{array}$ & $\begin{array}{c}60 \\
\text { dorsal }\end{array}$ & $\begin{array}{c}40 \\
\text { dorsal }\end{array}$ & zentral & $\begin{array}{c}40 \\
\text { ventral }\end{array}$ & $\begin{array}{c}60 \\
\text { ventral }\end{array}$ \\
\hline intaktes Segment & $\begin{array}{c}5,84( \pm 1,03) \\
81 \%\end{array}$ & $\begin{array}{c}6,52( \pm 1,02) \\
91 \%\end{array}$ & $\begin{array}{c}7,22( \pm 1,24) \\
100 \%\end{array}$ & $\begin{array}{c}8,06( \pm 1,89) \\
111 \%\end{array}$ & $\begin{array}{c}8,02( \pm 2,14) \\
110 \%\end{array}$ \\
\hline $\begin{array}{c}\text { nach Resektion des } \\
\text { rechten Gelenks }\end{array}$ & $\begin{array}{c}6,78( \pm 1,38) \\
83 \%\end{array}$ & $\begin{array}{c}6,72( \pm 1,65) \\
89 \%\end{array}$ & $\begin{array}{c}7,52( \pm 1,59) \\
100 \%\end{array}$ & $\begin{array}{c}8,32( \pm 2,7) \\
108 \%\end{array}$ & $\begin{array}{c}7,43( \pm 2,9) \\
82 \%\end{array}$ \\
\hline $\begin{array}{l}\text { Bandscheiben- } \\
\text { Bänder-Präparat }\end{array}$ & & $\begin{array}{c}5,4( \pm 0,96) \\
86 \%\end{array}$ & $\begin{array}{c}6,35( \pm 1,14) \\
100 \%\end{array}$ & $\begin{array}{c}8,05( \pm 0,9) \\
129 \%\end{array}$ & \\
\hline
\end{tabular}




\subsubsection{Laterale Verschiebung der Vorlast}

\section{Breite der Hysterese}

Die Veränderungen waren zu jedem Messzeitpunkt inhomogen.

Bewegungsausmaß (ROM):

Beim intakten Segment sank das Bewegungsausmaß durch Lateralverschiebung der Vorlast, besonders innerhalb der ersten $10 \mathrm{~mm}$. Auch nach der Resektion des rechten Gelenks fand sich dieser Effekt. Allerdings fiel auf, dass bei rechtsseitiger Vorlast ein stärker Abfall stattfand. Beim Bandscheiben-Bänder-Präparat zeigten sich ähnliche Verhältnisse bei insgesamt durch die Reduktion des Drehmoments bedingt niedrigerem range of motion (Abbildung 66).

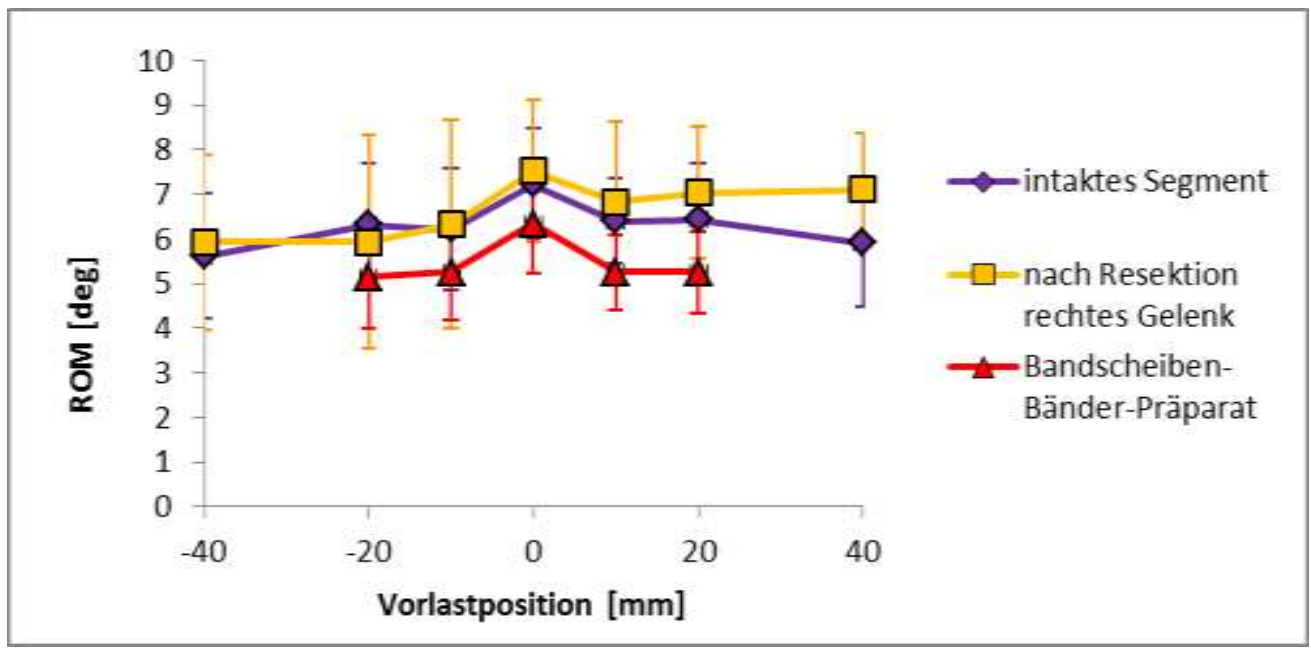

Abbildung 66: Mittlerer ROM in Grad bei allen gemessenen lateralen Vorlastpositionen. Das Bewegungsausmaß nahm unabhängig vom Resektionszustand durch Lateralverschiebung der Vorlast ab. Vorlast 200 N; alle Resektionszustände; Lateralflexion

\subsubsection{Veränderung der Größe der Vorlast}

Homogene Effekte fanden sich lediglich am Bandscheiben-Bänder-Präparat. Dort nahm die Breite der Hysterese durch Verminderung der Vorlast zu, durch Erhöhung ab.

Bewegungsausmaß (ROM)

Der range of motion verkleinerte sich stets mit wachsender Vorlast. Mit jedem Resektionsschritt verstärkten sich diese Effekte.

\subsubsection{Steifigkeitsverhalten}

\subsubsection{Sagittale Verschiebung der Vorlast}

Die Steifigkeit der intakten Segmente lag bei zentraler Vorlast von $200 \mathrm{~N}$ bei $214 \mathrm{Ncm} /$ deg. Nach Ventralverschiebung der Vorlast sank sie innerhalb der ersten $10 \mathrm{~mm}$ deutlich, danach nur noch gering bis $168 \mathrm{Ncm} / \mathrm{deg}$ ab (Abbildung 67). Während der Dorsalisierung blieb sie zunächst annähernd konstant, um in $60 \mathrm{~mm}$ dorsaler Posi- 
tion das Maximum mit $334 \mathrm{Ncm} / \mathrm{deg} z u$ erreichen. Prozentual variierte die Steifigkeit in Abhängigkeit von der Vorlastposition zwischen $76 \%$ (ventrale Vorlast) und $156 \%$ (dorsale) Vorlast, wenn der Wert bei zentraler Vorlast als Referenz angesehen wurde (Tabelle 18).

Nach der Resektion des rechten Gelenks verhielt sich die Steifigkeit der Präparate bei Verlagerung der Vorlast von zentral nach dorsal inhomogen. Im Mittel resultierte eine Vergrößerung von $184 \mathrm{Ncm} / \mathrm{deg}$ auf $262 \mathrm{Ncm} / \mathrm{deg}$. Der Effekt des Steifigkeitsanstiegs durch Ventralisierung der Vorlast von $40 \mathrm{~mm}$ auf $60 \mathrm{~mm}$ stand im Gegensatz zum intakten Segment (Abbildung 67). Das Minimum der Steifigkeit lag bei $40 \mathrm{~mm}$ ventral liegender Vorlast bei etwa $140 \mathrm{Ncm} / \mathrm{deg}$. Prozentual war die Steifigkeitszunahme, wie auch die Steifigkeit insgesamt, durch dorsale Vorlasten mit $126 \%$ des zentralen Wertes geringer als vor der Resektion (Tabelle 18).

Beim Bandscheiben-Bänder-Präparat verlief der Anstieg durch Dorsalverschiebung der Vorlast gleichmäßiger. Zwischen $10 \mathrm{~mm}$ ventral und $40 \mathrm{~mm}$ dorsal war die Steifigkeit höher als bei den vorherigen Resektionsständen. So wurden Werte von $235 \mathrm{Ncm} / \mathrm{deg}$ bei zentraler, $128 \mathrm{Ncm} /$ deg bei ventraler und $303 \mathrm{Ncm} /$ deg bei dorsaler Vorlast erreicht (Abbildung 67).

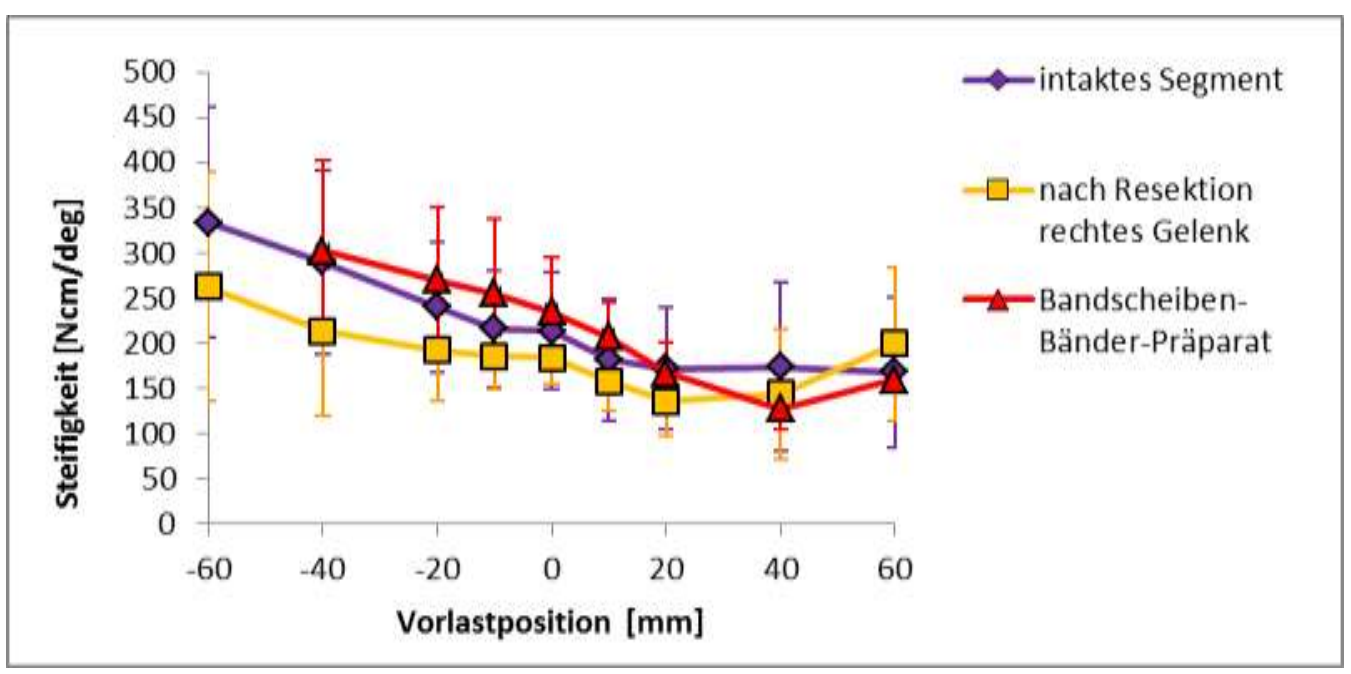

Abbildung 67: Mittlere minimale Steifigkeit in $\mathrm{Ncm} / \mathrm{deg}$ bei allen gemessenen sagittalen Vorlastpositionen. Vorlast $200 \mathrm{~N}$; alle Resektionszustände; Lateralflexion 
Tabelle 18: Mittlere minimale Steifigkeit in Ncm absolut und in Prozent der Steifigkeit bei zentraler Vorlast bei verschiedenen sagittalen Vorlastpositionen. Veränderungen der Steifigkeit durch Verschiebung der Vorlast waren am intakten Segment besonders durch Dorsalverschiebung, nach beidseitiger Resektion durch Ventralverschiebung der Vorlast zu verzeichnen. Vorlast $200 \mathrm{~N}$; alle Segmentzustände; Standardabweichung in Klammern; Lateralflexion

\begin{tabular}{|c|c|c|c|c|c|}
\hline $\begin{array}{l}\text { Segmentzustand/ } \\
\text { Vorlastposition (mm) }\end{array}$ & 60 dorsal & 40 dorsal & zentral & 40 ventral & 60 ventral \\
\hline intaktes Segment & $\begin{array}{c}334 \quad( \pm 127) \\
156 \%\end{array}$ & $\begin{array}{c}290 \quad( \pm 101) \\
135 \%\end{array}$ & $\begin{array}{c}214 \quad( \pm 65) \\
100 \%\end{array}$ & $\begin{array}{c}174 \quad( \pm 93) \\
81 \%\end{array}$ & $\begin{array}{c}168 \quad( \pm 83) \\
76 \%\end{array}$ \\
\hline $\begin{array}{c}\text { nach Resektion des rechten } \\
\text { Gelenks }\end{array}$ & $\begin{array}{c}262( \pm 127) \\
126 \%\end{array}$ & $\begin{array}{c}214 \quad( \pm 93) \\
116 \%\end{array}$ & $\begin{array}{c}184 \quad( \pm 30) \\
100 \%\end{array}$ & $\begin{array}{c}144 \quad( \pm 72) \\
80 \%\end{array}$ & $\begin{array}{c}200 \quad( \pm 85) \\
94 \%\end{array}$ \\
\hline $\begin{array}{c}\text { Bandscheiben-Bänder- } \\
\text { Präparat }\end{array}$ & & $\begin{array}{c}303( \pm 99) \\
129 \%\end{array}$ & $\begin{array}{c}235 \quad( \pm 60) \\
100 \%\end{array}$ & $\begin{array}{l}127 \quad( \pm 22) \\
57 \%\end{array}$ & \\
\hline
\end{tabular}

\subsubsection{Laterale Verschiebung der Vorlast}

Beim intakten Segment kam es, besonders bei Verschiebung der Vorlast nach lateral um die ersten $10 \mathrm{~mm}$, zu einem Anstieg der Steifigkeit. Nach der ersten Resektion war es ähnlich, sie nahm jedoch bei Verlagerung der Vorlast nach rechts stärker zu als in Gegenrichtung. Die Steifigkeit war insgesamt geringer als zuvor. Beim BandscheibenBänder-Präparat war sie bereits bei zentraler Vorlast höher als im intakten Segment und nahm bei Lateralverschiebung beinahe linear zu (Abbildung 68).

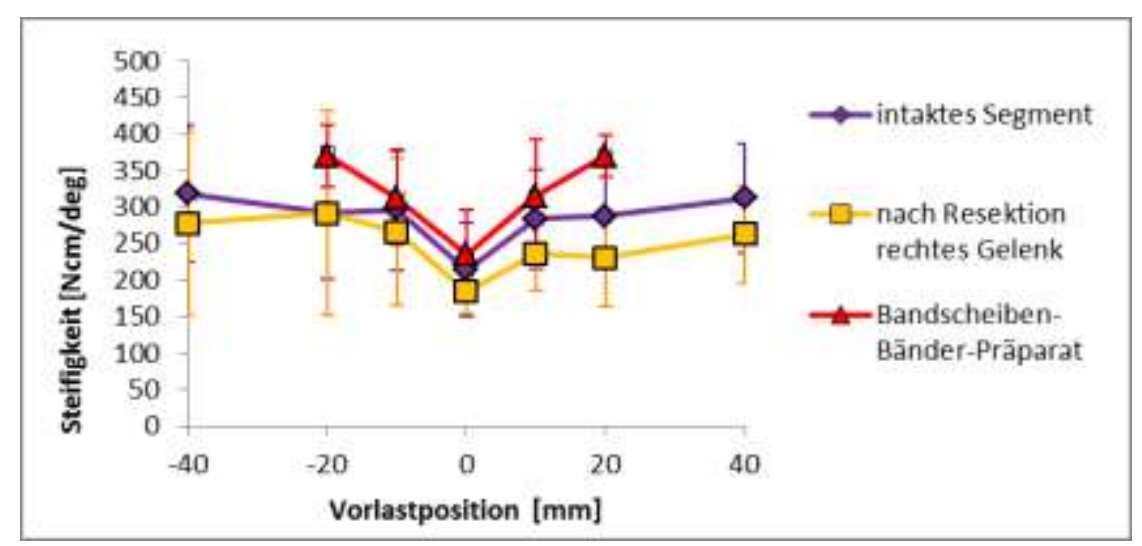

Abbildung 68: Mittlere minimale Steifigkeit in $\mathrm{Ncm} / \mathrm{deg}$ bei allen gemessenen lateralen Vorlastpositionen. Entgegen der anderen Rotationsrichtungen nahm die Steifigkeit beim Experiment „Lateralflexion“ durch Lateralverschiebung der Vorlast zu. Vorlast 200 N; alle Resektionszustände; Lateralflexion

\section{Veränderung der Größe der Vorlast}

Die Steifigkeit verhielt sich umgekehrt proportional zum Range of motion (siehe dort). 


\subsubsection{Zusammenfassung der Ergebnisse: „Lateralflexion“}

Tabelle 19: Zusammenfassung der Ergebnisse des Experiments „Lateralflexion“

Lage und Wanderung der Schraubachse

\begin{tabular}{|c|c|c|}
\hline \multirow{3}{*}{ intaktes Segment } & $\begin{array}{c}\mathrm{VL} \\
\text { zentral }\end{array}$ & $\begin{array}{l}\text { Rastpolkurve verläuft am unteren Bandscheibenrand während der Rechts- } \\
\text { neigung von links nach rechts }\end{array}$ \\
\hline & $\begin{array}{l}\mathrm{VL} \\
\text { ventral }\end{array}$ & $\begin{array}{l}\text { Rastpolkurve verläuft am unteren Bandscheibenrand (entgegengesetzt zu } \\
\text { zentraler und dorsaler Vorlastposition) während der Rechtsneigung von } \\
\text { rechts nach links }\end{array}$ \\
\hline & $\begin{array}{c}\mathrm{VL} \\
\text { dorsal }\end{array}$ & S. VL zentral \\
\hline \multirow{3}{*}{$\begin{array}{l}\text { nach Resektion } \\
\text { des rechten } \\
\text { Gelenks }\end{array}$} & $\begin{array}{c}\mathrm{VL} \\
\text { zentral }\end{array}$ & Rastpolkurve liegt wie beim intakten Segment bei längerem Kurvenverlauf \\
\hline & $\begin{array}{c}\mathrm{VL} \\
\text { ventral }\end{array}$ & $\begin{array}{l}\text { Rastpolkurve liegt wie beim intakten Segment bei kürzerem Kurvenverlauf } \\
\text { (annähernd ortsfest) }\end{array}$ \\
\hline & $\begin{array}{c}\mathrm{VL} \\
\text { dorsal }\end{array}$ & Rastpolkurve liegt wie beim intakten Segment bei kürzerem Kurvenverlauf \\
\hline \multirow{3}{*}{$\begin{array}{l}\text { Bandscheiben- } \\
\text { Bänder-Präparat }\end{array}$} & $\begin{array}{c}\mathrm{VL} \\
\text { zentral }\end{array}$ & Kurvenverlauf ist nochmal länger als bei vorherigem Resektionsschritt \\
\hline & $\begin{array}{c}\mathrm{VL} \\
\text { ventral }\end{array}$ & $\begin{array}{l}\text { Länge der Kurve ist wie bei einseitig reseziertem Segment. Kurvenverlauf } \\
\text { ist entgegengesetzt zum intakten Segment, also während der Rechtsnei- } \\
\text { gung von links nach rechts }\end{array}$ \\
\hline & $\begin{array}{c}\mathrm{VL} \\
\text { dorsal }\end{array}$ & Kurvenverlauf ist länger als bei vorherigem Resektionsschritt \\
\hline
\end{tabular}

\section{Ausrichtung der Schraubachse}

\begin{tabular}{|c|c|c|}
\hline \multirow{3}{*}{ intaktes Segment } & $\begin{array}{c}\text { VL } \\
\text { zentral }\end{array}$ & $\begin{array}{l}\text { Schraubachse verläuft in Rechtsneigung von ventral-links nach dorsal- } \\
\text { rechts und in Linksneigung andersherum }\end{array}$ \\
\hline & $\begin{array}{l}\text { VL } \\
\text { ventral }\end{array}$ & $\begin{array}{l}\text { Schraubachsen verlaufen entgegengesetzt zu zentraler VL, also in Rechts- } \\
\text { neigung von ventral-rechts nach dorsal-links }\end{array}$ \\
\hline & $\begin{array}{c}\mathrm{VL} \\
\text { dorsal }\end{array}$ & s. VL zentral (intaktes Segment) \\
\hline \multirow{3}{*}{$\begin{array}{c}\text { nach Resektion } \\
\text { des rechten Ge- } \\
\text { lenks }\end{array}$} & $\begin{array}{c}\text { VL } \\
\text { zentral }\end{array}$ & $\begin{array}{l}\text { Im Vergleich zum intakten Segment ist die Achsneigung in Rechtsneigung } \\
\text { vermindert }\end{array}$ \\
\hline & $\begin{array}{c}\text { VL } \\
\text { ventral }\end{array}$ & $\begin{array}{l}\text { Im Vergleich zum intakten Segment ist die Achsneigung in Linksneigung } \\
\text { vermindert }\end{array}$ \\
\hline & $\begin{array}{c}\mathrm{VL} \\
\text { dorsal }\end{array}$ & s. VL zentral (nach Resektion des rechten Gelenks) \\
\hline $\begin{array}{l}\text { Bandscheiben- } \\
\text { Bänder-Präparat }\end{array}$ & & $\begin{array}{l}\text { Das Ausmaß der Achsabweichung ist im Vergleich zum intakten Segment } \\
\text { vermindert }\end{array}$ \\
\hline
\end{tabular}

\section{Steifigkeit}

Unabhängig vom Resektionszustand steigt die Steifigkeit bei Verschiebung der Vorlast nach dorsal und sinkt bei Verschiebung nach ventral. Eine extreme Ventralverschiebung führt zu leichtem Anstieg der Steifigkeit 


\subsection{Zusammenfassung wichtiger Ergebnisse}

Die Ergebnisse der Messungen unter Axialrotation zeigten eine Abhängigkeit der Position der momentanen Schraubachsen sowohl von der Vorlastposition als auch vom Resektionsstatus. So verlief die Wanderung der Achse bei ventralen Vorlasten von einem Gelenk über die Bandscheibenmitte zum anderen, während sie bei dorsal gelegenen Vorlasten im gesamten Bewegungsablauf im dorsalen Bandscheibenbereich zu finden war. Wurde das rechte Zwischenwirbelgelenk reseziert, wanderte die Rotationsachse bei zentraler Vorlastposition und bei Drehwinkeln $\alpha<0^{\circ}$ (Rechtsrotation) ähnlich den unresezierten Präparaten von der Bandscheibe in Richtung des verbliebenen Gelenks. Bei Rotationen mit $\alpha>0^{\circ}$ (Linksrotation) hingegen fand eine geringere Wanderung der Schraubachsen in den links-zentralen Bandscheibenbereich statt. Nach den Resektionen konzentrierte sich die Achse unabhängig von der Vorlastposition im mittleren Bandscheibenabschnitt. Im Gegensatz dazu war die Ausrichtung der Schraubachsen besonders durch die Vorlastposition und weniger durch die Resektion der Gelenke zu beeinflussen. Entsprechend lag bei ventralen Vorlasten eine Neigung nach dorsal sowie nach lateral entgegen der Rotationsrichtung (also bei Rechtsdrehung nach links und vice versa) vor. Bei dorsalen Vorlastpositionen war die Achse nach ventral und lateral in Rotationsrichtung geneigt. Durch die Resektion des rechten Zwischenwirbelgelenks konnte nur bei dorsaler Vorlastposition unter Rechtsrotation eine deutliche Veränderung der Achslage - im Sinne eines verminderten Neigungswinkels - festgestellt werden. Nach beidseitiger Gelenkresektion war die Abweichung von der Drehmomentachse insgesamt verringert. Auch die Segmentsteifigkeit zeigte sich abhängig von der Positionierung der Vorlast: Sie stieg mit zunehmender Dorsalverschiebung an und sank bei Ventral- und Lateralverschiebung ab. Jedoch zeigten sich Abweichungen von diesem Verhalten bei Positionen um den Wirbelkanal herum. Es konnte festgestellt werden, dass eine Verschiebung der Vorlast vom Zentrum des Kanals bis $5 \mathrm{~mm}$ nach dorsal zu einem deutlichen Absinken der Steifigkeit führte, bei weiterer Dorsalverschiebung stieg sie dann stetig wieder an. Dieser Effekt konnte besonders deutlich am intakten Segment dargestellt werden, obwohl die Gesamtsteifigkeit nach jedem Resektionsschritt verringert war. Das Bewegungsausmaß verhielt sich stets reziprok zur Segmentsteifigkeit.

Die Versuche in Flexions-Extensionsbewegung zeigten eine deutliche Beeinflussbarkeit der Position der Schraubachsen, während die Ausrichtung weitgehend parallel zur $y$-Achse blieb. So führte einerseits die Verschiebung der Vorlast von ventral nach dorsal zu einer zunehmenden Verlagerung der Rotationsachse vom am unteren Bandscheibenrand gelegenen sagittalen Wirbelkörpermittelpunkt nach dorsal und kaudal, also in Richtung der Wirbelbogengelenke. Andererseits konnte besonders in Extensions-, aber auch in Neutralstellung, eine dorso-kaudale Wanderung der Schraubachse im Verlauf einer Extensionsbewegung aufgezeichnet werden. Der Verlauf der Achsbewegung nach Resektion des rechten Gelenks war ähnlich wie beim intakten Segment. Es konnte lediglich ein Versatz der Rotationsachse nach dorsal bei zentraler und dorsaler Vorlastposition festgestellt werden. An den Bandscheiben-Bänder-Präparaten fiel 
eine kürzere Wanderungsstrecke bei sonst ähnlichem Wanderungsverlauf auf. Die Segmentsteifigkeit zeigte sich auch in dieser Bewegungsabfolge wie zuvor abhängig von der Vorlastposition und dem Resektionszustand. Allerdings verhielt sich der Steifigkeitsverlauf linearer als in Axialrotation. Eine Besonderheit konnte bei lateraler Vorlastverschiebung dargestellt werden. Hier kam es nach Resektion beider Zwischenwirbelgelenke zu einem Anstieg der Steifigkeit bei Vorlastpositionen außerhalb des Wirbelkanals. Dagegen führte dies bei den intakten und einseitig resezierten Segmenten zu einem Steifigkeitsverlust. In Bezug auf die Ausrichtung der Schraubachse konnten weder durch Variation der Vorlast noch durch Resektion der Zwischenwirbelgelenke signifikante Veränderungen hervorgerufen werden.

Auch im Experiment „Lateralflexion“ konnte die Abhängigkeit der Position der momentanen Schraubachsen von der Vorlastposition nachgewiesen werden. Wurde die Vorlast der intakten Segmente zentral positioniert, verlief die Achswanderung am unteren Bandscheibenrand von links nach rechts während der Rechtsneigung und entsprechend von rechts nach links während der Linksneigung. Eine ventrale Verschiebung der Vorlast führte zu einer verringerten Wanderungsstrecke und zur Umkehr der Wanderungsrichtung. Demnach wanderte die Schraubachse nun während der Rechtsneigung von rechts nach links. Dorsale Vorlastpositionen hingegen resultierten in einer verstärkten Wanderung in Richtung der Bogengelenke bei unveränderter Wanderungsrichtung. Auch die Resektion des rechten Zwischenwirbelgelenks führte zu Unterschieden in der Wanderungsstrecke: Während die Strecke bei zentraler Vorlast verlängert war, verkürzte sie sich bei ventralen und dorsalen Vorlastpositionen. In Flexionsstellung konnte zudem annähernde Ortsfestigkeit der Rotationsachse dargestellt werden. Beim beidseitig resezierten Segment verliefen die Schraubachsen bei ventralen Vorlasten ebenfalls etwa ortsfest. Dahingegen lag bei zentraler und dorsaler Vorlast eine ausgeprägte Wanderung der momentanen Schraubachsen mit konstanter Wanderungsgeschwindigkeit vor. Die Wanderungsrichtung verhielt sich nun einheitlich, also fand unabhängig von der Position der Vorlast während der Rechtsneigung eine Wanderung von links nach rechts statt. Anders als bei den Messungen in Axialrotation und Flexion-Extension wurde nun, in Lateralflexion, auch die Ausrichtung der Achsen maßgeblich von der Vorlastposition bestimmt. Dabei unterschied sich die ventrale Position deutlich von der zentralen (im Wirbelkanal) und dorsalen. Während unter erstgenannter Versuchsbedingung eine Neigung der Achsen nach links bei Linksneigung von L3 stattfand, verhielt es sich bei zentraler und besonders dorsaler Vorlast umgekehrt. Nun verkippten die momentanen Schraubachsen bei Linksneigung von L3 nach rechts. Durch die Resektionen des rechten und im Anschluss auch des linken Wirbelbogengelenks wurde das Ausmaß der Neigung vermindert. Dies betraf insbesondere die Achsabweichung in Rechtsneigung bei zentraler Vorlast sowie in Linksneigung bei ventraler Vorlast jeweils nach einseitiger Gelenkresektion. Die Segmentsteifigkeit verhielt sich nach bekanntem Muster. Sie stieg, je dorsaler die Vorlast installiert wurde. Abweichend von den anderen Bewegungsrichtungen führte nun jedoch auch eine Lateralverschiebung zur Erhöhung der Segmentsteifigkeit. 


\section{Diskussion}

Im Folgenden werden zunächst die Bewegungsabläufe der verschiedenen Rotationsrichtungen - axiale Rotation, Ventralflexion-Dorsalextension und Lateralflexion - separat diskutiert. Dabei entspricht die schriftliche Aufarbeitung chronologisch nicht der experimentellen Reihenfolge. Vielmehr verläuft sie retrograd, d.h. der Abschnitt beginnt jeweils mit dem Bandscheiben-Bänder-Präparat, fährt mit dem Zustand nach Resektion des rechten Wirbelbogengelenks fort und endet mit dem intakten Präparat. Damit soll das Verständnis für die Kinematik der Lendenwirbelsäule Schritt für Schritt aufgebaut werden.

\subsection{Axiale Rotation}

\subsubsection{Bandscheiben-Bänder-Präparat}

Die Rastpolkurven, d.h. die Ansammlung der Durchstoßpunkte der wandernden Rotationsachse durch die Horizontalebene auf Höhe der Bandscheibe, fanden sich während der axialen Rotation ortsfest und nahezu mittig in der Bandscheibe. Da diese Position der IHA unabhängig von der sagittalen oder lateralen Verschiebung der Vorlast und dem Rotationsumfang war, spricht dies gegen eine führende Rolle der Bandstrukturen und Materialeigenschaften der Bandscheibe auf den Standort der Schraubachsen.

Hingegen ließen sich im Hinblick auf die Achsausrichtung deutliche Muster erkennen. Bei zentralen und dorsalen Vorlastpositionen lag eine ventrale Neigung der Achsen vor, bei Verschiebung nach ventral verkippten sie zunehmend nach dorsal. Dabei wurde der Nullpunkt bei Vorlastpositionen zwischen $20 \mathrm{~mm}$ und $40 \mathrm{~mm}$ ventral durchwandert, dort, wo auch die Rastpolkurven lagen. Das bedeutet, dass die IHA vertikal verlaufen, wenn die Vorlast in Verlängerung dieser Achse angebracht wird. Eine Verschiebung der Vorlast aus dem Widerstandszentrum heraus führt zur Neigung des oberen Wirbels eines Segments (s. Abschnitt 1.3.4). Die anatomische Lage der entsprechenden Strukturen legt die Vermutung nahe, dass bei ventralen Vorlasten das hintere Längsband sowie die Ligamenta interspinalia und flava sowie bei dorsalen Vorlasten das vordere Längsband auf Zug belastet werden. Möglicherweise könnte diese Bandspannung unter den genannten Versuchsbedingungen zu einer Neigung der momentanen Schraubachsen in Richtung der gedehnten Bandstrukturen führen. Auch innerhalb eines Rotationsdurchlaufs änderte sich der Neigungswinkel der Achsen, besonders in lateraler Richtung. Dieser Effekt beruht nach Mansour (2001) auf den elastischen Eigenschaften der Bandscheibe. Demnach befinde sich diese nach axialer Druckbelastung durch die Vorlast in einem labilen Gleichgewicht, ähnlich einem in Längsrichtung komprimierten Gummistab. Wirke nun eine weitere Kraft, z.B. das axiale Drehmoment, werde das elastische Objekt verdrillt und das System gerate aus dem 
Gleichgewicht, der Wirbelkörper kippe nach lateral. Die Richtung der Neigung werde dabei durch die elastischen Eigenschaften des Discus intervertebralis und der Bandstrukturen determiniert.

Die Segmentsteifigkeit wird durch unterschiedliche Mechanismen bestimmt. Zunächst seien die reinen Bandscheibeneigenschaften (ohne Bogengelenke) erwähnt. Ein zur z-Achse paralleles und im Widerstandszentrum angelegtes Drehmoment führt zur axialen Rotation und somit zur reinen Torsion der Bandscheibe. Hierdurch übt der Anulus fibrosus durch seinen scherengitterartigen Faserverlauf Kompression auf den gallertigen Nucleus pulposus des Discus intervertebralis aus (Bogduk und Mercer 2000). Die Bandscheibe nimmt an Höhe zu, was wiederum zur Ausbildung eines rücktreibenden Drehmoments und somit zur Zunahme der Torsionssteifigkeit beiträgt (Nägerl et al. 1995). Dies trägt als ein Faktor zur Erklärung der Parabelform der Steifigkeitskurve bei. Denn je stärker das Rotationsausmaß war, desto höher auch die Steifigkeit. In Neutralstellung $\left(\alpha=0^{\circ}\right)$ fiel sie dementsprechend am geringsten aus. Zusätzlich könnte auch die Verkippung der Wirbel gegeneinander den Anstieg der Rotationssteifigkeit unterstützen: Unter der o.g. Vermutung, dass durch die Bewegung des oberen Wirbels Bandstrukturen ebenso wie die Bandscheibe selbst auf Zug bzw. Stauchung belastet werden, würden durch die elastischen Eigenschaften Rückstellkräfte aktiviert, die zur Erhöhung der Segmentsteifigkeit beitragen.

Liegt das Rotationszentrum außerhalb des Widerstandszentrums oder sind auch Zwischenwirbelgelenke an der Bewegung beteiligt, kommt ein zusätzlicher Aspekt hinzu, der im Abschnitt 4.1.3 erläutert wird.

\subsubsection{Segment nach Resektion des rechten Zwischenwirbelgelenks}

Die Messungen an den einseitig resezierten Präparaten lieferten Ergebnisse, die eindeutig auf eine Führungsfunktion der Gelenke hinweisen. So lagen die Rastpolkurven nun in der linken Bandscheibenhälfte und verliefen je nach Vorlastposition im Bereich zwischen dem Discus intervertebralis und dem verbliebenen Bogengelenk.

Bei zentraler Vorlast fand bei Drehwinkeln $\alpha<0^{\circ}$ (Rechtsrotation) eine Wanderung der Schraubachsen vom eher ventralen und mittigen Startpunkt in die dorso-laterale Bandscheibe, also in Richtung des Gelenks, statt. Da diese Wanderungsbewegung beim Bandscheiben-Bänder-Präparat nicht stattgefunden hat, lässt sich vermuten, dass das Gelenk in diesem Bewegungsabschnitt eine Führung der Bewegung übernimmt. Möglich ist dies, da sich mit zunehmendem Rotationswinkel die Gelenkfortsätze aufeinander zubewegen und die Gelenkflächen kraftschlüssig werden (Wachowski et al. 2009). Die Krümmungs-Inkongruenz der an der Bewegungsführung beteiligten Gelenkflächen ermöglicht ein Abrollen der Facetten aneinander. Das Gelenk fungiert als Hypomochlion und die Rastpolkurve bewegt sich mit zunehmender Rotation zum führenden Gelenk hin. Es greift das Prinzip der dimeren Gelenkkette (Nägerl 1990). Findet allerdings eine Linksrotation statt, verblieb die Rastpolkurve im mittleren linken Bandscheibenbereich. Dies kann dadurch erklärt werden, dass sich die Gelenkflächen des linken Gelenks voneinander entfernen und die Führung durch das Gelenk bei $\alpha \approx 0^{\circ}$ aufgehoben 
wird. Dabei ist die tendenziell linksseitige Lage am ehesten darauf zurückzuführen, dass die Gelenkkapsel um das verbliebene Gelenk auch bzw. gerade bei fehlendem Kontakt der Gelenkflächen gespannt ist und somit zur lateralen Verlagerung der IHA führt.

Der höhere Rollanteil der Gelenkflächen und die damit verbundene Dezentralisierung der momentanen Schraubachsen nehmen wiederum Einfluss auf die Rotationssteifigkeit des Segments. Da die IHA und somit auch das Rotationszentrum nun außerhalb der Bandscheibe liegen, kommt es mit zunehmender Rotation zur wachsenden Translation der Wirbel. Dieser Scherkraft setzt das Zwischenwirbelgelenk als Hypomochlion eine Konterkraft entgegen, so dass ein Kräftepaar aus Scherkraft und Gelenkkraft entsteht. Dieses bildet nun ein Gegendrehmoment $T_{G}$ zum axial wirkenden Drehmoment $T_{Z}$, das durch die Messapparatur angelegt wird. Das Bewegungsausmaß des Segments verhielt sich - wie schon im Bandscheiben-Bänder-Präparat - reziprok zur momentanen Steifigkeit.

\subsubsection{Intaktes Segment}

In den Versuchen der intakten Segmente kann die Auswirkung des Kopplungs- bzw. Entkopplungsmechanismus der Zwischenwirbelgelenke durch sagittale Vorlastverschiebung (Flexions- bzw. Extensionsstellung des Segments) auf die Rastpolkurve dargestellt werden. Zum leichteren Verständnis wird dabei zunächst auf die Biomechanik der Gelenke in Flexions- und Extensionsstellung eingegangen.

In Flexionsstellung weisen die Ergebnisse der Experimente auf eine Ein-GelenkFührung im Sinne der dimeren Gelenkkette hin. Bei nichtrotiertem Segment, also wenn $\alpha=0^{\circ}$, liegen beide Zwischenwirbelgelenke durch die ventrale Vorlast entkoppelt vor (Wachowski et al. 2009). Doch schon bei geringsten Drehwinkeln wird eines der Gelenke kraftschlüssig und die momentanen Schraubachsen wandern direkt bis zum führenden Gelenk. Auffällig war unter diesen Versuchsbedingungen der Versatz der gesamten Rastpolkurve nach dorsal, ein Effekt, der auch im Segment L1/L2 aufgetreten ist (Wachowski et al. 2010b). Entsprechend der Theorie der Gelenkentkopplung muss die Rotationsachse bei $\alpha \approx 0^{\circ}$ in der Nähe des Bandscheibenzentrums liegen (Kinematik wie im Bandscheiben-Bänder-Präparat). Eine mögliche Erklärung wäre die gesteigerte Kapselspannung und Dehnung des hinteren Längsbandes, die bei Flexion an den Zwischenwirbelgelenken entstehen und durch eine scharnierartige Wirkung eine dorsale Lage der Rastpolkurve bedingen.

Wurde das Segment durch dorsale Vorlasten in Extensionsstellung gebracht, wanderte die Rastpolkurve bogenförmig. Ein solcher Kurvenverlauf kann unter den Voraussetzungen für eine symmetrische Vier-Gelenk-Kette erwartet werden (Nägerl 1990, Kubein-Meesenburg et al. 1991a, Nägerl et al. 1992). Auf ein Wirbelsäulensegment übertragen, beruht dieses Konzept auf dem gleichzeitigen Gelenkschluss beider Zwischenwirbelgelenke. Daher kann davon ausgegangen werden, dass während der gesamten Rotation beide Gelenke kraftschlüssig sind. Die Rastpolkurve verlief jedoch nicht, wie nach den Versuchen von Mansour (2001) erwartet, dorsal zwischen den Gelenken, 
sondern nach ventral in der dorsalen Hälfte der Bandscheibe. Diese scheinbare Diskrepanz lässt sich mit folgenden Überlegungen reduzieren: Kommt es in Extensionsstellung beim Kraftschluss zu einem Kontaktpunkt, der im dorsalen Facettenbereich liegt und nicht wie in bisherigen Darstellungen von Mansour im ventralen, resultiert ein nach ventral konvexer Verlauf der Schraubachsen. Weiterhin könnten auch mikroskopische Unebenheiten in der Gelenk-Oberfläche die Wanderbewegung beeinflussen.

In Neutralstellung (zentrale Vorlast) hingegen kann eine Zwischenstufe zwischen reiner Ein- und Zwei-Gelenk-Führung vermutet werden. Bei einem Drehwinkel $\alpha \approx 0^{\circ}$ befanden sich die Schraubachsen zunächst in der Nähe des Bandscheibenzentrums. Da dies der Bewegungsstruktur von Bandscheiben-Modell und Bandscheiben-BänderPräparat entspricht - es waren jeweils keine Gelenke vorhanden - kann auch im intakten Segment von einem momentanen Zustand ohne Gelenkführung ausgegangen werden. Wird die Rotation größer, beispielsweise nach rechts, nähern sich die Gelenkflächen des linken Zwischenwirbelgelenks zunehmend an, werden kraftschlüssig und das Gelenk übernimmt die Führung (Wachowski et al. 2009). Dies könnte die Wanderung der Rastpolkurve zum linken Gelenk erklären. Da aber, bedingt durch die zentrale Vorlast und die Inkongruenz der Facetten, auch auf der rechten Seite Gelenkflächenkontakt entsteht, verlief die Kurve leicht bogenförmig und endete dorsal der Bandscheibe. Es kann also von einer Zwei-Gelenk-Führung ausgegangen werden, bei der jedoch im Unterschied zum extendierten Segment in Abhängigkeit vom Rotationsausmaß die Führung eines der Gelenke überwiegt.

Durch laterale Positionierung der Vorlast konnte ein unterschiedliches Wanderverhalten der IHA während Links- bzw. Rechtsrotation festgestellt werden. Lag die Vorlast beispielsweise rechts, wanderte die Rastpolkurve in der Linksrotation $\left(\alpha>0^{\circ}\right)$ zum rechten Gelenk und war somit vergleichbar mit dem Verlauf bei zentraler Vorlast. Die Kurve bei Rechtsrotation hingegen ähnelte derjenigen bei dorsaler Vorlast. Nimmt man nun an, dass ein ähnliches Kopplungsverhalten der Gelenke einen ähnlichen Kurvenverlauf hervorruft, sprechen die Ergebnisse gemäß der vorangegangen Überlegungen für einen Wechsel zwischen Ein-Gelenk-Führung des rechten Gelenks bei linksrotiertem Segment und Zwei-Gelenk-Führung bei rechtsrotiertem Segment. Diesem Wechsel könnte folgender Mechanismus zugrunde liegen: Durch rechts-laterale Vorlasten neigte sich der obere Wirbel makroskopisch sichtbar nach rechts. Diese Bewegung könnte möglicherweise einen Gelenkschluss der Facetten des rechten Gelenks bewirken, während das linke entkoppelt wird. Findet nun eine Drehung nach links statt, würde die Kopplung des rechten bzw. Entkopplung des linken Gelenks beibehalten oder sogar verstärkt, so dass lediglich das rechte Gelenk die Führung der Bewegung übernimmt. Die Rotation nach rechts hingegen könnte zu einer rotationsbedingten Kopplung des linken Gelenks führen, während das rechte Gelenk aufgrund der lateralen Vorlast kraftschlüssig bliebe. In diesem Fall ginge die Führung von beiden Gelenken aus.

Bei Betrachtung der Ausrichtung der Schraubachsen wird ebenfalls ein Muster erkennbar. Konzentriert man sich zunächst auf die Neutralstellung (Drehwinkel $\alpha=0^{\circ}$ ) fällt 
auf, dass bei zentraler und besonders dorsaler Vorlast eine Achsneigung nach ventral vorlag, bei ventraler Vorlast jedoch nach dorsal. Man könnte die These postulieren, Kraftschluss der Gelenke ginge mit ventraler Ausrichtung der IHA einher. Dies kann jedoch widerlegt werden, da auch nach Resektion der Bogengelenke dieses Muster zu sehen war, wenn auch mit kleineren Neigungswinkeln. Weiterhin blieb die ventrodorsale Neigung auch während der Rotation weitgehend konstant, obwohl, je nach vorlastbedingtem Flexions- bzw. Extensions- und Rotationsausmaß, unterschiedliche Gelenkführungszustände vorlagen. Für die laterale Abweichung der Achsen gelten ähnliche Überlegungen. Bei $\alpha=0^{\circ}$ verliefen die Schraubachsen unabhängig von der Vorlastposition meist parallel zum einwirkenden Drehmoment. In den Rotationsmaxima kam es zu einer deutlichen Abweichung. Diese fand bei zentralen und dorsalen Vorlasten in Rechtsrotation nach links und umgekehrt in Linksrotation nach rechts statt. Vorflektierte Segmente verhielten sich entgegengesetzt, sind also in Rechtsdrehung nach rechts, in Linksdrehung nach links geneigt. Diese Tendenzen blieben auch nach $\mathrm{Re}-$ sektion der Zwischenwirbelgelenke erhalten und können daher nicht auf diese zurückgeführt werden. Vielmehr liegt stattdessen der Schluss nahe, dass die Ausrichtung der momentanen Schraubachsen von der Struktur und dem Zusammenspiel von Bandscheibe und Bändern festgelegt und von den Gelenken unterstützt wird. Insgesamt ist nach Resektion der Gelenke die Ausprägung der Neigung bei gleichbleibender Tendenz der Neigungsrichtung sehr variabel. Dies spricht in diesem Kontext zudem für eine Funktion der Wirbelbogengelenke in Bezug auf die Stabilisierung der Achsausrichtung.

Bei der Betrachtung der Segmentsteifigkeit des intakten Segments konnten Zusammenhänge mit der Position der Vorlast einerseits und dem Verlauf der Rastpolkurven andererseits festgestellt werden. So lag bei flektiertem Segment (ventrale Vorlastposition) unabhängig von der Bewegungsrichtung eine geringere Steifigkeit vor. Mit zunehmender Extension stieg sie an. Unter der Annahme, dass durch die Position der Vorlast das Ausmaß des Gelenkschlusses variiert werden kann, ließe sich aufgrund der Ergebnisse eine Beeinflussung der Segmentsteifigkeit durch eine Gelenkführung vermuten. Untermauert wird diese These durch die Resektionsversuche. Denn bereits nach der Resektion eines, besonders jedoch beider Zwischenwirbelgelenke, sank die Gesamtsteifigkeit der Segmente deutlich. Weiterhin konnte am Bandscheiben-BänderPräparat auch die Abhängigkeit der Steifigkeit von der Vorlastposition und folglich vom Kraftschluss der Gelenke nicht mehr in der Deutlichkeit dargestellt werden. Möglicherweise fungieren kraftschlüssige Gelenke als Widerlager und führen zu einer Translation des oberen Wirbels gegenüber dem unteren. Dadurch entsteht ein Gegendrehmoment, das die Steifigkeit steigert. Nach Wachowski et al. (2010a) ist die Steifigkeit höher, je weiter das Rotationszentrum vom Widerstandszentrum entfernt liegt, da infolgedessen auch das Ausmaß der Scherbewegung steigt. Dieser Mechanismus greift auch bei wandernden Schraubachsen und kann somit auf die Rastpolkurve angewendet werden. 
Weiterhin ist beim Vergleich von Steifigkeit und sagittaler Schraubachsausrichtung eine Korrelation gegeben. Dorsale Neigungswinkel gingen mit einer geringeren, ventrale Winkel mit einer erhöhten Steifigkeit einher. Allerdings liegt dieser Beobachtung vermutlich keine kausale Beziehung zugrunde. Denn während die erhöhte Segmentsteifigkeit bei extendiertem Segment wahrscheinlich auf die Interaktion der Zwischenwirbelgelenke mit der Bandscheibe zurückzuführen ist, resultiert die ventrale Ausrichtung der IHA am ehesten aus dem gestrafften vorderen Längsband. Eine differenziertere Betrachtung folgt im Zusammenhang mit dem singulären Steifigkeitsanstieg (s. Abschnitt 0)

Das Bewegungsausmaß (ROM) verhielt sich reziprok, stieg also, je geringer die Steifigkeit war. Dabei zeigen die Ergebnisse zur Axialrotation, dass die Steifigkeit das sensiblere Maß ist. So stieg der ROM bei Vorlastverschiebung von zentral auf 2,5 mm dorsal um lediglich $13 \%$, während die Steifigkeit um $32 \%$ sank. Unterschiede in der Steifigkeit führten also nicht unbedingt zu vergleichbaren Veränderungen im Bewegungsausmaß.

Das Verhalten der Hysterese legt den Schluss nahe, dass mit Erreichen des Kraftschlusses der Articulationes zygapophysiales die viskös-elastischen Eigenschaften der Bandscheibe im Bewegungsablauf eine zunehmend untergeordnete Rolle spielen. Entsprechend werden diese Eigenschaften relevanter, je mehr Gelenk- und Bänderanteile reseziert werden.

Interessanterweise kann die Höhe der Vorlast, also das Ausmaß der axialen Belastung, im Versuchsaufbau „axiale Rotation“ nicht als relevanter Einflussfaktor auf den Kontaktschluss der Gelenkfacetten dargestellt werden. Es wurden zwar geringe Auswirkungen auf die Position der Ratspolkurve gefunden, jedoch zeigten sich diese unabhängig vom Resektionsausmaß. Weiterhin variierte der Kurvenverlauf individuell. Allerdings war dieser Aspekt kein Schwerpunkt dieser Arbeit.

\subsection{Ventralflexion-Dorsalextension}

\subsubsection{Bandscheiben-Bänder-Präparat}

Im Gegensatz zur axialen Rotation konnte beim Bandscheiben-Bänder-Präparat nach Anlage eines Drehmoments parallel der y-Achse eine Abhängigkeit der Position der momentanen Schraubachsen von der Lage der Vorlast nachgewiesen werden. Die dorsale Lage der IHA bei dorsalen Vorlastpositionen scheint zunächst im Widerspruch zu dem Mechanismus zu stehen, der bisher diskutiert wurde. Denn wäre auch hier das Wechselspiel zwischen Anspannung und Entspannung der Längsbänder für die Achsposition verantwortlich, würde bei dorsalen Vorlastpositionen ein ventral gerichteter Ortswechsel erwartet. Die Ursache für dieses atypische Verhalten liegt aber möglicherweise in einem unphysiologischen Aufeinandertreffen der Processi spinosi: Durch die fehlende Stützfunktion der resezierten Zwischenwirbelgelenke nähern sich die Dornfortsätze bei zunehmender Extension an und bilden akzidentiell eine gelenkartige Ver- 
bindung, die wiederum die Lage der Durchstoßpunkte der Schraubachsen bestimmt. Mit steigender Flexionsbewegung verlieren die Processi zwar den Kontakt, gleichzeitig wird aber vermutlich das hintere Längsband zunehmend auf Zug belastet und bahnt somit die dorsale Lage der IHA. Dieser mechanische Vorgang könnte demnach sowohl für die dorsal gerichtete Wanderung der momentanen Schraubachsen während der Extensionsbewegung verantwortlich sein, als auch für die zunehmend dorsale Lage der Rastpole bei dorsalen Vorlastpositionen.

Die Ausrichtung der momentanen Schraubachsen wird am ehesten nach bekanntem Prinzip durch die Anspannung der Bandstrukturen beeinflusst. So wies ein flektiertes Segment dezent nach dorsal geneigte IHA auf, während extendierte Präparate leicht ventral verkippte Achsen zeigten. Dabei scheint es unerheblich, ob die Flexion bzw. Extension durch die Position der Vorlast bestimmt oder durch Anlage des Drehmoments erreicht wird.

Die Betrachtung der Segmentsteifigkeit und somit auch des Bewegungsausmaßes weist wiederum darauf hin, dass bei resezierten Segmenten das Spannungsmaß der Ligamenta einen potenten Mechanismus darstellen könnte. Dabei wird dieser Spannungszustand nicht nur durch die sagittale oder laterale Vorlastposition bestimmt, sondern zusätzlich durch den Bewegungszeitpunkt. So führt die Ventralflexion vermutlich zu einer zunehmenden Dehnung der dorsalen Bandanteile - nach Adams und Hutton (1980) und Silver (1954) inklusive der Ligamenta inter- und supraspinalia - wodurch die Steifigkeit erhöht wird. Reziprok geht der Einfluss in Dorsalextension von den ventralen Bandstrukturen aus.

Eine weitere Möglichkeit zur Modulation der Segmentsteifigkeit wird im Abschnitt 4.2.3 erläutert.

\subsubsection{Segment nach Resektion des rechten Zwischenwirbelgelenks}

Die im Vergleich zum Bandscheiben-Bänder-Präparat kaudalere Lage der Rastpolkurven nach Resektion eines Gelenks könnte auf die Gelenkführung zurückgeführt werden. Bestärkt wird diese Vermutung dadurch, dass besonders bei zentralen und dorsalen Vorlastpositionen, in denen das Gelenk gemäß der bereits diskutierten Theorie kraftschlüssig wird, die beschriebenen qualitativen Effekte deutlich werden. Der Mechanismus des Kraftschlusses ist derselbe, wie in Abschnitt 4.1 beschrieben. Ebenso gilt das bereits erläuterte Konzept der dimeren Gelenkkette (Nägerl et al. 1990). Im Unterschied zur axialen Rotation findet nun jedoch aufgrund der Morphologie der Gelenkfacetten und der Asymmetrie des gesamten Segments am ehesten eine kombinierte Roll-Gleitbewegung statt, die die beschriebene Wanderung der IHA determiniert. Im Einklang dazu steht die erhöhte Steifigkeit bzw. der verminderte ROM, der bei Kraftschluss der Gelenke auftritt.

Auffallend ist jedoch, dass die Veränderungen der IHA stärker ausgeprägt waren als am intakten Segment (s. Abschnitt 4.3). Eine mögliche Ursache könnte in der Asymmetrie liegen, die durch die einseitige Resektion entsteht. Demnach würde die Aus- 
übung einer Kraft auf das Segment zu einer Rotation und Neigung des oberen Wirbels führen (s.u.) und das verbliebene Gelenk würde nicht physiologisch belastet. D.h. es entstünden differente Kontaktpunkte der Gelenkfacetten, was wiederum die Position der momentanen Schraubachse beeinflusst.

Die Rolle der Bogengelenke für die Ausrichtung der IHA scheint auch in der FlexionsExtensions-Bewegung eher untergeordnet zu sein. Die größten Abweichungen fanden sich nach Resektion des rechten Gelenks. In diesem Fall verliefen die Achsen in Neutralstellung von rechts-ventral nach links-dorsal. Dies ist verständlich, da die Stützfunktion durch das Gelenk und die Gelenkkapsel auf der rechten Seite fehlt. Zusätzlich fungiert das verbliebene linke Articulatio wie bereits diskutiert als Widerlager. Dadurch erfährt der obere Wirbel (L3) ein nach rechts gerichtetes Drehmoment und die linke Gelenkkapsel gerät unter Zugspannung. Dies könnte die Neigung der momentanen Schraubachsen in Richtung des verbliebenen Gelenks zur Folge haben. Die vertikale Abweichung war nur gering und unterlag starken interindividuellen Unterschieden, die durch die Gelenkresektionen verstärkt wurden. Insgesamt wird die These der unterstützenden Gelenkfunktion durch diese Befundlage bestärkt.

\subsubsection{Intaktes Segment}

Die vorlastbedingte Flexion bzw. Extension führte zu einer Verlagerung der momentanen Schraubachsen nach ventral bzw. dorsal. Zusätzlich wanderten sie im Verlauf einer Extensionsbewegung aufgrund der vermuteten zunehmenden Kraftschlüssigkeit der Wirbelgelenke nach dorsal, umgekehrt im Rahmen der Flexion nach ventral. Eine Abweichung besteht bei Flexion mit ventralen Vorlasten, während der es zur Wanderung der Achsen nach dorsal kam. Dies wäre durch die gesteigerte Spannung im hinteren Längsband zu erklären. Sie bedingt möglicherweise die Verlagerung des Rotationszentrums, sichtbar durch Darstellung der IHA, nach dorsal. Wird die Vorlast nach ventral verschoben, verlieren die Gelenkflächen den Kontakt (Schendel et al.1993). Die Rastpolkurve war nun deutlich kürzer als noch bei zentraler Vorlast und lag mittig in der Bandscheibe, da die Bewegung weniger Gelenkführung unterliegt. Auch die Wanderungsgeschwindigkeit unterstreicht das Führungsverhalten der Gelenke. Waren die Rastpole bei den resezierten Präparaten noch relativ ortsfest und wanderten nur in starker Extension nach dorsal, lag im intakten Segment eine gleichmäßige Wanderungsgeschwindigkeit vor. Lediglich bei ventraler Vorlastposition blieben die IHA ortsfest. Diese Beobachtung deckt sich mit der Vorstellung, dass in der vorlastbedingten Entkopplung der Zwischenwirbelgelenke keine Gelenkführung vorliegt.

Bei diesem Segmentzustand deuten die Messergebnisse erneut auf das Vorliegen einer dimeren Gelenkkette hin, wobei nun zwei dieser Ketten parallel in einer Ebene liegen. Im Gegensatz zum einseitig resezierten Segment führt die symmetrisch beidseitige Gelenklage zu einem vergrößerten Roll- und verringerten Gleitanteil an der Bewegung. 
Analog zur axialen Rotation wird die Segmentsteifigkeit auch in der FlexionsExtensionsbewegung durch unterschiedliche Mechanismen determiniert. Bemerkenswert sind in diesem Zusammenhang die Auswirkungen der Veränderung der Vorlastgröße. Je größer die Vorlast gewählt wurde, desto weiter dorsal kamen die Rastpolkurven zu liegen und desto größer wurde die momentane Steifigkeit. Gleichzeitig fand sich eine qualitative Änderung der Form der Drehwinkel-Drehmomentkurve. So ähnelte der Kurvenverlauf bei erhöhter Vorlast derjenigen bei dorsalen Vorlastpositionen, während die Kurve bei verringerter Vorlast der bei ventralen Vorlastpositionen glich. Die Gleichartigkeit der Ergebnisse bei Verschiebung und Erhöhung der Vorlast legt die Vermutung nahe, dass in beiden Fällen derselbe Mechanismus die Segmentsteifigkeit beeinflusst. Da diese Veränderungen unabhängig vom Resektionszustand der Segmente dargestellt werden konnten, ist davon auszugehen, dass der Mechanismus nicht auf eine Gelenkführung zurück zu führen ist. Stattdessen scheint unter den vorliegenden Versuchsbedingungen neben den Materialeigenschaften von Bändern und Bandscheibe (s. Abschnitte 4.1.1 und 4.2.1) besonders die Lage der momentanen Schraubachsen ausschlaggebend zu sein.

\subsection{Lateralflexion}

\subsubsection{Bandscheiben-Bänder-Präparat}

Die Ergebnisse dieser Rotationsrichtung veranschaulichen die Relevanz der Bandstrukturen auf die Stabilität des Wirbelsäulensegments. Die ausgeprägte und konstante Wanderung der momentanen Schraubachsen bei zentraler Vorlast deuten auf das Vorliegen eines instabilen Gleichgewichts hin. Die zunehmende Lateralneigung der IHA in den Rotationsmaxima bestärkt diese Annahme. Bereits geringe Krafteinwirkungen von außen führen zu einer Neigung des kranialen Wirbels, wodurch vermutlich die lateralen Bandscheibenanteile auf Zug bzw. auf Druck beansprucht werden. Entgegen der axialen Rotations- und Flexions-Extensionsbewegung gibt es nun keine Bandstrukturen, die durch laterale Flexion unmittelbar gedehnt werden. Allenfalls kämen hierfür die lateralen Anteile des Ligamentum longitudinale anterius und die Ligamenta intertransversaria in Frage. Letztere haben sich jedoch schon während der Präparation als nicht sehr widerstandsfähig erwiesen. Somit ist verdeutlicht, warum im Gegensatz zu den anderen Bewegungsrichtungen keine Ortsfestigkeit der IHA vorliegt. Diese wurde hingegen mit ventralen Vorlastpositionen erreicht. Denn durch die Vorflexion des Segments könnten nun sowohl das Ligamentum longitudinale posterius als auch die bogen- und spinosi-assoziierten Bandstrukturen gespannt werden und die Wirbel stabilisieren.

\subsubsection{Segment nach Resektion des rechten Zwischenwirbelgelenks}

Die Veränderungen, die in diesem Experiment durch sagittale Verschiebung der Vorlast erzielt wurden, ähnelten im Wesentlichen denen der Versuche des Bandscheiben- 
Bänder-Präparats. Lediglich war die Wanderungsstrecke bei Vorhandensein des rechten Gelenks leicht verkürzt (zentrale und dorsale Vorlastpositionen) bzw. verlief die Kurve annähernd ortsfest (ventrale Vorlastposition). Diese Effekte können auch in dieser Bewegungsrichtung mit der in den vorherigen Abschnitten diskutierten Theorie veranschaulicht werden: In Flexionsstellung des Segments ist das verbliebene Gelenk entkoppelt und nimmt entsprechend unabhängig vom Rotationsausmaß keinen Einfluss auf die ortsfesten IHA. Möglicherweise steht jedoch die Gelenkkapsel unter Spannung und könnte in diesem Falle die leicht zum Gelenk versetzte Position der Schraubachsen bedingen. Bei dorsal liegender Vorlast hingegen wäre das Gelenk bei linksgeneigtem Segment kraftschlüssig. Dadurch weicht die Rastpolkurve von ihrem Verlauf, der vom beidseits resezierten Präparat bekannt ist, ab und bewegt sich zum vorhandenen Gelenk. In Rechtsneigung entkoppelt das Gelenk und es liegen ähnliche Ergebnisse wie unter Abschnitt 4.3.1 beschrieben vor. Die Geringfügigkeit der durch den Resektionsschritt hervorgerufenen Veränderungen weist jedoch darauf hin, dass das verbliebene Gelenk zwar Einfluss auf den Kurvenverlauf nimmt, jedoch nicht den dominanten Führungseffekt besitzt.

Bei Betrachtung der Richtung der Schraubachsen stachen besonders die Messungen bei ventraler Vorlast mit Rechtsneigung sowie zentraler Vorlast mit Linksneigung hervor. Eine mögliche Ursache für diese Auffälligkeiten könnte in einer zusätzlichen axialen Rotationskomponente liegen, die durch die Asymmetrie nach einseitiger Resektion hervorgerufen wird. In der Linksneigung könnte das verbliebene Zwischenwirbelgelenk als Hypomochlion fungieren, in der Rechtsneigung nähme die Gelenkkapsel die Energie auf. In beiden Fällen käme es dadurch zu besagter Rotation. Die erhöhte Steifigkeit, die bei ventralen Vorlastpositionen sehr ungewöhnlich ist, wäre ein weiteres Indiz für diese Theorie.

\subsubsection{Intaktes Segment}

Zur Darstellung des Einflusses der Zwischenwirbelgelenke in Abhängigkeit von der Vorlastposition wird zunächst der Zustand bei entkoppelten Gelenken betrachtet: Bei vorflektiertem Segment (ventrale Vorlast) befanden sich die Rastpolkurven in Neutralstellung in der Nähe des Bandscheibenzentrums. Wurde nun der Wirbel L3 nach rechts geneigt, blieb die Wanderungsbewegung der Rastpolkurven gering, war aber im Vergleich zum vollständig resezierten Präparat vorhanden. Dies wird unter der Annahme nachvollziehbar, dass sich die Gelenkfacetten zwar annähern, aber nicht vollständig kraftschlüssig werden. Die Ergebnisse entsprächen somit dem Modell der überschlagenen dimeren Gelenkkette mit abrollendem Gelenk. Das extendierte Segment hingegen zeigte die Kinematik der Viergelenkkette. Beide Bogengelenke wären also während der Bewegung gekoppelt und führten die Rotation. Dementsprechend verlagerten sich die IHA während der Rotation kontinuierlich in Richtung Gelenkspalt. Dieses Muster zeigte sich auch bei Positionierung der Vorlast im Wirbelkanal, also geringfügig dorsal des Widerstandszentrums. Dies lässt den Schluss zu, dass bereits relativ dezente Extensionen zum Kraftschluss der Wirbelbogengelenke führen. 
Eine Verschiebung der Vorlast vom Spinalkanal nach lateral führte zu einer sichtbaren Neigung von L3, jedoch nicht zur Entkopplung des gegenüberliegenden Gelenks. Zwar war die Rastpolkurve zu jenem Facettengelenk verschoben, auf dessen Seite die Vorlast lag - das spricht für die stärkere Führung dieses Gelenks - jedoch zeigten die Schraubachsen den für die Viergelenkkette typischen Verlauf. Es liegt also unabhängig von der Lateralneigung eine Zwei-Gelenk-Führung vor.

Wie bereits im vorherigen Abschnitt erwähnt, sind die Veränderungen an den Rastpolkurven durch die Resektionen eher quantitativer als qualitativer Natur. Im Vergleich zu den Versuchen „Axialrotation“ und „Ventralflexion-Dorsalextension“ scheint das Führungsverhalten der Zwischenwirbelgelenke also eine untergeordnete Rolle bei der Determination der Rastpolkurven zu spielen.

Im Gegensatz zur axialen Rotation sowie der Flexions-Extensionsbewegung beeinflusst die Vorlastposition in Lateralflexion neben der Position der IHA maßgeblich auch die Ausrichtung der IHA. Während die Schraubachsen in der Frontalebene bei jeglichen Versuchsanordnungen keine Abweichungen von der Drehmomentachse aufwiesen, war die laterale Orientierung sowohl von der Vorlastposition als auch vom Rotationsausmaß bestimmt. Unabhängig vom Resektionszustand waren die momentanen Schraubachsen bei $\alpha=0^{\circ}$ zunächst parallel zur x-Achse. Spezifische Veränderungen fanden dann mit zunehmender Neigung des Wirbels L3 statt. Die Betrachtung der intakten Präparate zeigt erneut die Sonderstellung des vorflektierten Segments (ventrale Vorlastposition). Man sollte in diesem Zusammenhang bedenken, dass im aktuellen Versuchsaufbau sowohl dorsale als auch zentrale Vorlastpositionen dorsal des Widerstandszentrums gelegen sind. Es kann folglich erwartet werden, dass sich auch entsprechend die Ergebnisse dieser Messungen ähneln, die der ventralen Vorlasten jedoch zum Teil deutlich differieren. In letztgenannter Versuchsreihe (ventrale Vorlast) kam es in Linksneigung zu einer Abweichung der IHA nach links, umgekehrt wiesen sie in Rechtsneigung nach rechts. Zentrale und dorsale Vorlastpositionen führten hingegen zur Abweichung der momentanen Schraubachsen nach rechts, wenn der obere Wirbel nach links geneigt war. Dies ist ein Hinweis, dass unter den genannten Versuchsbedingungen in Linksneigung eine Ein-Gelenk-Führung, in Rechtsneigung jedoch eine Zwei-Gelenk-Führung vorliegen könnte. Denn je näher das Rotationszentrum in Richtung des durch die Neigung „belasteten“ Gelenks wandert, desto mehr Scherkraft muss von den gegenüberliegenden Facetten aufgenommen werden. Die Abweichung stieg, je dorsaler die Vorlast angebracht war. Analog stieg auch die minimale Steifigkeit mit zunehmendem dorsalen Abstand der Vorlast zum Widerstandszentrum, während sie bei ventral gelegenen konstant geblieben war. 


\subsection{Singuläre Steifigkeitszunahme als Besonderheit des Segments L3/L4}

Die Versuche von Mansour (2001) zeigten an zwei Präparaten (L3/L4) eine starke singuläre Steifigkeitszunahme bei Platzierung der Vorlast im Wirbelkanal. Die Steifigkeit stieg auf mehr als das Zweifache der dezentralen Messungen. In der vorliegenden Studie konnte zwar ebenfalls eine solche Singularität festgestellt werden, jedoch in weitaus geringerem Maße. Dennoch ist sie eine Eigenschaft, die im angrenzenden L4/L5er-Segment nicht aufgetreten ist (Hawellek 2008). Auf Höhe L1/L2 hingegen konnte Hubert (2009) ebenfalls darstellen, dass die Segmentsteifigkeit bei zentraler Positionierung der Vorlast höher ist als bei ventraler oder dorsaler Lage. Weitaus interessanter ist hingegen der Steifigkeitsabfall bei gering dorsalen Vorlasten. Funktionell bedeutet dies, dass eine winzige Verkippung der beiden Wirbel zueinander ausreicht, um das System von einem stabilen in einen deutlich labileren Zustand zu überführen. Da dieser Effekt am Übergang von der Neutral- zu einer Beinahe-Neutralstellung, also einer häufigen Haltung, auftritt, ist er für den Körper von permanenter Relevanz. Der kurze Moment der Instabilität während der Bewegung muss im richtigen Moment von der Muskulatur aufgefangen werden, ohne den Bewegungsfluss im folgenden stabilen Zustand zu bremsen. Es stellt sich die Frage, warum diese Effekte nur auf dieser Segmenthöhe aufgetreten sind. Möglicherweise hängt es damit zusammen, dass die Ausrichtung dieses Segments während der Messungen ungefähr mit der In-Vivo-Lage übereinstimmt. Die Vorderkanten der Wirbelkörper stehen ungefähr senkrecht im Raum. Allerdings gibt es doch individuelle Unterschiede, die eine gewisse Varianz der Lage zulassen. Weiterhin müsste es dann auf anderen Segmenthöhen möglich sein, durch Anpassung der Versuchsparameter ähnliche Ergebnisse zu erzielen. Entsprechende Daten wurden bisher nicht beschrieben, so dass davon ausgegangen werden kann, dass dieses Phänomen nicht (ausschließlich) durch die räumliche Ausrichtung des Segments zu erklären ist. Ein weiterer Ansatz ergibt sich aus der Orientierung der Facetten. In dieser Studie schwankte der von den beiden Gelenkflächen gebildete Winkel zwischen $50^{\circ}$ und $100^{\circ}$. Winkel von ca. $50^{\circ}$ sind laut Literatur (White und Panjabi 1990) typischerweise auf Höhe L1/L2 zu finden, während das Segment L4/L5 Winkel um $100^{\circ}$ aufweise. Es zeigte sich jedoch keine Korrelation zwischen Größe des Facettenwinkels und Ähnlichkeit unserer Ergebnisse zu denen angrenzender Segmente. Weiterhin fielen innerhalb der vorliegenden Studie die Messdaten trotz der differierenden Gelenkflächenwinkel homogen aus. Somit übernimmt die Ausrichtung der Facetten in der Horizontalen anscheinend keine führende Rolle im Hinblick auf die Bewegungsstruktur. Es ist jedoch möglich, dass makroskopisch nicht sichtbare Unebenheiten oder Knorpelwälle auf den Gelenkflächen für die spezifische Kinematik des L3/L4er-Segments verantwortlich sind. Diese These wurde in dieser Studie nicht untersucht und bedarf weiterer Forschung. 


\subsection{Fazit}

Die Ergebnisse der Versuche bestätigen bzw. legen verschiedene Theorien zur Kinematik der Wirbelsäule nahe. Hierzu gehören:

- Es besteht eine Führung der Bewegung durch eines oder beide Zwischenwirbelgelenke in Abhängigkeit von der Flexions-Extensionsstellung.

- Die Gelenkführung bedingt eine reproduzierbare Wanderung der momentanen Schraubachsen.

- Die Ausrichtung dieser Schraubachsen wird maßgeblich durch die Bandscheibeneigenschaften und den Bandapparat determiniert. Bei Lateralflexion spielen zusätzlich die Gelenke und der Kopplungszustand eine führende Rolle.

- Die Steifigkeit und somit auch der ROM werden von der Ausrichtung und Position der momentanen Schraubachsen, und somit ebenfalls vom Gelenkschluss beeinflusst.

- Die Besonderheit des Segments L3/L4 besteht bei Axialrotation in einem charakteristischen Steifigkeitsmuster bei Kompression im Wirbelkanal.

\subsection{Methodendiskussion}

Der nachfolgende Abschnitt befasst sich mit der kritischen Auseinandersetzung mit der Wahl der Methoden und der Durchführung der Studie. Er beinhaltet die Diskussion der Wirbelsäulenpräparate, der wirksamen Kräfte sowie des Messsystems.

\subsubsection{Präparate}

Zur besten Beurteilung der Biomechanik des Lendenwirbelsegments wäre es wünschenswert, mit optimalem Versuchsgut zu arbeiten. Das heißt, die Präparate sollten frei von jeglichen pathologischen Veränderungen sein. Den qualitativen Ansprüchen werden fast ausnahmslos Präparate junger Erwachsener gerecht. Diese sind jedoch nicht in ausreichender Quantität zu erhalten. Daher wurde in dieser Versuchsreihe auf Spenden älterer Personen zurückgegriffen. Um dennoch ein höchstmögliches Maß an Qualität zu sichern, wurde jedes Präparat vor den Experimenten projektionsradiographisch und computertomographisch untersucht. Bildmorphologisch erfassbare Degeneration oder Traumafolgen galten als Ausschlusskriterium für die Studie. Nach jedem Resektionsschritt erfolgte ebenfalls eine Röntgenaufnahme. Diese Maßnahme dient dem Ausschluss von Läsionen, die durch die Resektionen am Präparat entstanden sein könnten. Die resezierten Gelenke wurden zudem im Anschluss an die Experimente makroskopisch untersucht.

Auch bei der Konservierung der Präparate gilt der Anspruch, die Materialeigenschaften knöcherner und bandhafter Strukturen nicht zu verändern. So wird beispielsweise die Segmentsteifigkeit durch die Zusammensetzung der Fixationslösung beeinflusst (Wilke 
et al. 1996). Daher wurde für diese Experimente die Verwendung einer möglichst schonenden Fixationslösung nach Fanghänel und Schultz (1962) gewählt. Die Relevanz des Erhalts der Bandeigenschaften ist umso wichtiger, da mit dieser Arbeit belegt werden konnte, dass die Bewegungsstruktur des Segments L3/L4 nicht nur von der Morphologie der knöchernen Gelenke abhängt, wie bisher angenommen wurde (Nägerl et al. 1992, Nägerl et al. 1995, Kalscheuer 2001). Vielmehr spielen bei der Ausrichtung der momentanen Schraubachsen vermutlich auch die elastischen Elemente der Wirbelsäule - abhängig von der Bewegungsrichtung - eine Rolle.

\subsubsection{Wahl der wirksamen Kräfte}

Die Möglichkeiten des Körpers, auf die Bewegungsstruktur der Wirbelsäule Einfluss zu nehmen, sind äußerst vielfältig und komplex und dadurch schwer zu messen. In vivo werden Bewegungen selten mit konstanter Geschwindigkeit ausgeführt. Selbst wenn sie über einen längeren Zeitraum gleichmäßig erfolgen, geschieht dies mit lediglich $15 \%$ der Maximalkraft der Muskulatur (Parnianpour et al. 1989). Versuche am lebenden Menschen, bei denen mittels Myographie die Muskelkraft einzelner Muskelgruppen erfasst wird, sind sehr störanfällig (Ralston 1961). Weiterhin ist es für den Probanden schwerlich realisierbar, die Muskelspannung über einen andauernden Zeitraum konstant zu halten. Andererseits führen aber schon geringe Kraftunterschiede bzw. die Anspannung kleinster Muskelgruppen zu einer Variation der Stellung der Wirbel zueinander. Wie bereits diskutiert wurde, sind es gerade diese Stellungsunterschiede, die zu den verschiedenen Kopplungsverhalten der Articulationes zygapophysiales und Spannungszustände der Bandstrukturen führen und somit die Biomechanik determinieren. Daher ist es für diese Studie sinnvoll ein Kraftsystem zu wählen, das gut reproduzierbare und mehrdimensionale Voraussetzungen schafft, die in einzelnen Parametern variiert werden können. Es ist dafür nötig, die komplexen Bewegungsabläufe, die in vivo in der Lendenwirbelsäule entstehen, auf physikalisch messbare zu reduzieren. All dies ist mit der Anbringung der Kraftschraube gelungen. Sie ermöglicht mit ihrer kompressiven Kraftkomponente zusammen mit einem Drehmoment die Simulation der verschiedenen Kraftkonstellationen in sechs Dimensionen. Somit sind alle grundlegenden geometrischen Bewegungsmöglichkeiten in Einem erfasst.

\subsubsection{Axiale Vorlast als kompressive Kraftkomponente}

Die kompressive Komponente der Kraftschraube simuliert das Gewicht des Oberkörpers, das auf den Lendenwirbeln lastet. Dabei entspricht die in der Studie gewählte Vorlast von $200 \mathrm{~N}$ nicht dem reellen Wert, der bekanntermaßen interpersonell sehr variabel ist. Vielmehr ist sie das Ergebnis vorangegangener Untersuchungen. So wurde bereits in verschiedenen Studien (Abumi et al. 1990, Ahmed et al. 1990, Mansour 2001) erfolgreich mit diesem Wert getestet. Andere Arbeiten (Schmidt et al. 2007, Zhu et al. 2007), die sich mit der Belastbarkeit der Segmente und deren Grenzen beschäftigt haben, verwendeten mitunter weit größere kompressive Komponenten (500600 N). In der Zusammenschau zeigte sich, dass die Wahl größerer Kräfte die Mess- 
ergebnisse nicht wesentlich verändert, dafür aber das Risiko steigt, die Präparate während den Messungen zu beschädigen. Somit ist die Wahl einer Vorlast von $200 \mathrm{~N}$, wie in dieser Studie geschehen, begründbar.

Eine weitere Einflussnahme auf die Messergebnisse ist durch den Aufbau der Messapparatur möglich. Cripton et al. (2000) konnten darstellen, dass die Art, wie eine axiale Vorlast appliziert wird, für die Beeinflussung der Messungen von Bedeutung ist. So birgt z.B. die Anbringung eines Druckstempels die Gefahr der Führung der Bewegung, während eine frei hängende Vorlast die Bildung von ungewollten Scherkräften verhindert. In diesem Sinne wurde für die Experimente der vorliegenden Arbeit eine Kraftübertragung über Hebelarme mit Gegenkräften zum Ausgleich des Eigengewichts des Kraftkreuzes gewählt. Diese Methode verhindert zuverlässig die Beeinflussung des Rotationsverlaufs durch das Messsystem selbst und ist ein Verfahren, das einfach anzuwenden und instand zu halten ist. Weiterhin erlaubt die Anbringung der Vorlast über verschiebliche Reiter eine exakte Positionierung der Last mit stets axialer Kraftwirkungslinie.

Möglicherweise könnte die feste Installation des vierten Lendenwirbels an der Messapparatur geringfügige Veränderungen der Messergebnisse verursachen, da die Ausrichtung dieses Wirbels in vivo in Abhängigkeit von der Körperhaltung im Raum variabel ist. Dies hat zur Folge, dass die axiale Kraft in vitro ausschließlich parallel zur z-Achse, in vivo jedoch auch windschief einwirken kann. Zur Klärung der Fragestellungen der vorliegenden Arbeit ist dieser Aspekt jedoch zu vernachlässigen, da Feinheiten dieser Art keine grundsätzlichen Veränderungen in der Segmentkinematik erwarten lassen.

\subsubsection{Drehmoment}

Besonders das Drehmoment impliziert eine potentielle Schädigung des untersuchten Segments durch übermäßige Kraftaufnahme. Um dem vorzubeugen, ist es wichtig, das Präparat nicht über das natürliche Maß hinaus zu beanspruchen. Wilke konnte 1993 graphisch in der Last-Deformation-Kurve darstellen, ab welchem Rotationsbereich eine irreversible Schädigung des Segments zu erwarten ist. Das Prinzip konnte auf die in dieser Arbeit verwendete Drehmoment-Drehwinkel-Kennlinie übertragen werden. Demnach ist eine physiologische Belastung erreicht, sobald der Kurvenverlauf Sigmoidität zeigt. Die Größe des Drehmoments kann dadurch für jedes Präparat individuell ermittelt und an den jeweiligen Resektionszustand angepasst werden. Die Ergebnisse spiegeln demnach das Bewegungsausmaß wider, in dem das Segment sämtliche physiologische Mechanismen der Kinematik zeigt, jedoch nicht durch Überlastung geschädigt werden kann.

\subsubsection{Messsystem}

Zur differenzierten Analyse der Bewegungsstruktur eines Wirbelsäulensegments bedarf es mehr, als lediglich der Ermittlung des Bewegungsausmaßes des untersuchten Präparats. Zu diesem Zweck wurde in dieser Studie ein Verfahren verwendet, das es ermöglicht, die Gesamtbewegung in differentiell kleinen Winkeln zu erfassen. Dieses 
Verfahren erlaubt neben der Ermittlung des Bewegungsausmaßes und der Steifigkeit der gesamten Bewegung die Darstellung der momentanen Schraubachsen in ebendiesen differentiell kleinen Bewegungsabschnitten. Dadurch lässt sich nachweisen, dass die Achsen im Laufe der Rotation nicht nur ihre Position verändern und „wandern“, sondern auch, dass ihre Lage im Raum, also die Ausrichtung variabel ist. Der Detailreichtum dieser Arbeitsweise ist jedoch möglicherweise störanfällig. Fehler, die in der Durchführung entstehen, können sich dabei im Rahmen der elektronischen Verarbeitung der Daten fortführen, so dass ein systematischer Messfehler möglich werden könnte. Besonderes Augenmerk liegt in diesem Zusammenhang auf dem ungenauen Anbringen der Lagesensoren an der fest installierten Messapparatur (Schäfer 1995), ebenso auf fertigungsbedingten Toleranzräumen. Um diesen Fehler möglichst gering zu halten, wurden in dieser Versuchsreihe Testungen mit Objekten durchgeführt (Präzisionsschraube, Bandscheibenmodell), deren Abmessungen und physikalische Parameter bekannt waren. So ließen sich die Einstellungen an der Messapparatur vor Beginn der Versuche kontrollieren und anpassen, um eine hohe Reliabilität und Validität der Ergebnisse zu gewährleisten.

\subsection{Ausblick}

Die Ergebnisse dieser Studie liefern eine Vielzahl von Erkenntnissen über die Bewegungsstruktur des Segments L3/L4. Insbesondere wurde die Funktion der Facettengelenke in den verschiedenen Bewegungsrichtungen in Bezug auf die Position bzw. Wanderung und Ausrichtung der momentanen Schraubachsen verdeutlicht. Weiterhin konnte eine mögliche Beeinflussung der Segmentsteifigkeit und somit auch des Bewegungsausmaßes durch die Facettengelenke dargestellt werden. Die untersuchte Segmenthöhe weist verschiedene anatomische Besonderheiten auf, die sie von anderen Segmenten unterscheidet. Zum einen liegt die Bandscheibe im physiologischen aufrechten Stand des Menschen ungefähr horizontal im Raum, zum anderen gilt der Wirbel L3 als letzter "freier" Wirbel, während L4 bereits ligamentär mit dem Becken verbunden ist. Außerdem variiert die Ausrichtung der Gelenkfacetten im Verlauf der Wirbelsäule. Es ist allerdings zu beachten, dass, wie in dieser Studie gezeigt wurde, die Ausrichtung der Facetten auch innerhalb der Segmente L3/L4 variabel war. So wie dieses Segment, weist jedes Segment der Wirbelsäule pathognomonische Charakteristika auf. Aus diesem Grund ist der dezidierte Vergleich der Kinematik einzelner Segmenthöhen, wie es die Arbeitsgruppe um Wachowski und Nägerl durchführt, zur weiteren Analyse unerlässlich. Zusätzlich sollten zukünftig kinematische Messungen multisegmental, z.B. über zwei Segmente, aber auch über die gesamte Lendenwirbelsäule durchgeführt werden, um neuen Aufschluss über die komplexe Kinematik der Wirbelsäule und die gegenseitige Beeinflussung der Segmentkinematik zu erhalten und im Anschluss auf den lebenden Menschen zu übertragen. Zur Umsetzung solcher Messungen ist jedoch die Weiterentwicklung der vorhandenen Messapparatur nötig. Beispielsweise wäre dann ein größerer Bewegungsumfang der Messobjekte zu erwarten, der eventuell eine Umstellung auf andere Verfahren der Bewegungsmessung, z.B. mit 
optischen Markern, notwendig macht. Eine Weiterentwicklung der Messapparatur wäre auch im Hinblick auf die Fehlerberechnung und -minimierung wünschenswert. Zu diesem Zweck könnte die Anzahl der Messtaster auf neun oder zwölf erhöht werden. Dadurch würde es möglich, die einzelnen Ebenen des Koordinatensystems unabhängig voneinander zu bestimmen. Eine weitere mögliche Fehlerquelle könnte in einer Einflussnahme auf die Segmenteigenschaften durch die Fixierlösung bestehen. Um diese Möglichkeit eindeutig auszuschließen, könnte ein Vergleich von Messungen an frischem und mittels Fixierlösung konserviertem Material stattfinden. Für diese Zwecke wäre der Einsatz tierischer Segmente denkbar. Die aus diesen Versuchen gewonnenen Ergebnisse könnten im Anschluss dem Vergleich der humanen und tierischen Segmentkinematik dienen.

In der vorliegenden Studie sind neben Gemeinsamkeiten auch einige individuelle Unterschiede zwischen den einzelnen Präparaten auffällig geworden. Demnach wäre es von großem wissenschaftlichen Interesse, in Folgearbeiten eine größere Anzahl an Präparaten zu testen und diese vorab nach Merkmalen zu gruppieren. So könnten beispielsweise mögliche Einflüsse des Geschlechts oder des Alters der Probanden auf die Kinematik ermittelt werden. Eine weitere Gruppierung wäre nach der Ausrichtung der Facettengelenke möglich, und zwar einerseits nach der Größe des Facettenwinkels, andererseits nach der vertikalen Ausrichtung der Facetten. Durch den Vergleich der Gruppen wäre anschließend der Rückschluss auf weitere Einflussfaktoren der Segmentkinematik möglich.

Ein großes Forschungsfeld bietet im Zusammenhang mit biomechanischen Untersuchungen der Wirbelsäule die kinematische Aufarbeitung morphologischer Pathologien. Da die vorliegende Arbeit sich detailliert mit der Bewegungsstruktur der gesunden Wirbelsäule beschäftigt, wäre der Vergleich der hier ermittelten Befunde mit den kinematischen Mustern der häufigsten Wirbelsäulenpathologien (z.B. Arthrose, Bandscheibenveränderungen) relevant. Aus den daraus ermittelten Ergebnissen ließen sich im Anschluss neue Therapie- und Präventionsansätze finden und Grundprinzipien etablieren. Beispielsweise könnten, ähnlich wie bei der Ganganalyse, pathologische Bewegungsmuster erkannt und physiotherapeutisch neue Muster erlernt werden. Voraussetzung hierfür wäre die genaue Kenntnis der physiologischen Wirbelsäulenkinematik, wie sie Thema dieser Arbeit ist. Weiterhin könnten sich aus dem Vergleich Hinweise ergeben auf biomechanische Ursachen von (chronischen) Rückenschmerzen von Patienten, bei denen mit der bisherigen Diagnostik keine Pathologien festgestellt werden konnten.

Letztendlich kann die Erforschung der oben genannten Punkte neben der Verbesserung präventiver und konservativer Ansätze auch neue bzw. modifizierte operative Verfahren der Behandlung von Rückenschmerzen ergeben. Eine gängige Methode solcher operativer Verfahren besteht nach wie vor in der Implantation von Bandscheibenprothesen, die als „bewegungserhaltende“ Maßnahme den natürlichen Zustand imitieren sollen. Bisher war jedoch nur wenig darüber bekannt, inwiefern die Kinematik der Segmente dadurch verändert und gestört wird. Die Vielzahl der Funktionsprinzipien der eingesetzten Implantate weist zusätzlich darauf hin, dass bislang keine zufriedenstel- 
lende Imitation der physiologischen Bewegungsstruktur erreicht werden konnte. Auf der Basis der mit dieser Studie erhobenen Befunde kann in Zukunft ein Vergleich mit mit Prothesen versehenen Wirbelsäulensegmenten stattfinden, um auf diese Weise die Funktion der Prothesen zu optimieren. Erste Messungen wurden bereits in der Arbeitsgruppe um Wachowski im Bereich der Hals- und Lendenwirbelsäule durchgeführt. Die Versuche könnten zukünftig sowohl auf weitere Segmenthöhen als auch auf andere Modelle von Implantaten ausgeweitet werden. Dieser Bereich bedarf also vertiefender Erforschung. Im Verlauf wären Langzeitstudien zum Erfolg - darstellbar durch Daten zu Schmerzreduktion, Beweglichkeit und Lebensdauer - dieses Therapieverfahrens wünschenswert. 


\section{Zusammenfassung}

Das Funktionsprinzip der Wirbelsäule basiert auf einem komplexen System, das sich nicht allein durch das Wissen über die anatomischen Verhältnisse erklären lässt. Vielmehr ist die Physiologie und Biomechanik der einzelnen Wirbelsäulensegmente von übergeordneter Bedeutung. Bislang ist die Datenlage zu den Teilbereichen jedoch unzureichend. Dies ist zum einen durch eine mangelhafte Auflösung und Präzision der in den bisherigen Studien verwendeten Versuchsapparaturen begründet. Zum anderen schließen die bis heute durchgeführten Studien nur einen Teil der möglichen Bewegungen der Wirbelsäule ein, so dass kein umfassendes Bild über die Biomechanik der Lendenwirbelsäule erstellt werden kann. Vorangegangene Versuchsreihen weisen auf eine Sonderstellung des Segments L3/L4 hin. So wurde ein ungewöhnliches Steifigkeitsverhalten dieses Segments unter bestimmten Versuchsbedingungen festgestellt, das detaillierter Untersuchungen bedarf.

Aus diesen Gründen wurde in der vorliegenden Studie eine Messapparatur gewählt, die eine sechs-dimensionale Aufzeichnung differentiell kleiner Bewegungsabschnitte registriert. Die Datenerhebung erfolgte an humanen Wirbelsäulenpräparaten (L3/L4), an denen pathologische Veränderungen ausgeschlossen wurden. Die Präparate wurden dem variablen Kräftepaar axiale Kraft (Vorlast) und Drehmoment ausgesetzt, um verschiedene Körperhaltungen und -bewegungen zu simulieren. Die resultierende Positionsänderung von L3 konnte kontinuierlich aufgezeichnet und aus den Werten die Messparameter zur detaillierten Bewegungsanalyse ermittelt werden. Zur Klärung der Rolle der Wirbelbogengelenke wurden die Messungen am intakten Segment sowie nach konsekutiver Resektion der Wirbelbogengelenke durchgeführt. Auf diese Weise konnte durch Vergleich der Messergebnisse ein Rückschluss auf die Funktion der Gelenke durchgeführt werden.

Die Ergebnisse der Messungen verdeutlichten eine Abhängigkeit der Position und des Wanderungsverlaufs der momentanen Schraubachsen sowohl von der Vorlastposition als auch vom Resektionsstatus. Im Gegensatz dazu war die Ausrichtung der Achsen hauptsächlich durch die Vorlastposition und weniger durch die Resektion der Gelenke zu beeinflussen. Lediglich in der Lateralflexion wurde auch die Achsausrichtung maßgeblich vom Resektionsstatus bestimmt. Die Segmentsteifigkeit zeigte sich ebenfalls gebunden an die Positionierung der Vorlast. Es wurden unter Axialrotation jedoch Abweichungen von einem linearen Verhalten sichtbar, wenn die Vorlast um den Wirbelkanal herum angebracht war. Dieser Effekt konnte besonders deutlich am intakten Segment dargestellt werden. Das Bewegungsausmaß verhielt sich stets reziprok zur Segmentsteifigkeit.

Die Analyse der Ergebnisse dieser Studie legt die Führung der Bewegung durch die Zwischenwirbelgelenke nahe. Diese Führung wird wahrscheinlich durch Kraftschlüssigkeit der Gelenkfacetten ermöglicht, die durch Extension des Segments oder rotati- 
onsbedingten Kontakt zwischen den Facetten hervorgerufen wird. In Abhängigkeit von der Flexions-Extensionsstellung und des Drehmoments liegt das Führungsverhalten entweder als Ein- oder Zwei-Gelenk-Führung vor. Bei starker Flexion ist zudem ein Zustand ohne Gelenkführung möglich, wenn beide Wirbelbogengelenke entkoppelt sind. Die Funktion der Bandstrukturen liegt vermutlich hauptsächlich in der Stabilisierung des Segments. Lediglich bei resezierten Segmenten könnten sie die Bewegungsführung übernehmen. Die Steifigkeit und somit auch das Bewegungsausmaß werden von der Ausrichtung und Position der momentanen Schraubachsen und somit ebenfalls vom Gelenkschluss beeinflusst.

Die besondere Stellung des Segments L3/L4 manifestierte sich nicht, wie in vorherigen Untersuchungen angedeutet wurde, in einer besonders hohen Steifigkeit bei axialer Last im Wirbelkanal, sondern in einem Absinken bei gering dorsal gelegenen Vorlasten. Dies bedeutet für den beschriebenen Moment einen Stabilitätsverlust der Wirbelsäule. Die Ursache konnte mit der vorliegenden Studie nicht eindeutig geklärt werden. Möglicherweise ist sie auf makroskopische Unebenheiten der Gelenkflächen zurückzuführen. Dieser These kann in weiterführenden Untersuchungen ebenso nachgegangen werden wie den Veränderungen in der Biomechanik, die mit den gängigen Wirbelsäulenerkrankungen einhergehen. Mit den Ergebnissen der Studien zu pathologischen Bewegungsstrukturen können, da ein Vergleich mit den Daten der vorliegenden Arbeit möglich ist, sowohl etablierte Therapieverfahren weiterentwickelt als auch neue Konzepte zur Prävention dieser Erkrankungen erstellt werden. 


\section{Anhang A: Fehlerabschätzung bei der Erzeugung des Drehmoments}

Bei dem in dieser Studie verwendeten Seilzug-Prinzip kommt es durch die Rotation des Drehkreuzes zu einer Abweichung von der lotrechten Seilführung, die zur Entstehung einer Rückstellkraft führt, die dem Drehmoment entgegenwirkt. Die Abbildung 69 zeigt exemplarisch die Auswirkungen, die durch eine solche Abweichung zustande kommen. b sei die konstante Länge des Armes des Drehkreuzes, I die Länge des Seils bis zu der Umlenkrolle in Neutralstellung. Bei einer Rotation des Armes um den Winkel a vergrößert sich I näherungsweise um die Länge c. Durch die resultierende Richtungsänderung der konstanten Kraft $F_{r}$ wirkt nun ${\underline{F_{r}}}_{r}$. Für eine vektorielle Berechnung von $\underline{F}_{r}^{*}$ aus $\underline{F}_{r}$ benötigt man den Kraftvektor $\underline{G}$, der der Rückstellkraft entspricht. Es folgt:

$\underline{\mathrm{F}}_{\underline{r}}^{*}=\underline{\mathrm{F}_{\mathrm{r}}}+\underline{\mathrm{G}}$.

Aus den trigonomischen Zusammenhängen ergibt sich:

$$
\begin{aligned}
& a=2 b \cdot \sin (\alpha / 2)=d \cdot \sin (-1 \alpha / 2) \\
& \Rightarrow \quad \mathrm{d}=2 \mathrm{~b} \cdot \sin (2 \mathrm{\alpha} / 2) \\
& \text { und: } \quad \tan (\alpha / 2) \mathrm{cd}^{-1}=\sin (\alpha / 2) \cdot \cos (-1 \alpha / 2) \quad \text { (II) } \\
& \text { (I), (II) } \Rightarrow \quad \text { C }=2 b \cdot \sin (3 \alpha / 2) \cdot \cos (-1 \alpha / 2) \quad \text { (III) } \\
& \text { Sowie: } \quad \beta=\arctan \left[\mathrm{d} \cdot(\mathrm{I}-\mathrm{c})^{-1}\right] \quad \text { (IV) } \\
& \text { (I), (II), (IV) } \Rightarrow \quad \beta=\arctan \left[2 b \cdot \sin (2 \alpha / 2) \cdot(I-2 b \cdot \sin (3 \alpha / 2) \cdot \cos (-1 \alpha / 2))^{-1}\right] \\
& \text { Im gleichschenkligen Dreieck }\left(\left|\underline{\underline{F}}_{r}^{*}\right|=\left|\underline{\underline{F}}_{\underline{r}}\right|\right) \\
& \begin{aligned}
\sin (\beta / 2) & =1 / 2 G \cdot F_{r}^{-1} \\
G & =2 F_{r} \cdot \sin (\beta / 2)
\end{aligned}
\end{aligned}
$$

Mit $b=15 \mathrm{~cm}, \mathrm{I}=50 \mathrm{~cm}$ und $\alpha_{\max }=4^{\circ}$ ergeben sich für $\beta_{\max }=0,04^{\circ}$ und für $\mathrm{G}_{\max }=7,3 \cdot 10^{-4}$.

Für $F_{r \text { max }}=9,81 \mathrm{~N}$ erhält man $G_{\max }=7,17 \cdot 10^{-3} \mathrm{~N}$. Damit ist die Rückstellkraft vernachlässigbar klein. 


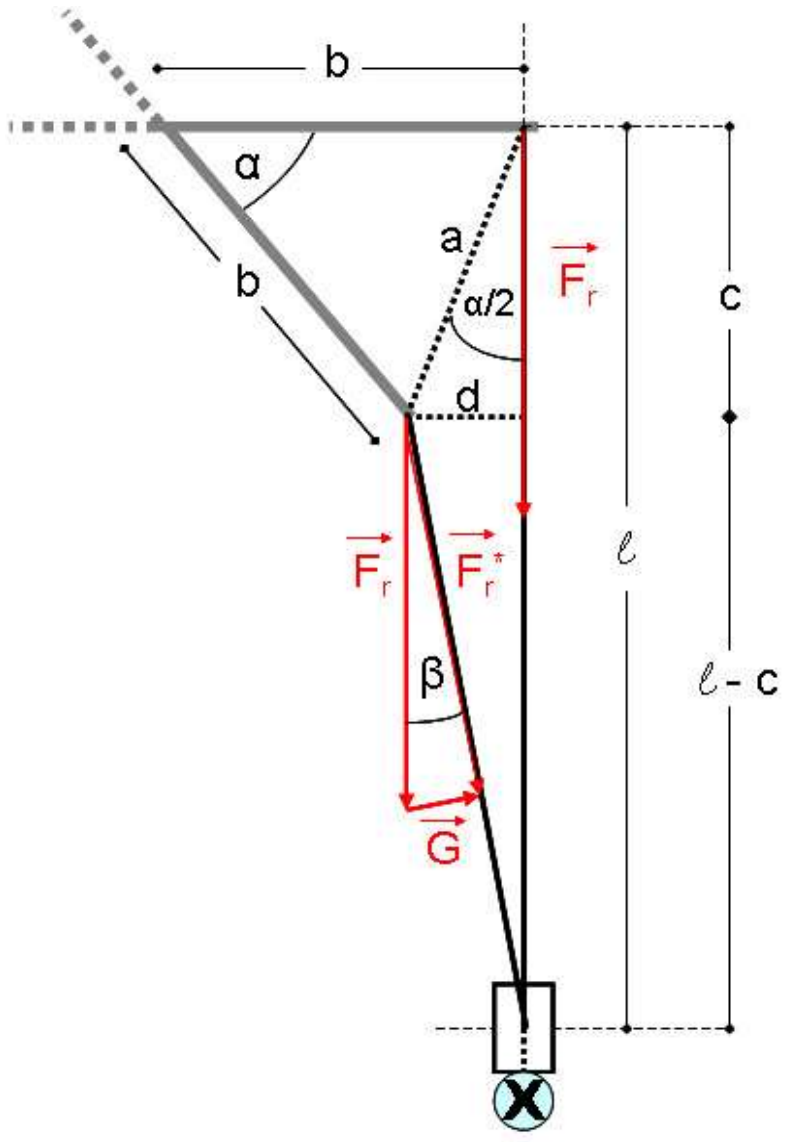

Abbildung 69: Fehlerabschätzung bei der Erzeugung des Drehmoments (Mansour 2001, S. 66) 


\section{Anhang B: Bestimmung der Positionsänderung}

Man kann die Bewegung eines Objekts als Wanderung von dem Ursprungskoordinatensystem $\mathrm{K}_{0}$ in ein gewandertes Koordinatensystem $\mathrm{K}_{n}$ beschreiben. In diesem Fall definiert der untere Wirbel $\mathrm{K}_{0}$, während der obere Wirbel $\mathrm{K}_{n}$ darstellt. In der Ausgangssituation (Zeitpunkt $t_{0}$ ) entspricht $K_{0}$ der Lage von $K_{n}$, die Definition der Ebenen erfolgt durch die Nullstellung der sechs Messtaster. Sobald es durch das Anlegen des Kraftsystems zu einer Verkippung des oberen Wirbels kommt, ändern sich die Tasterwerte und $\mathrm{K}_{0}$ ist von $\mathrm{K}_{\mathrm{n}}$ verschieden.

$\mathrm{K}_{0}$ lässt sich folgendermaßen beschreiben:

Die $x-y$-Ebene wird mit den Werten der drei Messtaster $T_{1}, T_{2}$ und $T_{3}$ über die Vektoren

$\underline{\mathrm{V}}_{1}=\underline{\mathrm{T}}_{1}-\underline{\mathrm{T}}_{2}$ und

$\underline{\mathrm{V}}_{2}=\underline{\mathrm{T}}_{1}-\underline{\mathrm{T}}_{3} \quad$ festgelegt.

Der Richtungsvektor $\underline{\mathrm{N}}_{z 0}$ der z-Achse lässt sich aus dem Kreuzprodukt von $\underline{\mathrm{V}}_{1}$ und $\underline{\mathrm{V}}_{2}$ berechnen:

$\underline{N}_{z 0}=\underline{V}_{1} \times \underline{V}_{2} \leftrightarrow \underline{N}_{z 0}=\left(\underline{T}_{1}-\underline{T}_{2}\right) \times\left(\underline{T}_{1}-\underline{T}_{2}\right)$

Die $y$-z-Ebene ergibt sich durch Orthogonalität der Ebenen aus den Tastern $T_{4}$ und $T_{5}$ durch die Vektoren

$\underline{\mathrm{V}}_{3}=\underline{\mathrm{V}}_{1} \times \underline{\mathrm{V}}_{2}\left(\underline{\mathrm{V}}_{3} \perp \underline{\mathrm{V}}_{1} \wedge \underline{\mathrm{V}}_{3} \perp \underline{\mathrm{V}}_{2}\right)$

$\underline{\mathrm{V}}_{4}=\underline{\mathrm{T}}_{4}-\underline{\mathrm{T}}_{5}$

und dem Richtungsvektor $\underline{N}_{x 0}$ der $x$-Achse: $\underline{N}_{x 0}=\underline{N}_{z 0} \times \underline{V}_{4}$.

Zur Bestimmung der der x-z-Ebene benötigt man zusätzlich den Taster $\mathrm{T}_{6}$. Es gilt:

$\underline{\mathrm{V}}_{5}=\underline{\mathrm{V}}_{3} \times \underline{\mathrm{V}}_{4}$ und

$\underline{\mathrm{V}}_{6}=\left(0, \underline{\mathrm{T}}_{6}, 0\right)$.

Richtungsvektor der y-Achse: $\underline{\mathrm{N}}_{\mathrm{Y} 0}=\underline{\mathrm{N}}_{\mathrm{X0}} \times \underline{\mathrm{N}}_{\mathrm{ZO}}$.

Analog kann bei Positionsänderungen das Koordinatensystem $K_{n}$ zum Zeitpunkt $t_{n}$ mithilfe der Tasterwerte $T_{1 n}-T_{6 n}$ berechnet werden.

Um die Verschiebungen des Koordinatensystems zwischen den Zeitpunkten $t_{0}, t_{n}$ und $t_{n-1}$ darzustellen, wird der Translationsvektor $\underline{d}_{0}$ durch Bildung der Differenzvektoren der Koordinatenursprünge bestimmt:

$\underline{\mathrm{d}}=\underline{\mathrm{k}}_{\mathrm{n}}-\underline{\mathrm{k}}_{\mathrm{n}-1}$

Da nun die Translationskomponente der Bewegung zu errechnen ist, wird auch die Rotationskomponente benötigt, um die komplette Bewegung darzustellen. 
Der Rotationsvektor $\underline{\mu}_{n}\left(\underline{\mu}_{x}, \underline{\mu}_{y}, \underline{\mu}_{z}\right)$ beschreibt die Rotation des Koordinatensystems $K_{n}$ relativ zu dem Ursprungskoordinatensystem $\mathrm{K}_{0}$.

$\tan \mu_{\mathrm{xn}}=\left(\Delta \mathrm{z}_{\mathrm{c}}-\Delta \mathrm{z}_{\mathrm{a}}\right) /\left(\mathrm{y}_{\mathrm{c}}-\mathrm{y}_{\mathrm{a}}\right)$

$\tan \mu_{\mathrm{yn}}=\left(\Delta \mathrm{z}_{\mathrm{b}}-\Delta \mathrm{z}_{\mathrm{a}}\right) /\left(\mathrm{y}_{\mathrm{a}}-\mathrm{y}_{\mathrm{b}}\right)$

$\tan \mu_{z n}=\left(\Delta z_{e}-\Delta z_{d}\right) /\left(y_{d}-y_{e}\right)$

Die Definition der Vorzeichen der Werte erfolgt im mathematischen positiven Sinne im Rechtshändigen Koordinatensystem. Eine Drehmatrix entfällt wenn $\sin \alpha \cong \alpha \wedge \cos \alpha \cong \alpha$. 


\section{Anhang C: Berechnung der Schraubparameter}

Um die Bewegung des oberen Wirbels relativ zum unteren zu beschreiben, wird das „Konzept der wandernden Schraubachsen“ von Goldstein (1983) verwendet. Es besagt, dass die Bewegung eines Objekts, also die Lageveränderung des Koordinatensystems $K_{n}$ gegen $K_{n-1}$ in differentiell kleine Schritte zerlegt werden kann, wobei jeder dieser Schritte einer Schraubung um eine momentane Schraubachse $d_{n}(\alpha)$ entspricht. Zur Definition einer Schraubachse genügt ihre Richtung und das Ausmaß der Rotation um sie herum. Der Richtungsvektor ergibt sich aus dem Rotationsvektor $\mu$ und dem Einheitsvektor $\underline{\mathrm{e}}$.

$\underline{e}=1 /|\underline{\mu}| \cdot\left(\mu_{x}, \mu_{y}, \mu_{z}\right)$.

Dabei entspricht der Betrag des Rotationsvektors $|\underline{\mu}|$ dem Rotationswinkel $\mu$ um die Schraubachse. Die Schraubsteigung T ergibt sich wie folgt:

$\underline{I}=\mathrm{s} / \mu=\underline{\mathrm{d}} \cdot \underline{\mu} /|\mu|^{2}$

$\mathrm{s}$ ist der Versatz entlang der Schraubachse und wird gebildet, indem man den Translationsvektor $\underline{\mathrm{d}}$ auf die Achse bzw. ihren normierten Richtungsvektor $\underline{e}$ projiziert.

$\mathrm{S}=\underline{\mathrm{d}} \cdot \underline{\mathrm{e}}$

Der kürzeste Abstand a der Schraubachsengeraden zum Koordinatenursprung, also der Ortsvektor a der Geraden, ist der Aufpunktvektor. Er errechnet sich nach Teichmann (1973) mit

$\underline{a}=0,5 \cdot[\underline{d}-s \cdot \underline{e}+\cot (\mu / 2) \cdot(\underline{e} \times \underline{d})]$.

Durch die Bestimmung der Schraubachsen $r_{i}$ in Ausrichtung auf das Ursprungskoordinatensystem $\mathrm{K}_{0}$ kann der absolute Rotationswinkel $\alpha$ des oberen Wirbels ermittelt werden. Bei einem zusätzlichen Bezug auf das bewegte Koordinatensystem $K_{n-1}$ kann auBerdem der differentielle Drehwinkel $d \mu$, der Versatz ds und die Schraubsteigung $d T$ ermittelt werden. Die Darstellung der Rastpolkurve erfolgt bezogen auf das Ursprungskoordinatensystem.

Fehlerbetrachtung:

Der Drehwinkel $d \mu$ wird direkt aus dem Rotationsvektor $\mu$ abgeleitet, daher ist sein Fehler klein. Anders verhält es sich bei der Bestimmung der differentiellen Schraubachsen. Sie werden mit der Winkeldifferenz $\Delta \mu$ anstelle des Drehwinkels $d \mu$ ermittelt. Somit wird die Messung genauer, je kleiner die Winkel gewählt werden; dies führt jedoch auch zu einem größeren zufälligen, relativen Messfehler. Da zur Berechnung der Schraubsteigung t (s.o.) das Quadrat des Rotationswinkels $|\mu|$ verlangt wird, setzt sich auch der Fehler quadratisch fort. 


\section{Abbildungsverzeichnis}

Abbildung 1: Knöcherner LWK 4 (Der Abdruck dieser Abbildung erfolgt mit freundlicher Genehmigung des Thieme-Verlags.).

Abbildung 2: Junghans'sches Bewegungssegment (Der Abdruck dieser Abbildung erfolgt mit freundlicher Genehmigung des Thieme-Verlags.)..... 6

Abbildung 3: Schematische Darstellung der Wirbelköperbänder und Wirbelbogenbänder (Der Abdruck dieser Abbildung erfolgt mit freundlicher Genehmigung des Thieme-Verlags.).

Abbildung 4:Vergrößerte Darstellung von zwei benachbarten Wirbelbogengelenken der HWS (Der Abdruck dieser Abbildung erfolgt mit freundlicher Genehmigung des Thieme-Verlags.) und CT-Bild eines Wirbelbogengelenks auf Höhe L3/L4

Abbildung 5: Unbekannte Bewegung eines Körpers von 1 nach 2 (Der Abdruck dieser Abbildung erfolgt mit freundlicher Genehmigung von Dr. Bockermann.)

Abbildung 6: Modell einer Synarthrose

Abbildung 7: Modell einer überschlagenen Gelenkkette

Abbildung 8: Modell einer überschlagenen dimeren Gelenkkette vor und nach einer differentiell kleinen Elementarbewegung....

Abbildung 9: Modell einer symmetrischen Viergelenkkette (Der Abdruck dieser Abbildung erfolgt mit freundlicher Genehmigung des Thieme-Verlags.).... 14

Abbildung 10: Röntgen des eingebetteten Präparats A ........................................ 16

Abbildung 11: Röntgen des eingebetteten Präparats B …...................................... 16

Abbildung 12: Röntgen des eingebetteten Präparats C .................................... 17

Abbildung 13: Röntgen des eingebetteten Präparats D ........................................... 17

Abbildung 14: Röntgen des eingebetteten Präparats E ..................................... 17

Abbildung 15: Transversale Schnittbilder der Präparate A-E mit eingezeichnetem Facettenwinkel $\varphi$................................................................. 18

Abbildung 16: Coronale Schnittbilder der Präparate A-E mit eingezeichnetem Abweichungswinkel $\omega$ der Gelenkfacetten von einer vertikalen Linie........ 19

Abbildung 17: Messstand und Drehkreuz ....................................................... 21

Abbildung 18: Gewählte Positionen zur Anbringung der Vorlast. .............................. 22

Abbildung 19: Prinzip der Anbringung der Vorlast an verschieblichen Reitern ........... 22

Abbildung 20: Skizze der Messapparatur .......................................................... 23

Abbildung 21: Räumliche 3-2-1-Anordnung der sechs Messtaster............................ 24

Abbildung 22: Präzisionsschraube und Bandscheibenmodell mit Gelenkmodellen ... 26

Abbildung 23: Beispiel einer Rastpolkurve unter Axialrotation. ............................. 27

Abbildung 24: Beispiel einer Drehwinkel-Drehmoment-Kennlinie............................ 29

Abbildung 25: Beispiel einer Steifigkeitskurve................................................. 30

Abbildung 26: Rastpolkurven von Präzisionsschraube und Bandscheibenmodell ..... 33

Abbildung 27: Rastpolkurven des Bandscheiben-Gelenk-Modells ........................... 34

Abbildung 28: Rastpolkurven des intakten Präparats bei zentraler, ventraler und dorsaler Vorlast; Axialrotation ........................................................... 36

Abbildung 29: Rastpolkurven nach Resektion des rechten Zwischenwirbelgelenks bei zentraler, ventraler und dorsaler Vorlast; Axialrotation. 
Abbildung 30: Rastpolkurven des Bandscheiben-Bänder-Präparats bei zentraler, ventraler und dorsaler Vorlast; Axialrotation

Abbildung 31: Vergleich der Rastpolkurven bei ventraler Vorlast bei unterschiedlichen Resektionszuständen; Axialrotation.

Abbildung 32: Rastpolkurven des intakten Präparats bei zentraler und rechtslateraler Vorlast; Axialrotation

Abbildung 33: Rastpolkurven nach Resektion des rechten Zwischenwirbelgelenks bei lateralen Vorlasten; Axialrotation.

Abbildung 34: 3-D-Aufzeichnung der Wanderung der IHA bei ventraler und dorsaler Vorlastposition; intaktes Präparat; Axialrotation.

Abbildung 35: Mittlere ventro-dorsale Abweichung der IHA von der z-Achse; Axialrotation.

Abbildung 36: Drehwinkel-Drehmoment-Kennlinie des intakten Präparats sowie des Bandscheiben-Bänder-Präparats; Axialrotation............................... 47

Abbildung 37: Mittlerer ROM bei sagittalen Vorlastpositionen; Axialrotation. ............ 49

Abbildung 38: Mittlerer ROM bei lateralen Vorlastpositionen; Axialrotation................ 50

Abbildung 39: Steifigkeitskurven im intakten Segment und nach Resektion des rechten Zwischenwirbelgelenks; Axialrotation...................................... 51

Abbildung 40: Mittlere minimale Steifigkeit bei sagittalen Vorlastpositionen; Axialrotation.

Abbildung 41: Mittlere relative Steifigkeit bei sagittalen Vorlastpositionen; Axialrotation.

Abbildung 42: Mittlere minimale Steifigkeit bei lateralen Vorlastpositionen; Axialrotation.

Abbildung 43: Rastpolkurven bei unterschiedlichen Größen der Vorlast; FlexionExtension.

Abbildung 44: Rastpolkurven des intakten Präparats bei sagittalen Vorlastpositionen; Flexion-Extension.

Abbildung 45: Rastpolkurven nach Resektion des rechten Wirbelbogengelenks bei sagittalen Vorlastpositionen; Flexion-Extension

Abbildung 46: Rastpolkurven des Bandscheiben-Bänder-Präparats bei sagittalen Vorlastpositionen; Flexion-Extension

Abbildung 47: Rastpolkurven des intakten Präparats bei zentraler und rechtslateraler Vorlastposition; Flexion-Extension

Abbildung 48: Rastpolkurven nach Resektion des rechten Wirbelbogengelenks bei lateralen Vorlastpositionen; Flexion-Extension....

Abbildung 49: Rastpolkurven des Bandscheiben-Bänder-Präparats bei lateralen Vorlastpositionen; Flexion-Extension

Abbildung 50: Drehwinkel-Drehmoment-Kennlinie bei ventraler und dorsaler Vorlast; Flexion-Extension..

Abbildung 51: Mittlerer ROM bei sagittalen Vorlastpositionen; Flexion-Extension..... 67

Abbildung 52: Mittlerer ROM bei lateralen Vorlastpositionen; Flexion-Extension. ..... 68

Abbildung 53: Steifigkeitskurven bei unterschiedlichen Vorlastpositionen; FlexionExtension.

Abbildung 54: Mittlere minimale Steifigkeit bei sagittalen Vorlastpositionen; Flexion-Extension.

Abbildung 55: Mittlere minimale Steifigkeit bei lateralen Vorlastpositionen;

Flexion-Extension.

Abbildung 56: Rastpolkurven des intakten Präparats bei sagittalen

Vorlastpositionen; Lateralflexion. 
Abbildung 57: Rastpolkurven nach Resektion des rechten Wirbelbogengelenks bei sagittalen Vorlastpositionen; Lateralflexion

Abbildung 58: Rastpolkurven des Bandscheiben-Bänder-Präparats bei sagittalen Vorlastpositionen; Lateralflexion.

Abbildung 59: Vergleich der Rastpolkurven bei zentraler Vorlast bei unterschiedlichen Resektionszuständen; Lateralflexion.

Abbildung 60: Vergleich der Rastpolkurven bei dorsaler Vorlast bei unterschiedlichen Resektionszuständen; Lateralflexion

Abbildung 61: Rastpolkurven des intakten Präparats bei lateralen Vorlastpositionen; Lateralflexion

Abbildung 62: Rastpolkurven nach Resektion des rechten Wirbelbogengelenks bei lateralen Vorlastpositionen; Lateralflexion

Abbildung 63: Rastpolkurven des intakten Präparats bei unterschiedlichen Vorlastgrößen; Lateralflexion

Abbildung 64: Rastpolkurven des Bandscheiben-Bänder-Präparats bei unterschiedlichen Vorlastgrößen; Lateralflexion.

Abbildung 65: Mittlerer ROM bei sagittalen Vorlastpositionen; Lateralflexion. 84

Abbildung 66: Mittlerer ROM bei lateralen Vorlastpositionen; Lateralflexion.

Abbildung 67: Mittlere minimale Steifigkeit bei sagittalen Vorlastpositionen; Lateralflexion

Abbildung 68: Mittlere minimale Steifigkeit bei lateralen Vorlastpositionen; Lateralflexion.

Abbildung 69: Fehlerabschätzung bei der Erzeugung des Drehmoments 


\section{Tabellenverzeichnis}

Tabelle 1: Facettenwinkel $\varphi$ aller Präparate ...................................................... 18

Tabelle 2: Abweichung der Ausrichtung der Gelenkfacetten von einer vertikalen Linie in ${ }^{\circ}$

Tabelle 3: Beispieltabelle: Abweichung nach lateral der momentanen Schraubachse.

Tabelle 4: Vergleich der erwarteten und gemessenen Ergebnisse der Messung der Präzisionsschraube

Tabelle 5: Mittelwerte der ventro-dorsalen Abweichung der momentanen

Schraubachse; Axialrotation.

Tabelle 6: Mittelwerte der lateralen Abweichung der momentanen Schraubachse;

Axialrotation.

Tabelle 7: Mittlerer ROM; Axialrotation

Tabelle 8: Mittlere minimale Steifigkeit; Axialrotation ............................................ 53

Tabelle 9: Zusammenfassung der Ergebnisse des Experiments "Axialrotation" ........ 55

Tabelle 10: Mittelwerte der ventro-dorsalen Abweichung der momentanen

Schraubachse; Flexion-Extension.

Tabelle 11: Mittelwerte der kranio-kaudalen Abweichung der momentanen

Schraubachse; Flexion-Extension.

Tabelle 12: Mittlerer ROM; Flexion-Extension...................................................... 67

Tabelle 13: Mittlere minimale Steifigkeit; Flexion-Extension.................................... 70

Tabelle 14: Zusammenfassung der Ergebnisse des Experiments „Flexion-

Extension“

Tabelle 15: Mittelwerte der kranio-kaudalen Abweichung der momentanen Schraubachse; Lateralflexion.

Tabelle 16: Mittelwerte der lateralen Abweichung der momentanen

Schraubachse; Lateralflexion.............................................................. 82

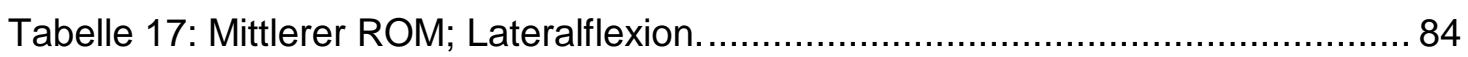

Tabelle 18: Mittlere minimale Steifigkeit; Lateralflexion ...................................... 87

Tabelle 19: Zusammenfassung der Ergebnisse des Experiments „Lateralflexion“ .... 88 


\section{Literaturverzeichnis}

Abumi K, Panjabi MM, Kramer K, Durancem J, Oxland T, Crisco J (1990): Biomechanical evaluation of lumbar spinal stability after graded facetectomies. Spine $\underline{15-11}, 1142-1147$

Adams MA, Hutton WC (1980): The effect of posture on the role of the apophysial joints in resisting intervertebral compressive forces. J Bone Joint Surg $\underline{62-B}$, 358-362

Ahmed AM, Duncan NA, Burg DL (1990): The effect of facetgeometrie on the axial torque rotation response of the lumbar motion segments. Spine $\underline{15}, 391-401$

Bartels RH, Donk RD, Pavlov P, van Limbeek J (2008): Comparison of biomechanical properties of cervical artificial disc prosthesis: a review. Clin Neurol Neurosurg $\underline{110}, 963-967$

Baumgartner WR: Die Bewegung einzelner Lendenwirbel unter Altagsbelastungen. Naturwiss. Diss. Zürich 2000

Benninghoff A, Drenckhahn D: Anatomie. Makroskopische Anatomie, Histologie, Embryologie, Zellbiologie. Band 1. 16. Auflage; Urban und Fischer, München, Jena 2002

Benninghoff A, Drenckhahn D: Anatomie. Makroskopische Anatomie, Histologie, Embryologie, Zellbiologie. Band 1. 17. Auflage; Urban und Fischer, München, Jena 2008

Biederer J, Hutzelmann A, Rama B, Heller M (1999): Röntgen-

Verlaufsuntersuchungen der Halswirbelsäule nach anteriorer Fusion mit Titaninterponaten. MRT Fortschr Röntgenstr 171, 95-99

Bockermann V: Kinematik des Halswirbelsäulen-Segments C5/6. Biomechanische Analyse seines Bewegungsmusters bei physiologischen Standardbelastungen. Med. Diss. Greifswald 2004

Bogduk N, Mercer S (2000): Biomechanics of the cervical spine. I: Normal kinematics. Clin Biomech $\underline{15}, 633-648$

Chow DH, LUK KD, Evans JH, Leong JC (1996): Effects of short anterior lumbar interbody fusion on biomechanics of neighboring unfused segments. Spine 21, 549-555

Clausen JD, Goel VK, Traynelis VC, Scifert J (1997): Uncinate processes and Luschka joints influence the biomechanics of the cervical spine: Quantification using a finite element model of the C5-C6 segment. J Orthop Res $\underline{15}$, 342-347 
Cripton PA, BruehImann SB, Orr TE, Oxland TR, Nolte LP (2000): In vitro axial preload application during spine flexibility testing: Towards reduced apparatusrelated artifacts. J Biomech $\underline{33}, 1559-1568$

Deyo RA, Rainville J, Kent DL (1992): What can the history and physical examination tell us about low back pain? JAMA 268, 760-765

Dimova V, Lautenbacher S (2010): Chronischer Schmerz nach Operationen. Epidemiologie unter besonderer Berücksichtigung psychologischer Risikofaktoren. Anasthesiol Intensivmed Notfallmed Schmerzther $\underline{45}$, 488-493

Dreischarf M: zitiert nach mündlicher Mitteilung

Fanghänel J, Schulz F (1962): Mitteilung über eine Konservierungsflüssigkeit für anatomisches Präpariermaterial. Z Med Labortech $\underline{3}$, 329-32

Fanuele JC, Birkmeyer NJ, Abdu WA, Tosteson TD, Weinstein JN (2000): The impact of spinal problems on the health status of patients: have we underestimated the effect? Spine 25, 1509-1514

Freeman BJ, Davenport J (2006): Total disc replacement in the lumbar spine: a systematic review of the literature. Eur Spine J 15 Suppl 3, 439-447

Frick R, Leonhardt H, Starck D: Allgemeine Anatomie. Spezielle Anatomie I. Bd.1; Thieme, Stuttgart 1992

Goldstein H: Klassische Mechanik, 7. Auflage; Akademische Verlagsgesellschaft, Wiesbaden 1983

Gregersen GG, Lucas DB (1967): An in vivo study of axial rotation of the human thoraco-lumbar spine. J Bone Joint Surg Am 49, 247-254

Hart LG, Deyo RA, Cherkin DC (1995): Physician office visits for low back pain. Frequency, clinical evaluation, and treatment patterns from a U.S. national survey. Spine $\underline{20}, 11-9$

Hawellek T: Eine biomechanische Analyse des Lendenwirbelsegments L4/L5. Med. Diss. Göttingen 2008

Hering E, Martin R, Stohrer M: Physik für Ingenieure, 10. Auflage; Springer-Verlag, Berlin, Heidelberg 2007

Hilibrand AS, Carlson GD, Palumbo MA, Jones PK, Bohlman HH (1999):

Radiculopathy and myelopathy at segments adjacent to the site of a previous anterior cervical arthrodesis. J Bone Joint Surg Am 81, 519-528

Hubert J: Messungen zur räumlichen Kinematik des Lendenwirbelsegments L1/L2. Med. Diss. Göttingen 2009

Jason C, Fanuele JC, Nancy JO, Birkmeyer NJ, William A, Abdu WA, Tor D, Tosteson S, James $\mathbf{N}(2000)$ : The impact of spinal problems on the health status of patients: have we underestimated the effect? Spine 25, 1509-1514 
Junghanns H: Nomenclatura columnae vertebralis. (Die Wirbelsäule in Forschung und Praxis. Bd. 75); Hippokrates-Verlag, Stuttgart 1977

Kalscheuer A: Kinematik des Halswirbelsäulensegments C3/C4 unter systematischer Vorlastvariation. Med. Diss. Göttingen 2001

Klein P, Sommerfeld P: Biomechanik der Wirbelsäule: Grundlagen, Erkenntnisse und Fragestellungen. 1. Auflage; Urban und Fischer, München, Jena 2007

Kohlmann T, Schmidt CO (2005): Was wissen wir über das Symptom Rückenschmerz? Epidemiologische Ergebnisse zu Prävalenz, Inzidenz, Verlauf, Risikofaktoren. Z Orthop Ihre Grenzgeb 143, 292-298

Kubein-Meesenburg D, Nägerl H, Fanghänel J (1990): Elements of a general theory of joints. 1. Basic kinematic and static function of diarthrosis. Anat Anz 170, 301-308

Kubein-Meesenburg D, Nägerl H, Fanghänel J (1991): Elements of a general theory of joints. 4. Coupled joints as a simple gear system. Anat Anz 172, 309-321

Lang E, Eisele R, Kastner S, Liebig K, Martus P, Neundörfer B (2000): Ergebnisqualität der ambulanten Versorgung von Patienten mit chronischen Rückenschmerzen. Schmerz 14, 146-159

Mansour M: Die biomechanische Funktion der Articulationes zygappophysiales der Lendenwirbelsäule - eine 6-D Analyse der Bewegungsstrukturen des Segments L 3/4. Med. Diss. Göttingen 2001

Moll KJ, Moll M: Anatomie. 18. Auflage; Urban und Fischer, München, Jena 2006

Morris J, Watson PJ (2011): Investigating decisions to absent from work with low back pain: a study combining patient and GP factors. Eur J Pain 15, 278-85

Nägerl H : Biomechanische Prinzipien in Diarthrosen und Synarthrosen. Med. Habil.-Schr. Göttingen 1990

Nägerl H, Kubein-Meesenburg D, Fanghänel J (1990): Elements of a general theory of joints. 2. Introduction into a theory of synarthrosis. Ann Anat 171, 323-333

Nägerl H, Kubein-Meesenburg D, Fanghänel J (1992): Elements of a general theory of joints. 7. Mechanical structures of the relative motion of adjacent vertebrae. Ann Anat $\underline{174}, 66-75$

Nägerl H, Kubein-Meesenburg D, Cotta H, Fanghänel J, Kirsch S (1993a): Biomechanische Prinzipien in Diarthrosen und Synarthrosen. Teil II: Die Articulatio Humeri als Dimeres Kugelgelenk. Z Orthop 131, 293-301

Nägerl H, Kubein-Meesenburg D, Cotta H, Fanghänel J, Kirsch S (1993b): Biomechanische Prinzipien in Diarthrosen und Synarthrosen. Teil III: Die Mechanik des Tibiofemoralgelenks und die Rolle der Kreuzbänder. Z Orthop 131, 385-396 
Nägerl H, Kubein-Meesenburg D, Cotta H, Fanghänel J, Rossow A, Spiering S (1995): Biomechanische Prinzipien in Diarthrosen und Synarthrosen, Teil IV: Zur Mechanik der Wirbelsäule im Lendenbereich. Eine Pilotstudie. Z. Orthop. 133, 481-491

Nägerl H, Hawellek T, Lehmann A, Hubert J, Saptschak J, Dörner J, Raab B, Fanghänel J, Wachowski MM (2009): Non-linearity of flexion-extension charac teristics in spinal segments. Acta Bioeng Biomech 11, 3-8

Nielsen C F, Annertz M, Persson L, Wingstrand H , Saveland H, Brandt L (1997): Fusion or Stabilization Alone for Acute Distractive Flexion Injuries in the Mid to Lower Cervical Spine? Eur Spine J $\underline{6}, 197-202$

Niethard U (2011): Für eine konservative Orthopädie und Unfallchirurgie. Orthopade $4,317-31$

Panjabi MM, Summers DJ, Pelker RR, Videman T, Friedlaender GE, Southwick WO (1986): Three-dimensional load-displacement curves due to forces on the cervical spine. J Orthop Res $\underline{4}, 152-161$

Parnianpour M, Li F, Nordin M, Kahanovitz N (1989): A data base of isointerial trunc strength test against three resistance levels in sagittal frontal and transverse plancs in normal male subjects. Spine 14, 409-411

Ralston HJ (1961): Uses and limitations of electromyography in quantitative study of skeletal muscle function. Am J Orthod Dentofac $\underline{47}, 521-526$

RohImann A, Bergmann G, Graichen F, Weber U (2000): Changes in the Loads on an Internal Spinal Fixator after Iliac-Crest Autograft. J Bone Joint Surg [Br] $\underline{82}$, 445-449

Rousseau MA, Bradford D, Hadi T, Pedersen K, Lotz J (2006): The instant axis of rotation influences facet forces at L5/S1 during flexion/extension and lateral bending. Eur Spine J 15, 299-307

Schäfer W: Messung der räumlichen Mikrobewegung und der Verbiegung des Femurschaftes von Hüft-Endo-Prothesen in Abhängigkeit eines räumlichen Kraftsystems. Phys. Diplomarbeit (IV. Physikalisches Institut der Georg-AugustUniversität Göttingen) Göttingen 1995

Schendel MJ, Wood KB, Butterman GR, Lewis JL, Ogilvie JW (1993): Experimental measurement of ligament force, facet force and segment motion in the human lumbar spine. J Biomech 26, 427-438

Schmidt H: zitiert nach mündlicher Mitteilung

Schmidt H, Heuer F, Claes L, Wilke HJ (2007): The relation between the instantaneous center of rotation and facet joint forces $-A$ finite element analysis. Clin Biomech 23, 270-278 
Schmidt H, Heuer F, Wilke HJ (2009): Which axial and bending stiffnesses of posterior implants are required to design a flexible lumbar stabilization system? J Biomech $\underline{42}, 48-54$

Schünke M, Schulte E, Schumacher U: Prometheus. LernAtlas der Anatomie. Allgemeine Anatomie und Bewegungssystem. 1. Auflage; Thieme Verlag, Stuttgart 2005

Silver PHS (1954): Direct observation of changes in tension in the supraspinous and interspinous ligaments during flexion and extension of the vertebral column in man. J Anat $\underline{88}, 550-551$

Spiering S: Kinematik der Lendenwirbelsäule unter Lastvariation. Phys. Diplomarbeit (IV. Physikalisches Institut der Georg-August-Universität Göttingen) Göttingen 1995

Spiering S: Drei Methoden zur Evaluation der mechanischen Funktion von Synarthrosen und Diarthrosen. Med. Diss. Greifswald 2002

Steffen R, Rubin RK, Baramki HG, Antoniou J, Marchesi D, Aebi MA (1997): A new technique for measuring lumbar segmental motion in vivo. Spine $\underline{22}$, $156-166$

Teichmann H: Physikalische Anwendungen der Vektor- und Tensorrechnung. Bd.1; BI-Verlag, Mannheim, Wien, Zürich 1973

Wachowski MM, Ackenhausen A, Dumont C, Fanghänel J, Kubein-Meesenburg D, Nägerl H (2007): Mechanical properties of cervical motion segments. Archive of Mechanical Engineering 54, 5-15

Wachowski MM, Mansour M, Lee C, Ackenhausen A, Spiering S, Fanghänel J, Dumont C, Kubein-Meesenburg D, Nägerl H (2009): How do spinal Segments move? J Biomech 42, 2286-2293

Wachowski MM, Mansour M, Hawallek T, Kubein-Meesenburg D, Hubert J, Nägerl H (2010a): Parametric Control of the Stiffness of Lumbar Segments. Strain DOI: $10.1111 /$ j.1475-1305.2009.00686.x

Wachowski MM, , Hawellek T, Hubert J, Lehmann A, Mansour M, Dumont C, Dörner J, Raab B, Kubein-Meesenburg D, Nägerl H (2010b): Migration of the instantaneous axis of motion during axial rotation in lumbar segments and role of the zygapophysial joints. Acta Bioeng Biomech 12, 39-47

Waldeyer A, Mayet A: Anatomie des Menschen I. 16. Auflage. Walter de Gruyter Berlin, New York 1993

Watson PJ, Main CJ, Waddel G (1998): Medically certified work loss, recurrence and costs of wage compensation for back pain: a follow up study of the working population of Jersey. Br J Rheumatol $\underline{37}$, 82-86 
Wenig CM, Schmidt CO, Kohlmann T, Schweikert B (2009): Costs of back pain in Germany. Eur J Pain 13, 280-286

Wetz HH, Jakob HAC (2001): Die Bedeutung des dreidimensionalen Bewegungsablaufs des Femurotibialgelenks für die Ausrichtung von Knieführungsorthesen. Orthopade $\underline{30}, 196-207$

White A, Panjabi M: Clinical Biomechanics of the Spine. 2. Auflage. J.B. Lippincott Company, Philadelphia 1990

Wilke HJ: Experimentelle Untersuchung zur Biomechanik der Wirbelsäule und ihrer Stabilisierung. Humanbiologische Diss. Ulm 1993

Wilke HJ, Krischak S, Lutz EC (1996): Formalin fixation strongly influences biomechanical properties of the spine. J Biomech. $\underline{29}, 1629-1631$

Wilke HJ, Kettler A, Hartwig E, Schultheiß M, Claes L (2002): Mechanically simulated muscle forces strongly stabilize intact and upper cervical spine specimens. J Biomech $\underline{35}, 339-346$

Yao W, X Mai, Luo C, Ai F, Chen Q (2011): A cross-sectional Survey of Nonspecific Low Back Pain Among 2083 Schoolchildren in China. Spine 36, 1885-1890

\section{Zhu Q, Larson CR, Sjovold SG, Rosler DM, Keynan O, Wilson DR, Cripton PA,} Oxland TR (2007): Biomechanical evaluation of the Total Facet Arthroplasty System: 3-dimensional kinematics. Spine $\underline{32}, 55-62$ 